\title{
ANÁLISE NUMÉRICA DO AQUECIMENTO DE PLASMA, GERAÇÃO DE CORRENTE E FLUXO POR ONDAS DE ALFVÉN NO TOKAMAK TCABR
}

\section{Gesil Sampaio Amarante Segundo}
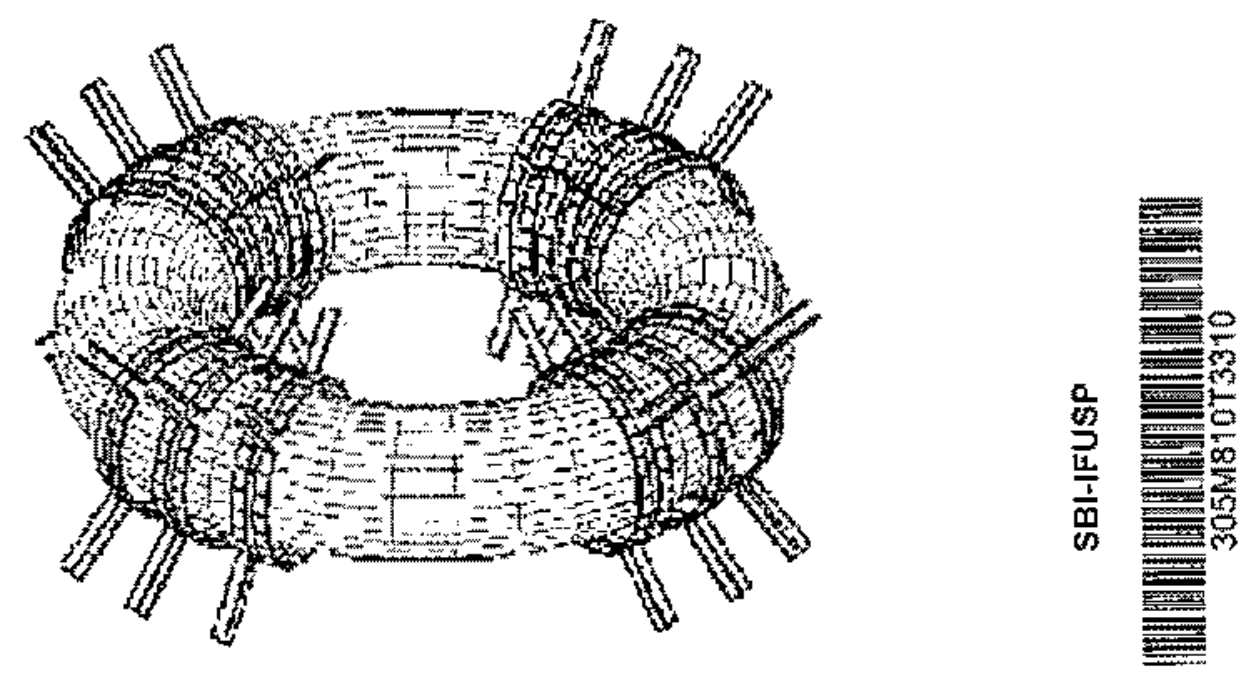

Banca Examinadora:

Prof. Dr. Artour Elfimov - Orientador (I.F, U.S.P.)

Prof. Dr. Ibere Luiz Caldas (I.F.U.S.P.)

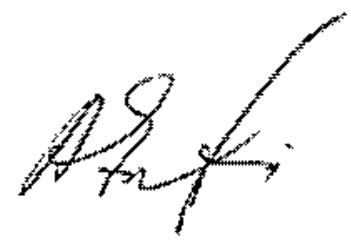

Prof. Dr. Josif Frenkel (I.F.-U.S.P.)

Prof. Dr.Paulo Hiroshi Sakanaka (1.E.-UniCamp)

Prof ${ }^{n}$. Dra ${ }^{a}$ Maria Virgínia Alves (IN.P,E.)

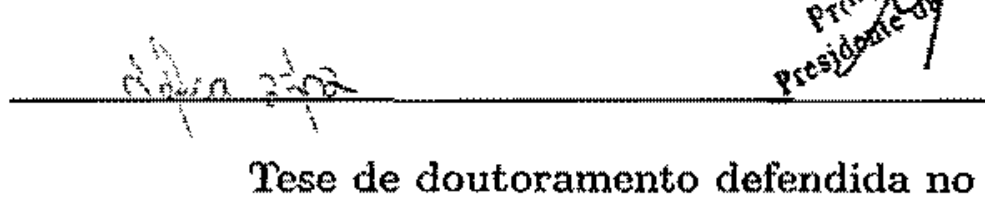

Instituto de Física da Universidade de São Paulo

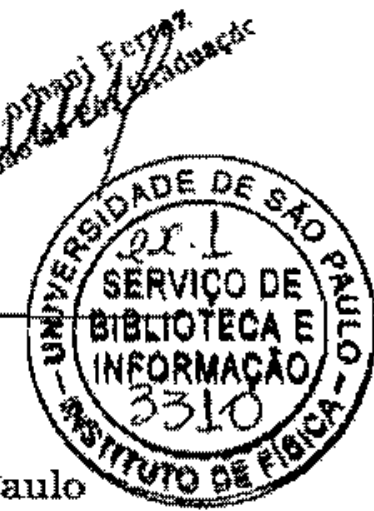

Săo Paulo, 23 de março de 1999. 


\subsection{4$$
\text { A } 4850
$$

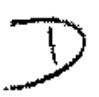 \\ $e x \cdot 1$}

FICHA CATALOGRÁFICA

Preparada pelo Serviço de Bibliạteca e Informação do Instituto de Física da Universidade de São Paulo

Amarante Segundo, Gesil Sampaio

Análise Numérica do Aquecimento de Plasma, Geraçăo de Corrente e Fluxo por Ondas de Alfvén no Tokamak TCABR. Săo Paulo, 2000.

Tese (Doutoramento) - Universidade de São Paulo. Instituto de Fisica - Departamento de Fisica Aplicada

Orientador: Prof. Dr. Attour Elfimov

Area de Concentração: Física de Plasmas

Unitermos: 1. Plasmas; 2. Ondas de Alfvèn;

3. Fusāo Termonuclear Controlada;

4. Geraçăo de Correntes e Fluxos em Plasmas;

5. Barreiras de Transporte.

USP/IF/SBI-000/2000 



\section{Agradecimentos}

Quero expressar meus mais sinceros agradecimentos às pessoas que me acompanharam de uma forma ou de outra ra jornada destes ultimos anos:

Aos meus orientadores, os Professores Artour Elfimov e Ricardo Galvão, pela sempre presente preocupacäo com minha formaçăg, presteza, amizade e, principalmente pela paciência comrigo.

Aos Professores David Ross e Swadesh Maluajan (Universidade do Texas em Austin) pela grande ajuda com o código toroidal e discussôs, e assimn como todos no Fusion Research Center, a acolhida amistosa nos dois meses em que le estive. Especialmente o amigo Romik Chatterjee.

Aos colegas do Laboratorio de Fisica de Plasmas da USP, Ablicio Reis, Aluisio Fagundes, Alvaro Vannucci, André Alves Ferreira, Ande Twzsel, Anselmo Rodrigues, Angela Pizzo, Edson Ozono, Edson (Banzai) Sanada, Elion da Silva, Erich Olschevski, Ernesto Lerche, Fabio Pallatino, Francisco Tadeu Degasperi, Josẻ Hélder Severo, Iberê Caldas, Ibrahim EI Chamaa Neto, Ing Tan, Ivan dos Santos, Iyan Nascimento, Ivan Jancov, Juan Elisondo, Kai Ulmann, Kênya de Oliveira, Leonid Ruchko, Maria Vittória Aeller, Mauro Sérgio de Araújo,

Murilo Baptista, Mutsuko Kucinski, Nélio Nunes, Renata Favalli, Roberto Bianchini, Ruy Pepe da Silwa, Sílvio Luiz de Souza, Valdemar Belintani Jr, Victor Mammana, Vladimir Tsypin, Wanderley Pires de Sá, Yuri Kusnetsov pelo respeito, comparheirismo, ajuda e amizade.

Ass secretárias, Eleonora Lo Duca, Maria Mavilla Vara, pela valorosa colaboraçäo.

A FAPESP pelo indispensável auxílio financeiro.

Aog amigos do peito Adriana Marques, Agostinho Serrano Neto, Cristina Collares Niro, Elisângela Manfra José Antonio Sevidanes da Matta, Nestor Cortez Satavedra, Roberto Niro e Zeide Guvaloanti

A uma amiga muito especial, Elisabete Gentil. 

Aos meus irmäos, Márcia Goretti Amarante Marcus Vinícius de Jesus Amarante por tudo o que irmăos podem ser de bom. Junto a eles, gostaria de inchir a Senhora Maria Elieci de Jesus Santos pela ajuda, torcida o oraços.

A minha mäe, Dilma Maria da Concetçăo, sempre um modelo para mím.

A Luciane Aparecida Goulart, por ter me dado o maior presente de todos, a minha Cllha, e por dela cuidar tāo ben.

A minha flha, Beatriz Goulart Amarante porque desde 08/12/97, dia em que nasceu, me hez mais completo, e feliz. 


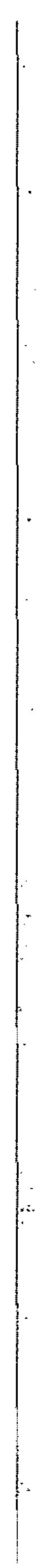




\section{Resumo}

Este trabalko tem como objetivo a determinaçäo dos melhores regimes e parâmetros para as experiencias de aquecimento e geraçăo de corrente e fluxo no tokamak TCABR. Grande parte do trabalho dispensado no sentido da escolha dos melhores modos e freqüências para operação das antenas do TCABR está presente nesta tese.

Em suscinto resurno, no segundo capítulo é apresentada a moderna teoria de ondas de Alfvén. No início, è apresentada uma introduçāo bâsica até a derivaçāo do tensor dielétrico com termos cinéticos em primeira ordem. A relação de dispersão para plasmas homogêneọs é demonstrada, a fim de servir de introdução à seçăo seguinte que mostra o efeito de conversão de modos. Cálculos com geometria cilindrica do aquecimento e o efeito de impurezas minoritárias no plasma seguem com a demonstração da presença dos modos globais de Alfvên.

No terceiro capítulo é introduzido o código bidimensional. Este código foi trazido da Universidade do Texas e adaptado aos parămetros do TCBR. As principais modificaçōes provocadas pelos efaitos da configuraçăo toroidal são mostradas e analisadas.

No quarto capitulo, a teoria e os resultados principais dos cálculos das forças ponderomotoras sẵo apresentadis. Também é estudado o efeito da rotaçã̃o poloidal provocada pelas ondas de Alfyén no transporte neoclássico.

O quinto e viltimo capitulo apresenta uma sintese dos resultados obtidos ao longo desta dissertaç̧ão. 



\section{Abstract}

This work aims the determination of the best regines and parameters for the heating, current drive and flow generation experiements in the tokamak TCABR. The majority of the work done to choose the best modes and frequencies for operation of the antennas of TCABR is present in this thesis.

In the second chapter the modern theory of Alver waves is presented. In the beginning, a basic introduction is presented until the derivation of the dielectric tensor with kinetic terms in first order. The dispersion relation for homogeneous plasmas is demonstrated, in order to introduce the following section which shows the mode conversion effect. Calculations with cylindrical geometry of the heating and the effect of minority ions in the plasma proceed with the dernonstration of the presence of the Global Alfvén Waves (GAW).

In the third chapter the twodimensional code is introduced. This code was brought: from the University of Texas at Austin and adapted for calculations with the parameters of TCBR. The main effects of the toroidal model are analyzed.

In the fourth chapter, the theory and the principal results of the calculations of the ponderomotive forces are presented. The effect of the poloidal rotation induced by the Alfuen Waves on neoclassical transport is also studied.

The fifth and last chapter presents a synthesis of the results obtained along this dism Eertation. 


\section{Conteúdo}

1 INTRODUÇÄO

1.1 Fusăo Termonuclear Controlada $\ldots \ldots \ldots \ldots$

1.2 Sistemas Auxiliares de Aquecimento, Geração de Corrente e Fluxo . . . . 10

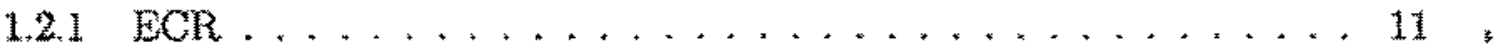

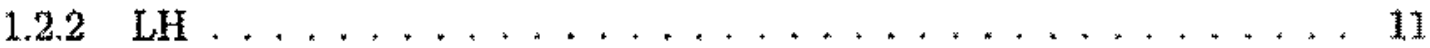

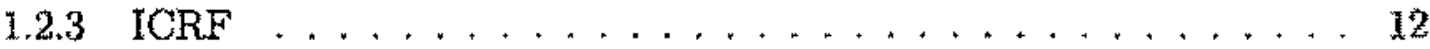

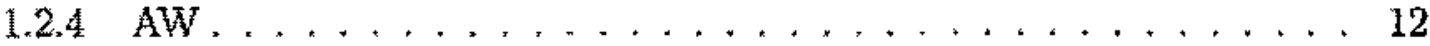

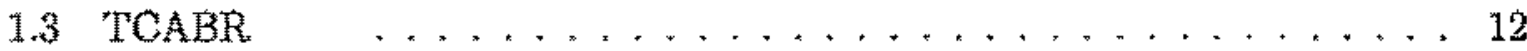

1.4 Proposta e Estrutura da Tese $\ldots \ldots \ldots \ldots$

2 TEORIA 18

2.1 Ondas de Alfvén - Introduçäo $\ldots \ldots \ldots \ldots$

2 Equaçoes Básicas, Tensor Dielétrico $\ldots \ldots \ldots \ldots$

2.2 .1 Função Distribuiç̃̃o no Espaço de Velocidades . . . . . . . 26

2.3 Relação de Dispersäo para Ondas de Alfvên (Plasma Homogêneo) . . . . 29

2.4 Ondas de Alfvën em Plasmas Cilíndricos lnomogêneos e Efeito de Conversāo de Modos $\quad \ldots \ldots \ldots \ldots \ldots \ldots$

2.4.1 Efeito te Conversāo de Modos $\ldots \ldots \ldots \ldots$. . . . . .

2.5 Análise do Efeito das Impurezas $\ldots \ldots \ldots \ldots \ldots \ldots$

2.5.1 Modelo Cilíndrico de Plasma e Resultados dos Cáloulos . . . . . 42

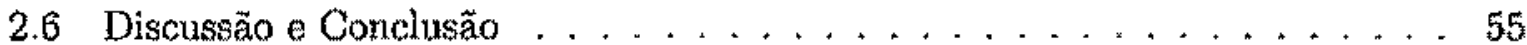

3 CÓDIGO TOROMAL

3.1 Histórico . . . . . . . . . . . . . . . . 50

3.2 Introduçà̃ $\ldots \ldots \ldots \ldots \ldots \ldots \ldots \ldots$ 


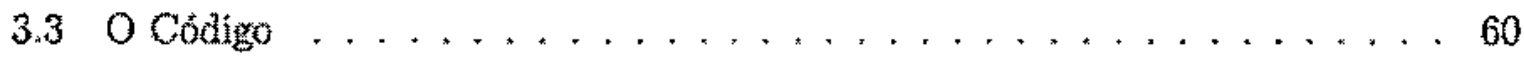

3.4 Dissipação da onda de Alfvén $\ldots \ldots \ldots \ldots \ldots \ldots \ldots$. . . . . .

3.5 Efeito de impurezas na dissipaçäo da onda de Alfvén $\ldots \ldots \ldots \ldots 69$

4 FORCAS PONDEROMOTORAS

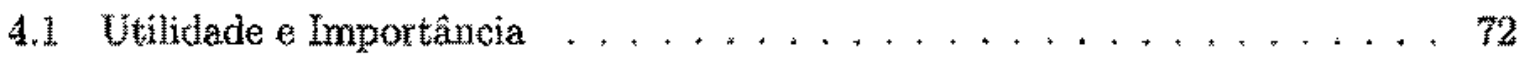

4.2 Equações Básicas para Forças e Fluxo de Plasma . . . . . . . . 75

4.2 .1 Forças Viscosas . . . . . . . . . . . . . . . . 75

4.2 .2 Forças Ponderomotoras . . . . . . . . . . . . . 76

4.3 Efeito da Rotaçào Cisalhada no Trarsporte Neoclássico . . . . . . . . 82

4.3.1 Equaçōes Diferenciais Para a Viscosidade Ionica . . . . . 83

4.4 Conclusões. . . . . . . . . . . . . . . . . . 87

5 SUMÁRIO E SUGESTÕES

5.1 Resumo dos resultados $\ldots \ldots \ldots \ldots \ldots \ldots \ldots \ldots . . \ldots$

5.2 Sugestōes para continuidade $\ldots \ldots \ldots \ldots \ldots \ldots \ldots$

A Derivação de coeficientes do tensor dielétrico em coordenadas cilíndricas (exemplo) 95

B Tensor dielétrico 105

C Solução da Equação para o Campo Elétrico 111

D Aplicando o Teorema de Poyting Complexo 116

E Construçāo do código toroidal 118

E.1 Resumo . . . . . . . . . . . . . . . . . . 118

E.1.1 Equação da onda . . . . . . . . . . . . . . . . 121 
E.1.2 Thansformada de Fourier $\ldots \ldots \ldots, \ldots \ldots 2$

E.1.3 Conservaçäo de Energia $\ldots \ldots \ldots \ldots$

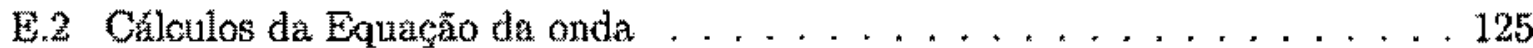

E.2.1 Componente radial $\ldots \ldots \ldots \ldots \ldots$

E.2.2 Componente perpendiculer . . . . . . . . . . 120

E.2.3 Eliminando $E_{\|} \ldots \ldots \ldots \ldots \ldots, \ldots \ldots$

F Forças ponderomotoras 140

F.0.4 Forças Viscosas Poloidais . . . . . . . . . . . . 143

G Efeito da rotação cisalhada nos modos Kink externos $\quad 147$ 
Os tokamaks sẫo sistemas toroidatis de confinamento magnético que utilizam-ge de uma corrente de equilibrio, criada na direçăo toroidal, como sistema principal de aquecimento, devido so efeito Joule. O problema que aparece é que a resistividade do plasma proporcional a $T^{-3 / 2}$ o aquecimento bhmico satura (em pouco menos que $2 \mathrm{KeV}$ ) antes de se obter a temperatura múnima requerida para a fusāo (por volua de $10 \mathrm{KeV}$ ). Por isso săo empregados sistemas auxiliares de aquecimento, mostrados en resumo na próxima secăo. Na Fig. 1, é mostrado um desenho do tokamak JET (Joint European Torus), o maior em funcionamento hoje.

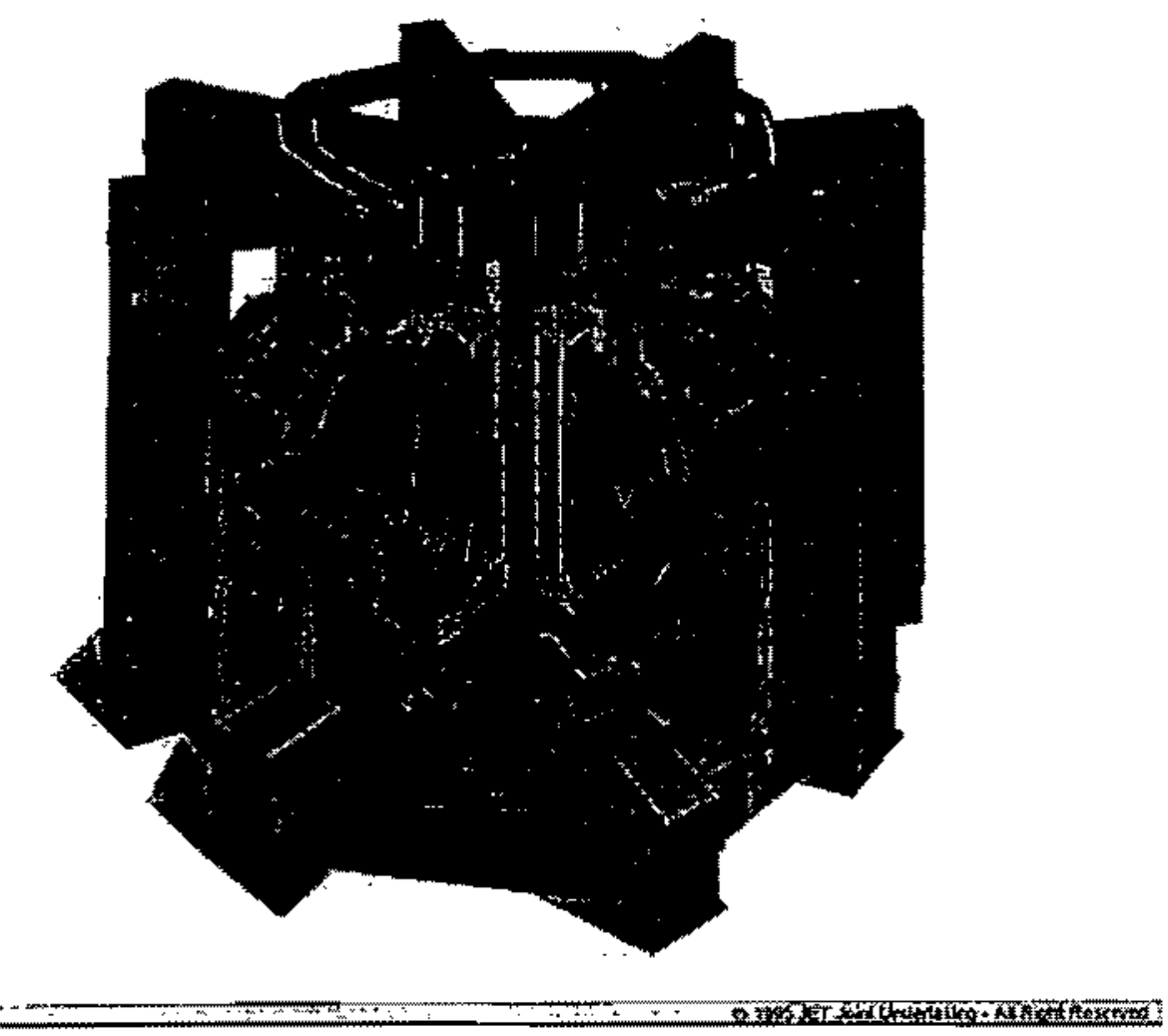

Fig. 1.1 - Desenho esquernático do tokanak JET, en funcionanento na Inglaterta

Pode-se perceber no desenho acima a câmara toroldal, as espiras responsấveis pelo campo magnético toroidal e transformadores. Todo o sistema funciona como urn enorme transformador, que induz uma corrente toroidal $j_{i}$, que por sua vez cria um campo magnético $B_{\theta}$ criando um efeito de compressão do plasma (efeito pinch), responsável pelo confinamento do plasma. O campo magnético toroidal intenso provocado pelas espi- 
ras em torno da câmara toroidal, associado com o campo poloidal (gerado pela corrente toroidal), cria uma configuraçầo helicoidal de linhas de força ao longo da câmara. Como B. nüo 6 constante na direcăo radial, esta disposicăo helicoidal näo é uniforme, formando superficies magnéticas sucessivas, cada uma com linhas de forga com helicidade diferente, e encerrando as superficies mais internas.

Abaixo, num esquema simples da geometria da camara toroidal de um tokanak como o JET (com seção toroidal em forma de D), pode-se vizualizar as diferentes auperficies magnéticas (definidas pela coordenada $\psi$, função de $\tau^{*}$ e $\theta$ ), também definidas como superfícies onde a pressäo cinética do plasma é constante (ver Ref. [2], Capítulo 12).

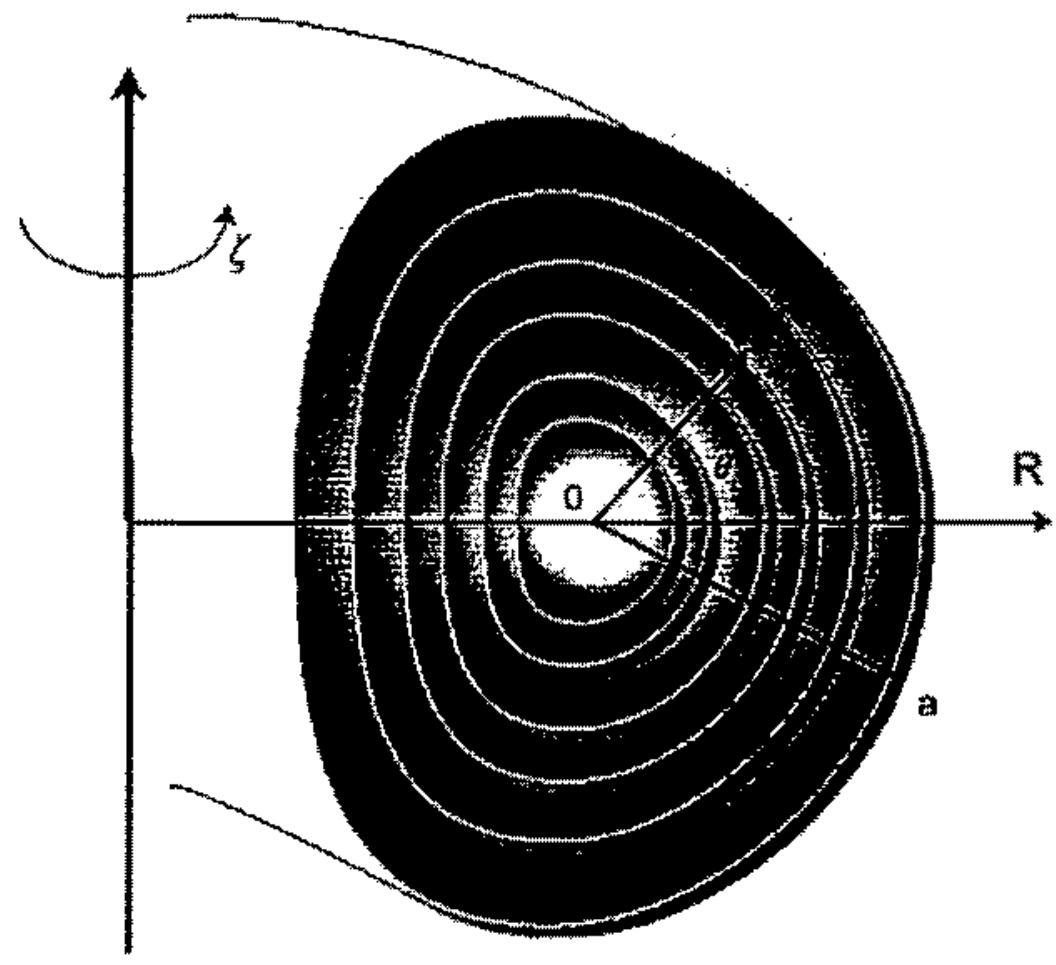

Figura 1.2 - Desento das superficies magneticas $\psi(f(r, \theta)$ num tokambk de forma alongada (D-shape).

O sistema pode ser mapeado usando-se diferentes sistemas de coordenadas. A partir do centro geométrico do torólde, pode-se definir um ponto pela sua distancia $(Z)$ ao eixo equatorial, pelo ângulo toroidal (6) e pela distância to elxo principal $(R)$. De outra 
maneira, supondo-se perfeita simetria toroidal, a posição de qualquer porção do plasma pode ser determinada pela superfície de fuxo magnético, $b(r, \theta)$ e pelo angulo $\theta$ fonde $\psi=0$ determina o eixo magnêtio da coluna de plasma, onde a pressão cinética é máxima) e o limite da coluan de plasma é determinado pela condição $p=0($ th $=a)$. Esta disposiçăo em forma de $D$ é usada nos tokamaks maiores para suprimir o efeito de determinadas instabilidades.

Para entender melhor as superficies magnéticas (ou superficies de fuxo maznético), é importante saber que o campo magnético gerado pelas espiras, ná direçăo toroidal, ê muito maior que a componente poloidal gerada pela corrente toroidal de plasma. O primeiro varia com $1 / R$, sendo que, para configuraçoes em que $a<<R$, pode ser considerado praticamente unifome. 0 segundo cresce com a distância ao eixo magnetico da coluna de plasma, o que resulta numa disposiçäo helicoidal do campo magnético resultante ao longo da colnma toroidal. A direção do campo magnético é entăo variada para cada $\tau$ e $\theta$, sendo possivel descrever superfícies concêntricas ao longo de um corte transversal, sendo que cada superficie apresenta as linhas de campo resultantes paralelas. Este cisalhamento magnético e muito importante, entre outras coisas porque atua no sentido de dificultar a migraçäo das partículas do plasma entre as superficies de fluxo (pelo efeito de "congelamento" das linhas de campo magnetico. As partículas tendem a seguir ao longo da linha de campo a migração de uma linha para outra, neste caso, também resulta numa mudanģa de direção do movimento). Uma quantidade que descreve o cisalhamento magnético para cada superfície magnética é a chamada transformada rotacional inversa:

$$
q(\psi)=\frac{r B_{\xi}}{R B_{0}}
$$

que é definida como a razão entre o número de voltas que ass linhas de campo realizam no sentílo toroidal e o número de voltas que realizam no sentido poloidal fo valor da transformata inversa, na borda da coluna de plasma, é chamado de "fator de segurança"). 
Hă, todavia, configuraçôes mais simples, onde a seção transversal da coluna de plasma é circular e pode-se aproximar $\psi(r, \theta) \rightarrow \psi(r) \rightarrow r$. As coordenadas neste sistema sã̃o $\zeta, \theta, r$ e esté é o caso do TCABR.

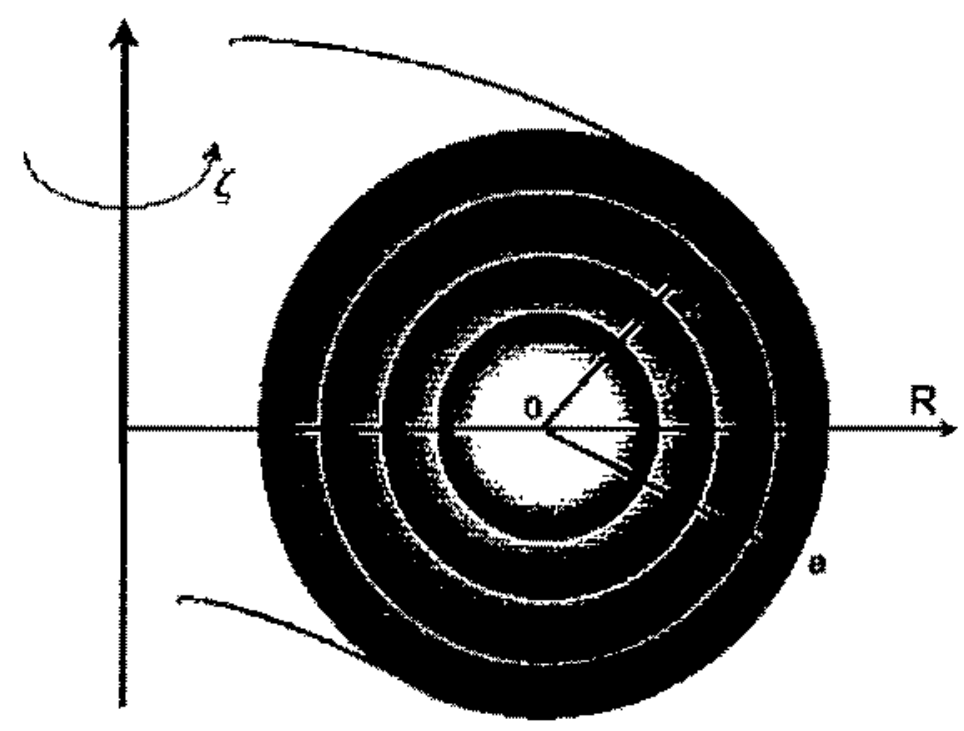

Figura 1.3 - Superficies de campo magnético para um tokamak de coluna circular.

Em certas circunstâncias, pode-se simplificar ainda mais o modelo a ser estudado; se os efeitos da curvatura toroidal a da variação do campo magnético em $R_{3}$

$$
B_{z}=B_{0} / R
$$

forem pequenos, a geometria do problema se reduz a uma configuração cilíndrica periódica (Fig. 1.4).

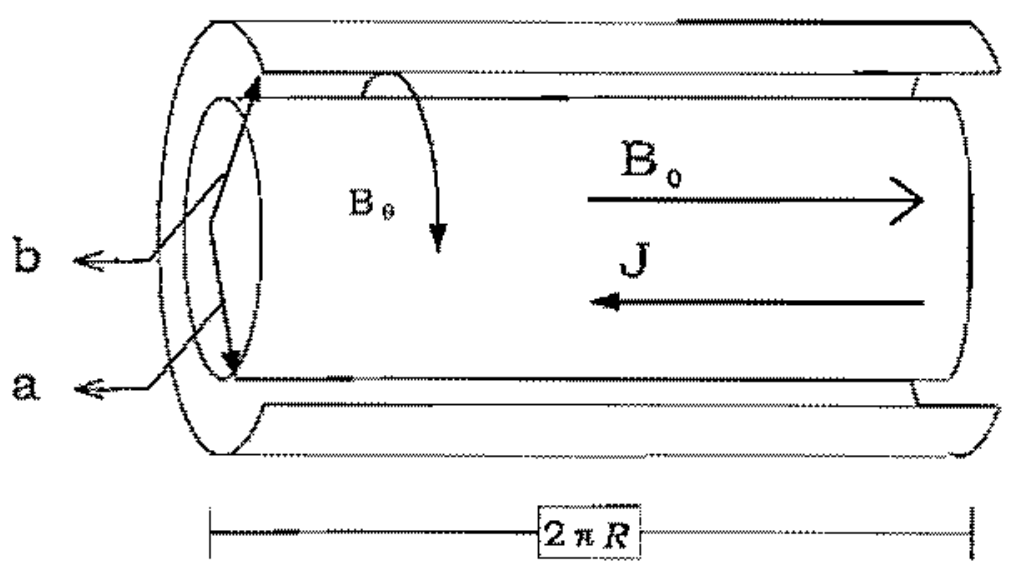

Fig. 1.4 - Aproximaçẵo cilindrica. 


\subsection{Sistemas Auxiliares de Aquecimento, Geraçāo de Corrente e Fluxo}

Basicarnente, as duas propostas principais de aquecimento auxiliar de plasmas em tokamaks stăo:

- Injeção de partículas neutras (NBI) - Injeção tangencial de partículas neutras de alta energia que são ionizadas e termalizam, aquecendo o plasma.

- Aquecimento por ondas - Ondas eletromagnéticas de vários típos dependendo de que ressonância se queira excitar no plasma, sāo lançades por antenas ou guias de onda, especialmente projetados para oferecerem o maior efeito de acoplamento possível e transformarem a energia da onda em energia cinética de porçōes específicas das particulas. Efeitos secundários seriam responsávets pela clifusão desta energia por toda a coluna de plasma.

Outro efeito que se tenta obter com os métodos descritos acima é a geraçāo de correntes e fluxos no plasma. O principal objetivo aqui ẻo o de criar um reggime estacionário para a configuraçăo do tokamak, através da criaçâo de uma corrente suplementar na direção toroidal, diferente da corrente toroidal pulsada produxida pelos transformadores, de forma a evitar o ciclo de aquecimento e resfriamento que destrój a primeira parede de contençầo do plaşma.

Outro objetivo é o de criar barreiras de transporte. Efeitos diversos, ainda não completamente entendidos, levam a uma perda da estabilidade da configuração magnética do plasma e terminam por provocar transportes radiais anômalos, com perdas significativas de particulas na direçâo das paredes metálicas. A esta perda de plasma soman-se as partículas presentes na parede metálica que säo então arremessadas para o centro do plasma e aumentam mais ainda a perda de energia do sistema.

Recentemente, foi demonstrado que uma rotação poloidal. cisalhada na coluna de 
plasma suprime a turbulència na sua periferia (ver Refs. [3], [4],[5]) e nos experimentos em que ocorre a transiçäo L-H' uma forte rotaçäo cisalhada na periferia da coluna de plasma está sempre presente. Tambêm, mais reentemente, foram detectadas barreiras Internas de transporte (ITB) ${ }^{6]}$. Tais barreiras se situam não na periferia, mas na regrăo intermediária do raio do tokamak, e aumentam ainda mais a estabilidade do plasma. A geração destas barreiras de transporte é mais um objetivo buscado ațravés dcs mesmos métodos auxiliares de aquecimento.

Entre as várias taixas de frequiênela disponíveis para os tokamaks, com campos magnéticos entre 0,5 $5 T$, pode-se citar, resumidamente, os seguintes:

\subsubsection{ECR}

Electron Cyclotron Resssonance - Ressonância ciclotrônica eletrọniç $-\omega_{c y}=e B / m_{e} c$ - Lançado por guias de onda. Freqüuencias na faixa de 60 a $150 G H z$. Tem as vantagens de ter sido testado experimentalmente ter uma teoria simples. Como desvantagens, apresenta um alto custo, especialmente dos gyrotrous utilizados para o carregamento das antenas, fraca geração de corrente e diminuiçzono rápida da eficiencia de acoplamento com o aumento da densidade do plasma.

\section{$1.2 .2 \mathrm{LH}$}

Low-Hybrid - Ressonancia hibrida inferior $+\omega_{t h} \approx \sqrt{\omega_{c \varepsilon} \cdot \omega_{\text {flt }}}-$ Lançado por guias de onda - Frequêtncias na faixa de $100 M H z$ z $2,5 G H z$. Corro vantagem prinoipal apresenta aquecimento e geração de corrente experimentalmente demonstrados; mas apresenta um limite para densidades de $3 \times 10^{13} \mathrm{~cm}^{-3}$, acima da qual diminui tambêm substancialmente a eficiência.

\footnotetext{
${ }^{1}$ Loxswidgh Confinement. Este rnodo de alto confinamento é um regime experimentalmente verificado en grandes tokumaks, nos quais o tampo de confunamento do plasmat praticamete dobra.
} 


\subsubsection{ICRF}

Ion Cyclotron Range of Frequencies - $\omega_{\mathrm{c} i} \simeq Z e B / m_{i} c$ - Lançado por antenas Freqüências na faixa de 5 a $50 \mathrm{MHz}$. Tem eficácia comprovada para aquecimento de elétrons e íons. Permite operação em pulsos longos e o aparato de radiofreqüência é simples. Como desvantagens necessita de experiências de geração de corrente e requer controle da densidade e boronizaçāo, por causa da geraçāo de impurezas.

\subsubsection{AW}

Ondas de Alfvén - $\omega_{\Lambda}=B / \sqrt{4 \pi m_{i} n_{i}}$ - Lançado por antenas helicoidais ou de multiestruturas - Freqüências na faixa de 1 a $5 \mathrm{MHz}$. Tem comprovado experimentalmente o aquecimento dos elétrons, os geradores são baratos e os dispositivos de RF são simples. Como desvantagens, carece de experiências de geraçāo de corrente e requer controle da densidade e boronização, por causa da geração de impurezas.

No capítulo 2 retornaremos ao tema da utilização de ondas para aquecimento do plasma, com mais detalhes e focada nas ondas de Alfvén, principal objeto de estudo do tokamak TCABR em operação no Instituto de Física da Universidade de São Paulo.

\subsection{TCABR}

Trazido de Lauzanne, Suíça, onde chamava-se TCA (Tokamak Chauffage Alfvén), após um longo caminho e processo de reconstrução, que começou com seu embarque em 94 e culminou com sua inauguração em outubro de 1999, o TCABR tem como principal tarefa continuar os estudos de aquecimento e iniciar experimentos com geração de corrente e fluxos no plasma através de ondas de Alfvén, para a obtenção de barreiras de transporte e do modo de alto confinamento (Modo H). Será dada, também, continuidade aos estudos 
de disrupşôs e turbulência em tokamaks que já vinham sendo realizados no TBR-1. No momento em que este texto esta sendo escrito, está sendo feita a instalaçăo do sistema de antenas do TCABR (Fig. $1.5-1.7$ ).

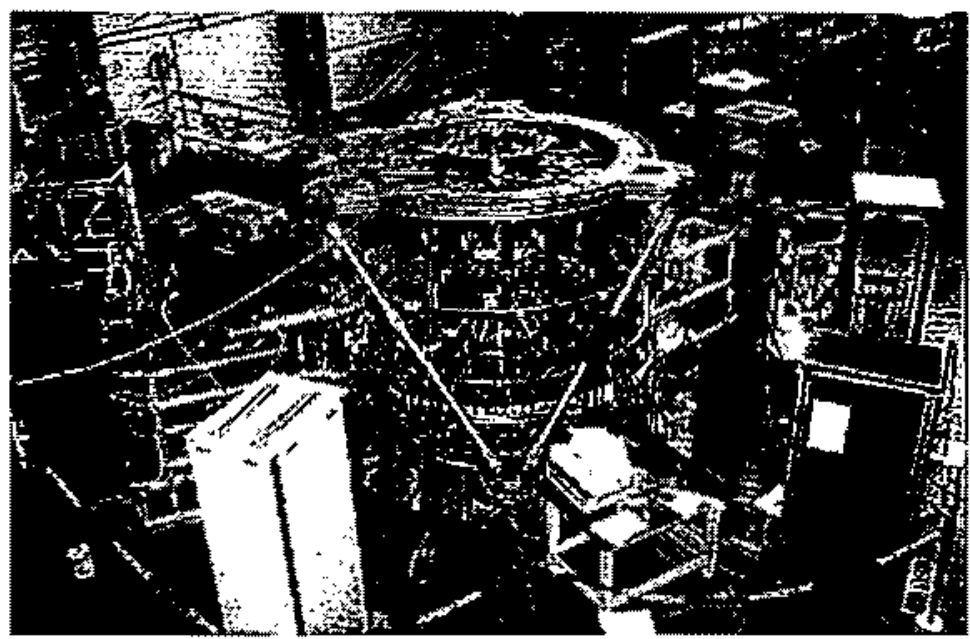

Figura 1.5 - Vista do TCABR.

Este sistema de antenas representa a principal mudança com relaçäo à antiga montagem em Lauzanne. $O$ antigo arranjo experimental näo permitia que se excitassem modos bem determinados, que levava à geração de modos superficiais indesejăveis e conseqüente aumento do transporte e de impurezas que irradiam fortemente, retirando energia do plasma.

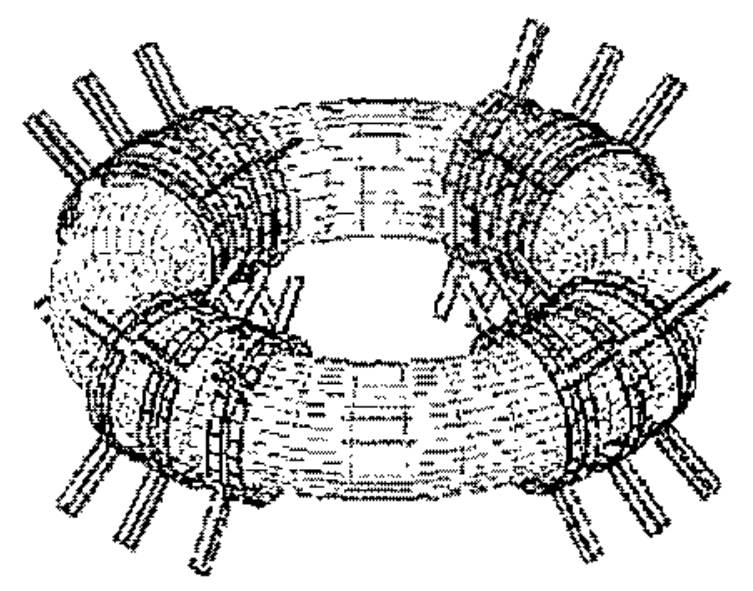

Figura 1.0 . Esquema da colocaţăo dos elementes da antena do TCABR. 


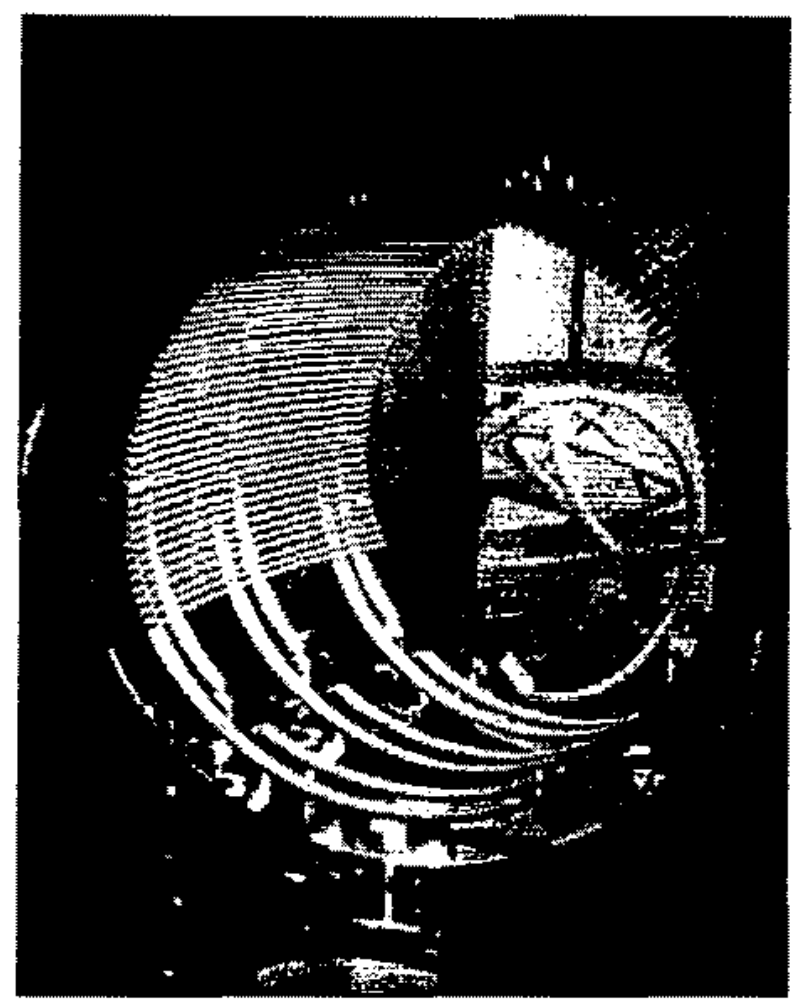

Figura 1.7. Parte do sistema de entenas do TCABR.

A seguir, uma tabela dos dados do TCABR:

\begin{tabular}{|cc|}
\hline Raio Maior $(R)$ & $61 \mathrm{~cm}$ \\
Raio Menor $(a)$ & $18 \mathrm{~cm}$ \\
Raio da Antena $\left(R_{k}\right)$ & $18,4 \mathrm{~cm}$ \\
Raio da Parede Metalica $\left(R_{w i}\right)$ & $23 \mathrm{~cm}$ \\
Corrente de Plasma $\left(I_{p}\right)$ & $80 \mathrm{kA}$ \\
\hline
\end{tabular}

\section{Sistemas de Diagnósticos:}

Os diagnósticos que se encontram em funcionamento são os seguntes:

Diagnósticos eletromegnéticos: bobinas de Rogowski para as medidas de corrente de plasma, corrente nas bobinas do campo toroidal, campo vertical/horizontal e nas bobinas de aquecimento thmico. Um sinal de voltagem para medida da corrente nas bobinas toroidais é obtido, tambérn, através de cueda de voltagem em uma resistência calibrada, 
em sếrie com as bobinas toroidais. Voltagem de enlace, obtida através de 8 espiras independentes, instaladas ao rexlor da câmara de vácuo, do lado de fora, sendo 4 no lado interno e 4 no lado externo. Bobinas cosseno e seno para a medida das posições horizontal e vertucal da coluna de plasma. Bobinas de Mirnov para a deteç̧ăo de oscilaçōes MHD.

Espectrômetro óptico para a medida de impurezas, temperatura ionica, densidade, temperatura eletrônica e $Z_{\text {téction }}$

Sonda eletrostática múltipla (4 alehrodos) com varredura radial rápida automatizada com tempo de varredura de $10 \mathrm{~ms}$ e excursăo de $8 \mathrm{~cm}$, permitindo avangar $3 \mathrm{~cm}$ para dentro do plasma, possibilitando obter-se 4 varreduras por descarga.

Detector de $H_{\alpha}$ park a medida de intensidade da linha $H_{a}$ para monitorar processos radiativos na periferia do plasma.

Bobina diamagnética para a medida do beta poloidal e balanço de energia em combinação com as medidas de bolometria

Em fase de teste encontram-se os seguintes diagnósticos:

Interferometro de microondas de $150 \mathrm{GHz}$, capaz de medir a densidade ern 7 posiç̧es radiais diferentes e composto de 3 canais independentes.

Bolômetro para a mediçăo da radiação emitida pelo plasma.

Bobina de fluxo (saddle coil e pick-up coils) para a medida da posiçăo da coluna de plasma e superfície de fluxo limite do plasma.

Em fase final de preparaçăo encontram-se:

- Analisador de Partículas Neutras para medida da temperatura de ions e densidade de partículas neutras.

Diagnóticos em fase de planejamento e solicitação de recursos:

- Espalhamento Thomson - para medidas de temperatura e densidade de elétrons com alta resolução temporal espacial. 
- Reffectômetro de xuicroondas para medidas de oscilações de densidade e de densidade de elétrons.

- Radiômetro por emissāo eletrociclotrônica para a medida do perfil radial da temperatura de elétrons.

\subsection{Proposta e Estrutura da Tese}

Este trabalho tem como objetivo a determinaçāo dos melhores regimes e parâmetros para as experiências de aquecimento e geraçāo de corrente e fluxo no tokamak TCABR. Grande parte do trabalho dispensado no sentido da escolha dos melhores modos e frequêencias para operaçã̃o das antenas do TCABR está presente nesta tese. Este trabalho começou no início de 1997, quando o autor iniciou seus estudos sobre ondas de Alfvén, sob a orientação direta dos professores Artour Elfimov e Ricardo Galvão. Após um estágio de doís meses no Fusion Research Center, da University of Texas at Austin (EUA), no fim daquele ano, onde se familiarizou com o código toroidal disponivel naquele instituto, o autor iniciou o processo de adaptação do mesmo às necessidades do grupo. Algumas modificaçoes foram necessárias para a obtenção de resultados e a analise do código quanto a sua estrutura e limitaçōes. Hawia uma documentação extremamente pobre, o que demandou o estudo quase que por completo do funcionamento do código para se chegar às conclusöes acerca das mudanças que poderiam e deveriam ser introduzidas. Iniciado entẫo o uso mais intensivo deste código, a partir do incicio de 1998 , alteragoes foram sendo introduzidas a longo do trabalho, paralelamente à obtençäo e analise de resultados culminando corn os módulos de cálculo das forças ponderomotoras, no inicio deste ano.

Em suscinto resumo, o segundo capitulo apresenta a teoria de ondas de Alfvén. De inicio, é apresentada uma introdução básica até a derivaçăo do tensor dielétrico com termos cinéticos em primeira ordem. A relaçâo de dispersāo para plasmas homogêneos é 
obtida, a fim de servir de introdução à sefăto seguinte que mostra o efeito de conversäo de modos. Cálculos cilíndricos do acuecimento e o efeito de impurezas minoritárias seguem com a demonstraçäo dos modos globais.

No terceiro capítulo ê introduzido o cödigo bidimensional. As principais modificasōes provocadas pelos efettos toroidais são mostradas e analisadas.

No quarto capítulo, a teoria e os resultados principais dos cälculos das forças ponderomotoras säo apresentadas.

O quinto e ulltimo capitulo apresenta uma síntese dos resultados obtidos ao longo desta tese.

As unidades utilizadas estầo no sisterna Gaussiano (ou cgs). 


\section{TEORIA}

\subsection{Ondas de Alfvến - Introdução}

O método mais usado para o aquecinento auxiliar de plamas magneticamente con-

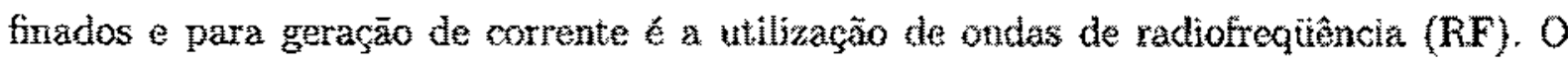
objetivo é converter a energia de ondas eletromatanéticas em energia térmica e luxo dass partículas no plasma. Entre as várias faixas de frequiência tillizadas com este propósito, este traballo explora as frequências abaixo da ressonảncia ciclotrônica da espécie princí pal de íons. Nas experiências de aquecimento e geraçăo de corrente via ondas de baixa freqüência, dois tipos de processos ocorrem. Em primeiro lugar, podem tomar lugar efeitos globais, com a excitação da onda feita por modos de cavidade, que dissipam via amorta cimento de Lanłau eletrônico, colisões e bombeamento magnético por tempo de trânsito (Trunstit Time magnetic Pumping), havendo uma transferencia de energia para o plasma como um todo. Uma outra forma de acoplamento da energia do modo eletromagnético é a conversão para uma onda Quase-Eletrostática de Alfvén (QEAW) ou onda Cinética de Alfvển (KAW), numa regiăo de ressonâneia especifica, que é amortecida, transferindo a energia para as particulas localmente (num primeiro momento). Indiretamente, outros mecanismos (colisöes e difusão) transferem esta energia para partículas fora da região das imediaçöes da ressonância. Entre os modos para os quais este tipo de acoplamento é relevante, pode-se destacar as ressonâncias ciclotrônica jônica, e ciclotrönica eletrônica e de Alfvén.

As ondas de Alfvén são particularmente interessantes pela chisponibilidade, a relativarnente baixo custo, de geradores de RF de alta potência, pela simplicidade do aparato para o lançamento das ondas (antenas), pela possibilitade de aquecer o centro da coluna de plasma e pela ausência de limites de densidade para operaçẩo de geraçĩo de corrente, 
tornando possivel alterar localmente o perfil da corrente de plasma com a conversäo de modos.

Descoberta por Hames Alfvén em 19427], quando tentava explicar a origem das manchas solares, as ondas de Alfuén servem de base para toda a teoria magnetohidrodinămica, e foram o primeiro exemplo de interaçāo das teorias eletromagnética e de fuidos. Alfuén percebeu que ura onda poderia se propagar num fluido incompressivel $(\vec{\nabla} \cdot \vec{v}=0)$, condutor perfeito $(\sigma=\infty)$ e imerso num forte campo magnético $\vec{B}_{0}$, este último fazendo o papel da forca restauradora tendo a densidade do plasma como inércia. Desprezando a resistividade, $\vec{J} \times \vec{B}$ e aJ/Jt na lei de Ohm $\overrightarrow{\vec{E}}=-\vec{y} \times \vec{B})$, as equacos de movimento sอ̃̊

$$
\begin{aligned}
\rho \frac{\partial \vec{v}}{\partial t} & =\frac{(\vec{\nabla} \times \vec{B}) \times \vec{B}}{4 \pi} \\
\frac{\partial \vec{B}}{\partial t} & =(\vec{B} \cdot \ddot{\nabla}) \ddot{v}-(\ddot{v} \cdot \vec{\nabla}) \vec{B} .
\end{aligned}
$$

Lineartzando as equaçoes acima e definindo as partes perturbadas com o sub-indice 1, supondo velocidade de equilibrio nula e desprezando os produtos de termos perturbados; chega-se a

$$
\begin{aligned}
\rho \frac{\partial \vec{v}_{1}}{\partial t} & =\frac{\left(\vec{\nabla} \times \vec{B}_{1}\right) \times \vec{B}}{4 \pi} \\
\frac{\partial \ddot{B}_{1}}{\partial t} & =(\vec{B} \cdot \vec{\nabla}) \vec{v}_{1} .
\end{aligned}
$$

Definindo o campo magnético de equilibrio na direcăo $z$ (Fig. 2.1), obtém-se como consequîncia um campo magnêtico e uma velocidade perturbados que săo independentes de $x$ e $y$ mas têm componentes apenas nestas dirę̧ö,

$$
\begin{aligned}
& p \frac{\partial v_{1 x, y}}{\partial t}=\frac{B_{0}}{4 \pi} \frac{\partial B_{1 x, y}}{\partial z} \\
& \frac{\partial B_{1 x, y}}{\partial t}=B_{0} \frac{\partial v_{1 x, y}}{\partial z}
\end{aligned}
$$




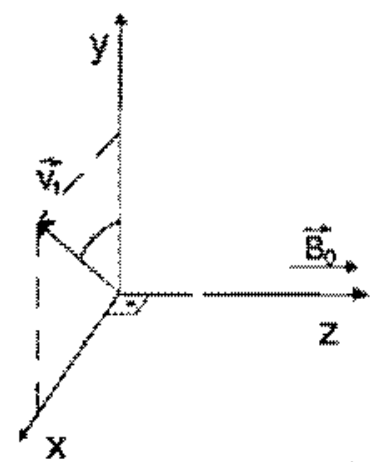

Figura 2.1. Tanto a velocidnde corno o campo magnẻtico perturbados estän na direçăo perpendicular no campo de equilitiro $\bar{B}_{0}$.

Combinando as duas equaçōes acima, obtém-se uma equaçäo de onda para a velocidade e campo perturbados na forma

$$
\left[\frac{\partial^{2}}{\partial t^{2}}-V_{\lambda}^{2} \frac{\partial^{2}}{\partial z^{2}}\right]\left[\begin{array}{c}
B_{1 x, y} \\
v_{1 x, y}
\end{array}\right]=0
$$

onde $V_{A}=B_{0} / \sqrt{4 \pi \rho}$ é a velocidade de propagação da onda transversal ao longo do campo magnético de equilibrio.

A soluçăo tem a forma oscilatória

$$
\left.B_{1 x}=|B| \exp i(k z-t) t\right)
$$

onde $t_{4}$ é a a trequência da onda, cuja velocidade de grupo $w / k=V_{A}$ é independente de w) (para qualquer valor de $h$ que o satisfaça). A Eq. (4) foca então natoma

$$
\left[w^{2}-b_{z}^{2} v_{A}^{2}\right]\left[\begin{array}{l}
B_{1 x, y} \\
v_{1 z, y}
\end{array}\right]=0
$$

sendo $z^{2}-k_{2}^{2} y_{A}=0$ a ralaça de dispersäo da onda de Alfvén.

Para sistemas de densidade inomogênea tratados pelas equaçōes $\mathrm{MHD}$, a ressonancia de Alvtu (ponto ou regita onde a Eq.(5) e satisfeita) aparece como una singularidade

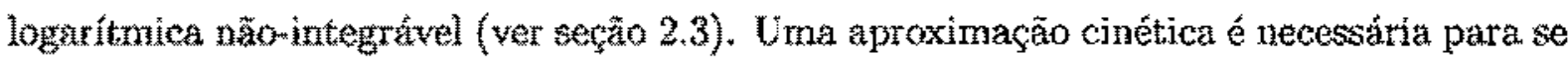
obter um quadro mais correto do que ocorre nas imediaçóes deste ponto. Isso é mostrado 


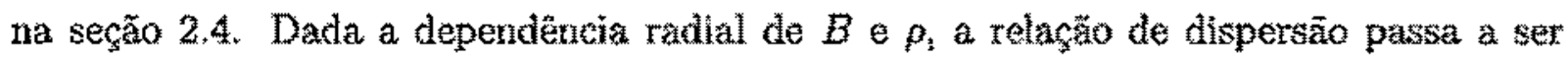
satisfeita numa faixa contínua de frequiênciass chamada de Contmuo de Alfvén.

Em tokamaks, as ondas de Alfvén säo geradas por antenas helicoidais (ou por estruturas que as simulem, como no caso do sistema de antenas do TCABR) e se propagam ao longo das linhas de campo magnético. A corrente supericial de uma antena de raio $b$, num modelo cilindrico simples pode ser descrita como

$$
J_{\zeta, \theta}=J_{\zeta, \theta}^{(M, N)} \delta(r-b) \exp [i(M \theta+N \zeta-i, \lambda)]
$$

onde $M$ e $N$ säo os números de onda da antena nas diregôes poloidal ( $\theta$ ) e toroidal ( $\zeta$ ), respectivamente, excitam um espectro de modos de Alfvén com seus números de onda

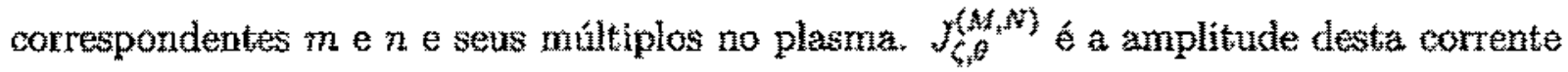
para cada um dos modos. Para o caso cilíndrico, os números $M$ e $m$ são os mesmos, mas no caso toroidal, devido a näo homogeneidade do campo magnético em $\theta$ e ao acoplamento dos modos poloidais vizinhos ao modo principal da antena, é necessário distingüir $M$ e 勧.

No passado, o aquecimento do plasma por ondas de Alfuén e a dispersä́o das ondas foram investigados intensivamente nos stellarators e no tokamak TCA (veja, por exemplo, Elfimov et al ${ }^{[8]}$ e Collins et al. $\left.{ }^{9}\right)$. Tipicamente, experimentos de aquecimento por ondas de Alvén em tokamaks pequenos sôno levados a cabo na faixa de baixas frequîenciáas, de 1 a 3 MHz. Antenas de grandes comprinentos de onda, que produzem números de onda toroidal na fajxa de $n=1$ a $n=4$ e números de onda poloidal $m= \pm 1$ e $m= \pm 2$, têm apresentado os melhores resultados (Stelarators $\mathrm{R}-0$ e $\mathrm{R}-3$, com modos locais, o no tokamak TCA, em Lauzanne, com modos globais). Os principais problemas destas experiềncias (no caso especifico do TCA) decorrem de um aumento incontrolável da densidade de plasma e impurezas pesadas parcialmente ionizadas, como oxigênio, sillio ou ferro, provenientes da parede da câmara durante o pulso de RF. 
Recentemente, o aquecimento eletrônico por ondas de Alfvén foi denonstrado no tokamak Phaedrus-T10. As experiencias de aquecimento no tokamak Phaedrus-T foram executadas na faixa de alta freqüência do espectro de Alfvĕ̉n, isto é, 7 a $9,2 \mathrm{MHz}$, com uma antena de curto comprimento de onda, que corresponde ao número de onda paralelo

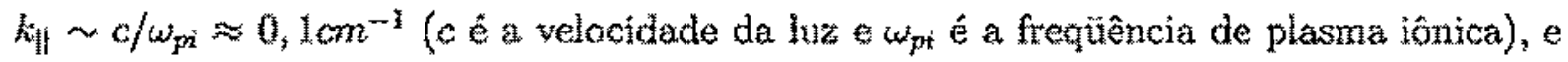
uma larga faixa de numeros de onda toroidal, aproximadamente de $n=6$ a $n=20$. Foi descoberto que procedimento de boronizaçäo, antes das descargas, proporciona melhores resultados para um controle da elevaçäo de densidade. Também é importante dizer que um esquema novo de blindagem da antena foi decisivo para a obtençäo dos resultados.

É provável que o aquecimento observado seja devido a ondas de Alfén Torsionais, em lugar de ondas Globais de Alfvén (GA), por causa da estrutura da antena e da pequena variaçäo na densidade durante a descarga de plasma. A antena do tokanak PhaedrusT" apresenta largo expectro, portanto é ineficiente quando usada para excitar os modos Globais de Alfún com nưmeses de modo polodal e toroidal (me en, respectivanente) bem definidos. Além disso, durante um disparo de $R F$, a densidade no Phaedrus- T era mantida num valor bastante baixo e aproximadamente constante no tempo. Sob estas condições, seria dif́cil produzir o valor da densidade específica requerido para a exctaçāo ressonante do modo Global de Alfvén. Por causa do limites impostos pela antena e propriedades do Phaedrus- $T_{1}$ a fraca interaçăo antena-plasma observada é um resultado esperado. En resumo, parece que as condigöes do tokarnak Phacdrus-T favorecem a excitação dos modos torsionals em lugar da onda Global de Alfvén.

Outro assunto relacionado com a excitaçäo com ondas de Alfén é o papel desempenhado pelas impurezas. Por exemplo, usualmente plasmas em tokamaks podem apresentar de $2 \%$. $4 \%$ de carbono tonizado. Populaçöes menores de outras impurezas também podern estar presentes. Então, é importante determinar os efeitos destas impurezas no 
comportamento das ondas de Alfyén no plasma.

Para resolver os problemas de aquecimento geraçāo de corrente via RF em plasmass colisionais, fe ustado o modelo magnetohidrodinâmico (MHD) $11,12,13]$ do plasma Em geral, plasmas de laboratónio ou espaciais săo fracamente colisionais, o que significa que

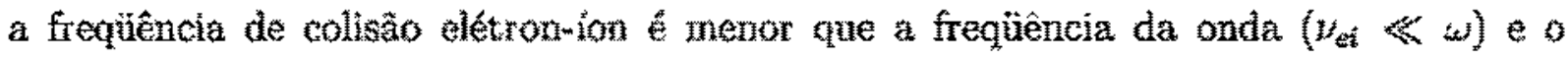
comprimento de onda paralelo a linhas de campo magnético $\left(2 \pi / k_{1}\right)$ é menor que o livre caminho medio dos eletrons $\left(2 \pi / k_{1}<w_{k} / \nu_{e}\right)$. Para estes plasmas, a maior parte dos efeitos da interaçăo ondutpartícula, como o aquecimento e geração de corrente por ondas, pode ser obtida atraves da soluçüo do sistema da equaçăo cinética,

$$
\frac{\partial F_{\alpha}}{\partial t}+\vec{v} \frac{\partial F_{\alpha \alpha}}{\partial \vec{r}}+\frac{e_{\alpha}}{m_{\alpha}}\left\{\ddot{E}+\frac{1}{c}[\ddot{v} \times \vec{B}]\right\} \frac{\partial F_{\alpha}}{\partial \vec{v}}=\hat{S} t\left\{F_{\alpha}\right\}
$$

e das equaçöes de Maxwell,

$$
\vec{\nabla} \times \vec{E}=-\frac{\partial \vec{B}}{\partial t} ; \quad \vec{\nabla} \times \vec{B}=\frac{1 \partial \vec{E}}{c t}+\frac{4 \pi}{c} \vec{J}
$$

Aqui, $F_{\alpha}, e_{\alpha}, m_{\alpha}$, sầ a função de distribuiçâ, a carga e a massa de ions ou elétrons, onde $\alpha$ especifica a espetcie; $\vec{r}, \vec{v}$, stäo os vetores de coordenadas do espaco de configuraçã $\vec{E}, \vec{B}, \vec{j}$, são os campos elétrico e magnético e a densidade de corrente; $c$ é a velocidade da luz no vácuo e $\hat{S} t\{F\}$ é o operador de colisto na torma de Landaullis!.

Em geometrias de campo magnetico complicadas (toroidal, por exemplo) a funçào de distribução $F=F(t, \vec{r}, \vec{v})$ depende de sete variaveis é necessăio incluir o efeito

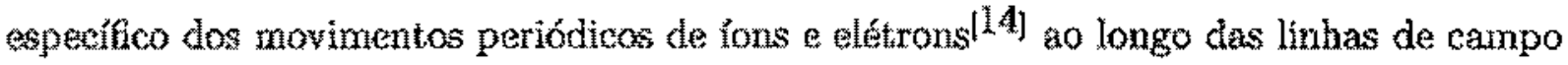
magnéticas. A solução deste problema näo-linear geral é uma tarefa formidável no campo das equaçōes de Vlasov-Maxwel. Para resolver este problema, alguns passos devem ser tomados:

- No primeiro passo, a equação de Vlasov (Eq.(7)) é resolvida em uma geometria adequada e o tensor dielétrico é calculado. Assim, a conexăo entre as lutuaģoes da 
densidade de corrente e do campo eletromagnético no plasma pode ser determinada. Isso é mostrado na seção 2.2.

- No próximo passo, levando em conta as expressöes analiticas obtidas para o tensor dielétrico, as equaçōes de Maxwell são resolvidas para se estudar a estrutura dos campos da onda e avaliar a potência dissipada pela onda. Tais cálculos sã̃o desenvolvidos de uma foma mais simples nas seçóes 2.3 e 2.4 . Os resultados numéricos sẵo mostrados e discutidos, primeiro numa configuraçăo cilíndrica (Seçäo 2.5) e depois em geometria toroidal (Capitulo 3).

- Finalmente, calculando a média da equação de Vlasov (com o operador de colisão cinético) sobre as oscilaşöes de onda e sobre ts superficies magnéticas, a geração de corrente e fluxos pode ser calculada. Tais cálculos sāo realizados no capítulo 4 .

Neste capítulo é mostrado o cálculo do tensor dielétrico em geonetria plana; é dẹrivada a relaçấo de dispersão para ondas de Alfvén e é demonstrado o câlculo do campo eletromagnético para um perfil inomogêneo de densidade (ressonância de Alfyén localizada). Tambẻm sâo mostrados neste capítulo resultados obtidos na análise numérica do efeito de impurezas no plasma. Os cálculos dos campos das ondas de Alfvén e potência dissipada foram realizados com um código unidimensional desenvolvido por Dmitrieva et a ${ }^{15,16]}$. Este cotdigo é apropriadio para o estudo das ondas de Alvến Globais e ondas de Alfvén Torsionais, dentro do escopo deste capitulo, porque inclui os efeitos de impurezas e um perfil de corrente axial. Os cálculos aqui apresentados são para os casos mais simples, não ineluindo contribuiçồ de maior ordem do efeito de raio de Larmor finito e da frequiêneia ciclotrồnica iônica; todavia, os passos para se realizar tais incrementos sâo mostrados. 


\subsection{Equaçōes Básicas, Tensor Dielétrico}

Para descrever a ação das ondas no plasma corn as equaçöes de Maxwell, é preciso compor o ente matemático atraves do qual ê modelada a resposta do meio à presença dos campos eletromagnéticos. Tal ente é chamado de tensor dielétrico".

Das Equaçöes de Maxwell linearizadas (Eq. 3), a onda eletromagnética num meio qualquer pode ser descrita na forma

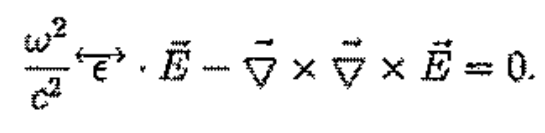

As informaçöes sobre a reeposta do meio material, contidas no tensor dielétrico $\vec{t}$. e as informaçôes sobre a geometria do problema permitem compor o perfil dos campos eletromagnéticos dentro do plasma e entäo calcular todas as quantidades pertinentes ao

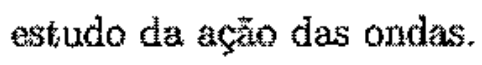

Abaixo é mostrada a derivação do tensor dielatrico a partir da equaçäo de Vlasov. E feita a hipótese de caue os lons näo se movem (na direção paralela ao campo magnético) sob a açäo dos campos de $R F$, aproximaçäo válide devido ao pequeno valor da razäo $m_{c} / m_{i}$, onde $m_{i} m_{i}$ sä́ a massas dos elétroms a dos íons, respectivamente.

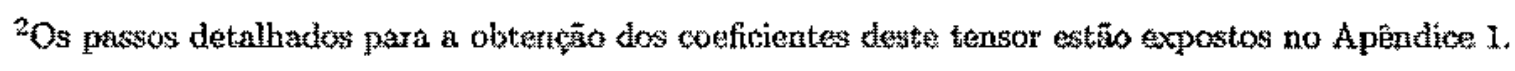




\subsubsection{Funģão Distribuiçäo no Espaço de Velocidades}

Linearizando a Equaçäo de Vlasov (Eq.(7)) (que é aquaçăo cinética quando o termo colisional $\widehat{S} t\left\{F_{\alpha}\right\}=0$ ), fazendo as transformaçoes para coordenadas espaciais cilíndricas

$$
X=r \cos \theta, \quad Y=r \sin \theta, \quad Z=z_{\text {, }}
$$

e definindo um sistema de coordenadas baseado no campo magnético de equilibrio e com o ângulo $\sigma$ (definido a partir da direção deste campo $\ddot{B}_{0}=B_{4} \hat{e}_{2}$ ), mais as direcoues paralela e perpenducular a $\vec{B}_{0}$.

$$
v_{r}=u_{1} \cos \sigma, \quad v_{0}=v_{1} \sin \sigma_{1}, \quad v_{z}=v_{1}
$$

obtém-se a expressäo linearizada

$$
\begin{aligned}
& \frac{\partial F_{\alpha}}{\partial t}+v_{\perp} \cos \sigma \frac{\partial F_{\alpha}}{\partial r}+\frac{v_{1}}{r} \sin \sigma \frac{\partial F_{\alpha}}{\partial \theta}+v_{\|} \frac{\partial F_{\alpha}}{\partial z}-\left(\omega_{c}+\frac{v_{1}}{r} \sin \sigma\right) \frac{\partial F_{\alpha \alpha}}{\partial t}+ \\
& \frac{e_{\alpha}}{m_{\alpha}}\left\{\left[\left(E_{r}-\frac{v_{1}}{c} B_{0}\right) \cos \sigma+\left(E_{j}+\frac{\nu_{1}}{c} B_{r}\right) \sin \sigma\right] \frac{\partial F_{\alpha}}{\partial v_{L}}+\right. \\
& {\left[\frac{\nu_{1}}{c}\left(B_{0} \cos \sigma-B_{s} \sin \sigma\right)+E_{*}\right] \frac{\partial F_{\alpha}}{\partial \nu_{1}}+} \\
& \left.\left.\left[\left(E_{\theta}+\frac{\eta_{11}}{c} B_{r}\right) \cos \theta-\left(E_{r}-\frac{q_{1}}{c} B_{0}\right) \sin \sigma\right] \frac{\partial F_{\alpha}}{v_{L} \partial \alpha}\right\}=S t F_{a}\right\} \text {. }
\end{aligned}
$$

Compondo um desenvolvimento en série para a perturbação em primeira ordem da funçäo de distributição das particulas,

$$
\tilde{f}=\left\{f_{0}+\sum_{l, f_{0}}\left[f_{F_{i}} \cos (l \sigma)+f_{0, t} \sin (l \sigma)\right]\right\} \exp (m \theta+k z-\Omega t)
$$

e gubstituindo a expressä̃o acima no desenvolvimento em ondas planas, obtém-se axpressão tokal de $F$, em primeira ordem, no espaço de configuraçăo e de velocílades:

$$
F=F_{M}+F_{b} \sin \theta+F_{r} \cos \alpha+\left(f_{b}+f_{r} \cos \theta+f_{b} \sin \sigma\right) \exp z\left(m \theta+k_{z} z-\Omega t\right)
$$

onde $F_{M}$ é a hunçăo de distribuç̧āo maxwelliana de equilibrio, $F_{b}$ é a perturbaçāo em primeira ordem na direçäo binormal (tarmo mais apropriado que "perpendicular" para configuraçöes com curvatura\} e $F_{\gamma}$ e a perturbaçäo em primeira ordem na direção radial. 
Substituindo esta última na E. (10) e fazendo a hipotase do operador colstonal de Krook,

$$
\widehat{S} t\left\{F_{\alpha}\right\}=\nu_{e} F_{e}
$$

que corresponde substituição $\omega \rightarrow \Omega-i \nu_{\mathrm{e} f}$. Supondo que o raio de Larmor é muito menor que as inomogeneidades na direçäo radial, e mais alguma álgebra, chega-se ao conjunto de equaçöes para a funçäo distribuiçāo perturbada en primeira ordem:

$$
\begin{aligned}
& \left(k_{0} v_{1}-\omega\right) f_{0}+\frac{v_{1}}{2}\left(\frac{d f_{r}}{d r}+\frac{f_{r}}{r}+i \frac{m}{r} f_{b}\right)= \\
& -\frac{e_{\alpha}}{m_{\alpha}}\left[E_{z} \frac{\partial F_{M \alpha}}{\partial \nu_{1}}+\frac{E_{0}}{\omega_{r}} \frac{\partial}{\partial r}\left(\frac{F_{M \alpha}}{2}+\frac{\nu_{1}}{2} \frac{\partial F_{M \alpha}}{\partial \nu_{\perp}}\right)+\frac{\nu_{\|}}{2 c \nu_{\perp}} B_{r 1} F_{b}\right] \\
& \omega_{t} f_{b}=-i\left(\omega-k_{\|} v_{\|}\right) f_{t}+v_{z} \frac{\partial f_{0}}{\partial r}+\frac{p_{\alpha}}{m_{\alpha Q}}\left[E_{t} \frac{\partial F_{M a}}{\partial v_{1}}-\frac{B_{z}}{c} F_{t}\right] \\
& \omega_{c} f_{r}=i\left(\omega-k_{n} v_{\|}\right) f_{b}-i k_{b} v_{\perp} f_{0}-\frac{e_{\alpha}}{m_{\alpha}}\left[E_{\theta} \frac{\partial F_{M \alpha}}{\partial v_{\perp}}+\frac{E_{z}}{c} \frac{\partial F_{b}}{\partial v_{\perp}}\right]
\end{aligned}
$$

A densidade de corrente oscilatória (de RF) em um plasma, que é calculada pela integral $\int f_{\alpha} \tilde{w}^{3} v$, é definida nas direçoes radial, binormal e paralela pelas expressoues

$$
\begin{aligned}
& \hat{f}_{r}=\sum_{\alpha} e_{\alpha} \int_{0}^{\infty} d \sigma \cos \sigma \int_{-\infty}^{\infty} d v_{i} \int_{0}^{\infty} v_{\perp}^{2} \tilde{f}_{\alpha} d v_{\perp}=\pi \sum_{\alpha} e_{\alpha} \int_{-\infty}^{\infty} d v_{1} \int_{0}^{\infty} v_{\perp}^{2} f_{r}^{\alpha} d v_{L} \\
& u_{k}=\sum_{\alpha} e_{\alpha} \int_{0}^{\infty} d \sigma \sin \sigma \int_{-\infty}^{\infty} d v_{\|} \int_{0}^{\infty} v_{1}^{2} f_{\alpha} d v_{\perp}=\pi \sum_{\alpha} e_{\alpha} \int_{-\infty}^{\infty} d v_{\|} \int_{0}^{\infty} v_{\perp}^{2} f_{b}^{\alpha} d v_{\perp} \\
& f_{\|}=\sum_{\alpha} e_{\alpha} \int_{0}^{\infty} v_{\|} d \sigma \int_{-\infty}^{\infty} d v_{\|} \int_{0}^{\infty} v_{\perp} \tilde{f}_{\alpha} d v_{\perp}=2 \% \sum_{\alpha} e_{\alpha} \int_{-\infty}^{\infty} v_{\|} d v_{\|} \int_{0}^{\infty} v_{\perp} f_{0}^{\alpha} d v_{1}
\end{aligned}
$$

onde o somatớrio ags sobre todas as espécies carregadas no plasma.

Finalmente, empregando a relaçấo entre a corrente oscilatória e campo elétrico,

$$
4 \pi j_{i}=-i n \epsilon_{i j} E_{3}
$$


chega-se às componentes do operador tensor dielétrico:

$$
\begin{aligned}
& \hat{k}_{11}=\sum_{\alpha} \frac{w_{F_{0}}^{2}}{w_{c \alpha}^{2}}\left[\left(1-\frac{w^{2}}{\omega_{c \alpha}^{2}}\right)^{-1}+2 \frac{k_{b}^{2} v_{r \alpha}^{2}}{\omega^{2}}\left(\Lambda_{\alpha}-1\right)\right]
\end{aligned}
$$

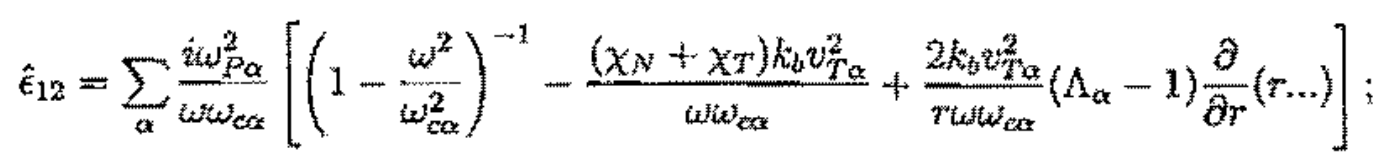

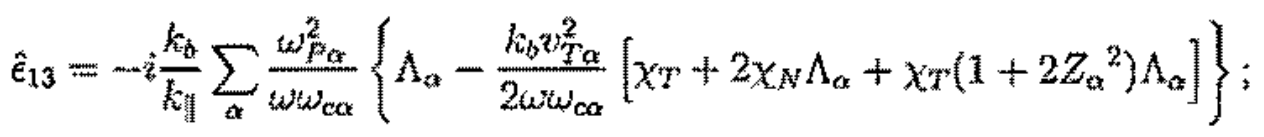

$$
\begin{aligned}
& \hat{\epsilon}_{21}=-\hat{\epsilon}_{12}-i \sum_{\alpha} \frac{\omega_{P \alpha}^{2} h_{b} \omega_{\alpha \alpha}^{2}}{\omega^{2}}\left\{\frac{4}{r}\left(\Lambda_{\alpha}-1\right)-2 \chi_{N} \Lambda_{\alpha}-\chi_{T}\left[\left(1+2 Z_{\alpha}^{2}\right) \Lambda_{\alpha}-1\right]\right\}
\end{aligned}
$$

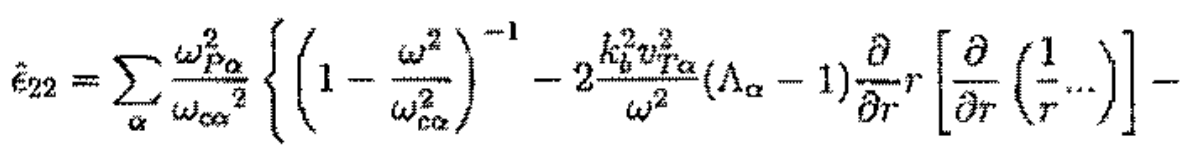

$$
\begin{aligned}
& \left.\frac{\chi_{N} v_{T \alpha}^{2}}{r \omega^{2}}\left[1+2\left(\Lambda_{\alpha x}-1\right) \frac{\partial}{\partial r}\left(r_{\alpha}\right)\right]-\frac{\chi_{T} v_{T \alpha}^{2}}{\eta v^{2}}\left[1-\left(1-\left(1+2 Z_{\alpha}^{2}\right) \Lambda_{\alpha \alpha}\right) \frac{\partial}{\partial r}(r . .)\right]\right\}
\end{aligned}
$$

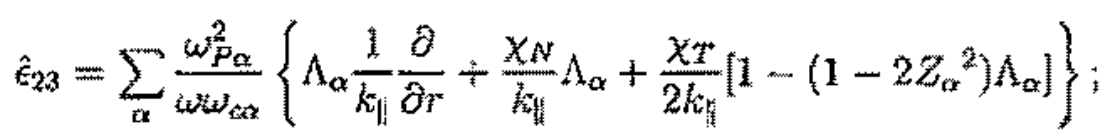

$$
\begin{aligned}
& \hat{\epsilon}_{31}=-\mathrm{E}_{3}
\end{aligned}
$$

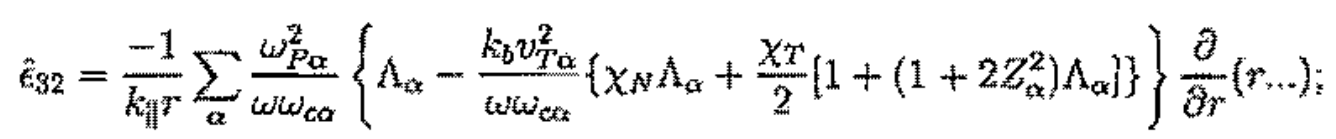

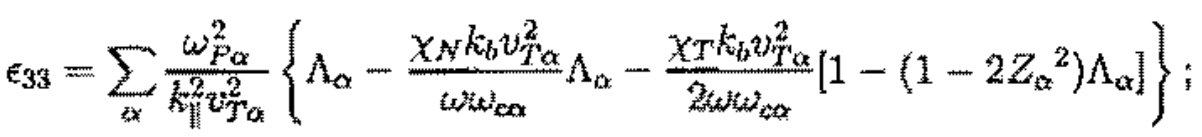

onde $\omega_{P \alpha}^{2}=4 \pi N_{0 \alpha} e_{\alpha}^{2} / m_{\alpha \alpha}$ é a freqüência de plasma, onde $N_{0 \alpha}$ é a densidade central de particulas, $e_{\alpha}$ e $M_{\alpha}$ săo massa e carga das particulas. Os números 1,2 e 3 significam, respectivamente as projeços radial, binomal e paralea.

$$
\Lambda_{\alpha x}=1+\sqrt{\pi} Z_{\alpha \alpha} W\left(Z_{\alpha \alpha}\right), \quad Z_{\alpha \alpha}=\frac{\xi}{\sqrt{2} k_{\|} v_{T \alpha}}
$$


onde

$$
W\left(Z_{\alpha}\right)=\frac{-i}{\pi} \int_{-\infty}^{\infty} \frac{\exp \left(-Z_{\alpha}^{2}\right)}{t-Z_{\alpha}} d t=\exp \left(-Z_{\alpha}^{z}\right)\left[1+\frac{2 i}{\sqrt{\pi}} \int_{0}^{Z_{\alpha}} \exp \left(t^{2}\right) d t\right],
$$

é a funçâo de dispersäo do plasma, e os parâmetros de inomogeneidade radial $\chi_{1}, \chi_{N}, \chi_{T}$ são definidos como

$$
\chi_{N}=\frac{\partial}{\partial r} \ln N_{0}, \quad \chi_{T}=\frac{\partial}{\partial r} \ln T_{\alpha} .
$$

Estes săo termos da derivada logaritmica da densidade de equilíbrio, $N_{0}$, e da temperatura $T_{\alpha}$ das particulas do plasma.

\subsection{Relação de Dispersão para Ondas de Alfvén (Plasma Ho- mogêneo)}

Nesta seção é mostrada a obtenção das expressōes dos campos perturbados pela açäo da onda no plasma a partir da equação de dispersão. Os dois ramos da soluçăo da equação de dispersâo säo mostrados com o intuito de ilustrar a proximidade das soluçōes en torno da ressonáncia de Alfvén. Na seçăo seguinte o fenòmeno da conversāo de modos é explicado e calculado com mais detalhes.

Das Eq̣s.(9) e (17), obtém-se, através da aproximação da óptica geométrica,

$$
k^{2} \vec{E}-\vec{k}(\vec{k} \cdot \vec{E})=\frac{4 \pi i \omega}{c^{2}} \vec{J}
$$

onde

$$
k^{2}=k_{i}^{2}+k_{1}^{2} .
$$




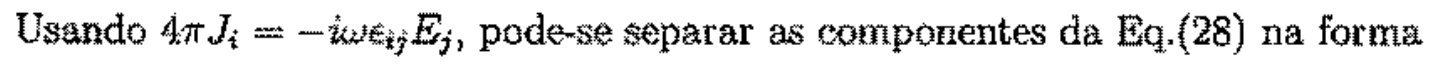

$$
\begin{aligned}
& k_{7 /}^{2} E_{x}-k_{1} k_{i z} E_{z}=\frac{\omega^{2}}{c^{2}}\left(c_{11} E_{z}+\epsilon_{12} E_{y}\right) \\
& k^{2} E_{y}=\frac{\xi^{2}}{c^{2}}\left(-\epsilon_{12} E_{x}+\epsilon_{22} E_{x}+\epsilon_{23} E_{y}\right) \\
& k^{2} E_{z}-\left(k_{y /} E_{z}+k_{1} E_{x}\right)=\frac{\omega^{2}}{c^{2}}\left(-\epsilon_{23} E_{y}+\epsilon_{33} E_{z}\right)
\end{aligned}
$$

Da Eq.(30a), obtém-se a expressão

$$
E_{x}=\frac{\frac{u^{2}}{Z^{2}} \epsilon_{12} E_{y}+k_{1} h_{y} E_{z}}{\left(k_{/ 1}^{2}-\frac{w^{2}}{c^{2}} \epsilon_{11}\right)}
$$

Inserindo (31) $\mathrm{em}(30 \mathrm{~b})$, obtém-se

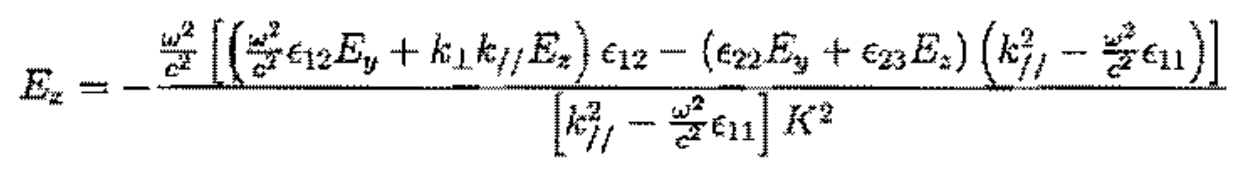

e

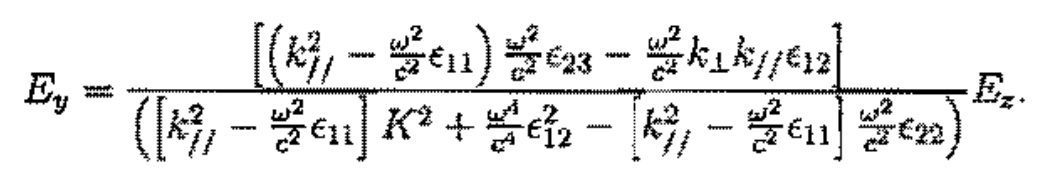

Inserindo (31) em (300), obtém-se

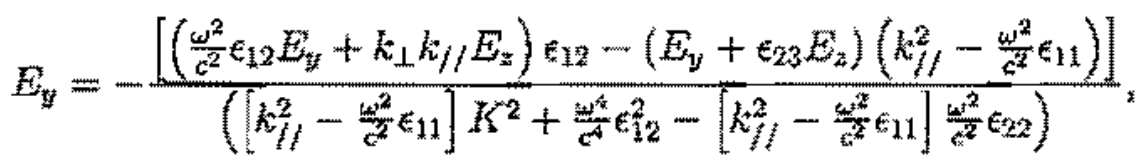

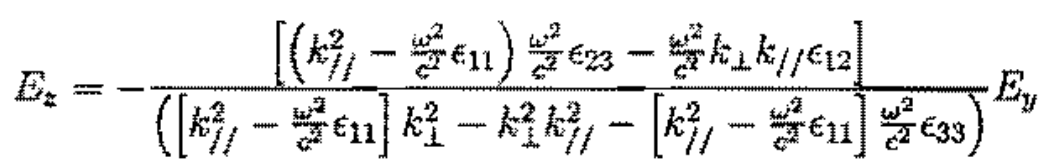

e

$$
\begin{aligned}
& {\left[k_{/ /}^{2}-\frac{\omega^{2}}{c^{2}} \epsilon_{11}\right] k^{2}+\frac{w^{4}}{c^{4}} \epsilon_{12}^{2}-\left[k_{/ 1}^{2}-\frac{\omega^{2}}{c^{2}} \epsilon_{11}\right] \frac{\omega^{2}}{c^{2}} \epsilon_{22}=}
\end{aligned}
$$

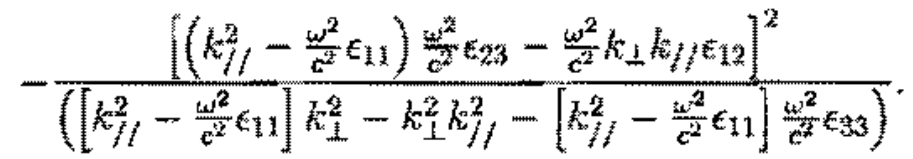

Utilizando a condiyăo de velocidade de fase garalela muito maior que a velocidade térmica $\left(\hat{b}_{i} / t_{t}>>v_{T i}\right)$, obtém se a expressäo

$$
\begin{aligned}
& k_{\perp}^{2}=\frac{\left(\frac{\phi^{2}}{c^{2}} \varepsilon_{12} \epsilon_{12}-k_{11}^{2}\left(1-\frac{\epsilon_{22}}{\epsilon_{11}}\right)-D\left(\frac{\epsilon_{3 \pi}}{\epsilon_{11}}-\frac{\epsilon_{21}}{\epsilon_{11}}\right)\right)}{2} \pm
\end{aligned}
$$

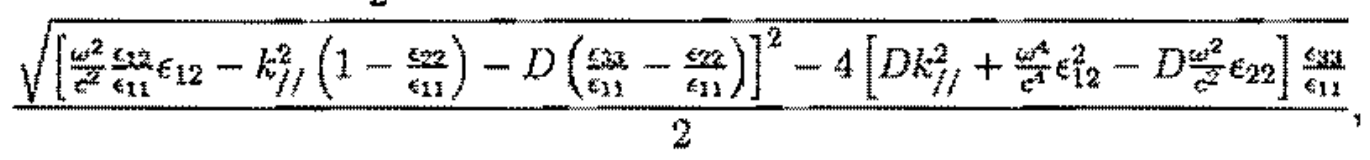


onde $D=\left[k_{1 /}^{2}-\frac{y_{2}^{2}}{c^{2}} \epsilon_{11}\right]$

Fazendo $\epsilon_{22} \cong \epsilon_{11}$, válido quando supöe-se $\frac{k_{w_{2 \alpha}^{2}}^{2}}{w_{c \alpha}^{2}}=k_{1}^{2} p_{\alpha}<<1$ (ver expressẫo do tensor dielétrico), sendo $\rho_{\alpha}$ o raio de Larmor para a espécie $\alpha$, e incluindo apenas os termos proporalonais a $\frac{\epsilon_{33}}{\epsilon_{13}}$

$$
h_{-}^{2}=-\frac{D}{2} \frac{\epsilon_{33}}{\epsilon_{11}} \pm \frac{D}{2} \frac{\epsilon_{33}}{\varepsilon_{11}} \sqrt{1-\frac{4}{D^{2}}\left[D k_{11}^{2}+\frac{w^{4}}{c^{4}} \epsilon_{2}^{2}-D \frac{w^{2}}{c^{2}} \varepsilon_{11}\right] \frac{\epsilon_{11}}{\epsilon_{33}}}
$$

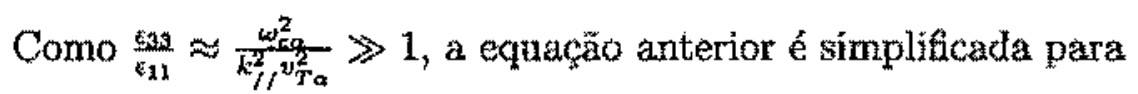

$$
k_{1}^{2}=\frac{D}{2} \frac{\epsilon_{33}}{\epsilon_{11}}\left\{-1 \pm\left(1-\frac{2}{D}\left[k_{/ 1}^{2}+\frac{\omega^{x}}{c^{4}} \frac{\epsilon_{12}^{2}}{D}-\frac{\omega^{2}}{c^{2}} \epsilon_{11}\right] \frac{\epsilon_{11}}{\epsilon_{33}}\right)\right\} .
$$

Para o ramo com sinal negativo, a solução é dada na forma das ondas de Alfón Ginéticas (KAW on SQEAW)

$$
k_{1}^{2}=-D \frac{\epsilon_{33}}{\epsilon_{11}}+D+\frac{\omega^{4}}{c^{4}} \frac{\epsilon_{12}^{2}}{D} \cong \frac{\epsilon_{33}}{\epsilon_{11}}\left[\frac{\omega^{2}}{c^{2}} \epsilon_{11}-k_{/ /}^{2}\right]
$$

e no ramo com sinal positivo, apresentam-se as soluçoes para as ondas de Alfuẻn Rápidas $(\mathrm{FAW} \text { or } \mathrm{EMSW})^{\mathrm{A}}$ :

$$
k_{\perp}^{2}=-k_{/ /}^{2}+\frac{\omega^{2}}{c^{2}} \epsilon_{11}-\frac{\omega^{4}}{c^{4}} \frac{\epsilon_{12}^{2}}{D}=-k_{/ /}^{2}+\frac{\omega^{2}}{c^{2}} \epsilon_{11}-\frac{\omega^{4}}{c^{4}} \frac{\epsilon_{12}^{2}}{\left[\frac{\omega^{2}}{c^{2}} \epsilon_{11}-k_{/ /}^{2}\right]}
$$

Nurna primeira aproximaçäo, para $\frac{\omega}{w_{\epsilon \alpha}}<<1, \epsilon_{\mathrm{n}}$ se resume a $c^{2} / V_{A}^{2}$ e $D=0$ é a relaçăo de dispersão da onda de Alfvén, ja vista na introdução deste capitulo. Para estas soluçōes, a expressẫo para onda rápida (soluģăo MHD) diverge e năo ê apropriada para a descrição do comportamento dos campos próximo a $D=0$ (resonência de Alfvén). É necessário se levar em conta efeitos cinéticos, como um valor finito para pa. para entender o que ocorre próximo à ressonância.

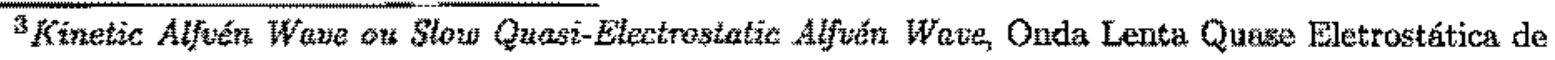
Alfyén, para o caso w/la

${ }^{4}$ Fust Alfuén Wave or Fast Magnetosonic Waute, Onda Râpida Magnetcacútica de Alfyén.
} 
Variando a densidade de partículas do plasma, simulando então um plasma inomogếneo, ́a possível superpor as duas soluçōes (Onda Rápida e Onda Cinética) para verificar que na ressonância ambas tem a mesma ordem de grandeza, permitindo o efeito de Conversãa de Modos (ver Fig. 2.2). O efeito de conversão de modos, que ocorre em plasmas ínomogểneos, é estudado em detalkes na próxima seção.

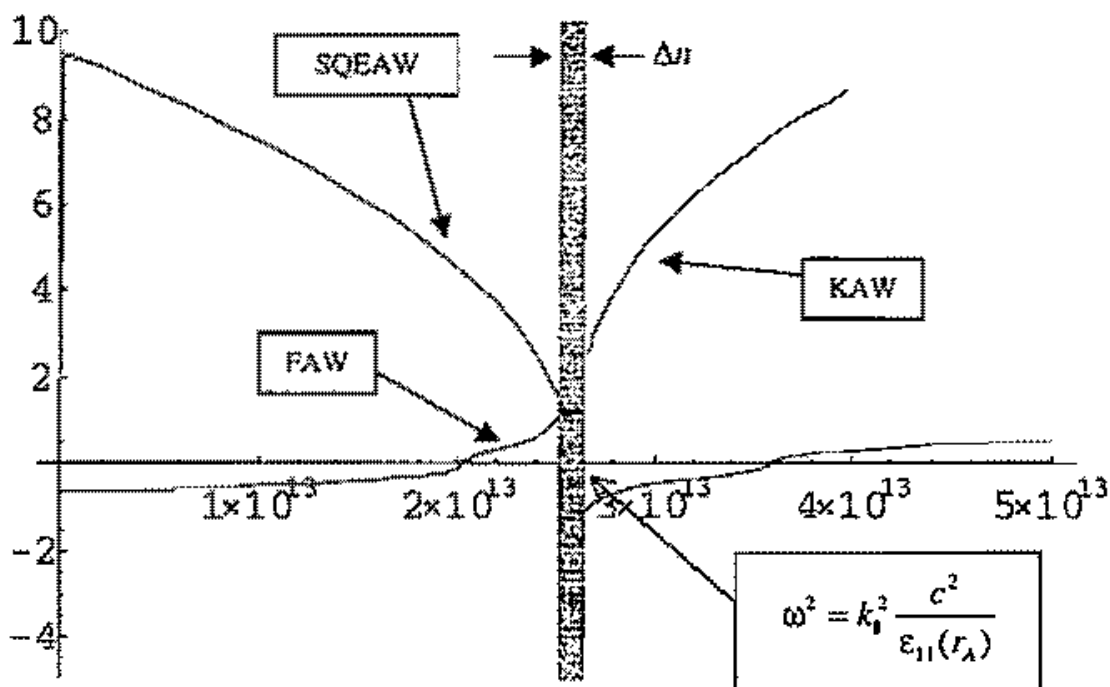

Fig. 2.2 Esquema dos modos do plasma, fora da ressonância local de Alfvén lesta última na regiăo em cinza, com espessura $\Delta n$,en torno da ressonância de Alfyén $(D=0)$ onde as soluçoss com aproximação de óptica geomêtrica vão så̃ välidas), Soluçöes ta Eq. (38) de dispersão para SQEAW (caso de plasma frio, $w / k_{\|} \gg v_{T}$ ) e FAW (MHD), para as condiçôes do TCABR e com frequência $f=4.3 \mathrm{MHz}, n=4$, e densidade eletrônica variando no intervalo $n_{e}=0-5,10^{13} \mathrm{~cm}^{-3}$. Podere

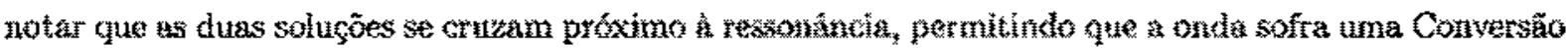
de Modos. 


\subsection{Ondas de Alfvén em Plasmas Cilíndricos Inomogêneos e Efeito de Conversão de Modos}

$\mathrm{Na}$ seçãa anterior é mostrado que o modelo MHD com plasma homogêneo däo dascreve adequadamente as soluçöes en tomo da ressonância de Alfvén. Aqui, o modelo completo das ondas de Alfvén, desenvolvido nas Refs. $[17,18,19]$, é usado para analisar a estrutura dos campos de uma onda en uma coluna inomogênea de plasma cilindrico de raio

Este modelo de plasma cilíndrico é razoável para tokamaks (ou arcos da Coroa Solar) com baixa pressão de plasma $(\beta<1)$ e um parâmetro de toroidicidade pequeno (a/R 1 , onde a o raio menor e Ré o raio maior do tokamak). O plasma é considerado magnetizado, de forma que o raio de Larmor dos elétron é menor que a inomogeneidade

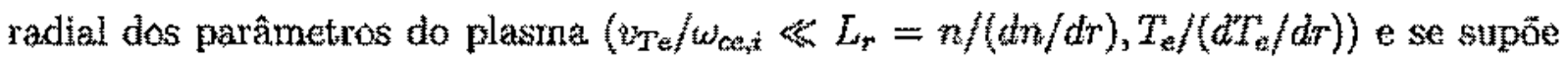
íns frios $\left(T_{\mathfrak{i}} \ll T_{e}\right)$. Uma antena helicoidal com raio $b$ é situada na regiâo de vácuo entre o plasma e una parede condutora com raio c. Este modelo de plasma é bem similar ao modelo discutido nas Reis. $[20,11,21]$. A estrutura do campo de RF produzido no plasma por uma antena exterma é descrita pelas equaçöes de Maxwell-Vlasov. Nestas equaçōes, a corrente oscilatória induzida por uma onda plana pode ser expressa pelo tensor dielétrico e pelo campo elétrico de $\mathrm{RF}$, na aproximaçäo línear:

$$
\tilde{j}_{s}=-(1 \mathrm{~N} / 4 \pi) \epsilon_{s q} \vec{E}_{q}, \quad \tilde{E}_{r \phi z}=E_{r, \theta, z}(r) \operatorname{expi}\left(m \theta+k_{z z} z-\Omega t\right)
$$

Os números de onda poloidal $(m \neq 0)$ e axial $\left(k_{z} \neq 0\right)$ são escolhidos como năonulos. Na aproximaçäo eikonal $\{17,20,22\}$, a dependencia radial dos campos de $\mathrm{FF}$ é representada na forma das componentes (radial, binomal e paralela)

$$
E_{r_{r}, b_{n} \|}=\mathcal{E}_{r, b_{1}, \|} \exp \left(\hat{i} \int_{0}^{r} k_{r} d r\right)
$$


nas quais a amplitude $\varepsilon_{r, b, 1}$ é radialmente uniforme $\left(\left|d \varepsilon_{r, b, j} / d r\right|<<\left|k_{r} \varepsilon_{r, b, \|}\right|\right)$.

Podem ser oblidos dois ramos para as oscilaçōes de plasma (como visto na seção anterior, para a Eq.(37)): Onda Rápida (FAW) e onda Cinética (KAW/SOEAW). As relaçöes de dispersäo (modificadas) săo

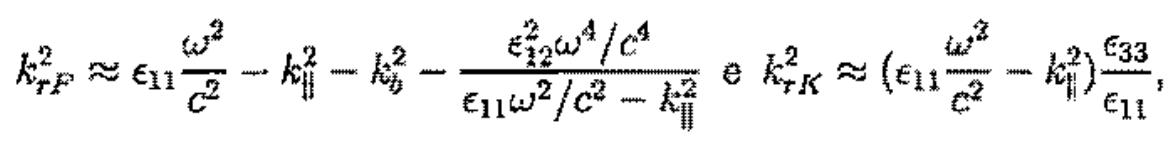

respectivamente. Estas relaçōes sẫo vălidas longe da ressonància local de Alfvên (AWR), $\mathrm{r}-$ $r_{A} \gg c_{s} \omega_{c i}$ (onde $c_{s}=\sqrt{T_{e} / \eta_{i}}, T_{e}$ em eV), é a velocidade ion-acústica e $r_{A}$ é a posiçấo radial da ressonância de Alfvén (onde $D=k_{i}^{2}-\epsilon_{1} \omega^{2} / c^{2}=0$ ). Estas quantidades são obtilas das Eas 40 e 41, fazendo-se $k_{1}^{2}=k_{b}^{2}+k_{r}^{2}$ e $k_{1}^{2}>>k_{b}^{2}$. Para apresentar solução do problema de uma forma completa, deve-se encontrar a soluço das equaçöes de Maxwell válida no entorno da ressonância de Alfvén (AWR) local, e casá-la con o resultado da aproximação de ôptica geométrica nas Eqa. $(42,43)$ longe da AWR local $\left(\left|r-r_{A}\right|\right.$ s $\left.c_{s} / w_{c i}\right)$.

Nas equaçōes acima, as componentes cinêticas do tensor dielétrico são apresentadas na aproximacăo local:

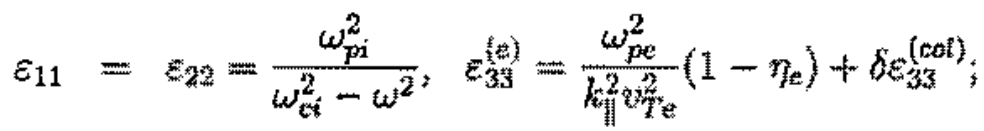

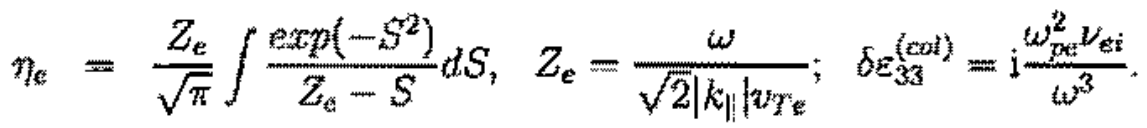

Aqui, além do amortecimento de Landat, a dissipagäo colisional fraca das ondas

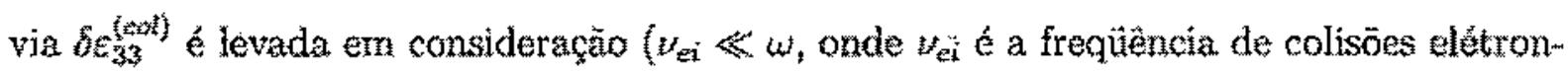
Son, e $\omega$ é a frequêtncia das ondas). A dissipação é muito importante próximo à borda do plasma, onde a temperatura eletronica é baixa e amortecimento de Landau das ondas de Alfvén é muito fraco. O termo colisional aparece quando é feito o limite $w_{1} / k_{1} \gg v_{T e}$, quando a integraçăo da funçāo de dispersão do plasma $\left(n_{e}\right)$ resulta em

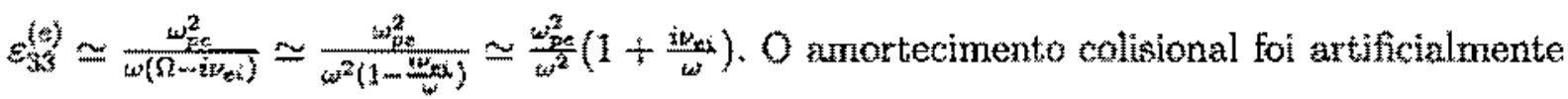


introduzido no modelo substituindo $\omega$ no tensor dielétrico por $\Omega-i \nu$, onde $\Omega$ a entăo frequiência real da fonte de RF. Esta transformaçäo da freqü̉encia vem da representaçă de Krook do operador colisional. As colisöes são usadas para remover ondas de Alfvén de pequeno comprimento de onda na periferia da coluna de plasma, ou ondas de Bernstein na resson ciclotrônica iônica.

\subsubsection{Efeito de Conversä́o de Modos}

Com o intuito de evitar fórmulas matemáticas complexas para a análise da conversão de modos, um modelo de plasma mais simplas ê usado. $O$ efeito Hall e o campo magnético poloidal são considerados pequenos $\left(B_{0} \approx 0\right)$, de tal forma que pode ser escolhida a forma diagonal do tensor dieletrito na Eq.(4) $\left(\varepsilon_{12}=\varepsilon_{21}=\varepsilon_{13}=\varepsilon_{31}=0\right)$. A densidade $\mathrm{e}$ a temperatura do plasma são consideradas homogeneas ao longo das linhas de campo magnếtico e trapezoidais ao longo do raio com uma pequena queda, $\delta n=n_{0}\left(a_{1}-a\right) / L_{r}$, na borda do plasma:

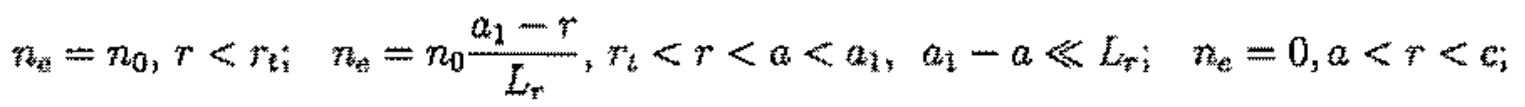

onde $L_{r}=a_{1}-r_{\imath}$ é o parămetro caracteríticico da inomogeneidade do plasma. Depois, supöe-se que a frequência de RF esteja no contínuo de Alf́vén, $k_{z} c_{A}(0)<\omega<k_{*} c_{A}\left(a_{1}\right)$ o que significa que o ponto de conversäo deveria estar na região da inomogeneidade do plasma $\left(r_{t}<r_{A}<a\right)$ 


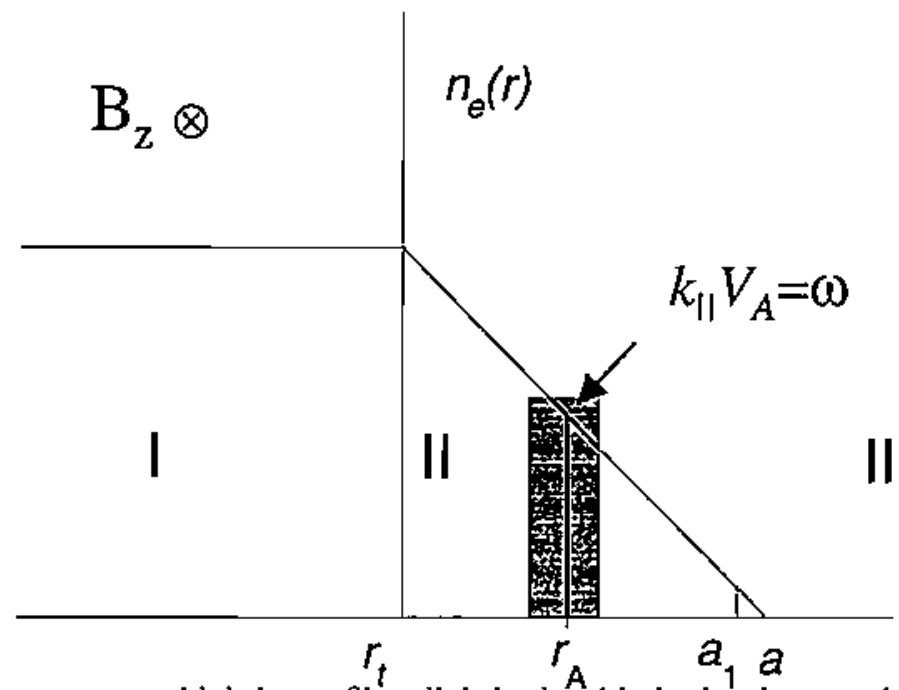

Figura 2.3 - Esquema trapezoidal do perfil radial de densidade do plasma. Até a posiçāo $r=r_{t}$ a densidade é constante, passando a variar uniformemente até $r=a_{1}$. A regiāo en cinza simboliza a regiâo em torno da ressonância de Alfvén $\left(r=r_{A}\right)$, em que a aproximação magnetohidrodinâmica nāo é válida.

É feito um desenvolvimento de Taylor dos coeficientes inomogêneos das equaçōes de Maxwell em torno de $r=r_{A}$, supondo-se $L_{r} \gg c_{s} / \omega_{c i} ;\left|r-r_{A}\right| \ll r_{A}$. Entāo, usando a relação aproximada entre as componentes $E_{r}, E_{\theta}$ e $E_{z}$ (importante perceber que aqui, diferente das Eqs.30a-c, também extraídas das Equaçōes de Maxwell, as derivadas radiais não são nulas),

$$
\begin{aligned}
& {\left[m^{2}+r^{2}\left(k_{z}^{2}-\varepsilon_{11} \frac{\omega^{2}}{c^{2}}\right)\right] E_{r}=-\mathrm{i} \frac{d}{d r}\left[\frac{m}{r} E_{0}+k_{z} E_{z}\right],} \\
& E_{z} \approx \mathrm{i} \frac{k_{z} c^{2}}{\varepsilon_{33} \omega^{2}} \frac{\partial}{\partial r}\left(E_{r}\right)
\end{aligned}
$$

a equação para a componente $E_{r}$ dos campos de RF pode ser obtida:

$$
\frac{d}{d x}\left[\left(\frac{\varepsilon_{1 \mathrm{i}, A}}{\varepsilon_{33, A}}\right) \frac{d^{2} E_{\mathrm{r}}}{d x^{2}}-k_{z}^{2} L_{r}^{2} \frac{x}{x_{a}} E_{\mathrm{r}}\right] \approx 0,
$$

onde $\varepsilon_{j j, A}=\left.\varepsilon_{j j}\right|_{r=r_{A}}, x_{a}=\left(a_{1}-r_{A}\right) / L_{r}$, é é introduzida a variável $x=\left(r-r_{A}\right) / L_{r}$.

A soluçāo MHD ideal formal, com AWR local, $E_{r} \sim 1 / x$, pode ser facilmente obtida da Eq.(46) no limite de "plasma de condutividade infinita" $\left(\varepsilon_{33} \gg \varepsilon_{11}\right.$, pois com condutividade infinita não há campo elétrico paralelo). Por outro lado, o limite MHD correto 
deve ser tomado da soluçäo completa da Eq. (46) por causa do problema de contorno. Integrando a Eq.(46) uma vez em $x$, a equaçăo de Airy inomogènea na variável adimensional 7 pode ser obtida:

$$
\ddot{\mathcal{E}}-\tau \mathcal{E}=F_{T}^{-1}, \quad \tau=g^{1 / 3} x, \quad g=\left(\frac{k_{s}^{2} L_{r}^{2}}{x_{\alpha}} \frac{E_{3, A}}{\varepsilon_{11, A}}\right) .
$$

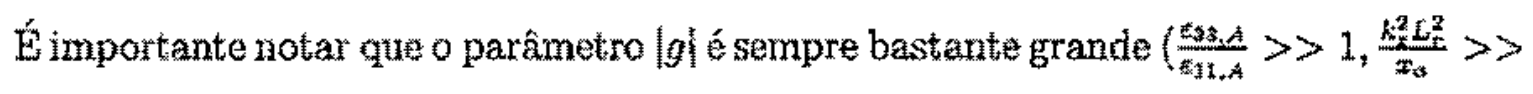
1). Entäo, a soluçäo geral da Eqa.(46) pode ser descrita via funçóes de Airy ${ }^{223}$ como

$$
E_{r}=A_{1} A(r)+B_{1} B i(r)+C_{1}(\tau)
$$

onde $A_{1}, B_{1}, B_{1}$ säo coeficientes arbitrános e $\mathcal{E}$ é uma das duas soluçoes $[H(\tau), G i(r)]$ da equaçāo inomogênea de Airy ${ }^{(23) \mid, ~ E s t a s ~ d u a s ~ s o l u c ̧ o ̄ e s ~ s a ̃ o ~ n o m a l i z a d a s ~ p e l a ~ r e l a c ̧ a ̃ o ~}$ $G(\tau)+H i(\tau)=B(\tau)$. Se dissipação da onda näo é levada am conta, o comportamento assintöhico destas funçōes na variável real, $\tau \rightarrow \pm \infty$, pode ser facilmente encontradd 23 ! A funçāo $G$ cimita lima

$$
G i(\tau) \sim \frac{1}{\pi \tau^{2}}, G i(-\tau) \sim \frac{-1}{\pi \tau}+\pi^{-1 / 2} \tau^{-1 / 4} \cos \left(\frac{2}{3} \tau^{3 / 2}+\frac{\pi}{4}\right)
$$

e a função Ha $(\tau)$ é ilimitada quando $r \rightarrow-\infty$. No caso de $|g| \gg 1$, a soluçăo da Eq.(47) e:

$$
E_{r}=A_{1} A(t)+C_{1} G i(r)
$$

onde o efeito de conversão de modos é representado pela funçäo $G i$ e $A i(\tau)$ é a autofunção dos modos.

No caso de plasmas "quentes" (velocidades de fase $w / k_{\|} \ll v_{T}$ ), a KAW(24) aparece na regiano de major densidade do plasma, $x<0$. No caso de plasmas "arios" (velocidades de fase $\left.\omega / k_{4} \gg t_{r}\right)$, a soluçăo oscilatória relativa à $S Q E A W(18,20,22$ aparece na região do plasma entre o ponto de conversão e a borda da coluna de plasma, $r_{A}<r<a$. Aqui, 
dois tipos de ondas aparecem. Uma delas é excitada pela componente paralela do campo elétrico no vácuo e autra gerada via efeito de conversão de modo. Nota-se que na Eq. (50) (quando $\left.\varepsilon_{33} \rightarrow \infty\right)$ não há limite parà a soluçăo MHD ideal $(\sim 1 / \tau)$, como discutido na Ref. [22],

Cusando a soluça da Eq. (50) com a solução para a regiầo de plasma homogênê, $E_{r} \approx \tau^{|m|-1}$, obthêm-se a relaçăo entre os coeficientes, $C_{1} / A_{1} \approx\left[T A t^{t}\right]_{r=T_{i}}$, onde $T_{i}=$ $g^{1 / 3}\left(r-r_{A}\right) / L_{r}$. Finalmente, a condicäo de ressonancia (ver, por exemplo, a Ref. [18]) para a excilaçắa da SQEAW pode ser escrita como:

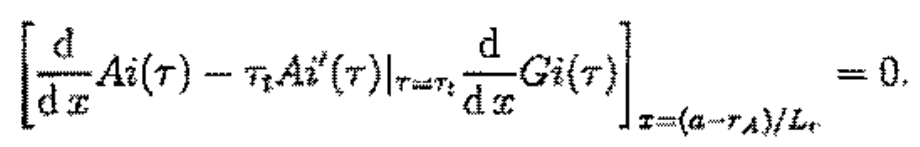

A soluçăo dada pela Ea. (50) é dramaticamente modificada se levado em consideraçäo o efeito de díssipaça da onda, que se relaciona com a parte imaginária do tensor dielétrico, Ime $E_{3}$. Neste caso, para a solução particular da $\mathrm{Eq},(47)$, a funçēo $\pi^{-1} e_{0}(-\tau)$ pode ser escolhida, onde a função generalizada de Airy.

$$
e_{0}(-\tau)=\int_{0}^{\infty} \exp \left(\tau t-\frac{t^{3}}{3}\right) d t
$$

é tomada da Ref. [25]. Esta funçāo é limitada através do eixo imaginário, decresce assintoticumente com $\sim 1 / \tau$ (quando $|\tau| \rightarrow \infty,-2 \pi / 3<\arg T<2 \pi / 3$ ) e esta soluçäo particular é unica. Na Eq.(47), o valor de g é complexo porque a componente complexa tensor dielétrico, ${ }_{33 a}$, a raiz de $g^{1 / 3}$ devem ser tais que o argumento, $\arg \tau=\arg g^{1 / 3}=$ $\alpha$ pertence ao setor duplo $\pi / 3<|\alpha|<2 \pi / 3$. As soluçoes $h_{1}(r), h_{2}(\tau)$ da equaçäo homogênea de Airy podem ser descritas tomo uma combinaça das funçôs de Airy, $h_{1_{2}}(-T)=B i(-\tau) \pm \mathrm{i} A z(-T)$, porque estas combinaçoes produzem funcões que crescem exponencialmente uas duas direcöes, Para as condiçoes do plasma "frio", $\omega / h_{1} \gg$ the $_{T \text {, }}$ os 
comportamentos assintóticos destas funçôes 23$\}$ são:

$$
\begin{aligned}
& h_{1,2}(-\tau) \sim \frac{1}{2 \pi^{1 / 2}} \tau^{-1 / 4} \exp \pm\left(\frac{2}{3}|\tau|^{3 / 2} \sin \frac{\arg g}{2}-1 \cos \frac{\arg g}{2}+\frac{\pi}{4}\right), x>0 \\
& h_{1,2}(-\tau) \sim \frac{1}{2 \pi^{1 / 2}} \tau^{-1 / 4} \exp \mp\left(\frac{2}{3} \mid t^{3 / 2} \cos \frac{\arg g}{2}-i \sin \frac{\arg g}{2}+\frac{\pi}{4}\right), x<0
\end{aligned}
$$

Finalmente, quando $\left|g^{1 / 3}\right| \gg 1$ e $x$ está no intervalo $\left(r_{t}-r_{A}\right) / L_{\mathrm{r}}<x<\left(a-r_{A}\right) / L_{r}$ a soluçäo da Eq. (47) será:

$$
E_{*}=A_{1} h_{1}(-\tau)+C_{1} \pi^{-1} e_{0}(-r)
$$

Na tquaçāo acima, a solução da eq̣uaģăo homogênea de Airy, $h_{1}(-r)$, representa a parte de camada dissipativa do campo de RF na perifería do plasma, enquanto a soluçấo eo $(-T)$ representa a parte de conversão de modos do campo de RF no ponto da AWR local, $r=\tau_{A}$ A condiçáco para a prestença da AWR local é:

$$
\frac{2}{3}\left(\frac{0-r_{A}}{L_{\tau}}\right)^{3 / 2} \ln g^{1 / 2} \gg 1
$$

Usando este limite a supondo um afeito de dissipaçāo fraco, Im $y<$ Reg, obtém-se uma soluçăo que é parecida com a soluçăo para MHD ideal, $E_{r} \sim g^{-1 / 3} x^{-1}$, A meialargura da ressonância onde a dissipação é forte, $\delta r$, é da ordem de $g^{-1 / 3} L_{\mathrm{r}}$

Importante notar que no caso de um perfil de densidade arbitrário as condiçốc para o limite de MHD ideal podem ser escritas como:

$$
\begin{aligned}
& \int_{r_{A}}^{a}\left[\operatorname{Im} k_{r} \mid d r \gg 1, \quad w / k_{\|} \gg v_{p}\right.
\end{aligned}
$$

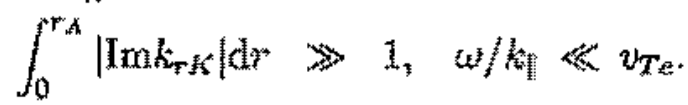

onde $k_{r K}$ é obtido da $\mathrm{Eq},(43)$.

Integrando a Eq(45) e usando a propriedade da função $e_{0}^{[25]}$ no plano complexo,

$$
\int_{0}^{(\cos \alpha+i \sin \alpha) \cdot \omega} \operatorname{Re} e_{0}(\tau) \mathrm{d} \tau=\frac{\pi}{2}
$$


obtém-se o comportamento da componente $E_{\theta}$ no limite MHJi 17,26 .

$$
\begin{aligned}
& E_{t e} \sim 1+\frac{r_{A}-r_{i}}{r_{A}} \log \left|\frac{r_{A}-r_{A}}{r_{A}-r_{t}}\right|, \quad \text { para } x>0 \\
& E_{0} \sim 1+\frac{r_{A}-r_{t}}{r_{A}}\left(\log \left|\frac{r_{A}-r_{0}}{r_{A}-r_{i}}\right|+i \pi\right), \text { para } a<0
\end{aligned}
$$

Usando a expressäo assintótica pode-se facilmente obter o valor da potência dissipadat 20 na ressonâncita do modo de Alfển local através do teorema de Poynting (ver Apendice D):

$$
\operatorname{Re} \frac{1}{2} \int_{V} J^{*} \cdot \vec{E} d u=\frac{L_{y} L_{z} e^{2}}{8 \pi \mu_{0} \omega\left(k_{z}^{2}+k_{y}^{2}\right)}\left|E_{y}^{0}\right|^{2} \pi \frac{r_{A}-r_{t}}{r_{A}}
$$

\subsection{Análise do Efeito das Impurezas}

No processo de aquecimento de plasmas em tokamak com ondas de Alfvến, inpurezas pesadas e parcialmente ionizadas, como oxigenio ou ferro, introduzem zones de ressonância ciclotrônica iônica que podem modificar as características de dispersão destas ondas. Um dos assuntos importantes na pesquisa de fusão termonuelear controlada diz respeito aos efeitos de impurezas mais leves, como deutério, hêlio ou carbono, nas propriedades espectrais e dispersivas das ondas de Alfuén. Quando a frequência da onda de Alfvén for inferior à frequênela ciclotrônica iônica tanto da espécie principal do plasma quanto das impurezas completamente ionitzadas, só acontecerâo mudangas pequenas nas

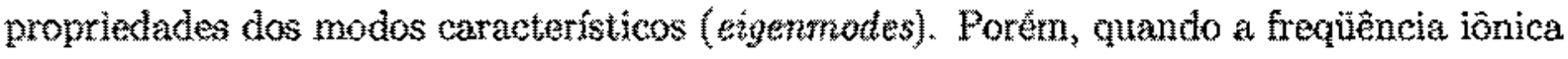
ciclotrônica das impurezas leves forem comparáveis à trequîencia da onda de Alfén, modifieaçöes significativas da propagaçăo do modo de Alfvén e das propriedades de aquecimento sấo previstes.

Vatrias experiências, como as realizadas no tokamak TCA [9], produziram aquecimento eficiente do plasma com o modo $G A$. O modo $G A$ tem uma frequêencia, $w_{C A}$ próxima à frequiência mínima do contínuo de Alfyén $w_{s h}(r)$, definida numa generalização 
aq $\mathrm{Eq} \cdot(5)$ como

$$
\omega_{s h(x)}=\ln (r) c_{A}(r)
$$

$\mathrm{Na} \mathrm{Eq}(50), c_{A}(r)$ è a velocidade local de Afrvén,

$$
\frac{1}{c_{A}^{2}(r)}=\frac{1}{c^{3}} \sum_{i}\left(\frac{\omega_{p, i}}{\omega_{c, i}}\right)^{2}
$$

onde $\omega_{p i}$ e $\omega_{c:}$ sầ, respectivamente, as frequências locais de plasma do íon e ciclotrônica łônica, $c$ é a velocidade de luz, $h_{i}(r)=[n+m / q(r)] / R_{0}$ é o número de onda paralelo, $q(r)=r B_{t} / R_{0} B_{\theta}$ é o fator de segurança (ou transformada rokacional inversa) do tokamak, e r e $R_{0}$ são, respectivamente, a distância do eixo magnético (ou raio menor) e distiancia ao eixo principal (ou raio major) do tokamak. Descobriu-se que a potência de RF no modo GA é eficientemente dissipada na regiano central do plasma pelo amortecimento eletronico de Landau ou possivelmente pelo amortecimento colisional. Um aquecimento eficiente tambern foi observado quando o perfil da densidade de corrente era plano. Quando o perfil de corrente é plano, o modo GA aparece dentro do Contínuo de Alvéntol.

Para o propósito de analisar teoricamente o comportamento de ondas de Alfvén Torsionais e ondas de Alfvén Globaís e processos de aquecimento por RF; os modelos

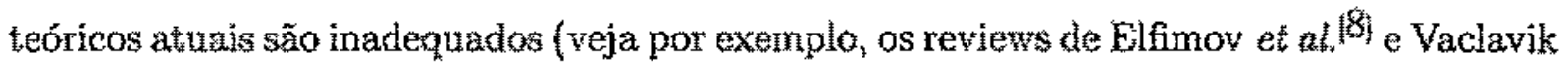
e Appert 11 ). Modelos antigos foram em grande parte baseados ern plasmas com uma única espécie de ion. Os efeitos de mủitiplas espécles de íon no espectro de Alfén nä́o receberam muita atençä̃o. Appert et al. 271 e Li et al ${ }^{281}$ estudaram numericamente os efeitos de impurezas quando a ressonância ciclotrônica da impureza está localizada perto do centro do plasma. Porém, sob esta condiçäo, a onda Global de Alfvén não é excitada. Nesta tese, a teoria das ondas de Alfuén en plasmas de tokamak é estendida para incluir os efeitos de impurezas, quando as ressonâncias ciclotrónica ionica principal e fon-ion-hibridas nâo estāo presentes na seção transyersal do plasma. É mostrado em 
particular que as impurezas podem estender a faixa de númẹros de onda e densidade acirna das quais ondas Globais de Alvẻn (e semelhantes) podem ser excitadas tanto dentro como tora do Continuo de Alvén (com o aparecimento destes no limiar do contínu ionion-híbrido de Alfvén). Os resultados teóricos também demonstram a importânciza do modo global na faixa do contínuo de freqüencias. No contínuo, o modo global tern uma largura espectral mutto maior, significando que as condiçöes de ressonância seräo mais provavelmente satisfeitas durante a descarga do tokamak. Este resultado é importante, porque provê um método para excitar a onda Global de Alfvển em um plasma de tokamak com impurezas ionizadas.

\subsubsection{Modelo Cilindrico de Plasma e Resultados dos Cáleulos}

A análise numérica está baseada no cơdigo unidimensional cinético de Dmitrieva et al ${ }^{[15]}$, chamado EPCY, que calcula a dispersão e dissipaçăo das ondas de Alfvén em uma configuraça de plasma cilíndrico com duas espécies. O modelo cilíndrico no qual o código é baseado corresponde à aproximaţăo de grande razäo de aspecto $(R / a>>1)$ para

tokamaks. O código EPCY, de forma semelhante a outros codigos relacionados $11,28,20$, resolve o conjunto linearizado de equações de Maxwell-Vlasov, postas como um problema de contorno, para os campos das ondas de RF produzidos por uma antena externa em uma coluna de plasma magnetizada. O plasma 6 um cllindro de raio a. Em torno deste há uma regiäo de vácuo envolta por uma parede condutora de raio $c$. Na regiăo de vảcuo, há uma antena com corrente superficial localizada em uma superfície cilindrica de raio b. As componentes $z$ e $\theta$ da corrente superficial podem ser representadas pela série de Fourier,

$$
J_{z_{1} \theta}=\sum_{m, n} J_{z, \theta}^{(z n, n)} \delta(r-b) \exp \left[\left(m(m)+n z / R_{0}-w t\right)\right]
$$


onde $y_{z, \theta}^{(m, n)}$ é a amplitude constante do harmônico $(m, n)$, u é a frequência do gerador. Nesta tese, é utilizado um modelo com um único valor para os números $m, n$, modelando uma antena helicoidal ${ }^{8}$ na Eq.(57). A Eq.(57) também pode servir como urn modelo de um sisterna de antena de multi-modo (tipo Nagoya-III).

O código EPCY está baseado na aproximagão que p. p pa săo ambos muito menores que $L_{r}$ e $\lambda_{r}$, onde $L_{r}$ é o comprimento característico da inomogeneidade radial do plasma e $\lambda_{r}$ é o comprimento característico da variação radial do cumpo de RF. Nesta análise não sāo levados em conta o efeito do xaio de Larmor iônico finito na dispersão e dissipaçäo da onda de Alfén Torsional e da onda Global de Alfuén, porque a dissipação destas ondas, como também a conversäo de modo no contínuo de Alfvén, näo depende do raio de Larmot Iônico[8, 11]. Além disso, sob as condiçōes que säo caracteristicas de tokamak métios cono o TCABR, a inércia eletrớnica tem um efeito mais forte na dispersão da onda de Alfvén cinética que a temperatura iônica. Assim, desprezando efeitos relacionados com $\rho_{e}$ $\rho_{i}$ finitos, o tensor dieletrico, $t$, reduz se a sua forma local, considerando o amortecimento de Landau eletrônico. Fspecificamente, $\epsilon$ reduz-se a seguinte tensor,

$$
\epsilon=\left(\begin{array}{ccc}
\epsilon_{11} & \epsilon_{12} & 0 \\
\epsilon_{21} & \epsilon_{22} & 0 \\
0 & 0 & \epsilon_{33}
\end{array}\right) \text {, }
$$

onde as componentes näo nulas săo,

$$
\begin{aligned}
& \epsilon_{1 !}=\epsilon_{22}=1+\frac{\omega_{p i}^{2}}{\omega_{c i}^{2}-w^{2}}+\frac{i w_{z z}^{2}}{\omega_{c z}^{2}-w^{2}} \\
& \epsilon_{12}=-\epsilon_{21}=i\left[\frac{\omega \omega_{p i}^{2}}{\omega_{c i}\left(\omega_{c i}^{2}-\omega^{2}\right)}+\frac{\omega \omega_{p Z}^{2}}{\omega_{c Z}\left(\omega_{c Z}^{2}-\omega^{2}\right)}-\frac{\chi^{h} c_{c}^{2}}{\omega^{2}}\right]
\end{aligned}
$$

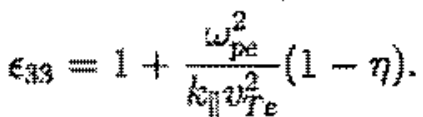

Na Eq. (59), diferente da expresäo mostrada na seção 2.2 , esta induído o termo de 
cisalhamento do campo magnético, na quantidade $\chi$, que tem a seguinte definiz̧ä:

$$
x=r \frac{B_{z}^{2}}{B^{2}} \frac{\partial}{\partial r}\left(\frac{B_{Q}}{r B_{2}}\right)
$$

enquanto na $\mathrm{Eq} .(60)$, $n$ (jâ mostrada na seçâo 2.4) é una integral complexa que responde pelo amortecimento de Landau.

0 indices $(1,2,3$,$) se referem, respectivamente, às componentes radial (r)$, binormal (b), e paraleh (ll) do tensor dielétrico com respeito as linhas de campo magnético de equilíbrio.

A introduçäo do termo colisional de Krook (ver parágrafo após a Eq.(4d)) permite reduzir o numero de pontos de discretiza

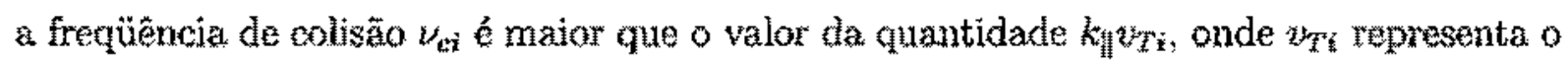
velocidade térmica tởnica. Alérn disso, a freqựencia te colisăo suposta nos cálculos esta na faixa entre $0,01 \omega$ e $0,04 \omega$ ( $\omega$ é a frequência do gerador), o que é consistente com a trequiência de coliaäo de elétron-ion no plasma do TCABR.

O perfil de corrente axial, $f(r)$, é suposto ter a forma $j(r)=j 0\left(1-r^{2} / a^{2}\right)^{\mu_{3}}$, onde $n_{j}$ $=\left(q_{a} / q_{0}-1\right)$, com $q_{a} / q_{0}$ sendo a transfoumada rotacional inversa (ou fator de seguranca) na interface plasma-vácuo nornalizada a seu valor ao centro da coluna de plasma. A densidade eletrônica do plasma é representada pela expressäo,

$$
n_{e}=n_{a}\left[1-\left(1-\frac{n_{a}}{n_{a}}\right) \frac{r^{2}}{a^{2}}\right]^{a_{k}}
$$

onde $n_{0}$ e $n_{z}$ säo, respectivamente, as densidades eletrônicas central e perifética do plasma, e $\mu_{\mathrm{n}}$ é o parấmetro variando de 0,6 a 3 (em geral, aquí e usado o valor 1), que governa o perfil de densidade. Säo supostos perfis de densidades e tempextutura semelhantes para impurazas. Na periferia da coluna de plasma, a densidade tem uma deseontimudade que E introduzida para evitar modos superficiais.

Usando o Código $E P C Y$, pode-se calcular a dependência da parte real da impedância 
de carga da antena como uma funçüo da freqüteneia do gerador. A geometria e os parâmetros do plasma supostos säo característicos do tokamak TCABR: $a=18 c m_{1}$ $b=18,5 \mathrm{~cm}_{2} d=23 \mathrm{~cm}$, e $R_{0}=61 \mathrm{~cm}$, correspondendo a uma razäo de aspecto de 3,4. Para o plasma, é suposto que as temperaturas eletrönica e iônica säo, respectiva* mente, $T_{e}=500 \mathrm{eV}$ e $T_{i}=300 \mathrm{eV}$, enquanto a densidade máxima dos elétrons, $n_{0}$, varia entre $1,0 \times 10^{13}$ e $5 \times 10^{13} \mathrm{~cm}^{-3}$. A densidade de fons foi escolhida de forma que a condigäo de neutralidade de carga seja satisfaita, $Z n_{Z}+n_{H}=n_{e}$, onde $n_{z}$ e $n_{H}$ designam, respectivamente, as densidades ionicas da impureza e do hidrogennio. Finalmente, o campo magnético toroidal, $B_{i}$, varia entre 10 e $15 \mathrm{k} G$, supondo-se $q=1,01$ no cixo magnético, Q variando entre 3 e 4

Na Fig. 2.4, é apresentado um gráfico da parte resistiva da impedănoia $\left(Z_{A}=W / J_{A}^{2}\right.$ ) da antena helicoidal contra a frequîencia para um plasma de hidrogênio, com adiçăo de carbono totalmente ionizado, numa proporçăo relativa à densidade total $\left(n_{c} / n_{0}\right)$ que é variada até $3 \%$. A frequência f é medida em MHz. Os parâmetros seguintes foram usados na Fig. 2.4: $B_{t}=10 \mathrm{kO}, n_{0}=3 \times 10^{13} \mathrm{~cm}^{-3} ; \mu_{n}=1, \mathrm{e} \mu_{z}=2,01$. Além disso, supõe-se que só um modo esteja presente, isto 6,0 modo $m=-1$, $m=-4$. Os fortes picos presentes nos gráficos de impedância correspondem aos modos característicos GA. Estes modos ficam situados dentro do contínuo inferior de Alfvến, sugerindo um aquecimento central do plasma, por conversão de modo em modos cinéticos de Alfvén. Considerando que a ressonäncia é larga, pequenas mudanças na freqiiência dos modos GA,

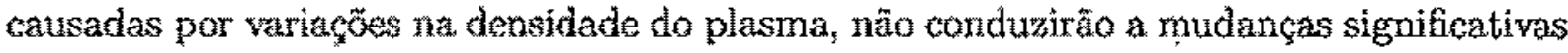
na impedancia ta antena. Para plasmas puros, ondas de Alfén Globais (descobertas por Ross et al ${ }^{20}$ e estudadas por Appert et al ${ }^{29}$ para $\left.m=-1, n=-2\right)$ são fortemente excitadas no contínuo das ondas de Alfvén e só existem para números de onda toroidais e poloidais negativos $(m, n<0)$. 


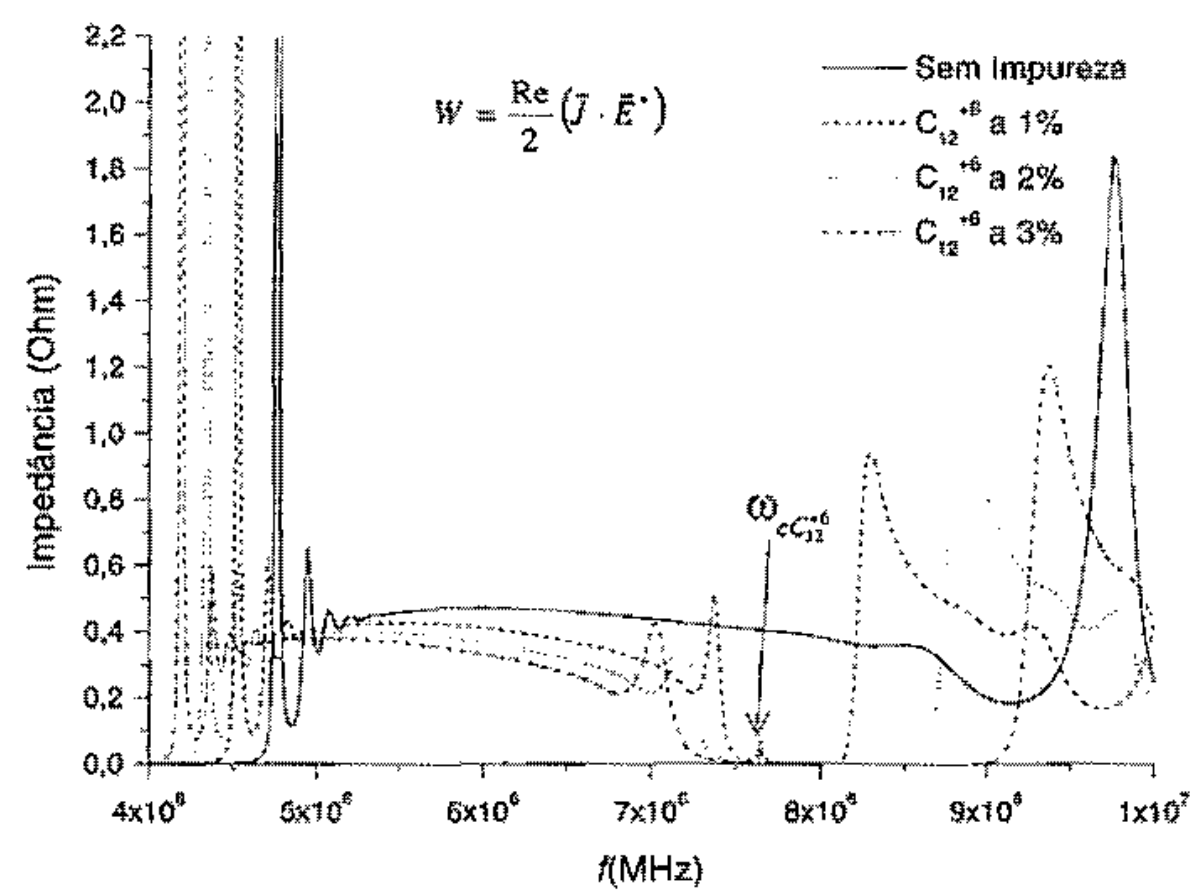

Fig.2,4. Impedância da antena contra frequiência em $M H_{z}$ para um plasma de hỉdrogenio com concentraçöss de impureza de carbono diferentes. As carvas de impedàntia (linhas sỏlda, tracejada,

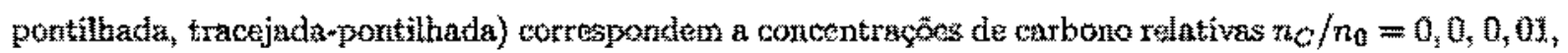
$0,02,0,03$, respectivamente. Uma configuração da antena de modo com número de onda poloidal $m=-1$ e número onda toroidal $n=-4$ é escolhida. Os parâmetros de plasman săo $B_{z}=10 k G, q_{0}=1,1, q_{a}=3,3$,

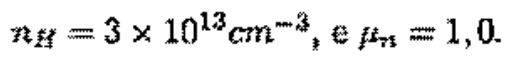

Com uma pequena populaçäo de impurezas acrescentada ao plasma, lacunas aparecem no contínto de Alfvén próximo à ressonância ciclotrônica lônica da impureza, $\Omega \approx$ $\omega_{c}^{*}=7,65 \mathrm{MHz}$ Isto pode ser entendido considerando a relação de dispersão local que governa o contínuo de Alevễn,

$$
w^{2} \epsilon_{11}(r)=k_{\|}^{2}(r) c^{2}
$$

onde $\epsilon_{11}(r)$, a componente transversal do tensor de permeabilidade, está definida pela Eq.(59). O contínuo de Alfvến e definido através das soluçes da Eq. (61) para a freqüencia como uma funçăo do raio, $\omega^{2}=\omega_{s h}(r)$. Quando a frequêencia está perto da frequiencia 
ciclotrônica iônica de uma impureza, a Aa. (61) năo tem nenhuma soluçăo real, implicando na presença de uma lacuna no contínuo.

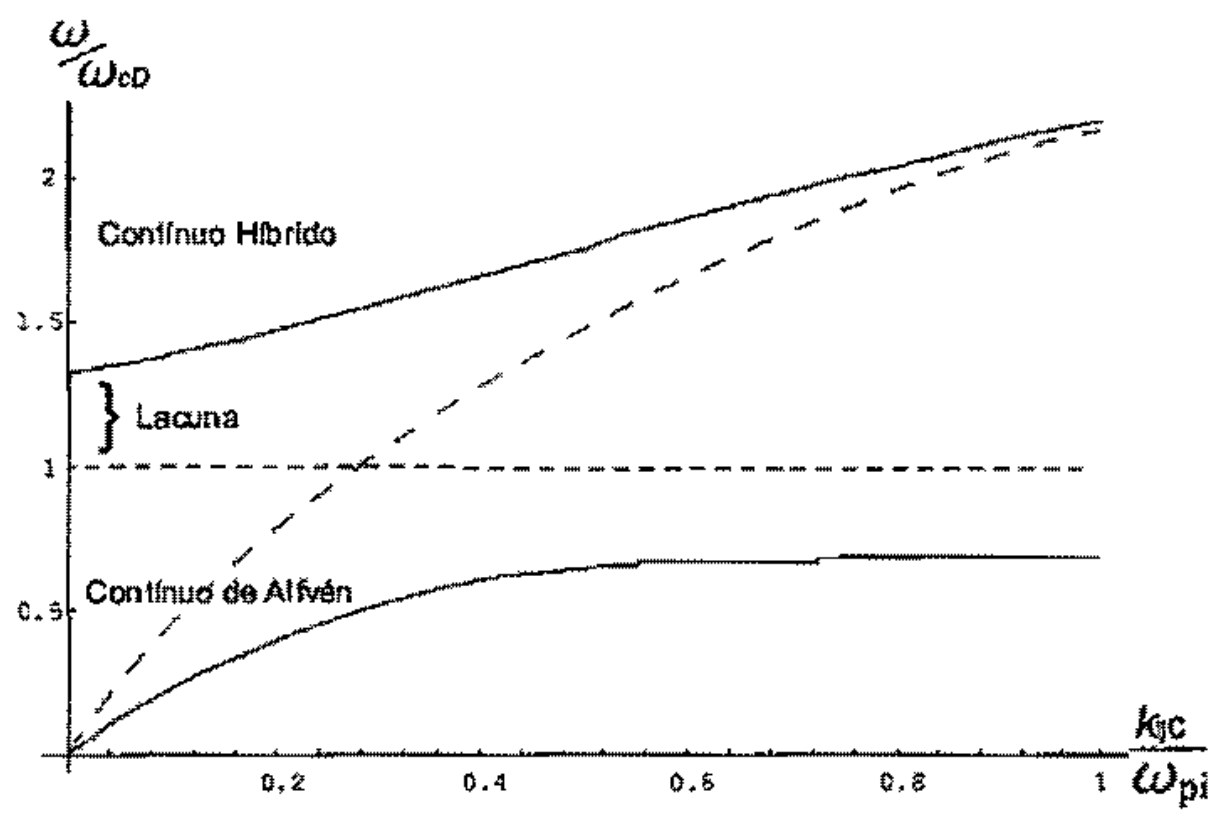

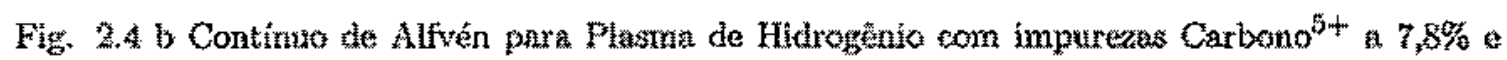
Dewtério ${ }^{+1}$ a $47 \%$

A solução da Eq.(61) para a freqüiêncía do contínuo de Alfvén tem dois ramos, com $\omega_{s f i}^{+}(r)$ e $\omega_{s h}^{-}(r)$, os quais, para um plasma com luas espécies de lon, podem ser escritos da segtuinte maneira,

$$
\left(\frac{w_{s t}^{+}}{\omega_{e z}}\right)^{2}=\frac{1+\rho_{z}+k^{2}\left(1+A^{2}\right) \pm \sqrt{\left[1+\rho_{z}+\kappa^{2}\left(1+A^{2}\right)\right]^{2}-4 \kappa^{2}\left[\rho_{z}+A^{2}\left(1+\kappa^{2}\right)\right]}}{2\left(\rho_{z} / A^{2}+1+\kappa^{2}\right)}
$$

Na Fq. (62), $\rho_{z}$ significa $n_{z} M_{z} / n_{H} M_{H}$, a razäo das ciensidades de massa de impureza e de hidrogênio, $A$ representa $M_{z} e / M_{H} e_{z}$ a relaģấo de carga-massa de impureza normal-

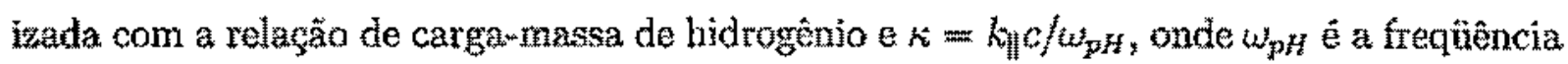
de plasma dos ions de hidrogênio. O ramo superior do contínuo de Alfwèn, $\omega_{s h}^{+}(r)$, tem um mínimo na frequência híbrida fon-1on,

$$
\left[\Omega_{t h}^{+}\right]_{\text {Tiint }}=w_{t z} \sqrt{\frac{1+\rho_{z}}{1+\rho_{z} / A^{z}}}
$$


Para modos com $m<0$ e $n<0$, menos que $25 \%$ da potencia dissipada é depositada na regiäo externa do plasma, na faixa $0.5<r / a<1$. Logo abaixo da lacuna no contínuo (ao redor 7.2MHz) há picos relacionados com os modos característicos de superfície. Na Fig. 2.4, acima do limiar do Continuo Hibrido de Alfyén, pode-se observar máximos da impedância que correspondem à onda de Superficie de Alfvén (SA) profundamente imersos no contínuo. A freqüiência desta onda SA é diminuida na direção do limiar do Contínuo Híbrido de Alfvén se a densidade de hidrogênio for reduzida de $n_{H}=3 \times 10^{13} \mathrm{~cm}^{-3}$ para $\cdots 1,3 \times 10^{13} \mathrm{~cm}^{-3}$. Finalmente, a onda Global de Alfvén surge abaixo do Contínuo Híbrido de Alvén se a densidade for menor que $n_{H} \approx 1,0 \times 10^{13} \mathrm{~cm}^{-3}$. Este efeito é ilustrado na Fig. 2.5, que mostra a impedância da antena versus freqüência, para freqüuéncias superiores às da regita da lacuna, para diferentes valores de densidade de Hidrogênio e $n_{C} / n_{0}=0,01$, constante.

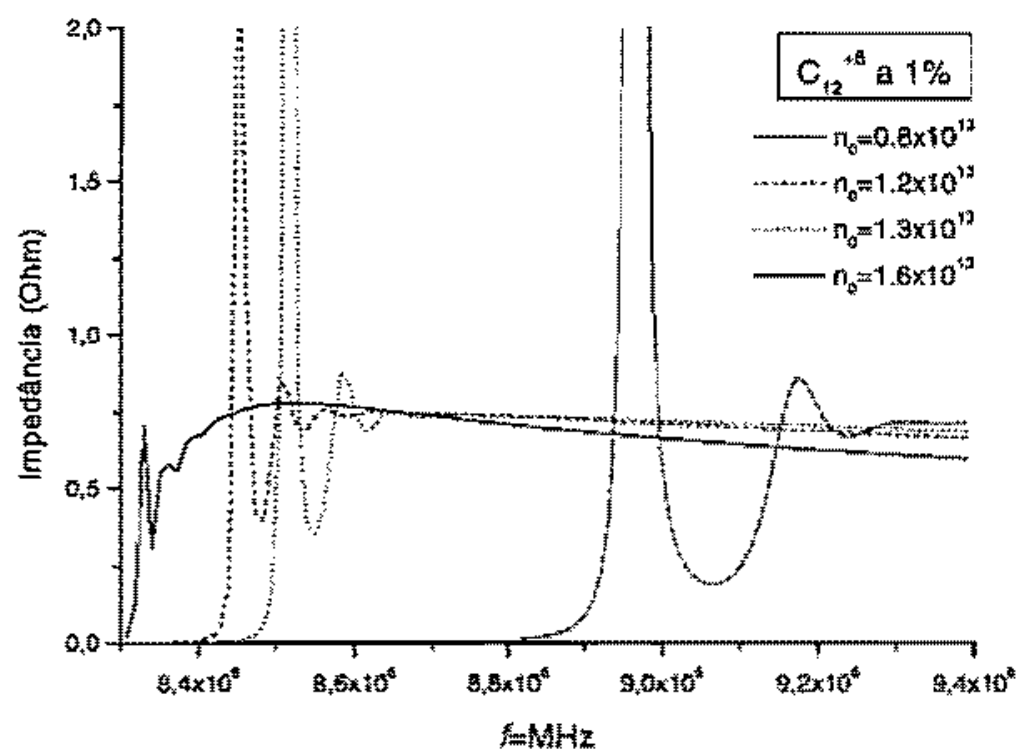

Fig, 2,5 Impedancia da antena contra frequencia para diferuntes densidades de plasma de hidrogento,

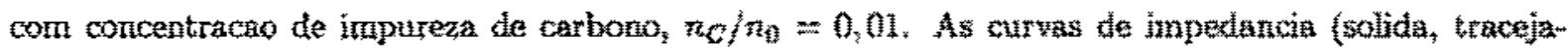

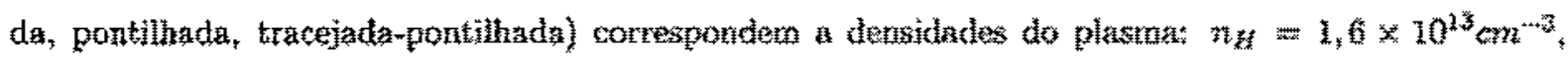

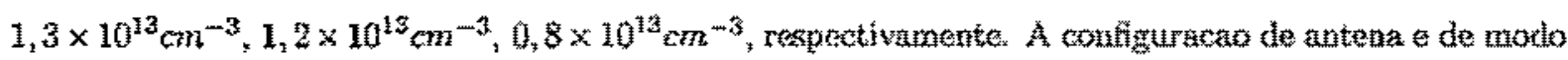
poloidal $m=-1$ e toroidal $n=-4$. Os tutros parametres do plasma sao igunds as da Fig.2,4. 
Próximo ao limiar do contínuo, a onda SA muda a estrutura dos campos da onda para um Modo Combinado de Alfvén (MCA). A estrutura dos campos de RF no MCA tem um caráter intermediário entre as ondas $\mathrm{SA}$ e ondas GA (veja 8,19$])$. Para ilustrar o caráter do $M C A_{1}$ a Fig. 2.6 a mostra a distribuiçâo radial das componentes $E_{r}$ e $E_{p}$ do campo de RF e a potência dissipada da onda, $Q$. Na Fìg. 2.6a são apresentados os resultados dos cálculos para a ressonância do MCA num plasma de hidrogênio, com densidade $n_{H}=1,2 \times 10^{13} \mathrm{~cm}^{-3}$ e concentração de impureza de carbono relativa $n_{o} / n_{i}=$ 0,01 .

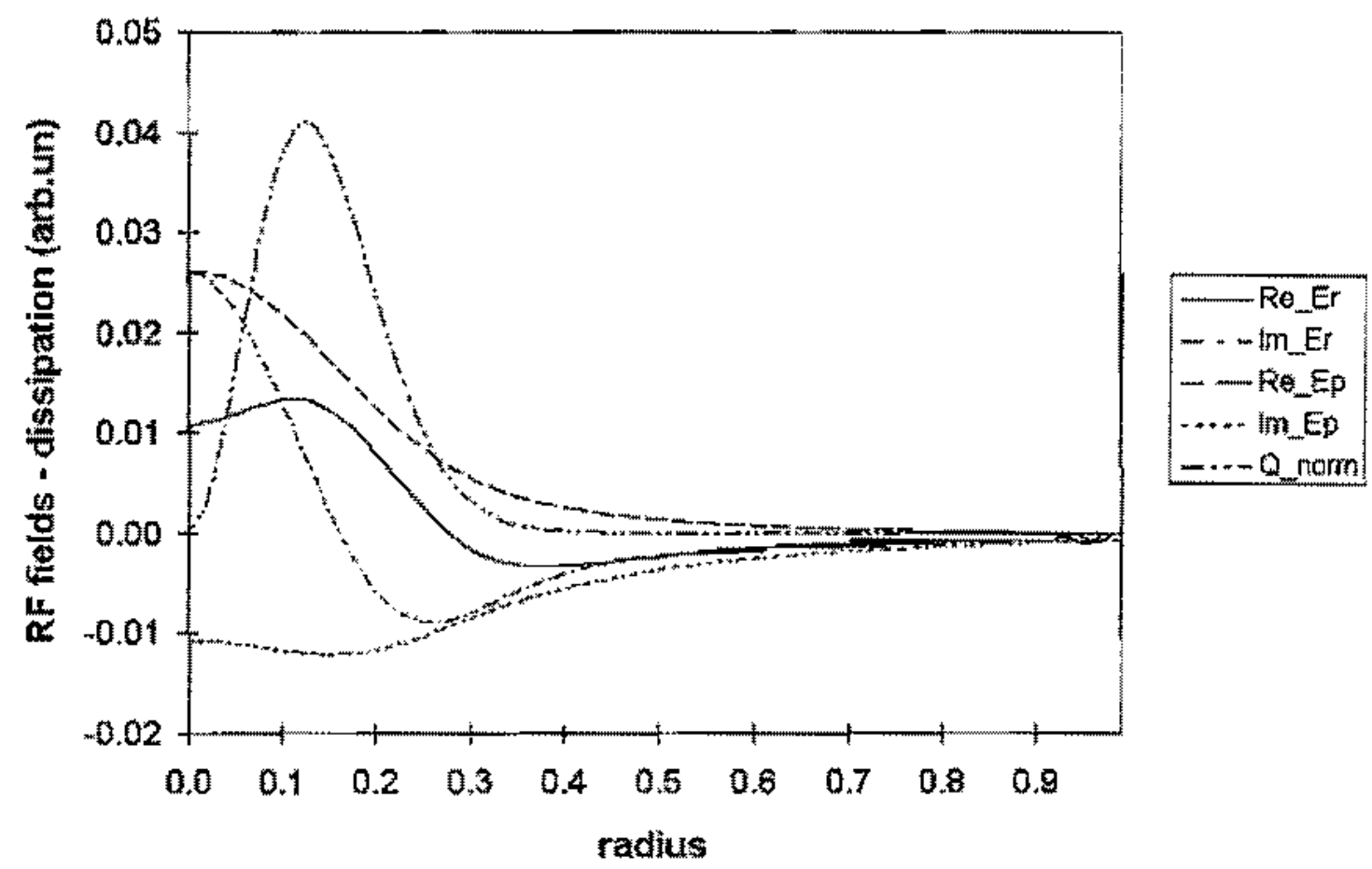

Fig, 2.6a Distribuigà radial das componentes radial, $E_{r}$, e poloidal, $E_{p}$, to campo elétrico e a potência dissipata, $Q$, da onda. As curvas (linhas sólida, tracejada, pontillhada, tracojata-pontilhada) correspondem às partes real a inaginária dis componentes do campo elétrico, ReE $E_{r}, \operatorname{Im} E_{r}, \operatorname{Re} E_{p}$ e ImE $E_{p}$, respectivanenze, am unidades arbitrárias. A linha pontillada-tracejada-pontilhada corresponde a dis-

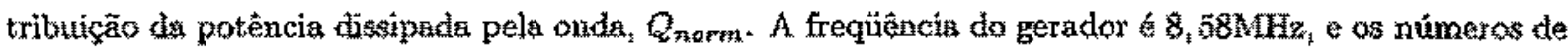
onda poloidal e toroidal säo, $m=-1$ e $n=-4$. Foram mantidos os parâmetros de plaswa indicados na Fig.2.4. 
Na Fig. 2.6a, a componente $\operatorname{Im} E_{\tau}$ do modo MCA se anula perto do centro do plasma, no raio $r=0,16$, onde o máximo de dissipação da onda é obtido. Isto significa que o MCA apresenta um ponto de conversão como a onda SA, mas aquece o centro do plasma como a onda GA. Este regime é muito bom para o aquecimento por ondas de Alfvén e geração de corrente, mas a densidade $\left(n_{H}=1,2 \times 10^{13} \mathrm{~cm}^{-3}\right)$ é muito baixa e a concentração real da impureza de carbono é muito alta para condições padrão no tokamak TCABR.

Para os números de onda poloidal e toroidal $m=-1$ e $n=-6$ e freqüência do gerador de $9,1 \mathrm{MHz}$, são encontradas as condições otimizadas para o aquecimento e geração de corrente no plasma do TCABR por ondas de Alfvén, com a densidade $n_{H}=2,3 \times$ $10^{13} \mathrm{~cm}^{-3}$ e concentração de impureza de carbono $n_{C} / n_{i}=0,02$. Os perfís de campo elétrico e potência dissipada para estes parâmetros são mostrados na Fig. 2.6b.

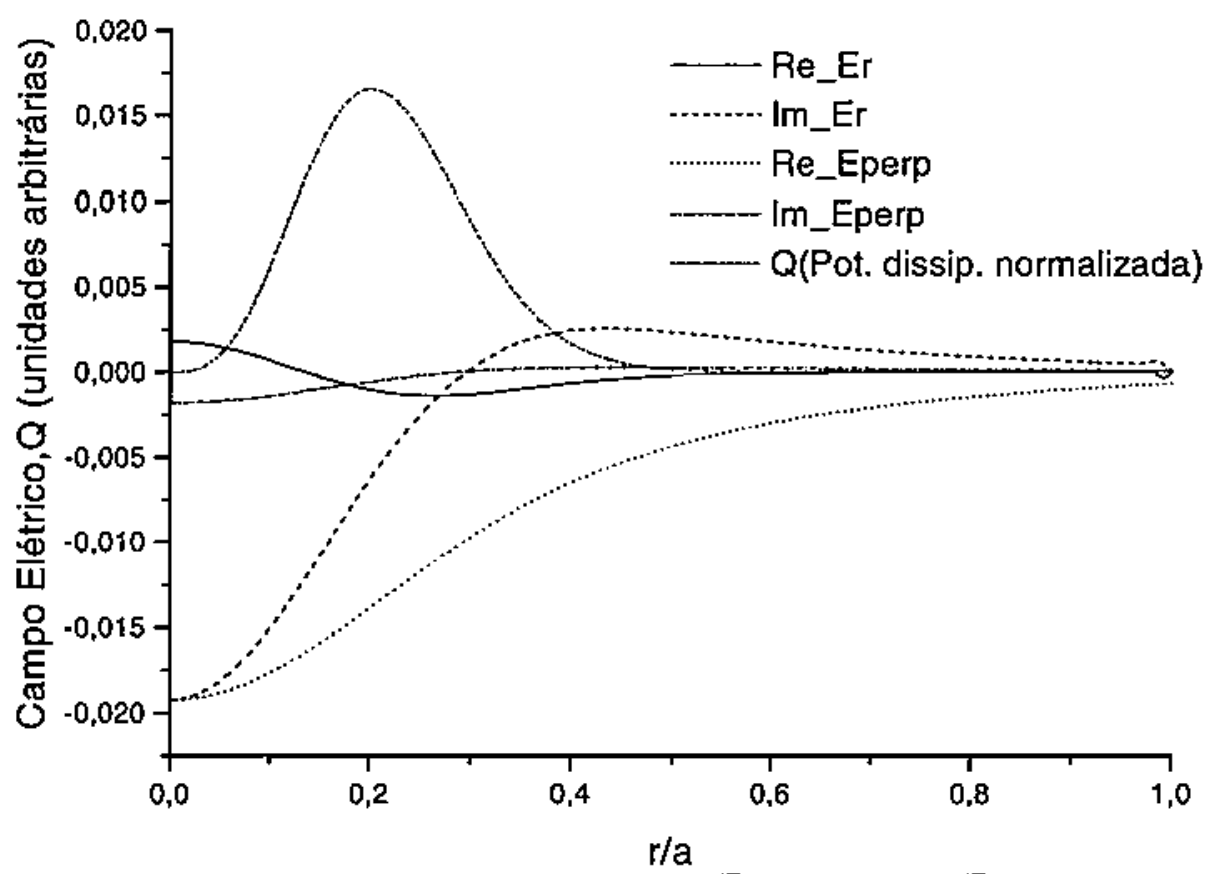

Fig. 2.6b Distribuição radial das componentes radial, $E_{r}$, e poloidal, $E_{p}$, do campo elétrico e a potência dissipada, $Q$, da onda. A freqüência do gerador é $9,1 \mathrm{MHz}$, a densidade de elétrons $n_{H}=$ $2,3 \times 10^{13} \mathrm{~cm}^{-3}$, a densidade de carbono é de $2 \%$ e os números de onda poloidal e toroidal são, $m=-1 \mathrm{e}$ $n=-6$. 
Para os números de onda poloidal e toroidal $m=-1 \mathrm{e} n=-8$ e freqüênciá do gerador de $9,72 \mathrm{MHz}$, as mesmas condiçōes otimizadas podem ser alcançadas no plasma do TCABR, com densidade $n_{H A}=3,5 \times 10^{13} \mathrm{~cm}^{-3}$ e concentraçäo de impureza de carbono $n_{C} / n_{i}=0,03$. Os cálculass mostram que a freqüência da onda GA no limiar do Contínuo Hibrido de Alfvé̉n é fixa para uma concentração de impureza fixa e para numero de onda paralelo normalizado fixo, $k_{y} c / \omega_{p H}$. Estas condiçöes de aquecimento podem ser mudadas se o perfil de densidade for modificado. A Fig. 2.7 ilustra as modificaçöes da impedância do plasma para $m=-1$ e $n=-6$ causadas pelas variaçôes do parâmetro de perfil de densidade, $\mu_{n}$

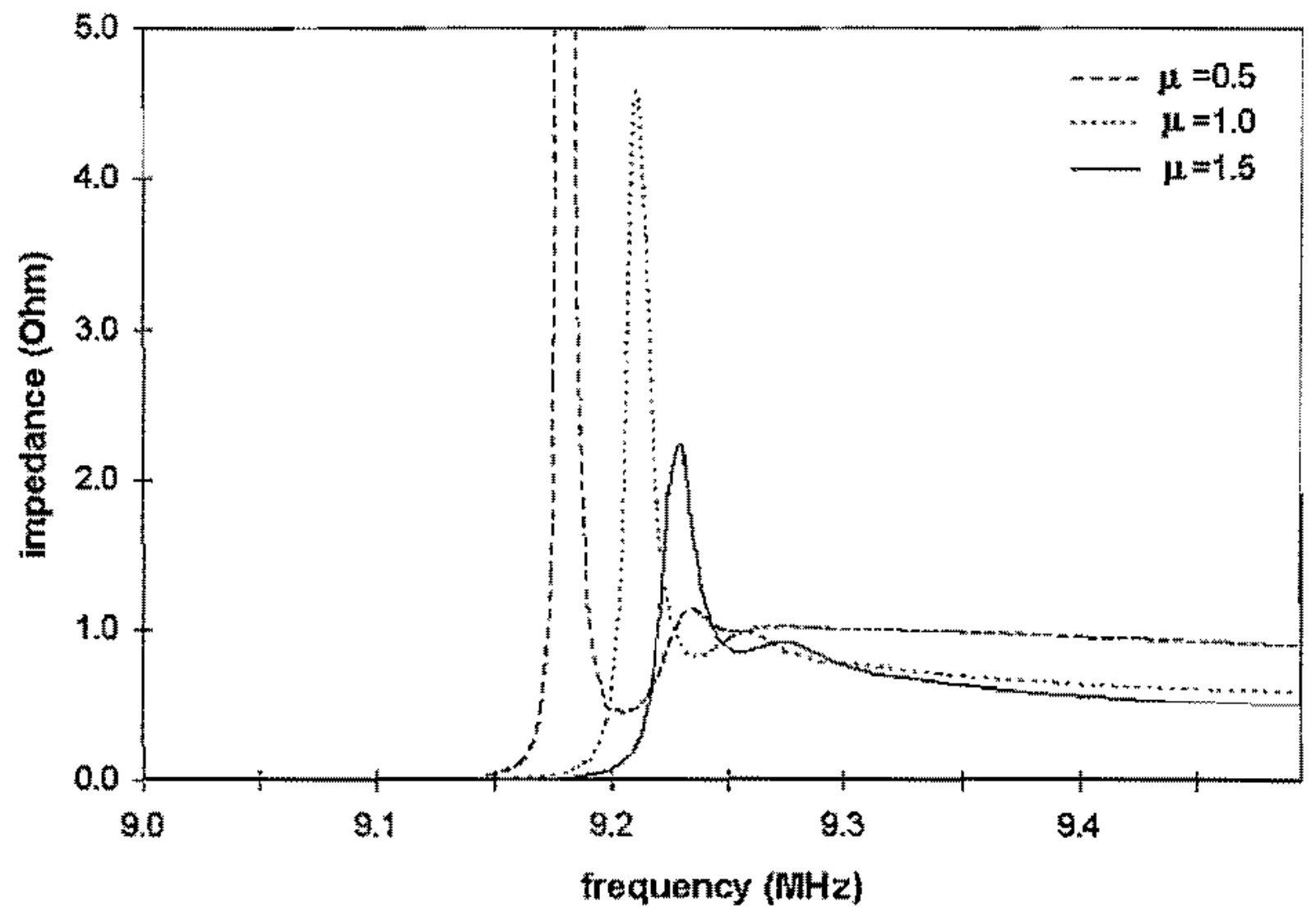

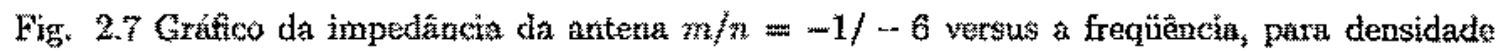

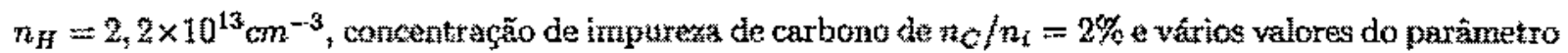
do perfil de densidade $\mu_{n}$. As curvas (linhas tracejada, pontilhada sólida) correspondem a $\mu_{n}=$ 0,$5 ; 1,0,1,5$, respectivamente. 
Na Fig. 2.7, ě mostrado que a onda Global de Alfvẻn se situa abaixo do Contínuo Hibrido de Alfvén para $n_{H}=2,2 \times 10^{13} \mathrm{~cm}^{-3}$ e $t_{n}=0,5$. A onda GA entra na contínuo quando o perfil de densidade é estreitado (para $\mu_{n}=1,5$ ). Nota-se que o comportamento da onda global, perto do Contínuo Híbrido de Alfvển, nẫo é tão sensível à variação do perfil de corrente (ou o parâmetro q) em comparação com as variaçỏes do parâmetro $\mu_{n}$.

Na Fig. 2.8, são apresentadas as caracteristicas de dispersão da onda SA e modos GA e ilustrada a lacuna no Contínuo de Alfvén, para um plasma de hidrogènio com a espécie de impureza carbono e com perfil de densidade parabólico $\mu_{n}=1,0$.

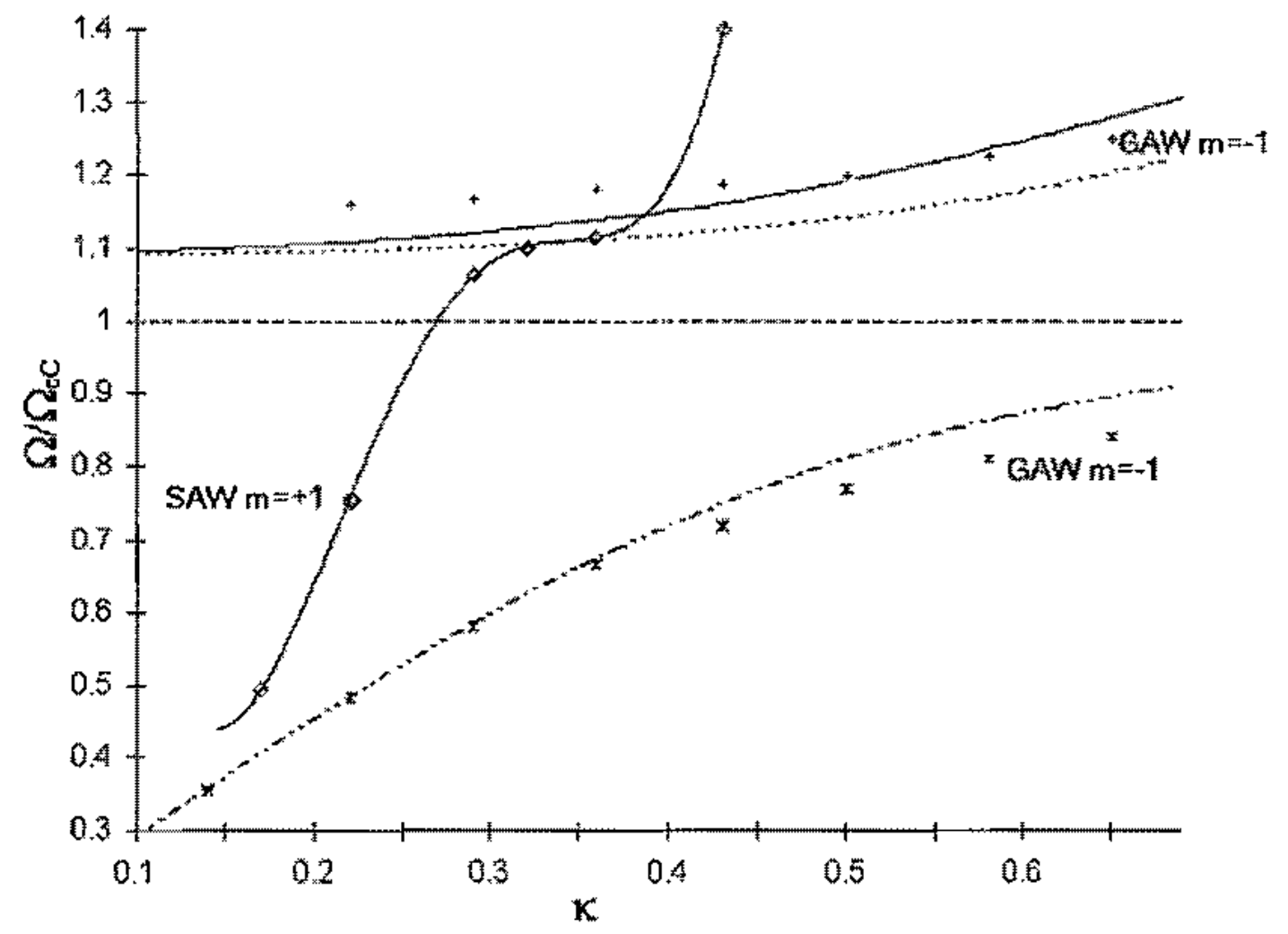

Fig. 2.8 Gráfico da frequêencia mormalizada do moxlo característico en un plasma de hidrogĕnio com $2 \%$ de impureza de carbono contra o número de onda toroidal negativo normalizado $\mathrm{k}$. Para $m=-1$, a frequầncia do limiar inferior do Contínuo de Alvén é representuda pela linha de ponto-traço-ponto, o

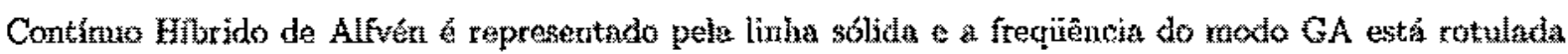
com cruzes; para $7 x=+1$, o Continuo Lubido de Alvén é representsdo através die linha pontilhada e a onda SA está rotulada com diamantes ligados por uma linba sólida. Os outros parâmetros de plasma säo 
iguais aos da Fig.2.4.

São identificados e comparados dois grupos de modos na Fig. 2.8. Fst䔀 graficadas a frequêtencia de limiar do contínuo de Alvèn para $m= \pm 1$, a freqüënéa da onda GA para $m=-1$ e a onda $S A$ para $m=+1$, que säo normalizadas à frequêencia ciclotrônica do carbono $\omega_{c C}$, contra o número de onda toroidal negativo normalizado, $\kappa=-\mathrm{cn} /\left(R_{0} \omega_{p H}\right)$. Ê mostrado que a onda $\mathrm{GA}(m=-1)$, que normalmente está situada abaixo do continuo de Alfvén, entra no contínuo quando a densidade dos lons de hidrogênio aumenta (ou o parämetro $*$ diminui).

A Fig. 2.8 mostra anda que o modo de Superficie de Alfvén $(m=+1)$ de fato cruza o contínuo de Alfvén, a lacuna do contínuo e Contímo Híbrido de Alfvén. Se a frequiência deste modo estiver no continuo, a estrutura do campo de RF é a de um típico modo de superfície com ponto de conversäo. Uma característica importante do modo de Alfún com $m$ positivo, é que seu caráter muda de um modo de superfície, quando estáa longe do contímu, para um modo global quando chega à regizo do contínuo. Próximo ao limiar do Contínuo Híbrido de Alfvém, a curva da frequêencia caracteristica deste modo está estendida junto à linha do contínuo e, aqui, a estrutura do modo da superficie é mudada dramaticamente para uma combinaçäo da onda GA e da onda SA, com ponto de conversäo, dentro do Contínuo Hibrido de Alfén. Neste caso, o centro do plasma pode ser aquecido também efetivamente como no caso da onda Combinada de Alfuern para $m=-1$ (veja comentúrios na Fig. 2.5 e Fig. $2.6 \mathrm{a}$ ).

Para modelar as experiencias de aquecimento por RF e a geracäo de corrente no tokamak TCABR, que pode simular as condiçōes de um reator de fusâo com plasma de Lois de componentes, é escolhida a mistura de plasma de Hidrogenio- ${ }^{3} \mathrm{He}^{+2}$ na proporção $66 \%-34 \%$, respectivamente. Esta proporção simula o Contínuo de Alfvén do plasma com mistura de D-T $50 \%-50 \%$ num reator (substituindo os valores relativos às quantidades 


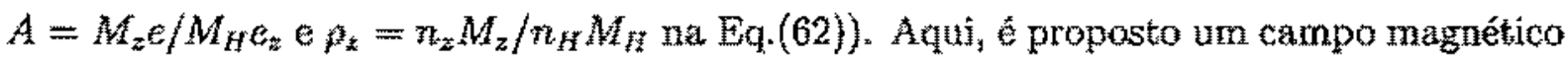
toroidal de $15 k G$ e $q_{\alpha}=3.3$. Na Fig. 2.9, é mostrada a impedăncia da antena contra a freqửência para os números de onda poloital $m=1$, e toroidal, $n=-4$. A impedância da antena é calculada nas redondeáa do limiar do Contimo Hibrido de Alfvén para um plasma com densidade $n_{\xi H}=1,9$ e $2 \times 10^{13} \mathrm{~cm}^{-3}$. São encontradas as condiçốs ótimas para o aquecimento dos elétrons e a geração de corrente no centro de plasma quando a onda SA entra no Contínuo Hibrido de Alfvén e se toma un MCA para a frequência de $19,01 \mathrm{MHz}$, com o ponto de conversảo de modos em $\tau \approx 0,1 a$. Neste caso, a distribuição da potência dissipada da onda na ressonância do MCA tambérn a mostrada na Fig. 2.9 .

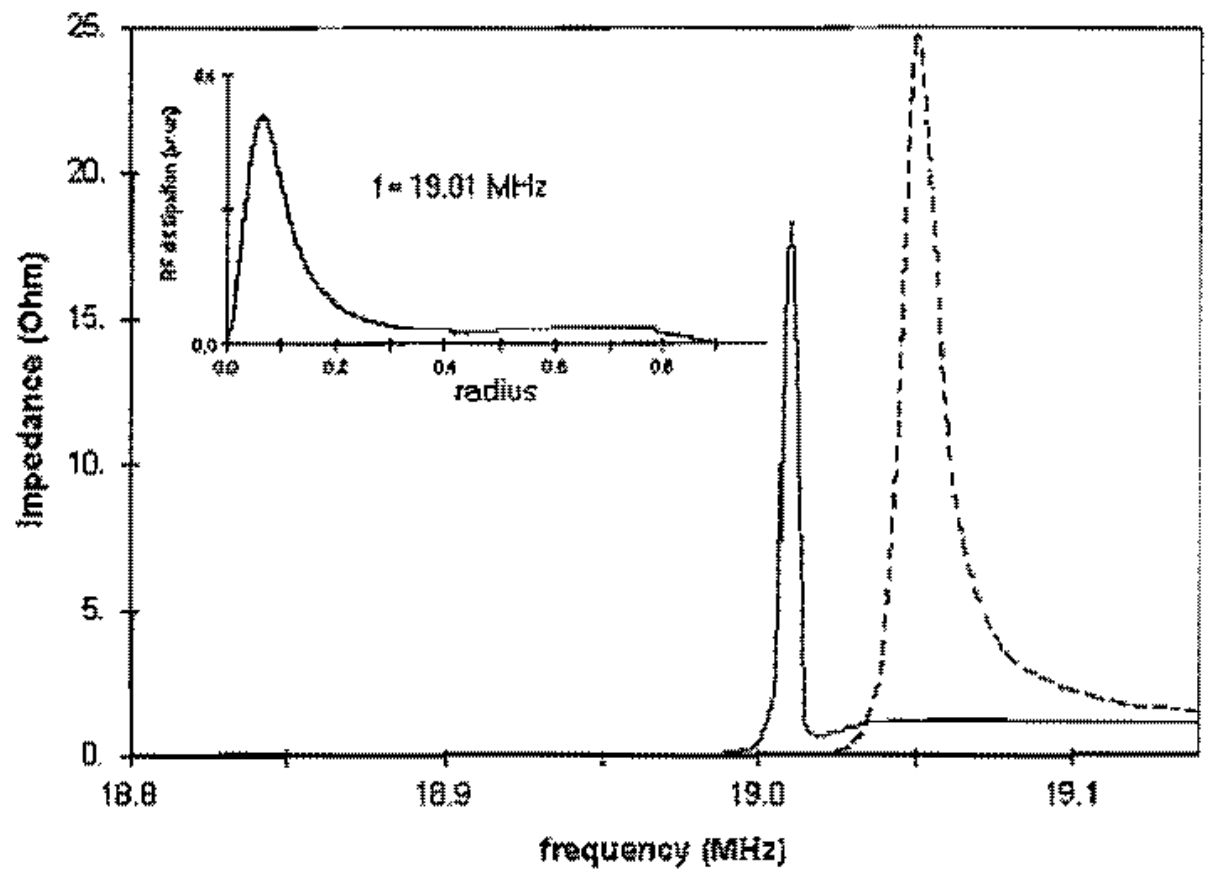

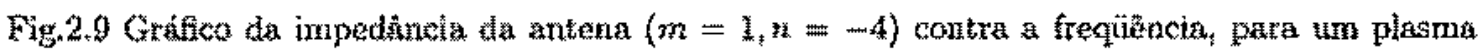

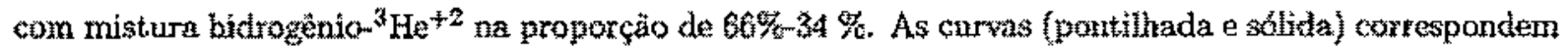

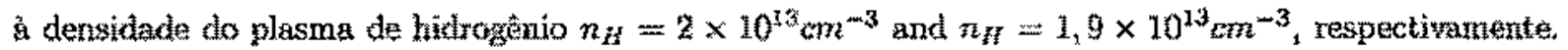

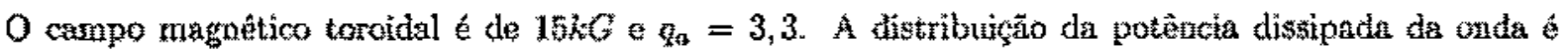

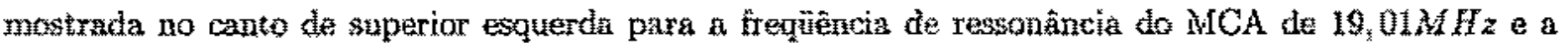
densidade de hiokrogênio de $1,9 \times 10^{13} \mathrm{~cm}^{-3}$. 


\subsection{Discussã̃o e Conclusão}

Usando as Equaçôes de Maxwell em uma geometria cilindrica para resolver o problema de valor de contorno para a excitaçäo do campo de RF, a ressonância da onda de Alfvén em um plasma magnetizado inomogêneo foi estudada analiticamente e numericamente. Com base nester cálculos, concluimos que

- Quando a freqüència do campo eletromagnético está no contínuo de Alfvén, dois tipos de ressonâncias de ondas de Alfvển sä́o excitadas. Uma delas está relacionada com o efeito de conversão de modos do campo de RF em KAW/QEAW (ou AWR local) e a outra ressonâneia está conectada com a excitaçăo direta de $\mathrm{KAW} / \mathrm{QEAW}$ na periferia do plasma. Estas duas ressonâncias estâa misturadas se a dissipaçăo da onda cinética de Alfvèn (ou onda quase-electrostática de Alfyén) estiver ausente.

- Levando em conta a dissipaşäo da onda pelo amortecimento eletrônico de Landau e/ou colisional, as ressonâncias locais da onda de Alfvén começam a aparecer quando o intervalo em $r$ onde há a dissipaçäo KAW/QEAW se torna menor que a distância entre o ponto de conversão e o centro do plasma (ou da periferia do plasma). Neste caso, o limite MHD da soluçāo geral pode ser encontrado e o valor total da potência dissipada calculada pelo modelo geral coincide com o valor calculado pelo modelo MHD.

- Outro ponto muito importante para entender a validade de um código MHD bidimensional aparece quando, por exemplo, devido a toroidicidade, sâo introduzidas múltíplas zonas de ressonância de Alfvén locais no plasmal ${ }^{11}$ ! Entäo, a faixa de dissipação da KAW/QEAW deveria ser menor que a distância entre duas ressonâncias de Alfvén vizinhas ${ }^{30 \mid}$, caso contrário, as ressonâncias das ondas de Alfvén podem ser sobrepostas e o valor total da potência dissipada calculada pelo modelo MHD pode diferir do valor correto.

Os cálculos e a análise apresentados neste capítulo foram baseados no modelo uni- 
dimensional da excitaçăo da onda de Alfvén e do aquecimento no contimuo. Geralmente, este modelo ê uma aproximação razóvel para as análises da onda Global de Alfvẻn e fenomenos de conversäo de modos em um plasma de tokamak com múltiplas espécies de ions cuando a freqüencia de gerador, w, esta situada no limiar do Contínuo de Alfvén. Neste caso, o parâmetro de toroidicidade é multo pequeno, $r / R_{n} \ll 1$, e efatos toroidais nảo säo importantes. Para evitar a ressonância ciclotronica efetiva dentro do Contínuo Hibrido de Alfvên, é preciso escolher a faixa de freqüência,

$$
\omega_{c Z, h f s}<\omega<\omega_{c h t, l f s}
$$

onde $\omega_{c Z, h f s}$ representa a frequêtencia ciclotrônica da impureza no lado de maior valor do campo magnético da coluna de plasma do tokamak, $\omega_{c z}(0) /\left(1-a / R_{0}\right)$, e $w_{\text {at }}$ ts representa $\omega_{\text {z }}(0) /\left(1+\right.$ a $\left./ R_{0}\right)$, a frequência ciclotronica de hidrogento no lado de menor valor do campo da coluna de plasma do tokamak. Esta limitaça da faixa de frequiencias às ondas de Alfvén de Supertucie com $m<0$ (estes modos sāo suprimidos nas zonas de ressonância ciclotrônica iônica) é imposta pela inomogeneidade espacial do campo magnético do tokamak. Näo há nenhuma limitação de faixa de frequêneias para ondas de superfficie com m positivo, isto é, as ondas rápidas, porque a dissipação destas ondas é rnuito fraca na resgononância cỉclotrônica iônica.

A limitação da faixa de frequênencia para modos caracteristicos GA,

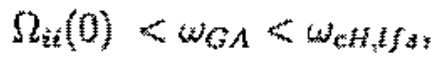

6 mais fraca porque estes modos só existem no centro da coluna de plasma. 0 limite superior desta desigualdade é trual à Eq. (64) porque a zona de frequêtencia de ressonäncia ciclotrônica do hidrogenio no lado de campo baixo näo permite a passagem de campos de RF de ondas GA $(m<0)$ da antena ao centro do plasma devido à polarização. $O$ limite inferior do modo QA é, te acordo com a Eq.(62), a frequência limite hibrida fon-ion no 
centro de plasma.

Os cálculos de dissipaçấo e dispersão da onda de Alfútun na parte superior do contínuo de Alvén no plasma de duas espécies de ions demonstraram uma influêncúa forte de impurezas no valor da impedância da antena, na posição da potência dỉssipada $e$ na dispersāo de modos característicos de Alfvén.

As frequênolas dos modos caracteristicos $G A$, perto do ramo mais baixo do contínuo de Alfyến en plasmas de vărias espêcies, saturam para grandes números de onda paralelo. Próximo ao ramo superior do contínuo de Alfvén, as frequêencias dos modos de superfície dependem fracamente do número de onda. A condiçäo de fraca dependência com o número de onda paralelo tern uma implicação importante e insinua um eficiente aquecimento do plastna com uma antenu que tem um largo espectro de comprimento de onda toroidal.

Säo encontradas condicões pará o aquecimento da coluna de plasma com ondas de Alfuén em plasmas de duas espécies para um tokamak de tamanho médio, cono o TCABR, quando os modos da onda GA caem dentro do Contínuo de Alfvén. Se qualquer uma destas condiçōes estiver satủsfeita, a major parte da potência dissipada de RF é enviada ao centro to plasma por conversão de modo na onda de Alvén cinética. Especificamente, com um nümero de modo toroidal $n=-4$ e um número de modo poloidal $n=-1$, om gerador com frequêtencia 0,29 vezes a freqüência ciclotronica do hidrogêtuio para o contínuo inferior de Alfvén (a densidade do plasma é de $3 \times 10^{13} \mathrm{~cm}^{-3}$ ) e 0,57 vezes a frequềncia ciclotrônica do hidrogênio para o Contínuo Hóbrido de Alfvén la densidate do plasma é de $\left.1,2 \times 10^{13} \mathrm{~cm}^{-3}\right), 0$ aquecimento central do plasma aconteceréc em um plasma de hidrogenenio com uma concentraçäo de impureza de carbono de 0,02 . Estas condiçóes podem ser satisfeitas para os outros modos toroidais, de $n=-4$ para -6 , de acordo com o diagrama de dispersão da Fig. 2.8 (com $\kappa=0,28$ e 0,355, respectivamente).

Com números de nodo poloidal positivo, também sấo encontradas as condiçōes para 
aquecimento central de plasmas de duas espécies com o Modo Combinado de Alfvén, quando estes modos caem dentro do Contínuo de Alfvén, perto do limiar. Especificamente, com um número de modo toroidal $n=-4$ e um número de modo poloidal $m=+1$, e uma freqüência do gerador de 0,83 vezes a frequiência ciclotrônica do hidrogênio para o Contínuo Hibrido de Alfvén (a densidade do plasma é de $1,9 \times 10^{13} \mathrm{~cm}^{-8}$ ). O acuecimento central do plasma acontecerá em um plasma de hidrogênio com a adição de uma concentração de ${ }^{3} \mathrm{He}^{+2}$ de 0,34 . Neste caso, a velocidade de fase, $2 \pi f / k_{\|}$, é aproximadamente 2,5 vezes a velocidade térmica dos elétruns. Nesta condição, a geraçăo de corrente por ondas de Alfvén pode ser alcançada muito efetivamente no contínuo híbrido inferior (veja comentário na Ref. [31]). Estas experiências de geração de corrente no tokarnak TCABR. podem simular a geraçāo de corrente com D.T em plasmas de reatores de fusăo. 


\section{CÓDIGO TOROIDAL}

\subsection{Histórico}

O Código ALFVEN fó escrito ern sua versão cilíndrica inicial, por G. L. CHEN e D. W. Ross em $1981^{\text {t2M }}$ e sofreu várias correçoes até a inclusăo dos termos de impurezas por W.Q. Li, em 198728). Em 1991 o cỏigo foi finalmente adaptado (por W.Q. Li) para o acoplamento de trës modos poloidais a sua forma bidimensional foi definida com modificações introduaidas por D.W. Ross, W.H. Miner, J. C. Willey e R.R. Mett até setembro de1992. Fó trataida para o instituto de Física da USP em novembro de 1997 por G. S. Amarante Segundo, apơs um estágio na Universitude do Texas em Austin, onde o código foi desenvolvido. Este ultimo introduzilu algumas modificaçös no código, principalmente adicionando o módulo de cálculo de forças ponderomotoras.

\subsection{Introduçâo}

Para grande parte das situaçoes fisicas que se quer modelar num lokamak, uma primeira aproximação local ou um modelo em geometria cilíndrica oferece uma compreensão qualitativa dos fenômenos bastante satisfatónia. Na busca de refinar os resultados já obtidos ou, principalmente, quando a tarefa proposta é a determinação quantitativa de regines otimizados a serem werificados experimentalmente, é necessărio ir mais adiante na inclusäo de efeitos resultantes de um modelamento mais realista do sistema.

Neste capítulo, e nos seguintes, seräo apresentados vários resultados que comprovam haver diferengas muito importantes das situaçỏes de interesse calculadas com um código de aproximação cilíndrica e com o codigo toroidal aqui apresentado. O acoplamento de modos poloidais permite uma avaliação aproximada de um sistema toroidal real, mas introduz uma série de mudanģa nos resultados, particularmente nos perfís dos campos 
quando dos modos Globais de Alfvén.

Neste capítulo há uma suscintá descriçầo do funcionamento do código (com os cálculos apresentados no Apëndice E) e säo listados e discutidos os principais resultados.

Evidentemente, mudanças podem ser efetuadas no código para que ele se aperfeityoe ainda mais, e algumas sugestöes neste sentido sâ feitas no Capiculo 5 .

\subsection{O Código}

O código cinético toroidal usado neste trabalho b baseado no modelo toroidal de plasma simplificado já presente nas Rels.[32]. O código calcula os campos das ondas de Alfvén e a dissipaçäo em plasmas magnetizados com duas espécies de fons e apresentanto superficies magnéticas circulares e concêntricas. Säo usadas coordenadas pseudo-toroidaís (definidas via as transformacoses $R=R_{0}+r \cos \theta, \zeta_{1}$ \& $Z=r \sin \theta$ ), onde $R_{0}$ é o raio malor da seção transwersal da coluna de plasma de rajo menor $a$ e $Z$ é a coordenada animutal do sistema toroidal, conforme mostrato na Fig. 3.1 .

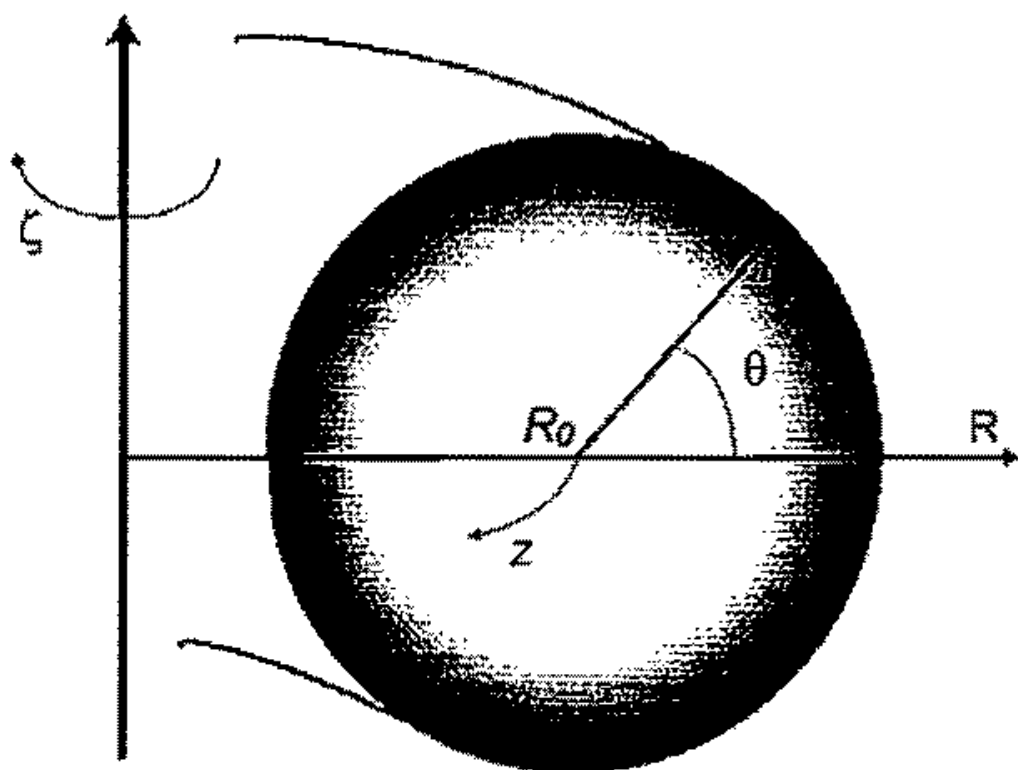

Figura 3 - Esquema das coondenadns toroidais do código bidimensional. 
As equaçōes de Maxwell acopladas (repetindo a Eq.(9)),

$$
\frac{w^{2}}{c^{2}} \vec{E} \cdot \vec{E}-\vec{\nabla} \times \vec{\nabla} \times \vec{E}=0
$$

são resolvidas no sistema de ccoordenadas acima descrito, onde, $\overleftrightarrow{\epsilon}$ é o tensor dielétrico. A análise é simplificada usando a aproximaçäo de pequena toroidicidade, $\epsilon=r / R_{0} \ll 1$. Neste caso, as componentes do campo magnético de equilibrio podem ser representadas na forma

$$
\vec{B} \approx B_{0}(\widetilde{\zeta}+\vec{\theta} \delta)(1-\epsilon \cos \theta)
$$

onde $\delta=\epsilon / q, q=r B_{0} /\left(R_{0} B_{\theta}\right)$ e $\hat{\zeta_{1}} \hat{\theta}$, são os vetores unitários nas direçōes toroidal e poloidal, respectivamente. Os efeitos das colisões, do raio iönico de Larmor finito $\left(\rho_{L}=\right.$ $\left.v_{T i} / \omega_{\mathrm{c}}\right), \omega / \omega_{c i}$ (parâmetro de Hall, onde $\omega_{c i}$ é a frequâencia ciclotrônica iônica) finito, do amortecimento de Landau eletrônico, e de bombeamento magnético por tempo de trẳnsito (trunsit time magnetīc pumping) säo levados em consideraçāo na dispersão e dissipaçāo da onda. Todavia, é feita a hipótese de que os raios de Larmor eletrớnico e iônico são ambos muito menores que o comprimento radial característico das quantidades de equilíbrio do plasma e dos campos de RF. Apesar de o código toroidal, na aproximação utilizada, acoplar apenas os modos principais e seus vizinhos mais imediatos, $m$ e $m \pm 1$, um múmero arbitrário de modos (nẫo acoplados) pode ser usado para revelar efeitos cinéticos na excitaçāo dos campos de RF. Com a aproximaçầ de pequena toroidjeidade, o tensor dielétrico, $\overleftrightarrow{\epsilon}$, é reduzido à sua forma local cilíndrica com correçốes toroidais de primeira ordem (Ver Apêndice B). Os outros parâmetros, como cisalhamento magnético, perfil de densidade, e distribuçōes de temperatura sāo as mesmas do Capítulo 2.

O sistema dado pela Eq.(65) pode ser descrito por um operador matricial usando um sistema local com coordenadas paralela e perpendicular às linhas de campo magnético. 
Os vetores unithatios correspondentes säo

$$
\hat{e}_{/ /}=\frac{\hat{C}+\hat{b} \delta}{\left(1+\delta^{2}\right)^{1 / 2}} \text { and } \vec{e}_{1}=\hat{e}_{\|} \times \hat{e}_{r *}
$$

A álgebra é simplificada impondo a condição de corrente fechada,

$$
\vec{\nabla} \cdot \vec{J}=\frac{1}{r R} \frac{\partial}{\partial r} r R I_{r}+\frac{1}{r R} \frac{\partial}{\partial \theta} R J_{0}+\frac{1}{R} \frac{\partial}{\partial \zeta},{ }_{\zeta}=0
$$

onde $J_{\zeta}=\left(B_{\zeta} J_{1}-B_{0} J_{\perp}\right) / B$ e $J_{\theta}=\left(B_{\zeta} J_{\perp}+B_{0} J_{1}\right) / B$ para a corrente excitada pela onda. Da Lei de Aripere com a corrente de deslocamento desprezada, a corrente de plasma á dada nor

$$
\vec{J}=\frac{1}{i \mu_{0} \omega} \frac{w^{2}}{c^{2}} \stackrel{\leftrightarrow}{\chi} \cdot \vec{E}
$$

Usando a Eq. $(68), E_{11}$ pode ser expressa cm termos de $E_{r}$ e $E_{1}$, a a parte $E_{4}$ na Eq.(65) pode ser eliminada, reduzindo a ordem do sistema de equaçöes. Ele é resolvido como um problema de valot de contorno, para os componentes $E_{j}$ do campo de RF no plasna. Tal tarefa é levada a cabo construndo-se um equaçầo matricial para representar as equaçőes diferenciais de segundawordem acopladas na forma de Einstein,

$$
\frac{A_{\mathrm{i}}(r)}{r} \frac{\partial}{\partial r} r \frac{\partial}{\partial r} E_{j}+B_{i j}(r) \frac{\partial}{\partial r} E_{j}+C_{i j}(r) E_{j}=S_{i}(r)
$$

onde $A_{i \hat{i},} B_{\text {, }}, C_{i j}$ são os coeficientes da matriz que operam as componentes do carnpo elétrico e $S_{i}$ é um vetor de fonte onde sâo inclutdas as condiçẩo de contorno. A notação compata resultante do conjunto de equactes matriciais é determinada por

$$
\widehat{L} \vec{E}=\vec{L}_{0} \vec{E}+\left(2 \frac{r}{R_{0}} \cos \theta \hat{L}_{S}+2 \frac{r}{R_{0}} \sin \theta \hat{L}_{A}\right) \vec{E}=0
$$

que consiste em uma parte cilíndrica e duas partes toroidas proporcionais a $r / R_{0}$. Os operadores $\hat{L}_{0}, \hat{L}_{S}, \hat{L}_{A}$ säo explicitados na Ref. [20]. As equaços sofrem uma tranatormaçäo de Fourier e, como resultado, podem ser representadas na forma de uma equaçäo matricial 
$6 \times 6$ com acoplamento harmonico poloidal de primeira ordem,

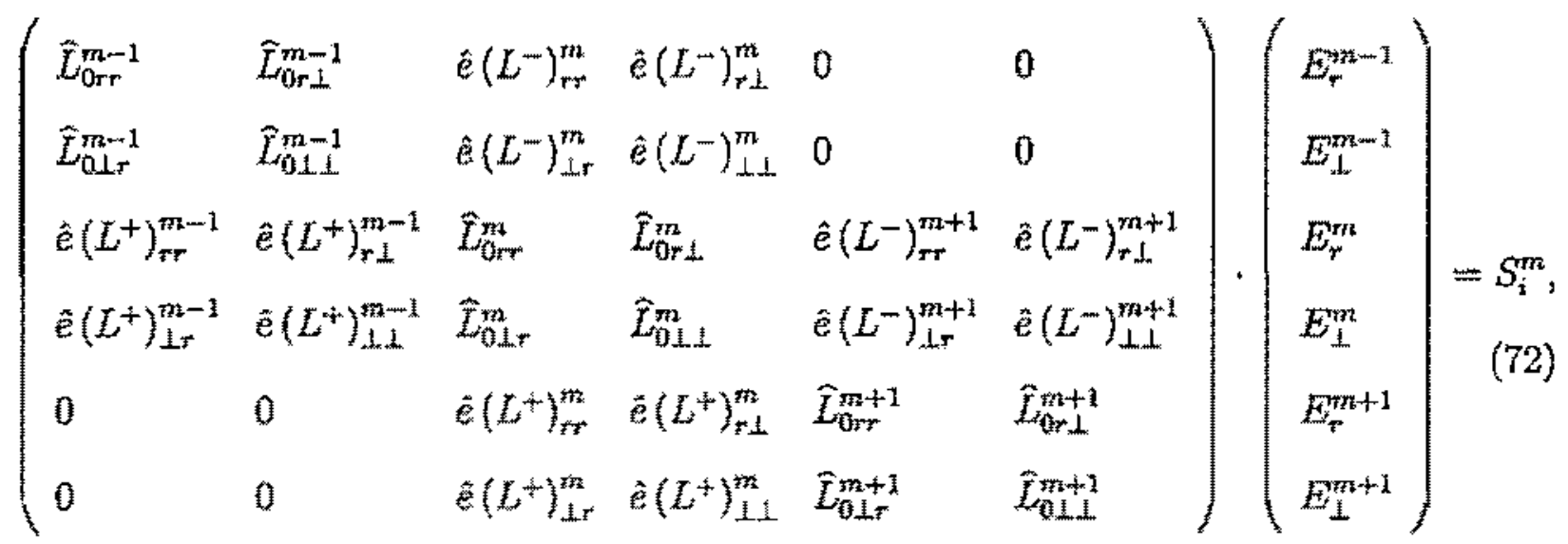

onde $L^{+}=\hat{L}_{S}+\hat{L}_{A} ; L=\hat{L}_{S}-\hat{L}_{A}$. As condiģöes de contorno são especificadas no vetor $S_{i}^{m}$ e o problema é resolvido finalmente por uma rotina spline-Galerkin cúbica 20 ! Nos cálculos mostrados aqui, säo usados 700 pontos radiais dispostos numa grade com pontos menos espaçados para $r$ próximo de a (a disposigäo dos pontos é configurável) e 3 harmônicos poloidais. O código gera os perfis das componentes dos campos elétrico magnético a o perfil de deposiçǟo de energia $\left(\vec{J} \cdot \vec{E}=\vec{E}^{*} \cdot \vec{\chi} \cdot \vec{E}\right)$. Levando a cabo uma integraçäo radial, a impedincia da antena pode ser diretamente calculada.

E suposto que os campos de RF são produzidos por umá antena de corrente superficial no limite cilindrico (com modos poloidal e toroidal de números $M$ e $N$, respectiva mente). As componentes $\zeta$ e $\theta$ da corrente superficial, situada em uma superficie de raio b. säo dadas por

$$
J_{\zeta, \theta}=J_{\zeta, \theta}^{(M, N)} \delta(r-b) \exp [i(M \theta+N \zeta-\omega t)]
$$

onde $J_{x, \theta}^{(M, N)}$ ê a amplitude constante do harmónico $M, N$ e $\omega$ é a frequência do gerador. A corrente na antena é especificada através de condiçós de contorno nas componentes do campo magnético de RF nas espiras de raio b. Em tomo da superfície da antena há um retizo de yácuo envolta por uma parede condutora de raio $d$. Os campos no vácuo säo calculados analiticamente e normalizam os campos de plasma através de condicöos de contorto apropriadas no raio $a$. 


\subsection{Dissipação da onda de Alfvén}

A câmara de vácuo do tokamak TCABR tem um corte transversal retangular a a antena é feita por um conjunto de semi-círculos poloidais em fase que produzem um campo de $2 F$ helicoidal $[33$. Porém, as simulaçōes podern ser levadas a cabo usando uma geometria de tokamak de cotte tranguersal circular, con os seguintes parânetros: raio da coluna $a=0,18 m$ raio maior $D_{a}=0,61 \mathrm{~m} ;$ raio da superficie da antena $b=0,184 \mathrm{~m}$; raio da parede $d=0,23 \mathrm{~m}$

Em sua fase inicial, a máquina será operada com um campo magnético toroidal

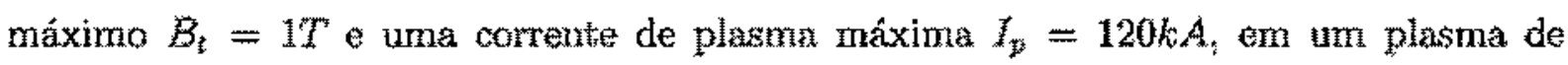
hidrogênio. Os cálculos sễo efetuados supondo-se um perfil de temperatura dado por

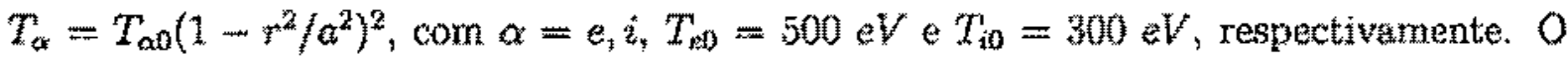
perfil de densidade eletrónica é da forma $n_{e}=n_{0}\left(1-r^{2} / a^{2}\right)+n_{a}$, com $n_{0}=3 \times 10^{19} m^{-3} \mathrm{e}$ $n_{a}=1 \times 10^{18_{m}}{ }^{-3}$. E excolhido um valor alto para a densidade de pedestal $n_{a}$ para evitar muitas ressonâncias de Alfvên perto da periferia do plasma (ctal expediente também utilizado no código cilíndriço, página 44). A densidade iônica é escolhida de forma a satisfazer a exigencia de neutralidade de carga, $Z_{n_{Z}}+n_{i}=n_{k_{z}}$ onde $n_{z}$ e $n_{i}$ designam as densidades da impureza e os jons de hidrogênio (ou deutério), respectivamente. O perfil da impureza pode ser especificado arbitrariamente, porém, nos cálculos mostrados neste trabalho, os perfís da impureza são proporcionais aos dos lons. Finalmente, supöt-se o perfll de densidade de corrente na forma $j=j_{0}\left(1-y^{2} / a^{2}\right)^{3}$ e os valores da transformada rotacional inversa na faixa $0,8 \leq q_{0} \leq 1,6$, no eixo magnético, $e 3,2 \leq q_{3} \leq 6,4$ na periferia. A real estrutura do novo sistema de antenas do TCABR é levada em conta nos coeficientes calculados na Ret.[33]. Un único modo de antena é considerado, que corresponde à componente principal dada pela análise de Fourier do sistema da antena Teal. 
É considerado inicialmente o caso do plasma de hidrogênio puro. Na Fig. 3.2 é apresentada a curva da impedância $Z_{M N}$, plotada como função da frequiência do gerador e normalizada à freqửencia do limiar do contínuo de Alfvén para cada modo,

$$
\omega_{t h}=k_{4} c_{A} / \sqrt{1+\left(k_{\|} \mid c_{A}\right)^{2} / \omega_{c}^{2}}
$$

para diferentes nủmeros de modo toroidal, $N$, do espectro de antena. $k_{i}=(n+m / q) / R_{0}$ é o número de onda paralelo, to e $n$ são os números de onda poloidal e toroidal, respectivamente, e $c_{A}=B_{0} / \sqrt{\mu_{0} m_{i} n_{i}}$ é a velocidade de Alfvén (Os números de onda toroidal e poloidal sầ representados através de letras maiúsculas quando citado o modo principal da antena e por minúsculas quando citados os modos de fato excitados no plasma). Os valores dos parămetros pertinentes são determinados nas legendas das figuras.

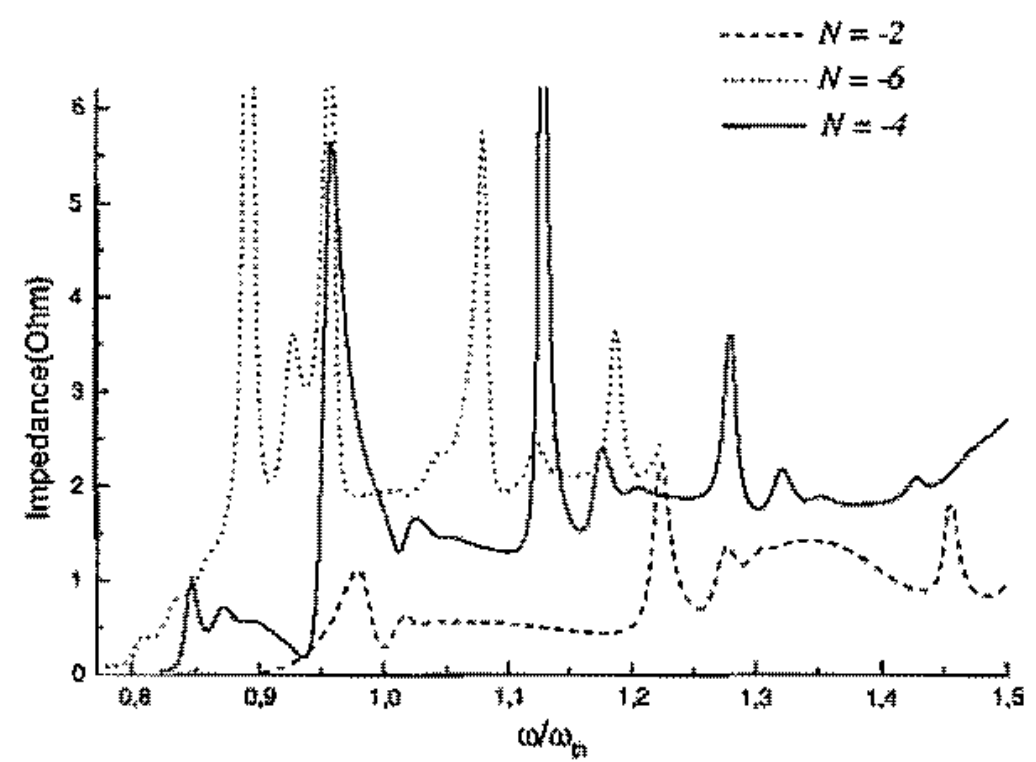

Fig. 3.2. Impedâneia da Antena para um plasma de liưrogênio contra a tregilência nomalizada para a frequiêencia mínima do contínuo de cada modo ( $\left.\omega_{\text {an }}\right)$. As curvas de impedância (pontilhada, sólida a espaçada) correspondem aos números de onda toroidal diferentes $N=-2,-4,-6$, respectivanente, do espectro da antena pata número de onda polotal fxo $M=-1$. Os parấmetros do plasma sắn $n_{0}=3 \times 10^{19} m^{-3}, 70=500 \mathrm{eV}, B_{t}=1.7,7_{0}=1.12 q_{0}=4.4$

Como esperado, a ressonância da onda Global de Alfvén cilíndrica $m=-1$ dividese ern três ressonâncias globais (con números dos modos cilindrícos equivalentes $n=$ 
$0,-1,-2)$, devido ao acoplamento dos modos poloidais. E importante notar que a impedância da antena aumenta com os números de onda toroidal $N$ e que o modo $N=-6$ tem una eficiência mais alta na deposiçäo de energia no plasma. Unn valor tẩo alto do número toroidal nảo foi investigado em Lausanne. O novo sistema de antenas do TCABR. permite a excitação deste modo para investigar o desempenho predito.

O efeito da absorçấo ressonante dos modos vizinhos, acoplados no perfil de potência absorvida, pode ser avaliado prontamente, considerando a distributça da potencia dissipada nos picos das ressonanous $G$ correspondentes a $m=0,-1$ e -2 , mostrados na Fig.3.2. Este efeito é demonstrado na Fig. 3.3 para o modo $N=-4$. Claramente, 0 amortecimento do contínuo na ressonância local para $m=0$ supera o amortecimento de Landat da Ressonância GA prircipal $m=-1$, causando um perfil largo indesejável de deposição de energia perto da periferia do plasma. A benéfica deposiçăo de potência central para $m=0$ é um efeito puramente toroidal que näo pode ser predito através de cálculos cilindricos. Para um valor alto do número de onda torodal, $N=-6$ por exemplo, o modo vizinho mass distante move-se em direçăo à ressonância principal e pode acontecer um sobreposiçäo das ressonancias globais e locainz, o que resultaria num perfil de deposição de potěncia mais largo do modo de ressonância principal. 


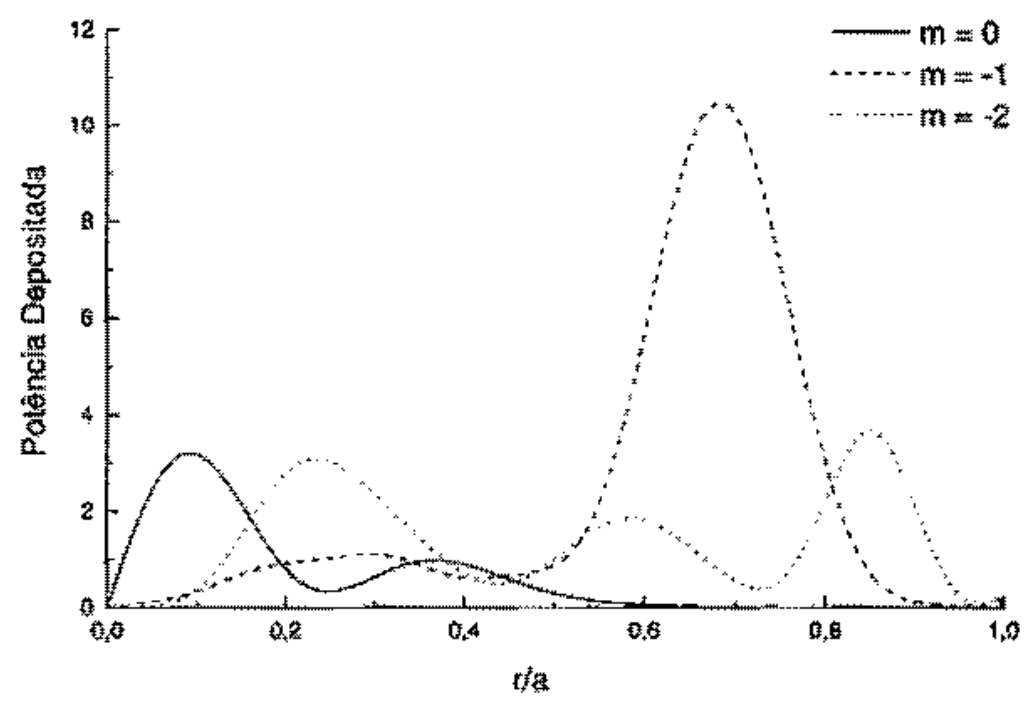

Fig. 33. Distribuiçăo de potência da onda dissipado sobre a coordenada radial (rormallazada como raï da coluna de plasma) para ressonâncias $G A$ para $N=-4, m=0,-1,-2$, que săo apresentadas na Fig, 3.2 (com as frequêneias $4.07,4.68$ e $5.65 \mathrm{MHz}$, respectivamente).

A competição entre o contínuo dos modos vizinhos e o amortecimento direto da onda global pode ser enfatizada considerando uma temperatura eletrônica muito baixa. A distribuição da dissipação de potễncia para temperatura eletrônica central $T_{e}=100 \mathrm{eV}$ e $T_{\mathfrak{i}}=60 \mathrm{eV}$ é mostrada ná Fig.3.4. Todos os outros parâmetros são iguais aos da Fig. 3.3 (lembrando que $T_{e}=500 \mathrm{eV}$ o $T_{i}=300 \mathrm{eV} \mathrm{Ha} \mathrm{Fig.} \mathrm{3.3)}$

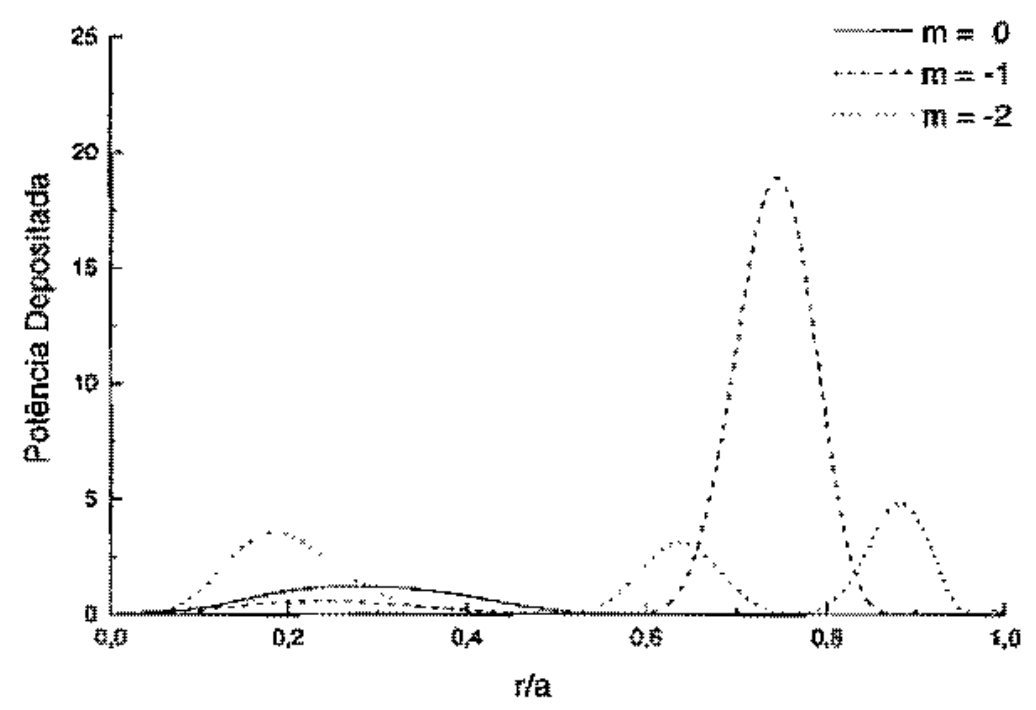

Fig. 3.4 Distributcão da potuncia dissipada pala onda sobre a coordenada radial (normalizada),

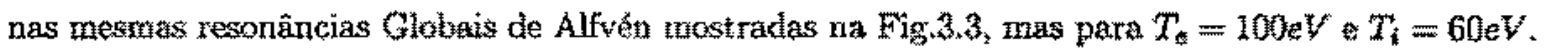


Para baixas temperaturas, a estrutura de deposiçẫo de energia de todos os modos é fortemente afetada, diminuindo-se os efeitos cinéticos na ressonância principal e aumentando o valor do pico de amortecimento da recsonância no contínuo do modo vizinho em comparação com o caso $T_{e}=500 \mathrm{~V}$. Para $m \neq 0$, a onda Cinética de Alfvén, que se propaga a partir de uma superfície ressonante, tem uma largura de dissipaçăo

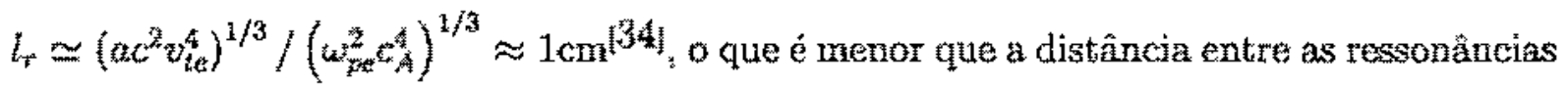
de Alfẻn locais e o centro do plasma. Entäo, a estrutura osclatória radial dos modos cinéticos năo aparece. Como previamente mencionado, os efeitos toroidás rão modificam substancialmente a posiçằ dos picos de ressonancia na curva de impedancia da antem na predita por modelo cilíndrico. Isto pode ser visto variando-se o valor de gu, com os outros parânetros mantidos fxxos. Pode-se ver a impedancia da antena como funçäo da freqü̉ncia do gerador para diferentes valores de $q 0$ na Fig. 3.5. Neste caso a frequência năo é normalizada porque a freqüência do limiar do contínuo varia com o valor de qo. Como esperado, a frequiência de ressonância para o modo poloidal $m=0$ permanece quase inalterado. Para cada modo poloidal $m \neq 0$, a freguêencia da ressonăncia GA é mum dada de acordo com a dependência da freqüência do limiar do número de onda paralelo, $k_{1}=(n+m / q(r)) / R . O$ valor da impedâneia diminut com o aumento de $g_{0}$. 


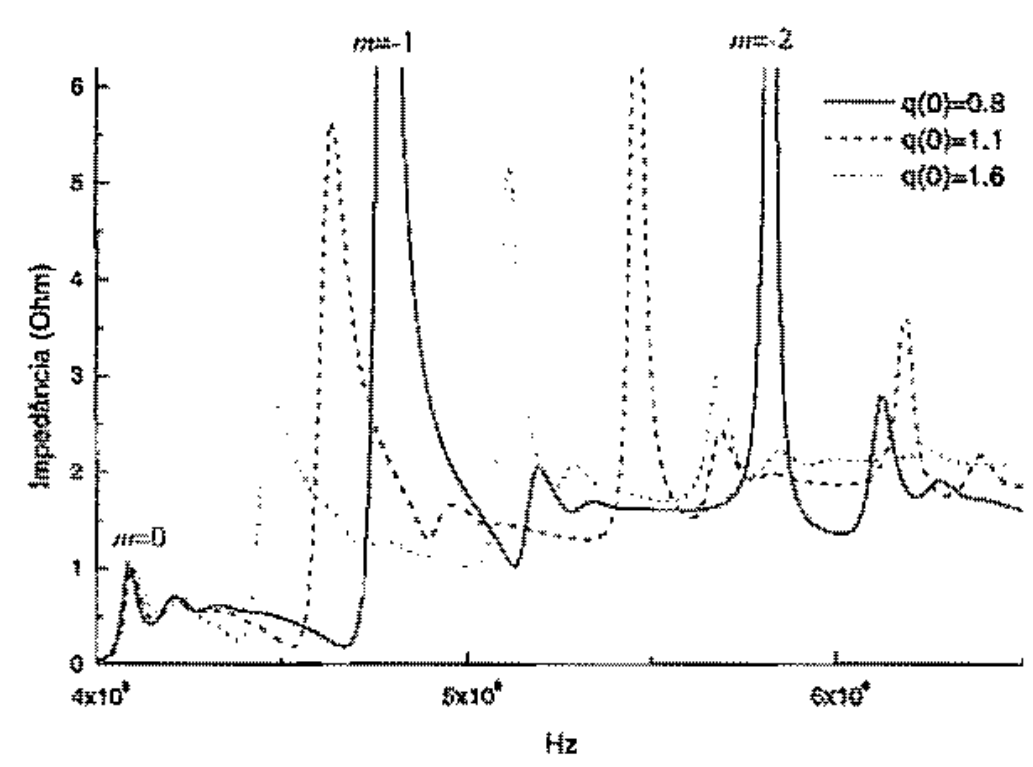

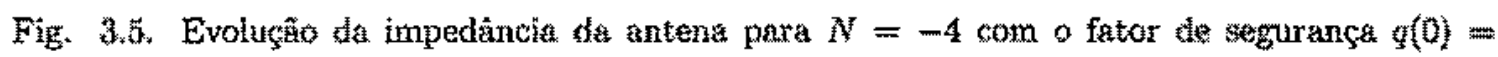

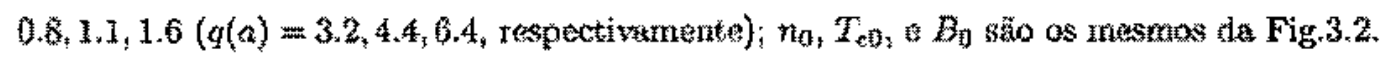

Nota-se que a curvas de impedancia nas Figs. 3.2 e.5 são calculadas usando-se três harmōnicos, mas estas curvas näo dapendem do número de modos vizinhos (este efeito fơ verificado com até \pm 10 modos vizinhos) no intervalo de frequî̀̂̀ncias que começa da frequêência do pico de ressonância $G$ A do modo $m=0$ até o modo $m=-3$.

\subsection{Efeito de impurezas na dissipaçäo da onda de Alfvén}

Üsando o código uridimensional, jâ foi mostrado que pequenas porçöes de impurezas, tais como deuterio e carbono em um plasma de hidrogênio, modificam a dispersão tanto da onda Global de Alvén como do contiono de Alvén abaixo da frequênencia ciclotrônica do hidrogênio 35 . Neste caso, a frequîencia de ressonância GA pode depender fortemente do número de onda toroidal e a onda Clobal de Alfvén pode entrar no contínuo. Aqui, este resultado é contirmado com o modelo cinético torotial. Na Fig. 3.6, são apresentados os resultados dos cálculos da impedància da antena $Z_{M N}$, com ambos os códigos, para um plasma de hidrogênio e para plasma com uma população de $2 \%$ de impuraza de carbono. 
Considerando a forma aproximada do campo magnético toroidal (Eq.(66)), a dependência espacial da ressonância cíclotrônica da impureza aparece como um termo de primeira ordem, $\sim\left(r / R_{0}\right) \cos \theta$. Consequientemente, a superfície da resonância ciclotrônica da impureza aparece no código como uma superfície circular com centro deslocado. Esta superfície é considerada distante da superficie de conversäo de modos $\mathrm{e}_{\text {, neste caso, a }}$ dissipaçäo ciclotrônica ế pequena e pode ser desprezada.

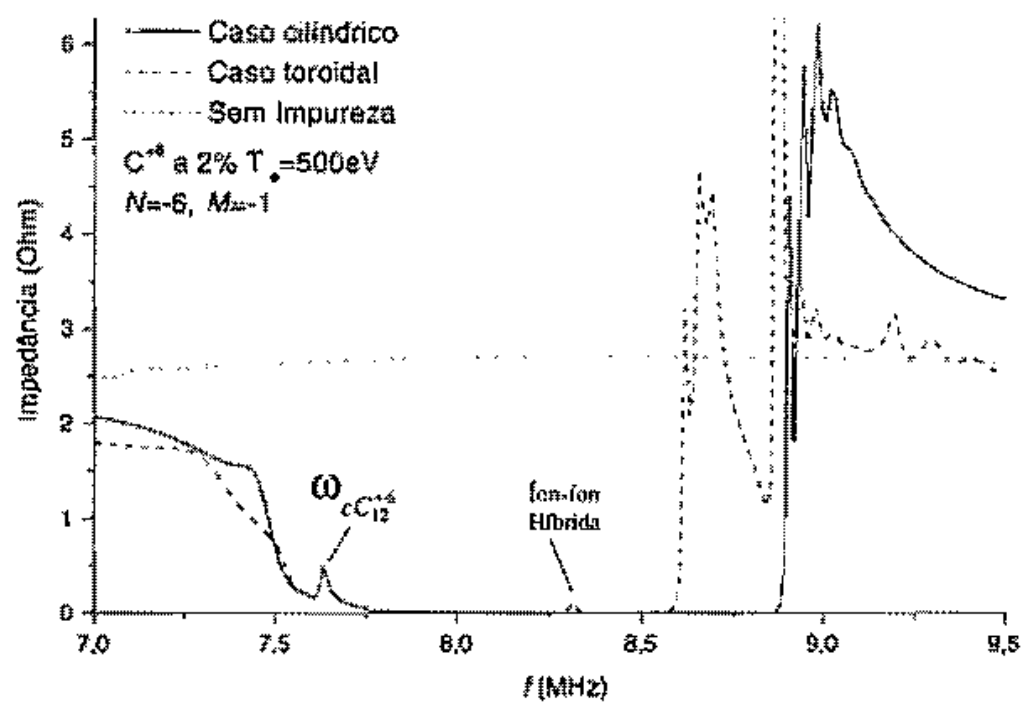

Fig.3.6. Impedäncia da autena para $N=-6, M=-1$ contra a frequêencia em $M H z$ para um plasma de hidrogênio com impureza zarbono. As linhas de impedânncia sólida to pontillhada correspondem a concentraçes de carbono relativas $n_{C} / n_{Q}=0,0$ e 0,02 , respectivamente, no modelo unidimensional, $e$ a linha tracejada representa os cátculos bidimensionais para $n_{C} / n_{0}=0.02$

Verifica-se que uma pequena populaģấo de impurezas como deutério ou carbono em um plasma de hidrogênio modifica o contínuo de Altuén substanciajmente. Por causa de impurezas, uma lacuna aparece nas curvas de impedância sobre as ressonâncias ciclotrônica da impureza, $\omega \approx \omega_{c z}=7,65 M H z$, tanto nos cálculos unidimensionais como bidimensionais. Porém, no modelo toroidal, uma dissipaçäo adicional aparece sob o Contímo Hibrido de Alfvén, com $f=8,88 \mathrm{MHz}$, para os números do modo cilíndrico $n=-6, m=-1$, por causa do efeito do acoplamento de modos poloidais. Esta dissipaçäo é devida ao Contínuo Hibrido de Alfvén con números de modo $n=-6$ e $m=0^{36}$. 
A presença do contínuo hibrido é bastante benéfica para o perfil de potência dissipada. Isto pode ser visto calculando-se o perfil de deposição para a ressonância GA principal, com $n=-6$ e $m=-1$ na frequência $8,87 \mathrm{MHz}$, mostrada na Fig. 3.6. A distribuiçāo correspondente da potência de onda dissipada é vista na Fig. 3.7. Vê-se que devido ao fato de a GAW entrar no Contínuo Híbrido, a deposiçăo da potência perto do eixo magnético è aumentada substancialmente, enquanto a deposiçāo dos modos vizinhos na borda do plasma é diminuída.

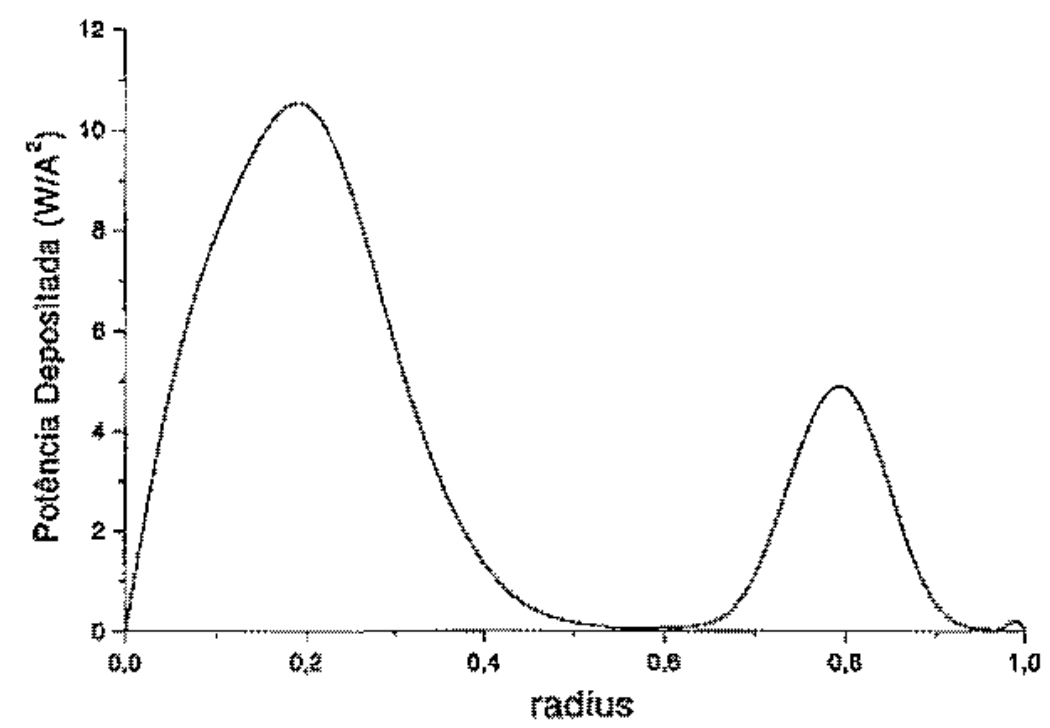

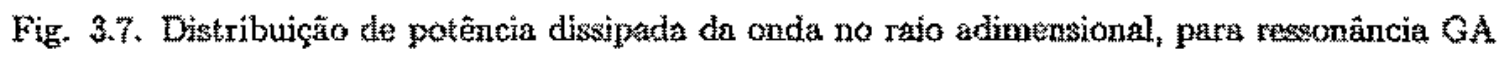
$m=-1$ e $n=-6$, wa freqüência de $8,87 M H z$ apresentada na Fig. 3.6 para un plasma de hỉdrogênio com impureza carbono wa concentraçần relativa $n_{c} / n_{0}=0.02$. 


\section{FORÇAS PONDEROMOTORAS}

\subsection{Utilidade e Importância}

A geração näo indutiva de correntes em tokamaks é um mecanismo essencial para a viabilizaşāo de reatores termontucleares e a investigaçãa nesta área tem uma longa história (veja revisöes [37, 31]). As experiências mostraram que a geraçăo de corrente (e de fluxo no plasma) pode ser resultado da dissipaça de diferentes tipos de ondas (ciclotronica eletronica, ondas hibridass inferiores, rápidas e de Alfún\}. Recentemente, melhorias no confinamento de energia foram alcançadas com barteivas de transporte internas (ITB - Internal Transport Barriers), criadas atraves de injeção de partículas neutras (NB) e por acuecimento por ondas na faixa da frequência ciclotrônica ionica (ICRF) em todos os grandes tokarxaks, como Dublet-III.D, Joint European Torus (JET). TWTR e outros (veja, por exemplo, [38] e o review [6]). Estas barreiras de tansporte aparecen na metade do raio menor do plasma com um cisalhamento (shear) negativo do carnpo magnetico (perfil de corrente oco) e uma rotaçâo toroidal e poloidal cisalhada da coluna de plasma para reduzir a turbulencia do plasma (veja a discussiono na Ref. [39]). Neste caso, as caracteristicas de dispersão de ondas de baixas frequêencias (como âtrift-Alfyén zanes) também podem ser modificadas (inclusive con a supressão de instabildades) por causa do termo de convecção $\mathrm{U} \cdot \nabla \bar{u}_{1}$ onde $\mathrm{U}$ é a velocidade de fuxo do plasma e $\tilde{u}$ a velocidade oscilatória.

O modelo físico mats simples de geraģäo de corrente e fluxos no plasma é o da transformacäo do momento dissipado da onda, $P / y_{\mathrm{p}}$ em momento dos elétrons do plasma. Este momento c transferido devido a collsōes elétron-ion, $m_{a} p_{e} n_{e} U_{c}$ (da energia da onda $E=h t w$, e do momento linear $\vec{p}=\hat{h} \vec{k}$, a potencia dissipada para $N$ fótons é expres sa pela força de transferência de momento $\vec{F}=W \vec{k} / \omega)$. Finalmente, em condiçốcs estacionárias, o equilbrio do momento conduz ao valor da densidade de corrente e fuxo 
gerados, jod e $U$, que sẫo governados palas equaçöes:

$$
j_{c d}=e W^{(\mathrm{c})} /\left(m_{e} \nu_{e i} \nu_{p h}\right), \quad \vec{U}=r_{E} W \vec{k} /\left(w_{m} m_{i} n_{i}\right)
$$

onde $\tau_{E}$ é a vida média do momento das particulas, a velocidade de ase $v_{p h}=\omega / k_{1}$ tem valor próximo ao da velotidade de Alfvén, $c_{A}=B_{0} / \sqrt{4 \pi<n_{i} m_{i}}>, \nu_{e i}$ a frequêencia de colisăo elétron-ion, $W^{(e)}$ è a densidade de potência dissipada nos elétrons, $e$ e a carga dos elétrons e $n_{\alpha}$ e $m_{\alpha}$ säo a densidade e a massa de elétrons ou íons, respectivamente. Esta equação simples é valida para quando a velocidade de fase é menor que a velocidade térmica dos elétrons, $w_{T e}=\sqrt{T_{a} / m_{e}}$. O resultado, válido para una gama inteira de velocidades de fase, é apresentado na Ref. [40]. Alta efictência na geraç̧o de corrente foi demonstrada tanto para ondas de Alfuén com velocldade de fase muito pequena, etph tre, como para velocidade de fase muito grande, $v_{p h}$ s w the

É bem sabido que há dificuldades significativats para o uso da geraçăbo de corrente por ondas de Alfuén em um reator-tokamak. No caso de baixas valocidades de fase, a eficiência da geraçăo de corrente cai no regime de bunana como resultado da absorçăo do momento da onda pelas partículas aprisionadas por causa da forte fricçáo entre elétrons livres, que formam a corrente, com os elétrons aprisionados e ions 337,41$]$. Por outro lado ${ }^{421}$, no regime banana, o momento dia onda que e transmitido para os eletrons aprisionados deveria ser conservado como momento toroidal canônjoo dos elétrons,

$$
\frac{R}{R_{0}} m m_{\|}+\frac{e}{m_{e}} \int_{0}^{r} B_{0}\left(r^{r}\right) d r^{\prime}
$$

Por causa da força de transferência de momento paralela, os elétrons podern sofrer

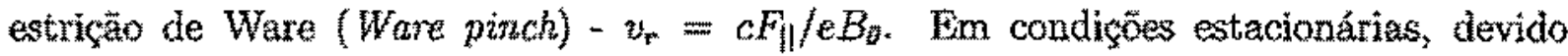
à difusäo colisional (estrição inversa de Ware - inverse Ware pinch- por colisöes), este momento pode parcialmente (aproximadamente $50 \%$ do momento da onda) contribuir para a corrente de elétrons livres. 
As esperanças de uma geraçäo de corrente eficiente foram renovadas com o trabalho de Ohkawa [43] sobre geraçăo de corrente por injeçäo de helicidade, $\vec{B} \cdot \vec{A}$, onde $\vec{A}$ é o potencial vetor, $\vec{B}=\vec{\nabla} \times \vec{A}$. Esta idéía foi desenvolvida por Taylor $[44]$, no limite MHD, e um modelo cinético foi apresentado por Elfimov [45]. Em plasmas magnetizados, a corrente de injeçäo de helicidade pode ser mantida por forças ponderomotoras que agem nos elétrons, dependendo da amplitude dos gradientes dos campos de RF. No caso de ondas rápidas [45], foi demonstrado que tambẻm há corrente induzida por gradientes de densidade de equilibrio e temperatura. A força ponderomotora, em um modelo MHD de dois fluidos, para plasmas magnetizados, foi derivada por Klima [46] para geometria plana. O quadro geral destas forças, produzido por ondas de RF em íons e elétrone, foi revisado na Ref, [19] para plasmas cilíndricos, e na Ref.[47] para uma geometria toroidal.

Aqui, é considerado o uso de ondas de Alfvén como um esquema para gerą̧āo de corrente e a criaçäo de ITBs. São propostos dois tipos de ondas de Alfvén para este propusito: ondas Globais de Alfuén (GA ou GAW) e onda Cinética de Alfvén (CA ou KAW), ou onda lenta quase eletrostática de Alfvén (SQAW). Veja detalhes no Capitulo 2 e também nas Refs. $[8,20,11,35]$. Note que as ondas magnetosónicas rápidas podem ser tratadas da mesma maneira que as ondas GA. A deposigăo de energia de KAW e SQAW se dá em faixas radiais pequenas. Assim, é possível induzir a geraçăo de um perfil de corrente oco para configuraçỏes de cisalhamento magnético invertido ${ }^{189}$ e, simultaneamente, criar fluxos de plasma fortemente cisalhados ${ }^{48}$ para suprimir a turbulência do plasma e manter a ITB. O que torna estes tipos de ondas especiais é que podem ser induzidos ambos os tipos de efeitos na mesma regiāo do raio do plasma no tokanak.

Na próxima parte deste capitulo, usando a aproximaçăo de ótica geométrica para ondas de Alfivén, são apresentadas estimativas para as forçás ponderomotoras, forças de viscosidade e de $\mathrm{RF}$, toroidais e poloidais. Na Seção 4.3 , säo mostrados os resultados dos 
cálculos numéricos das forças ponderomotoras en uma coluna cilíndrica de plasma corn comprimento periódico $2 \pi R_{0}$, para simular uma configuraçăo toroidal com raio principal Ro. A geração de corrente por ondas de Alfuén também é analisada. Uma discussano geral sobre geraçã de corrente por ondas de Alvén e fluxos poloidais e toroidais que estäo baseados no equilibrio entre a forç ponderomotora e a viscosidade dos ions, e as conclusões são apresentadas na Seção 4.4.

\subsection{Equaçōes Básicas para Forģas e Fluxo de Plasma}

Seguindo Elfimov at al19, faz-se uma antilise das médias temporais das equaçoes MHD para plasmas com dois fluidos en condição quase estacionária. Equilibrando a força motora de RF nos elétrons e lons, $\left\langle F_{\theta, \zeta}^{(c, i)}>\right.$ contra as forças viscosas $F_{\theta, \zeta}$ (veja Mikhailovskii e Tsypin [49]) nas direçöes poloidal e toroidal, são obtidas as equaçôes

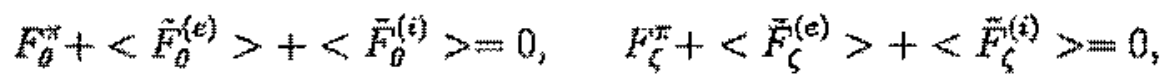

que são as condiçỏes locais para fuxo de plasma çuase estacionário.

\section{4,2,1 Forças Viscosas}

Aqui consideramse as forças viscosas que estäo relacionadas com a modulação do campo magneteico toroidal nas direcoes poloidal e toroidal,

$$
B=B_{0}(1-(r / R) \cos \theta+8 \cos N \varsigma)
$$

onde $r / R$ ê o parãmetro de toroidicidade e f e N sāo os parảmetros de oscilaçào do campo magnético (ripple). Estas forças forarn calculadas por muitos autores (veja, por exemplo, o review de Hirshman e Sigmar [50]) e podern ser apresentadas na forma da Ref.[49]:

$$
F_{\theta}^{\pi}=-m_{i} n_{i} X_{0}\left(U_{i \theta}-\kappa U_{T}\right) \text { e } \quad F_{\zeta}^{\pi}=-m_{i} n_{i} \chi_{\zeta} U_{i \zeta}
$$


onde $\kappa U_{T}$ é a rotação poloidal residual relacionada a uma deriva macroscópica $c T^{u} / e B$, causada pelo gradiente térmico. Neste trabalho é suposto que esta rotaçâo é desprezável en comparaçă com a velocidade poloidal, $U_{i 0}$. O coeficiente de viscosidade poloidal Xo pode ser representado (nos regimes colisional, platear e banana, respectivamente) na forma:

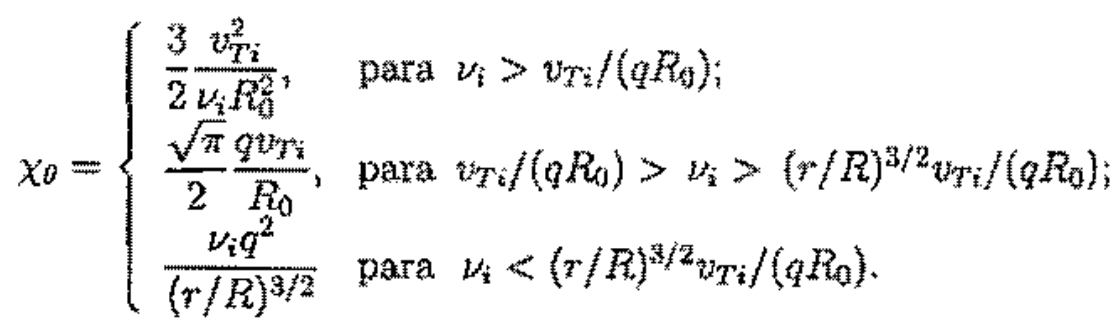

e o coeficiente toroidal, $\chi_{c}$, nos regimes colisional e plateu:

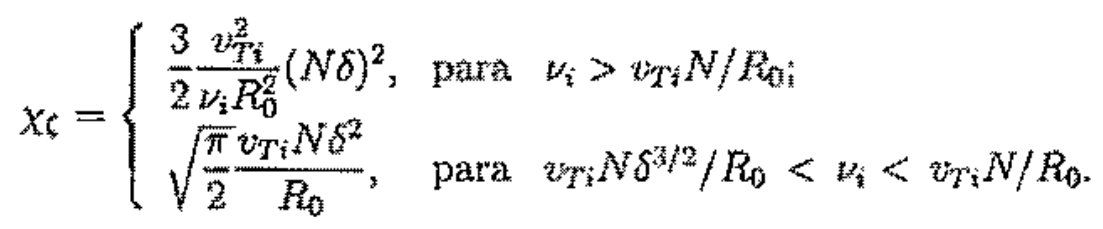

\subsubsection{Forças Ponderomotoras}

O estudo das força ponderomotoras induzidas pelas ondas de Alfvén e rápidas é feito no limite cilindrico, porque as correçoes toroidais $[47]$ são bastante pequenas, $\approx(r / R)^{2}$. As componentes dos campos elétricos de $\mathrm{RF}$ e correntes oscilatórias $\vec{A}=\{\vec{E}(r) ; \vec{f}(r)\}$ sâo tomadas como $\{\overrightarrow{\mathrm{E}}(r) ; \hat{\jmath}(r)\}$ exp $[(\hat{\imath} z+m \theta-\omega t)]$. Por causa do campo magnético helicoidal, o conjunto dos vetores $\vec{A}$ e transformado dos componentes cilindricos $A_{r}, A_{0}$, A para as projeçose normal, binormal paralela, respectivamente,

$$
A_{1}=A_{r}, \quad A_{2} \equiv A_{b}=A_{\theta} h_{\zeta}-A_{\zeta} h_{0}, \quad A_{3} \equiv A_{\|}=A_{\zeta} h_{\zeta}+A_{\theta} h_{0}
$$

através das componentes poloidal e toroidal has do vetor unitário do campo magnético. De acordo com a teoria de efeitos ponderomotores em um plasma de dois-fluidos, as forçats ponderomotoras podem ser expressas calculando-se a média temporal das equaçües MHD 
para dois fluidos:

$$
\frac{\partial}{\partial t}\left(m_{\alpha} n_{\alpha} V_{\theta, \zeta}^{(\alpha)}\right)+\vec{\nabla}\left(m_{\alpha \alpha} n_{\alpha \alpha} \vec{V}^{(\alpha)} \vec{V}_{\theta, S}^{(\alpha)}\right)=\left(e_{\alpha} n_{\alpha} \vec{E}+\frac{1}{c} \vec{j}^{(\alpha)} \times \vec{B}\right)_{\theta, 5}-\vec{\nabla}_{s} \pi_{s \zeta, \theta}^{(\alpha)}
$$

onde $m_{a:} e_{\alpha}$ săo a massa a a carga das parț́culas, e é suposta uma pressăo cinética homogênea ao longo das superfíties magnêticas $\left(\nabla_{3, \zeta} p^{(\alpha)}=0\right)$. Qualquer variável física $\Phi$ (densidade $n_{(\alpha)}$, velocidade $\nabla^{(\alpha)}$, corrente $\vec{j}^{(\alpha)}=e_{\alpha} n_{\alpha} \vec{V}^{(\alpha)}$, carnpos eléctrico $E \mathrm{e}$ magnético B) é escrita como uma soma de uma parte quasenestacionáría (representada

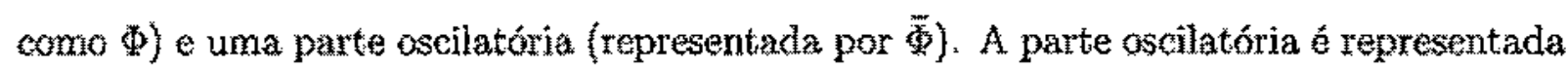

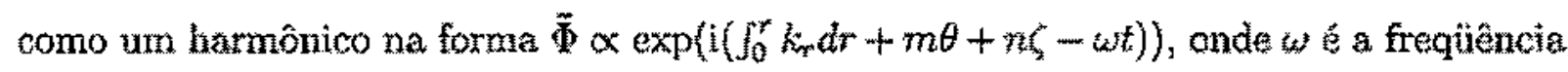
da onda, $m, n$ säo os númetos de onda poloidal e toroidal, e é considerada a aproximagęo eikonal para a dependência radial $\left(\left|k_{r} \Phi\right| \gg|\partial \Phi / \partial r|\right.$, e $\left.\left|k_{r}\right| \gg|m / r|,\left|n / R_{0}\right|\right)$. É usado o limite cilíndrico $\left(r / R_{0} \ll 1\right)$ das coordenadas pseudo-toroidais $(r, 0, \phi)$ com superficies magnéticas conxiais. Seguindo o procedimento habihual de calcular a média da Eq.(79) sobre as oscilaçóes da onda, săo obtidas as expressöes:

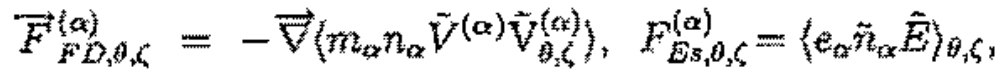

$$
\begin{aligned}
& \left.\vec{F}_{b, \theta, \zeta}^{(\alpha)}=\frac{\tilde{p}^{\alpha} \times \ddot{B}}{c}\right\}_{\theta, \zeta} \text { e } \vec{F}_{V, \theta, \zeta}^{(\alpha)}=-\left\langle\vec{\nabla}_{\left.s, \pi_{s \zeta, \theta}^{(\alpha)}\right\rangle}^{(\alpha)}\right.
\end{aligned}
$$

onde a primeira força é produzida pela tensāo dinảmica do luido (que inclui a tensão de

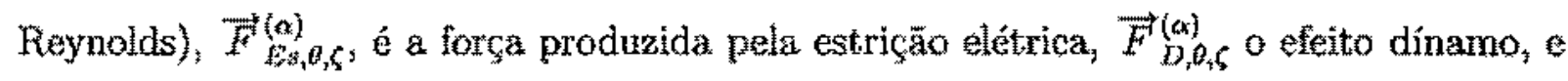
a ultima, $\vec{F}(\alpha, \theta, \beta$, froduzida pela viscosidade. Usando as equaçós de continuidade a de

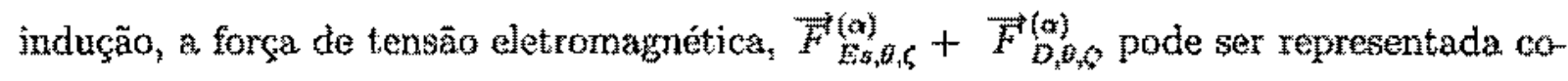
mo a soma $[10,51]$ de uma parte de gradiente, $F_{\partial g}^{(\alpha)}=1 /(2 r w) \operatorname{Im} \nabla_{r}\left(r_{3 r} E_{\theta}^{*}\right)$ e $F_{0,6}^{(\alpha)}=1 /(2 w)$ $\operatorname{Im} \nabla_{r}\left(\vec{j} E_{\zeta}^{*}\right)$, e uma força de tramsterência de momento da onda, $W^{(\alpha)} \vec{k} / \omega$, que é pro-

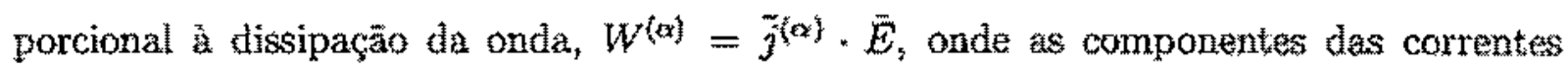


oscilatórias podem ser expressas pela relação,

$$
\tilde{j}_{s}^{(\alpha)}=-\hat{\mathrm{i} \omega} \omega /(4 \pi) \sum_{p} \varepsilon_{s p}^{(\alpha \mathrm{j}} \tilde{E}_{p}
$$

São usadas as expressöes derivadas abaixo (a primeira repetida em detalhe, como exemplo, no Apendice F) para as componentes da força ponderomotora agindo sobre as partículas $\alpha$ (elétrons ou lons) do plasma:

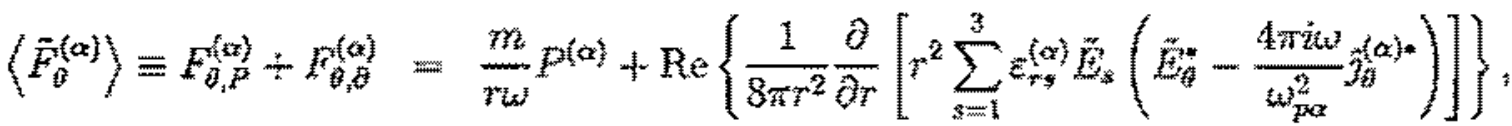

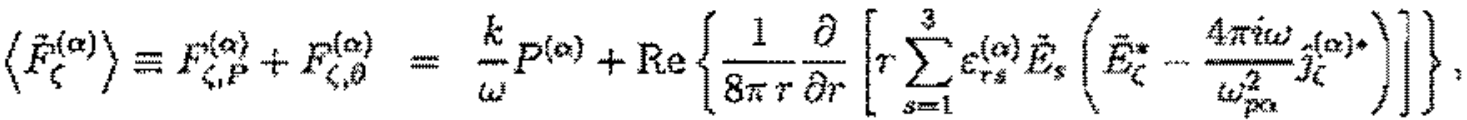

onde os índices $(s=1,2,3)$ säo usados para designar os indices do tensor relacionados com as componentes radial $(r)$, binormal $(b)$, e paralela $(l l)$. A forma do tensor dielétrico $E_{r s}^{(\alpha)}$ usado para os cálculos numéricos mostrados a seguir estả no apêndice $\mathrm{B}$. Na equação anterior, o primeiro termo é a força motriz de transferencicia de momento, $F_{\zeta, O, P}^{(\alpha)}$, que age via dissipaça da onda, e o segundo termo, $F_{\zeta, a}(\alpha)$ é uma mistura da força de gradiente que É relacionada a gradientes dos parametros de equilibrio do plasma com a forga de injeçäo de helicidade (veja discussão na Re. [45]), que csth relacionada ao gradiente da amplitude da onda. Mais adiante, é feita a hipótedse de que a dissipação da onda só se refere aos elétrons, ou seja, $P^{(e)}$ é a média temporal da densidade de potência de $P F$ absorvida pelos elétrons, $\operatorname{Re}\left\langle\overline{\mathbf{E}} \cdot \tilde{\mathbf{j}}^{(e) \star}\right\rangle / 2$.

Para calcular estas forças numericamente para o TCABR, é usado o código cinético toroidal dascrito no capítulo anterior com os dados que seguem. Os valores da transfomada rotacional inversa no eixo magnético, e do campo magnético toroidal no centro do plasma foram tomados como $\sigma_{0}=1,1$ e $B_{0}=10 k G$, respectivamente. $O$ perfil de densidade dos elétrons ê determinado por $n_{e}=n_{0}\left(1-q^{2} / a^{2}\right)+n_{i} \operatorname{com} n_{0}=3 \times 10^{13} \mathrm{~cm}^{-3}$ $n_{n_{\alpha}}=1 \times 10^{12} \mathrm{~cm}^{-3}$. 
Na Fig. 4.1 é apresentada a distribuiçăo da força notora sobre os elétrons, paralela ao campo magnético de equilibrio, normalizada ao valor máximo da força de transferência de momento, $P_{\text {max }}^{(e)}\left|k_{\|}\right| / \omega$. São usadas as condiçöes de ressonăncia GA para $m=-1$ (veja Capitulo 3 e Ref. [52]) com conversão de modo no harmônico vizinho $(m=0)$ em uma onda Cinética de Alfuén, na posiçäo radial $r=0,70$, no contínuo de Altién, para plasmas de hidrogênio. A freqüência do gerador é $f=4,68 M H z$ e os números de onda toroidal e poloidal $(N, M)$ da antena são -4$)-1$, respectivamente. As forças motoras eletrônicas estāo fortemente localizadas ao redor do ponto de conversäo $r_{A}$. Esta torça é principalmente negativa, por causa dos números de onda negativos, mas a geraça de corrente é positiva. Notamse que a velocidade de rotaçâno toroidal pode ser calculada equilibrando-se a força $\sum_{i} F_{\zeta}^{(i)}$ com a perda do momento toroidal $m_{i} n_{i} V_{\zeta}^{(0)} / T_{6}$, onde $\tau_{E}$ t o tempo de relaxamento do momento. É muito forte a diferença mostrada neste gráfico entre as forşas paralelas calculadas com os valores cilindricos e torotedais dos campos. Tal diferença ocorre sempre quando são realizados cấloulos na frequêtucia dos modos globais porque a dissipaçāo destes é maior nu ressonância local do modo vizinho, $m=0$ (ver Capítulo 3).

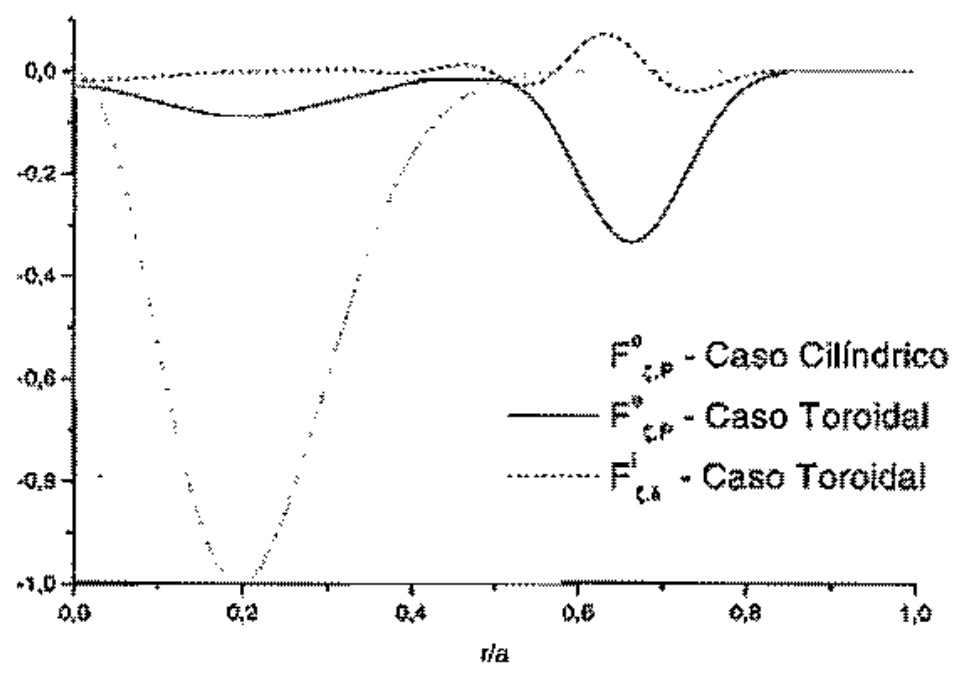

Fíg.4.1 Distribugão radial da componente paralela da forch motora eletrônica $(M=-1, N=-4$ $f=4,7 \mathrm{MHz}$ ) para um plasma de hidrogënio, $n_{\mathrm{de}}=3 \times 10^{13} \mathrm{~cm}^{-3}$, onde as temperaturas centrais 
dos elétronss e dos íons são $T_{100}=500 \mathrm{eV}$ e $T_{40}=300 \mathrm{eV}$, respectivamente. As curvas (pontilhada,

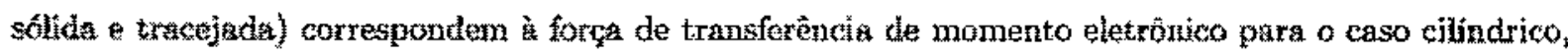

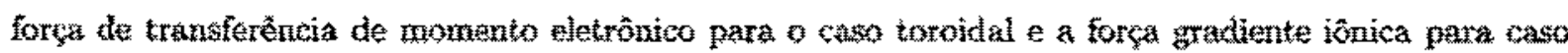
toroidal, respectivamente.

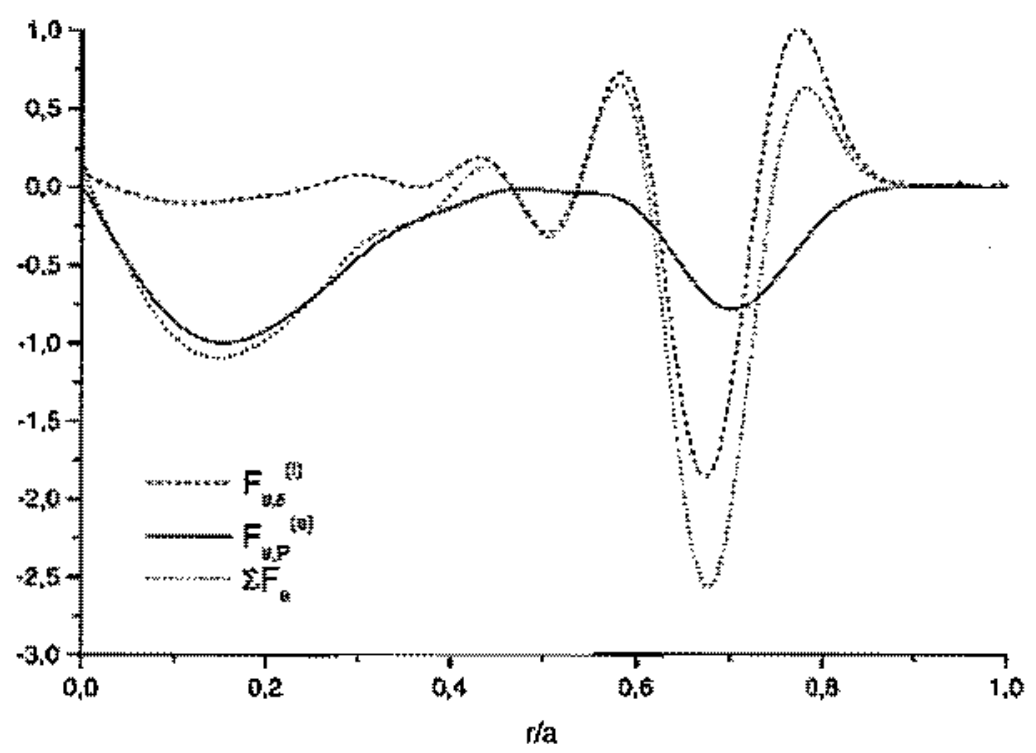

Fìg. 4.2 Distribuiçẫo radial de componente poloidal das forças motrizes para os parämetros da

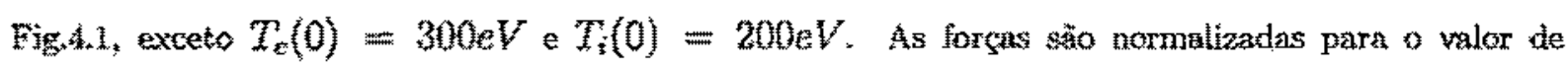

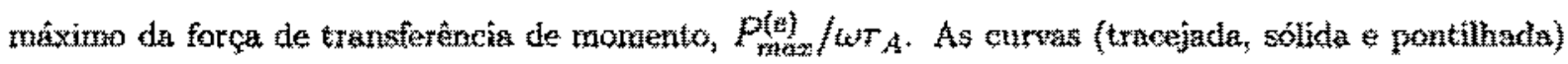
correspondem à forsa gradiente para íons, força de transferência de momento para elétronss a força total sobre o plasma, respectivamente.

Na Fig. 4.2 pode-se observar que o fuxo ionico pode ser dirigido diretamente pela força gradiente que tem sinais opostos nos dois lados da ressonâncla de Alfvén. Note que não bá qualquer dissipação nos fons. Para comparar com os cálculos das forças ponderomotoras geradas por modos GA, é mostrado na Fig. 4.3 a distribuiçăo radial das forças ponderomotoras geradass pelos modos cinéticos, $\mathrm{KAW}$, induzidas através do efeito de conversa de modos en $r=0,5$ para a frequencia $f=5,3 M H z$ temperaturas eletrônicas e iônicas respectivamente iguais a 300 e $200 \mathrm{eV}$, ambos para os casos èlíndrico (um único modo poloidal, veja discussão no Capítulo 2) e toroidal. Aqui é possível verificar 
que, à parte a presenç̧a de uma ressonância local de modo vizinho, as diferenças entre resultados toroidais e cilindricoss năo é grande, diferente da situação mostrada na Fig. 4.1, situada na ressonância global.
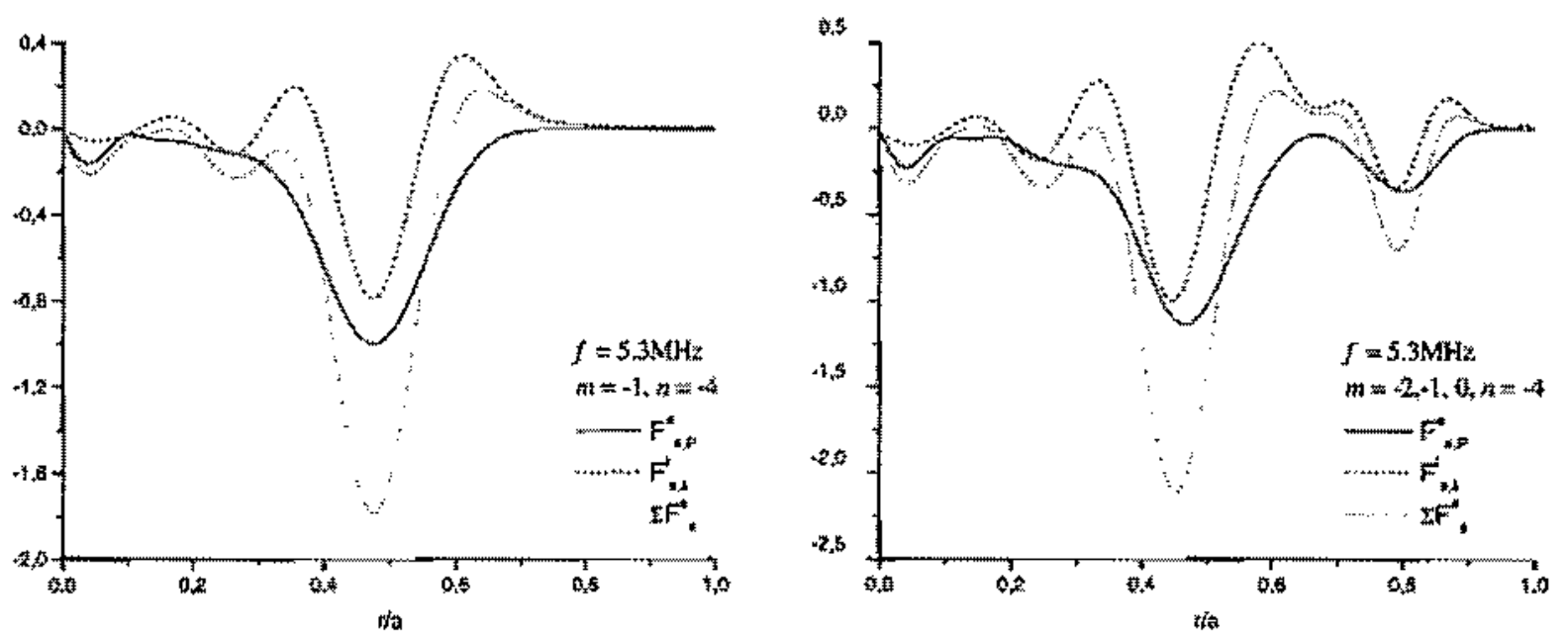

Figara $4.3(\mathrm{a}-\mathrm{b})$ - Forças geradaz na metade do raio to plasma para $f=5,3 M H z$, nos casos cilíndrico (a) e toroidal (b).

Con uma temperatura eletrônica mais baixa, o pico de força fica mais promunciado, o que permitê um cisalhanento maior na velocidade. A Fig. 4.4 mostra o cálculo toroidal com a mesma configuração da Fig. 4.3, mas com temperatura eletrônica de $100 \mathrm{eV}$.

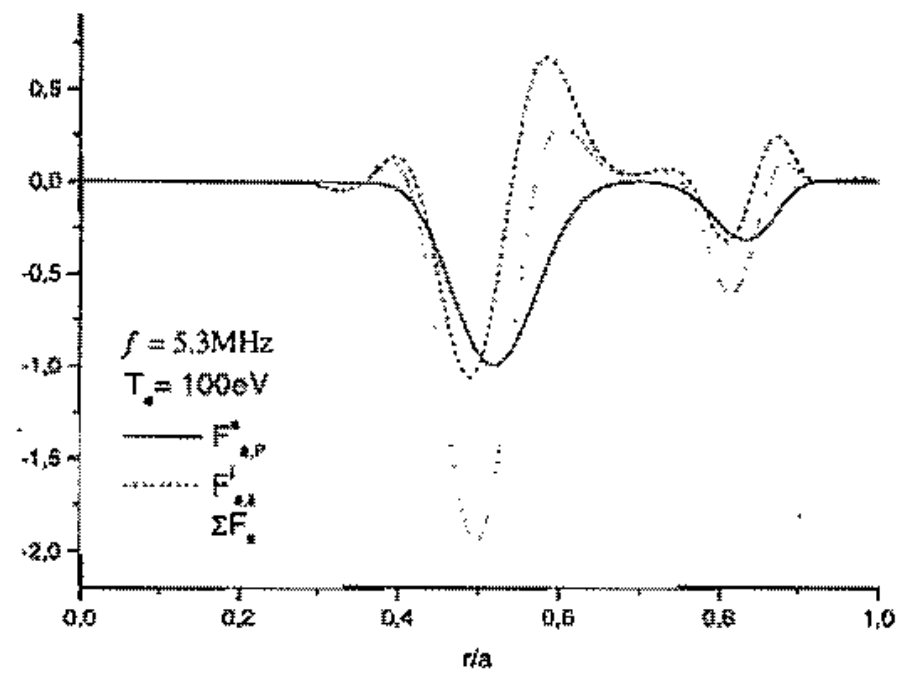

Figura 4,4 - Foryas geradas ma metade do raio do plasma para $f=5,3 M H z_{2}$ pars Temperatura eletrötica de $100 \mathrm{eV}$. 


\subsection{Efeito da Rotação Cisalhada no Transporte Neoclássico}

É possível diminuir o transporte anômalo em plasmas de tokamak através dà rotação cisalhada (através de um campo elétrico radial quase-estacionário cisalhado) ${ }^{(3),[6],[53]}$. Estimativas simples para achar o cisalhamento do campo elétrico radial exigida fornecem $[6],\{38]$

$$
\gamma_{E}=\frac{c B_{\theta} R}{B} \frac{\partial}{\partial r} \frac{E_{r}}{R B_{\theta}} \simeq v_{\theta}(r) / r \geq \gamma_{\text {max }}
$$

onde $\gamma_{\max }$ é a taxa de crescimento das instabilidades mais perigosas na borda do plasma (instabilidades kink e drift, por exemplo). O campo elétrico radial pode ser obtido da equaçẫo de momento dos íns, desprezando a inércia, a friç̧ăo ion-elétron, a viscosidade iônica, e a componentè radial das forças externas $[13]$,

$$
E_{r} \approx \frac{B}{c}\left(-U_{i \theta}+h_{\theta} U_{i \zeta}+U_{i p}\right), \quad U_{i p}=\frac{c}{\epsilon_{i} n_{0} B} \frac{\partial p_{i}}{\partial r},
$$

onde $U_{i p}$ e $V_{i \zeta}$ são as componentes poloidal e toroidal da velocidade dos íons, $V_{i}$, respectivamente, $B$ é o campo magnético, $r$ é o raio menor do tokarnak, $\theta$ e $\zeta$ são os ângulos poloidal e toroidal, respectivamente, $e_{i}$ é a carga dos ions, $M_{i}$ e a massa dos íns.

E suposto que a viscosidade paralela, a qual depende das ondulações do campo magnético ou dos efeitos anômalos, é forte o bastante para suprimir a rotação toroidal. Assim, é considerado o caso $c_{\hat{s}}>U_{i Q}>\left\langle h_{k} U_{k i} ; U_{i p}\right\}$, onde $c_{s}=\sqrt{\left(T_{e}+T_{i}\right) / M_{i}}$ é a velocidade acústica. Então, pode-se aproximar a relação

$$
E_{r} \approx-\frac{B}{c} t_{i \theta}
$$

A velocidade poloidal dos íns e dos elétrons $U_{0}$ pode ser calculada da componente poloidal da equação de momento ${ }^{[5}$

$$
F_{b i}+F_{b e}+F_{b}^{h}=0
$$

que é válida para todos as regimes colisionais. As forças $F_{0}^{\pi}$ e $F_{0}^{h}$ sầ as médias sobre as superficies magnéticas das forças de viscosidade e RF, respectivamente, agindo ao 
longo da direçäo poloidal. Na Eq.(85) é levado em em conta o termo de viscosidade dos elétrons na condiçäo especial de que a viscosidade paralela de íons e elétrons săo da mesma ordem ${ }^{55}, 156 \mid$. As expressốes aproximadas para as forças viscosas poloidais, desprezando-se a rotação residual do plasma ${ }^{[54 !}, \mid$ on?

$$
F_{\theta \alpha}^{\pi} \neq-\mu_{\theta \alpha} U_{\alpha \theta}, \quad \alpha=i, e
$$

onde $\mu_{0 \alpha}$ o coeficiente de viscosidade.

Abaixo é considerada a supressäo do transporte anômalo en um plasma de tokamak fracamente colisional quando as órbitas banana dos fons sāo comprimidas através das ondas Cinéticas de Alfuén ${ }^{[57]}$. Neste caso, o coeficiente de viscosidade $\mu_{i 0}$ depende do

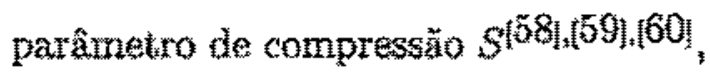

$$
S=1 \cdots \frac{E_{i} B_{\zeta}^{2}}{M_{i} \omega_{\mathrm{c} i}^{2} B_{b}^{2}} \frac{d E_{\mathrm{t}}}{d r}
$$

onde $w_{c j}$ e a frequência ciclotrônica iônica. $O$ coeficiente de viscosidade pode ser expresso pelo parâmetro de compressäo por meio da relay̧ẳo

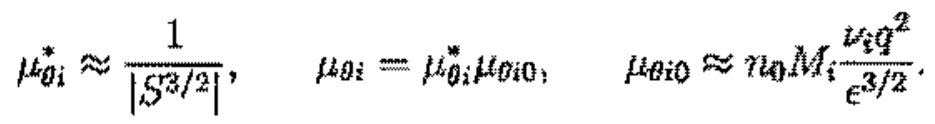

\subsubsection{Equaçöes Diferenciais Para a Viscosidade Iônica}

Com o intuito de azer estimativas aproximadas da dependência da viscosidade dos íns com a potência absorvida da onda Cinética de Alfvén, $W$, a derivada radial do campo elétrico radial quase extacionário $d E_{r} / d r$ é substituida pela expressäo aproximada $E_{r} / \Delta r$,

$$
\frac{d E_{r}}{d r} \approx \frac{E_{r}}{\Delta r}
$$

O parâmetro $\Delta{ }^{*}$ é a mela-largura da curva de dependência radial da componente paralela do campo eletrico de RF El Foi escolhido um modelo de distribuiçăo radial de 
$E_{1}$ na forma $[51]$

$$
E_{1} \approx E_{A} \exp \left[-\frac{1}{2}|x|^{3 / 2}\right], \quad x \approx 2^{5 / 3}(\ln 2)^{2 / 3} \frac{\left(r+r_{0}\right)}{\Delta r}
$$

onde

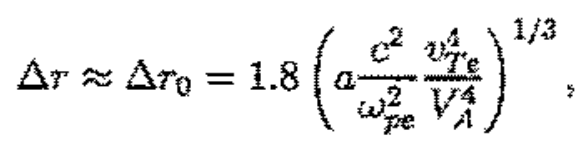

e ro é a coordenada radial do ponto de conversäo. Este modelo hipotético é semelhante à dependência radial real[61] no caso en que a seguinte condiçäo é cumprida:

$$
\frac{M_{e}}{M_{i}}<\beta \ll 1
$$

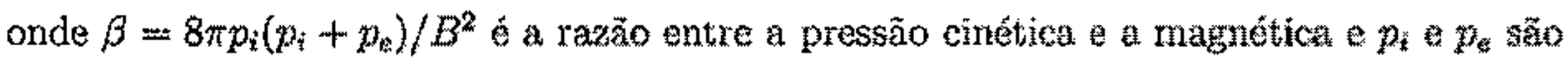
as pressôes ionica e eletrônica, respectivamente. Para ondas Cinéticas de Alvén (KAW), a energia absorvida, $W$, pode ser expressa pela componente paralela, $E_{1}$

$$
W \approx \frac{Q}{8 m} \operatorname{Im} E_{n} \mid E_{U}^{2}
$$

Aqui, $\Omega$ é frequêtrià da KAW, Ime te parte anti-Emitiana da componente pam ralela do tensor de permissividade dielétrica (ver Apendice B). Acsim, como conseqüência das Eqs.(90) e (93), a dependência radial da potência absorvida $W$ é

$$
W \approx W_{A} \exp \left[-|x|^{3 / 2}\right], W_{A}=\frac{\Omega}{8 \pi} \operatorname{Im} \epsilon_{\|}\left|E_{A}\right|^{2} .
$$

No caso das KAW, as forças poloidais $61,, 621$ agindo sobre os lons podem tamberm ser expressas via a potência absorvidat $[54,, 57$,

$$
F_{2}^{b} \approx \frac{m}{m} W
$$

onde m é o número de onda poloidal. Das Eqs (84), (86) e (93), pode-se encontrar o campo

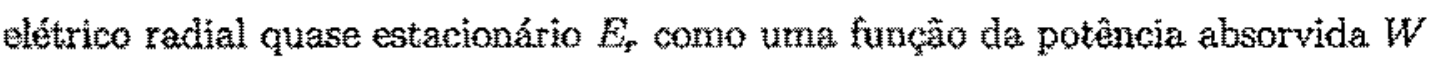

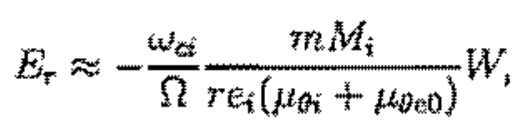


onde

$$
\frac{H_{000}}{\mu_{\theta i 0}} \approx \sqrt{\frac{M_{c}}{M_{i}}}
$$

Usando as Eqs.(87), (88), (92), e (96), mostrada a situaçăo especial em que, na presença da onda Cinética de Alfvên, a dependência radial da wiscosidade dos ions, na regiầ de órbita banana do tokamak, é governada pela seguinte equagäo diferencial:

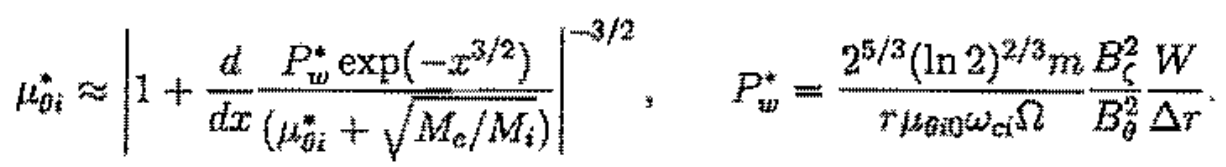

Na fq.(97), fó suposto que todos os parâmetros macroscópicos do plasma sāo funçöes suaves da coordenada ratial, exceto a potencia alssorvida a a viscosidade dos îns.

A meia-largura $\Delta r$ é igual a $\Delta r_{0}$, se $\Delta r_{0}<r_{0}$ e $\Delta r=r_{0}$ se $\Delta r_{0}>r_{0}$ (reja Refs. $[57] \otimes[63]$

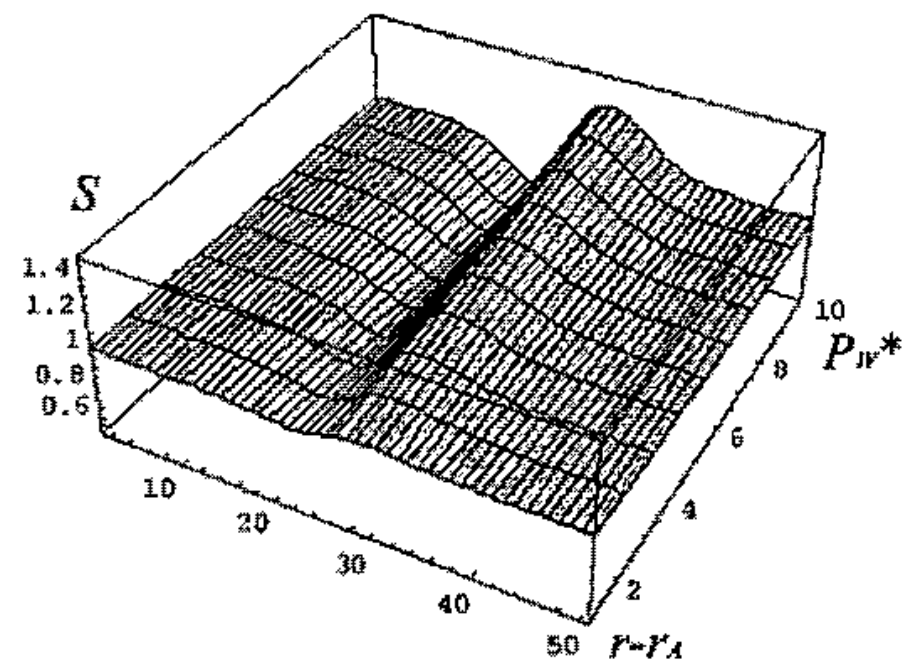

Figura 4.5 - Depandềncia radial da viscosidade iôniea $\mu_{0 i}^{*}$ em função da potência alasorvida $P_{w}^{*}$. 


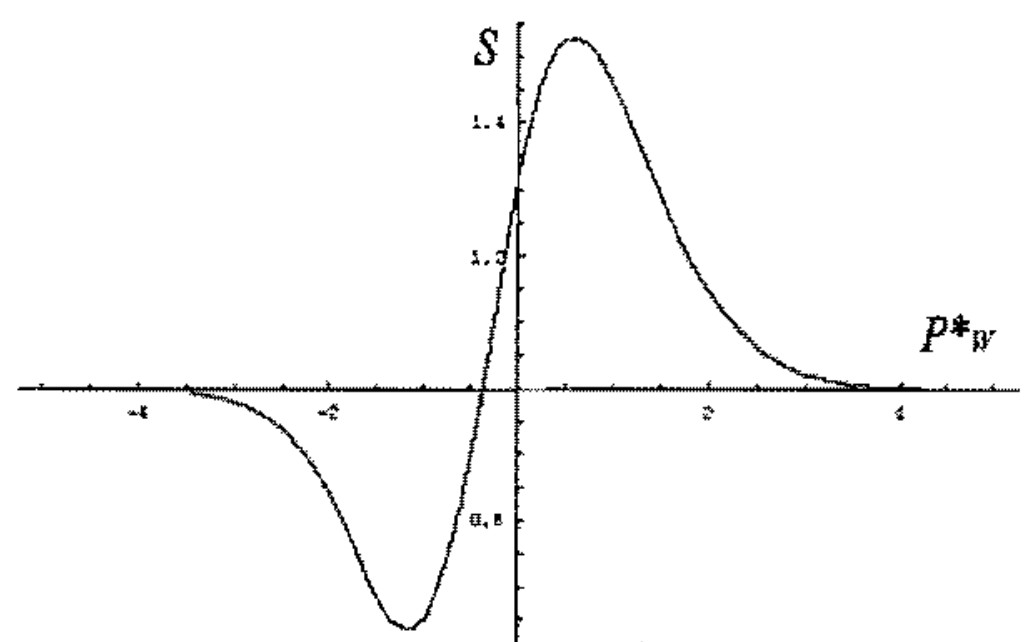

Figura. 4.6-Dependência radial da viscosidade ionica $\mu_{\theta i}^{*}$ para a poténcia absorvida $P_{w}^{*} \ldots, 0,83$

A equação (97) é näo-linear e portanto foi resolvida numericamente. A dependència

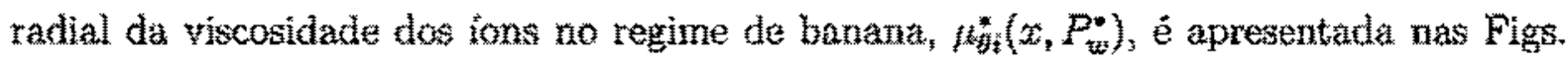
4.5 e 4.6. A soluçăo da equaça diferencial, Eq. (97) é mostrada na Fig.4.5. Percebe-se da Fig.4.6 que a viscosidade de ín normalizada na regiâo de banana de tolkamak é igual à unidade (nenluma mudança com relação ao valor inicial, sem campo elêtrico) longe do ponto de conversăo, onde o poder absorvido de KAW é exponencialmente pequeno. Perto do ponto de conversầ, a viscosidade iónica 6 aumentada ou diminuida dependendo do sinal da potencia absorvida normalizada $P_{w}^{*}$ e da coordenada $x$. So o sinal de $P_{w}^{*}$ mudado, ou seja, se o sinal do número de onda poloidal $m$ é mudado, a Fig.4.6 será a mesma, mas é necessârio transformar $x \rightarrow-x$.

O corte transversal da Fig.4.5 em $p_{w}^{*}=0,83$ e apresentado na Fig. 4.6. Desta figura,

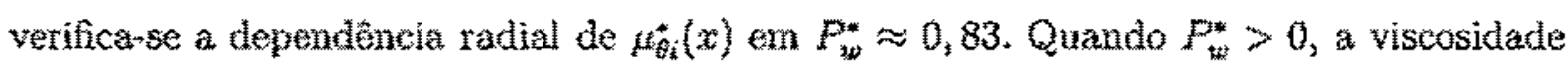
iônica diminut aproximadamente $35 \%$ para $r<\mathrm{r}_{0}$, e aumenta aproximadamente $50 \%$ em $r>r_{0}$. A diminuição da viscosidade na regiño mais interna da coluna de plasma sígnifita uma diminuiģão no transporte neoclássíco do plasma. 


\subsection{Conclusōes}

Uma forma geral para a média temporal da foṛa ponderomotora produzida por ondas de Alfuén am plasmas mametizados foi caloulada. A foŗ̧a ponderomotora caleulada usando a aproximaçāo de dois fuidos inclui contribuiçōes da força te transferência de momento (mais importante no caso dos elétrons) por dissipaçāo da onda e da força gradim ente que e relacionada a gradientes dos parämetros de equilibrio do plasma e amplitude da onda (esta parte mais importante para o caso dos ions). Este feito de gradiente (ou helicidade) pode ser malor que as forças de transferencia de momento da onda.

É ressaltada a importância de correçōes toroidais nos perfís utilizados para calcular as forças ponderomotoras, em especial na presença de modos Clobais de Alfvén.

As torças de gradtentes säo mais importantes para a geraçă de fuxos cisalhados mas são as forças de transferência de momento as mais importantes para a geração de corrente porcue năo apresentam troca de sinal.

Os resultados mostram correntes e fuxos de plasma fortemente localizados gerados pelas ondas de Alfvén cinéticas. Supondo parâmetros que sễo característicos do TCABR, é mostrado que a conversäo de modos cinéticos pode produzir uma configuraçäo de fluxos poloidàs de plasma cisalhados e também um cisalhamento magnético invertido. $O$ valor

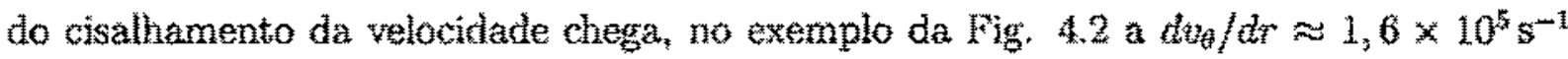
Q pode ser maior que a freqüer̃ncia de deriła $\omega^{*}$, suficiente para suprimir as principais ingtabilidades ${ }^{64}$, no caso do ponto de conversão localizado na metade do raio do plasma com $400 \mathrm{~kW}$ de potencia dissipada pela onda. A posição do ponto de conversấo pode sex controlada pela frepüencia do gerador. Esta configuraçấo pode ser apropriada para a criação das barreiras de transporte internas (ITBs).

Os cálculos mostran que, para temperatura eletrönica reduzida, as forças estäo mais localizadas (e com amplitude levemente maior) na coordenada radial. A dissipaçăo numa 
faixa radial mais estreita 6 benéfica para a criação de LTB's.

Como exemplos de possiveis conseqüèncias da criaçăo de um perfil de campo elétrico radial cisalhado (e consequiente rotaçâo poloidal), sâ̆o mostrados os efeitos de dimimuicāo da viscosidade iônica (e transporte neoclássico).

Importante salientar que os resultados de estimulaçäo de rotaçăo poloidal são importantes tamberm para a contimuaçāo da análise da estabilidade do plasma com relação ao efsito da rotaçăo cisalhada nos modos kink e de Kelvin-Helmholtz, apresentados na dissertaçäo do mestrado 65 e resumido no Apendice G. 


\section{SUMÁRIO E SUGESTÕ̃ES}

Este trabalho tem seu desenvolvimento baseado nos seguintes artigos já publicados:

- A.G. Elfimov, R. M. O. Galvão, I.C. Nascimento and G. Amarante-Segundo Plasma Phys. Contr. Fusion 39 1551. (1997).

- A.G.Elfimov, R.M.O.Galvão, I.C. Nascimento, and G.Amarante-Segundo Plasma Phys. Contr. Fusion 40, 451 (1998).

- G. Amarante-Segundo, A.G. Elfimov, D.W. Ross, R.M.O. Galvão Phys. Plasmas, $6,2437(1.999)$.

- G. Amarante-Segundo, A.G.Elfimov, R.M.O. Galvâa, and I.C.Nascimento - Calculations of Alfven Wave Driving Forces, Plasma Flow and Current Drive In The TCABR Tokamak. Procedings of the $26^{\text {th }}$ EPS Conference on Controlled Futsion and Plasma Physics. 14 - 18 June 1990, Maastricht, The Netherlands Volume 23J, p.1207

- V. S. Tsypin, I. C. Nascimento, R. M. O. Galvão, A. G. Elfimov, G. S. Amarante Segundo and M. Tendler, Phys. Plasmas, 6, 3548 (1999).

- R.M.O. Galvão, A.G. Elfimov, G. Amarante-Segumdo, V. S. Tsypin, L..F. Ruchko, I. C. Nascimento and M. Tendler, Plasma Phys. Contr. Fusion 41 A487 (1999).

Há tambếm o artigo

A.G.Elfmov, G.Amarante Segundo, R.M.O. Galvăo, and I.C. Nagcimento - Ion Lat" mor Radius Effect On RF Ponderomotive Forces And Induced Poloidal Flow In Tokamak Plasmas - aceito para publicaçäo no Physical Review Letters. 


\subsection{Resumo dos resultados}

Num breve sumário do trabalho realizado, as seguintes tarefas foram completadas:

Foi feito um resumo da teoria de aquecimento de plasmas por ondas de Alfvén, para plasmas magnetizados com base no eleito de conversão de modos para plasmas inomogêneos em configuracões cilíndricas e toroidass.

Foi mostrado que o modelo cinético toroidal prediz o divisão do modo $\mathrm{GA}^{120} \mathrm{l}_{\mathrm{em}}$ trểs ressonâncias que são equivalentes às ressonăncias cilíndricas. No caso de umn plasma puro de hidrogênio, com as ressonânelas $\mathrm{GA} m=-1,-2$, o campo de RF é dissipado principalmente na ressonância local de Alfvén dos modos vizinhos perto da periferia do plasma. Estas ressonâncias locais săo equivalentes às ressonâncias do modelo cilindrico com números de modo $m=0,-1$, respectivamente. A ressonância $\mathrm{GA} m=0$ que só aparece no modelo toroidal dissipa sua energia principalmente perto do eixo magnético. Também é mostrado que o modo $N=-6$ é ideal para absorgão das ondas de RF para as condiçöes do TCABR.

O eleito da presença de impurezas minoritárias no plasma foi calculado numericamente; fol mostrada a lacuna no contínuo de Alfvén e fol demonstrada a presença de modos globais de Alfvén.

Com os cálculos $2-D$ é confirmado o princípal resultado dos câleulos unidimensionais do Contínuo Híbrido de Alfvén ${ }^{[35]}$, Além do Contínuo Híbrido de Alfyén cilíndrico para $m \neq 0$, é encontrado o Contínuo Híbrido $m=0$ no modelo toroidal, onde a onda de Alfvén pode dissipar efetivamente com uma impedância de acoplamento da mesma ordem da do modo $m=-1$. Importante ressaltar que os resultados obtidos com o modo $m=0$ são completamente novos.

Usando as condiçôes de aquecimenta do Contínuo Hbrido ${ }^{35}$, é possível levar a cabo - aquecimento por ondas de RF no tokamak TCABR e aplicar os resultados num reator 
tokamak.

Uma forma geral para a média temporal da força ponderomotora produzida por on das de Alfvén em plasmas magnetizados foi calculada. A força ponderomotora calculada usando a aproximação te dois fluidos inclui contribuiçoes da força transterência de momento (mais imporbante no caso dos elétrons) por dissipaçäo da onda e da força gradiente que é relacionada a gradientes dos parâmetros de equilibrio do plasma e a amplitude da onda (esta parte nais importante para o caso dos ions). Este efeito de gradiente (ou helicidade) pode ser maior qua as forças de transferencia de momento da onda.

Os resultados mostram correntes e fuxos de plasma fortemente localizados gerados pelas ondas de Alfvén cinéticas. Supondo parâmetros que săo característicos do TCABR, é mostrado que a conversão de modos cinéticos pode produzir uma configuraçăo de fluxos poloidais de plasma cisalhados, indicando a possibilidade de se suprimir as principais instabilidaded ${ }^{(64)}$ e da criaçäo das barreiras de transporte internas (ITBs).

Os resultados mostram também que é possivel gerar correntes e modiffatur os perfis de correntes no TCABR.

\subsection{Sugestões para continuidade}

Algumas modificaçöes importantes podern ser feitas no código toroidal para almentar a sua eficiencia:

Inchusão de mais modos poloidais acoplados - Como esta escrito agora, o código apenas acopla o modo poloidal principal seus vizinhos mais imediatos ( $m, m \pm 1$ ). Poderse ia adicionar pelo menos mas dois modos ( $m, n \pm 1, m \pm 2)$. Tal modificaçāo implicaria refazer boa parte dos catleulos e praticamente reescrever parte do código. Poderia adicionar confabilidade aos resul tados acerca dos efeitos toroidais, especialmente a freqüencias mais altas. 
Modificação das condiçôes de contorno - As condiçãos de contorno do programa foram feitas para um sistema de antenas helicoidais. Uma melhor adequação a um sistema "multiframe" como o que está sendo instalado no TCABR podera permitir melhores resultados com ondas rảpidas (FW).

Poder-se-ia, também, após o teste da coerência dos resultados com a experiência para situaç̧̋es específicas, nodificar o cởiggo de forma a se calcular a evolų̧ão temporal do sistema. Tal modificaşăo não deveria demandar um esforço muito grande no sentido das mudanças no código, mas requer especial atençāo com a realimentaçăo dos dados de entrada do sistema, de forma a se ter un quadro autoconsistente da evolução do sistema. 
səotpuəd 


\section{A Derivação de coeficientes do tensor dielétrico em}

\section{coordenadas cilíndricas (exemplo)}

Neste apêndice são mostrados os passos pata a derivachăo cinêtica do tensor dielétrico de um plasma magnetizado na forma clíndrich.

Definindo a configuraçầ do plasma cilindrico pelo campo magnêtico toroidal

$$
\overrightarrow{B_{0}}=B_{z} \hat{C}_{z}
$$

mais a Equação de Vlasov

$$
\frac{\partial F_{\alpha}}{\partial t}+\vec{v} \frac{\partial F_{\alpha}}{\partial \vec{r}}+\frac{e_{\alpha}}{m_{\alpha \alpha}}\left\{\vec{B}+\frac{1}{d}[\vec{\theta} \times \vec{B}]\right\} \frac{\partial F_{\alpha x}}{\partial \vec{v}}=S t\left(F_{\alpha}\right)
$$

onde as seguintes quantidades, para as partículas $\alpha(\alpha=$ íns on elétrons) aparecem:

$$
\begin{aligned}
& F_{\alpha}(\vec{r}, \vec{v}, t) \text { - Funçăo de distribuçäo para elétrons e tons. } \\
& \vec{r}(r, \theta, z) \text { - vector do espaço de configuraçäo. } \\
& \vec{v}\left(v_{r}, v_{\theta}, v_{z}\right) \text { - vetor do espaco das velocidades. } \\
& m_{\alpha} \text { - massa da particula. } \\
& e_{\alpha}-\text { carga da partícula. } \\
& \widehat{S} t\left\{F_{\alpha}\right\} \text { - Operador das colisões. } \\
& \vec{E}, \vec{B}-\text { campos elétrico e magnético. }
\end{aligned}
$$

pode-se expandir a Eq, de Vlasov em coordenadas cartesianas

$$
\begin{aligned}
& \frac{\partial F_{\alpha}}{\partial t}+v_{x} \frac{\partial F_{\alpha}}{\partial x}+v_{y} \frac{\partial F_{\alpha}}{\partial y}+v_{z} \frac{\partial F_{\alpha}}{\partial z}+\frac{e_{\alpha x}}{m_{\alpha}}\left\{\left(E_{x}+\frac{v_{z}}{c} B_{z}-\frac{v_{z}}{c} B_{y}\right) \frac{\partial F_{\alpha}}{\partial v_{x}}\right. \\
& \left.\left(E_{y}+\frac{v_{z}}{c} B_{x}-\frac{v_{x}}{c} B_{z}\right) \frac{\partial F_{\alpha}}{\partial v_{y}}+\left(E_{z}+\frac{v_{x}}{c} B_{y}-\frac{v_{z}}{c} B_{x}\right) \frac{\partial F_{\alpha x}}{\partial v_{x}}\right\}=S t\left\{F_{\alpha}\right\}
\end{aligned}
$$

e definir aqui as transformacôes mara coordenadas cilíndricas

$$
X=+\cos \theta, \quad Y=r \sin \theta, \quad Z=z
$$


A seguinte tabela de conversäo se faz necessária:

$$
\begin{aligned}
& d x=\cos \theta d x-\sin \theta d \theta \\
& d y=\sin \theta d r+r \cos \theta d \theta \\
& \tau d \theta=\cos \theta d y-\sin \theta d x \\
& d r^{\prime}=\sin \theta d y+\cos \theta d x \\
& y_{x}=v_{\tau} \cos \theta-y_{\theta} \sin \theta \\
& t_{y}=v_{r} \sin \theta+y_{0} \cos \theta \\
& v_{r}=v_{x} \cos \theta+v_{y} \sin \theta \\
& v_{\theta}=v_{y} \cos \theta-v_{x} \sin \theta \\
& d \nu_{r}=\cos \theta d \nu_{x}+\sin \theta d \nu_{y}-\sin \theta \nu_{z} t \theta+\cos \theta \nu_{y} d \theta \\
& d v_{\theta}=\cos \theta d v_{y}-\sin \theta d v_{x}-\sin \theta v_{y} d \theta-\cos \theta v_{x} d \theta \\
& d v_{x}=d v_{r} \cos \theta-d v_{0} \sin \theta-v_{r} \sin \theta d \theta-v_{y} \cos \theta d \theta= \\
& d v_{r} \cos \theta-d v_{z} \sin \theta-v_{y} d \theta \\
& d v_{y}=d v_{r} \sin \theta+d v_{0} \cos \theta+v_{r} \cos \theta d \theta-v_{g} \sin \theta d \theta= \\
& d v_{r} \sin \theta+d v_{\theta} \cos \theta+v_{2} d \theta
\end{aligned}
$$




$$
\begin{aligned}
& d \theta=-\frac{1}{v_{\theta}}\left(d v_{x} \cos \theta+d v_{y} \sin \theta-d w_{x}\right) \\
& d \theta=\frac{1}{v_{r}}\left(-d v_{x} \sin \theta+d v_{y} \cos \theta-d v_{\theta}\right) \\
& \frac{\partial}{\partial x_{r}}=\frac{\partial r}{\partial x} \frac{\partial}{\partial r}+\frac{\partial \theta}{\partial x} \frac{\partial}{\partial \theta}+\frac{\partial \theta}{\partial x} \frac{\partial u_{r}}{\partial \theta} \frac{\partial}{\partial u_{r}}+\frac{\partial \theta}{\partial x} \frac{\partial v_{\theta}}{\partial \theta} \frac{\partial}{\partial v_{\theta}}= \\
& \cos \theta \frac{\partial}{\partial r}-\frac{\sin \theta}{r} \frac{\partial}{\partial \theta}-\frac{\sin \theta}{r} v_{\theta} \frac{\partial}{\partial v_{r}}+\frac{\sin \theta}{r} v_{r} \frac{\partial}{\partial v_{\theta}} \\
& \frac{\partial}{\partial y}=\frac{\partial r}{\partial y} \frac{\partial}{\partial r}+\frac{\partial \theta}{\partial y} \frac{\partial}{\partial \theta}+\frac{\partial \theta}{\partial y} \frac{\partial v_{r}}{\partial \theta} \frac{\partial}{\partial v_{r}}+\frac{\partial \theta}{\partial y} \frac{\partial v_{\partial}}{\partial \theta} \frac{\partial}{\partial u_{\theta}}= \\
& \sin \theta \frac{\partial}{\partial r}+\frac{\cos \theta}{r} \frac{\partial}{\partial \theta}+\frac{\cos \theta}{r} v_{\theta} \frac{\partial}{\partial v_{r}}-\frac{\cos \theta}{r} v_{r} \frac{\partial}{\partial v_{\theta}} \\
& \frac{\partial}{\partial v_{i}}=\frac{\partial v_{*}}{\partial v_{x}} \frac{\partial}{\partial v_{*}}+\frac{\partial v_{\theta}}{\partial v_{x}} \frac{\partial}{\partial v_{\theta}}+\frac{\partial \theta}{\partial v_{x}} \frac{\partial}{\partial \theta}= \\
& \cos \theta \frac{\partial}{\partial v_{r}}-\sin \theta \frac{\partial}{\partial v_{\theta}}-\frac{\sin \theta}{v_{r}} \frac{\partial}{\partial \theta^{2}} \\
& \frac{\partial}{\partial v_{y}}=\frac{\partial v_{r}}{\partial u_{y}} \frac{\partial}{\partial v_{z}}+\frac{\partial v_{y}}{\partial v_{y}} \frac{\partial}{\partial v_{y}}+\frac{\partial \theta}{\partial v_{y}} \frac{\partial}{\partial \theta}= \\
& \sin \theta \frac{\partial}{\partial v_{r}}+\cos \theta \frac{\partial}{\partial v_{\theta}}+\frac{\cos \theta}{v_{r}} \frac{\partial}{\partial \theta} \text {. }
\end{aligned}
$$

Inserindo as transformaçoes na Eq.de Vlasov, obtém-se

$$
\begin{aligned}
& \frac{\partial F_{\alpha}}{\partial t}+\left(\eta_{r} \cos \theta-v_{\theta} \sin \theta\right)\left(\cos \theta \frac{\partial}{\partial r}-\frac{\sin \theta}{r} \frac{\partial}{\partial \theta}-\frac{\sin \theta}{r} v_{\theta} \frac{\partial}{\partial v_{r}}+\frac{\sin \theta}{r} v_{r} \frac{\partial}{\partial v_{\theta}}\right) F_{\alpha}+ \\
& \left(v_{r} \sin \theta+v_{0} \cos \theta\right)\left(\sin \theta \frac{\partial}{\partial r}+\frac{\cos \theta}{r} \frac{\partial}{\partial \theta}+\frac{\cos \theta}{r} v_{\theta} \frac{\partial}{\partial v_{r}}-\frac{\cos \theta}{r} v_{r} \frac{\partial}{\partial v_{0}}\right) F_{\alpha}+v_{*} \frac{\partial F_{\alpha}}{\partial z}+ \\
& \frac{e_{\alpha}}{m_{\alpha}}\left\{\left(E_{r} \cos \theta-E_{\theta} \sin \theta+\frac{\eta_{r} \sin \theta+\eta_{0} \cos \theta}{c} B_{z}-\frac{v_{z}}{c}\left(B_{r} \sin \theta+B_{\theta} \cos \theta\right)\right)\right. \\
& x\left(\cos \theta \frac{\partial}{\partial v_{r}}-\sin \frac{\partial}{\partial v_{\theta}}\right) \\
& +\left(E_{r} \sin \theta+E_{\theta} \cos \theta+\frac{v_{z}}{c}\left(B_{r} \cos \theta-B_{\theta} \sin \theta\right)-\frac{v_{r} \cos \theta-v_{\theta} \sin \theta}{c} B_{z}\right) \\
& x\left(\sin \theta \frac{\partial}{\partial v_{r}}+\cos \theta \frac{\partial}{\partial v_{\theta}}\right)+ \\
& \left(E_{z}+\frac{v_{r} \cos \theta-v_{\theta} \sin \theta}{c}\left(B_{r} \sin \theta+B_{\theta} \cos \theta\right)-\frac{v_{r} \sin \theta+v_{\theta} \cos \theta}{c}\left(B_{r} \cos \theta-B_{\theta} \sin \theta\right)\right) \\
& \left.\times \quad \frac{\partial}{\partial q_{*}}\right\} F_{\alpha}=\widehat{S} t\left\{F_{z}\right\}
\end{aligned}
$$


e passando, definitivamente para coordenadas cilindricas,

$$
\begin{aligned}
& \frac{\partial F_{\alpha}}{\partial t}+v_{r} \frac{\partial F_{\alpha}}{\partial r}+\frac{v_{\theta}}{r} \frac{\partial F_{\alpha}}{\partial \theta}+\frac{v_{\theta}}{r}\left(v_{\theta} \frac{\partial}{\partial v_{r}}-v_{r} \frac{\partial}{\partial v_{\theta}}\right) F_{\alpha}+v_{z} \frac{\partial F_{\alpha}}{\partial z}+
\end{aligned}
$$

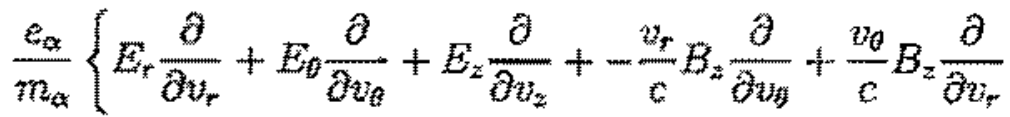

$$
\begin{aligned}
& \left.+\frac{v_{z}}{c} B_{r} \frac{\partial}{\partial \nu_{\theta}}-\frac{v_{\theta}}{c} B_{r} \frac{\partial}{\partial \nu_{z}}+\frac{v_{r}}{c} B_{0} \frac{\partial}{\partial \nu_{z}}-\frac{v_{z}}{c} B_{0} \frac{\partial}{\partial v_{*}}\right\} F_{\alpha}=\widehat{S} z\left\{F_{\alpha}\right\} \\
& \frac{\partial F_{\alpha}}{\partial t}+v_{r} \frac{\partial F_{\alpha}}{\partial r}+\frac{v_{0}}{r^{r}} \frac{\partial F_{\alpha}}{\partial \theta}+\frac{v_{\theta}}{r}\left(v_{\theta} \frac{\partial}{\partial v_{r}}-v_{r} \frac{\partial}{\partial v_{0}}\right) F_{\alpha}+v_{z} \frac{\partial F_{\alpha}}{\partial z}+ \\
& \frac{e_{\alpha}}{m_{\alpha}}\left\{\left(E_{\mathrm{z}}+\frac{1}{c_{c}}\left[B_{z} v_{\theta}-B_{\theta} v_{z}\right]\right) \frac{\partial}{\partial v_{r}}+\left(E_{p}+\frac{1}{c}\left[B_{\mathrm{r}} v_{z}-B_{z} v_{r}\right]\right) \frac{\partial}{\partial v_{\theta}}\right.
\end{aligned}
$$

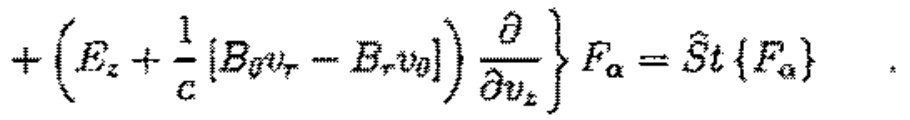

Agora, definindo um sistema de coordenadas baseado no campo magnetico de equilibrio, com as direções paralela e perpendicular a $\vec{B}_{0}$, e com o ấngulo $\sigma$ :

$$
\begin{aligned}
& v_{r}=v_{L} \cos \alpha_{i} \\
& v_{9}=v_{1} \sin \theta \\
& v_{z}=v_{\| i} \\
& d v_{r}=\cos \sigma d v_{1}-v_{1} \sin \sigma d \sigma \\
& d v_{\theta}=\sin \sigma d v_{1}+v_{1} \cos \sigma d \sigma \\
& d v_{1}=d v_{0} \sin \sigma+d v_{r} \cos \sigma_{;} \\
& v_{1} d \sigma=\cos \alpha d z_{0}-\sin \alpha d t_{t} \\
& \frac{\partial}{\partial v_{r}}=\frac{\partial v_{1}}{\partial v_{r}} \frac{\partial}{\partial v_{1}}+\frac{\partial \sigma}{\partial v_{r}} \frac{\partial}{\partial \sigma}=\cos \sigma \frac{\partial}{\partial v_{1}}-\frac{\sin \sigma}{\partial v_{1}} \frac{\partial}{\partial t} \\
& \frac{\partial}{\partial v_{\theta}}=\frac{\partial v_{1}}{\partial v_{\theta}} \frac{\partial}{\partial v_{1}}+\frac{\partial \sigma}{\partial v_{0}} \frac{\partial}{\partial \sigma}=\sin \sigma \frac{\partial}{\partial v_{1}}+\frac{\cos \alpha}{v_{1}} \frac{\partial}{\partial \sigma^{\circ}} \text {. }
\end{aligned}
$$


Substituindo na Equação de Vlasov cilindrica,

$$
\begin{aligned}
& \frac{\partial F_{\alpha}}{\partial t}+v_{\perp} \cos \sigma \frac{\partial F_{\alpha}}{\partial t}+\frac{v_{1}}{r} \sin \sigma \frac{\partial F_{\alpha}}{\partial \theta}+v_{1} \frac{\partial F_{\alpha}}{\partial z} \\
& +\frac{v_{\perp}^{2} \sin \sigma}{r}\left[\sin \sigma\left(\cos \sigma \frac{\partial}{\partial v_{\perp}}-\frac{\sin \sigma}{v_{\perp}} \frac{\partial}{\partial \sigma}\right)-\cos \sigma\left(\sin \sigma \frac{\partial}{\partial v_{\perp}}+\frac{\cos \sigma}{v_{\perp}} \frac{\partial}{\partial \sigma}\right)\right] F_{c x} \\
& +\frac{e_{\alpha \alpha}}{m_{\alpha}}\left\{\left(E_{\mathrm{r}}+\frac{1}{c}\left[B_{1} \omega_{L} \sin \sigma-B_{\theta} v_{\|}\right]\right)\left(\cos \sigma \frac{\partial}{\partial \nu_{\perp}}-\frac{\sin \sigma}{v_{\perp}} \frac{\partial}{\partial \sigma}\right)\right. \\
& +\left(E_{0}+\frac{1}{c}\left[B_{r} v_{\|}-B_{\|} v_{\perp} \cos \sigma\right]\right)\left(\sin \sigma \frac{\partial}{\partial v_{\perp}}+\frac{\cos \sigma}{v_{\perp}} \frac{\partial}{\partial \sigma}\right) \\
& \left.+\left(E_{\|}+\frac{1}{c}\left[B_{\hat{\theta}} y_{\perp} \cos \sigma-B_{r} v_{\perp} \sin \sigma\right]\right) \frac{\partial}{\partial v_{\|}}\right\} F_{\alpha}=S t\left(F_{\alpha \alpha}\right\}, \\
& \frac{\partial F_{\alpha}}{\partial t}+v_{\perp} \cos \sigma \frac{\partial F_{\alpha}}{\partial r}+\frac{v_{\perp}}{r} \sin \sigma \frac{\partial F_{\alpha x}}{\partial \theta}+v_{\|} \frac{\partial F_{\alpha}}{\partial z} \\
& +\frac{v_{\perp}^{2} \sin \sigma}{r}\left[\sin \sigma\left(\cos \sigma \frac{\partial}{\partial v_{\perp}}-\frac{\sin \sigma}{v_{\perp}} \frac{\partial}{\partial \sigma}\right)-\cos \sigma\left(\sin \sigma \frac{\partial}{\partial v_{\perp}}+\frac{\cos \sigma}{v_{\perp}} \frac{\partial}{\partial \sigma}\right)\right] F_{\alpha x} \\
& +\frac{e_{\alpha}}{m_{\alpha x}}\left\{\left(E_{\mathrm{r}}+\frac{1}{c}\left[B_{\|} v_{\perp} \sin \sigma-B_{\theta} v_{1}\right]\right)\left(\cos \sigma \frac{\partial}{\partial v_{\alpha}}-\frac{\sin \theta}{v_{\perp}} \frac{\partial}{\partial \sigma}\right)\right. \\
& +\left(E_{\theta}+\frac{1}{c}\left[B_{\tau} v_{\|}-B_{\|} v_{\perp} \cos \sigma\right]\right)\left(\sin \sigma \frac{\partial}{\partial u_{\perp}}+\frac{\cos \sigma}{v_{\perp}} \frac{\partial}{\partial \sigma}\right) \\
& +\left(E_{\|}+\frac{1}{c}\left[B_{\theta} v_{\perp} \cos \sigma-B_{r} v_{\perp} \sin \sigma\right) \frac{\partial}{\partial v_{i}}\right\} F_{\alpha}=\hat{S} t\left\{F_{\alpha}\right\}
\end{aligned}
$$

$$
\begin{aligned}
& \frac{\partial F_{\alpha}}{\partial t}+v_{\perp} \cos \sigma \frac{\partial F_{\alpha z}}{\partial r}+\frac{v_{1}}{\eta} \sin \sigma \frac{\partial F_{\alpha}}{\partial \theta}+v_{\|} \frac{\partial F_{\alpha}}{\partial z}-\frac{v_{\alpha} \sin \sigma}{r} \frac{\partial}{\partial t} F_{\alpha \alpha} \\
& +\frac{e_{\alpha}}{m_{\alpha \alpha}}\left\{\left[\left(E_{\tau}-\frac{1}{c} B_{\partial} v_{\|}\right) \cos \sigma+\left(E_{\theta}+\frac{1}{c} B_{r} v_{l}\right) \sin \sigma\right] \frac{\partial}{\partial v_{\perp}}\right. \\
& +\left[\left(E_{0}+\frac{v_{1}}{c} B_{r}\right) \frac{\cos \sigma}{v_{\perp}}-\left(E_{r}-\frac{v_{1}}{c} B_{\theta}\right) \frac{\sin \sigma}{v_{\perp}}-\frac{1}{c} B_{\|}\right] \frac{\partial}{\partial \sigma} \\
& \left.+\left(B_{\|}+\frac{v_{\perp}}{c}\left\{B_{\theta} \cos \sigma-B_{r} \sin \sigma\right]\right) \frac{\partial}{\partial t_{\|}}\right\} F_{\alpha}=S i\left\{F_{\alpha}\right\} \text {, }
\end{aligned}
$$

substituindo-se a freqüểnca ciclotrônica das partículas, $\omega_{\mathrm{ca}} \simeq \frac{e_{\alpha}}{m_{\alpha} c} B_{\|}$, obtêm-se

$$
\begin{aligned}
\frac{\partial F_{\alpha}}{\partial t}+v_{\perp} \cos \sigma \frac{\partial F_{\alpha}}{\partial r} & +\frac{v_{\perp}}{r} \sin \sigma \frac{\partial F_{\alpha}}{\partial \theta}+v_{\|} \frac{\partial F_{\alpha}}{\partial z}-\left(\omega_{\mathrm{c}}+\frac{v_{\perp}}{r} \sin \sigma\right) \frac{\partial F_{\alpha}}{\partial \sigma} \\
+\frac{e_{\alpha}}{m_{z x}}\left\{\left[\left(E_{r}-\frac{v_{\|}}{c} B_{\theta}\right) \cos \sigma\right.\right. & \left.+\left(E_{\theta}+\frac{v_{\|}}{c} B_{r}\right) \sin \sigma\right] \frac{\partial F_{\alpha \alpha}}{\partial v_{\perp}}+\left[\frac{v_{\perp}}{c}\left(B_{\theta} \cos \sigma-B_{\mathrm{r}} \sin \sigma\right)+E_{3}\right] \frac{\partial F_{\alpha}}{\partial v_{\|}} \\
& \left.+\left[\left(E_{\theta}+\frac{v_{\|}}{c} B_{r}\right) \cos \sigma-\left(E_{r}-\frac{v_{\|}}{c} B_{\theta}\right) \sin \sigma\right] \frac{\partial F_{\alpha}}{v_{\perp} \partial \sigma}\right\}=\hat{S} t\left\{F_{\alpha}\right\} .
\end{aligned}
$$


Fazendo uma expansão para a perturbaçäo em primeira ordem da funçăo de distribuiçăo das particulas:

$$
\tilde{f}=\left\{f_{0}+\sum_{t \neq 0}\left[f_{r_{1} l} \cos (l \sigma)+f_{b_{i}} \sin (l \sigma)\right]\right\} \exp i(m \theta+k z-\Omega)
$$

e substituindo a expressä́o acima pela expansăo de onda plana, obtém-se a expressäo total de $F$, em primeira ordem no espaco de configuraçăo e de velocidades:

$$
F=F_{M}+F_{b} \sin \sigma+F_{r} \cos \sigma+\left(f_{0}+f_{t} \cos \sigma+f_{b} \sin \sigma\right) \exp \left(m \theta+h_{z} z-\Omega t\right)
$$

Substituindo essa última na Eq.99 independente do tempo e sem os termos de campo perturbado,

$$
\begin{aligned}
& v_{1} \cos \sigma \frac{\partial F_{0 \alpha}}{\partial r}+v_{1} \cos ^{2} \alpha \frac{\partial F_{r \alpha}}{\partial r}-\omega_{c \alpha} \frac{\partial F_{\alpha}}{\partial \sigma}-\frac{v_{1}}{r} \sin \alpha \frac{\partial F_{\alpha}}{\partial \sigma}=0
\end{aligned}
$$

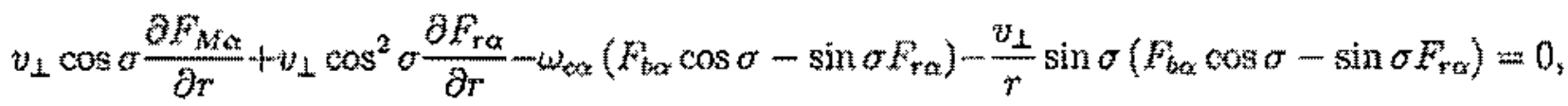

$$
\begin{aligned}
& \psi_{\perp} \frac{\partial F_{M \alpha}}{\partial r}+\nu_{\perp} \cos \alpha \frac{\partial F_{r \alpha}}{\partial r}-\omega_{c \alpha}\left(F_{b \alpha}-\frac{\sin \sigma}{\cos \theta} F_{r \alpha}\right)-\frac{\partial_{\perp}}{r} \sin \theta\left(F_{b \alpha}-\frac{\sin \sigma}{\cos \sigma} F_{z \alpha \alpha}\right)=0,
\end{aligned}
$$

que só è válida com $F_{r a}=0$. Eliminando os termos de primeina ordem em $\sigma$,

$$
v_{1} \frac{\partial F_{M a x}}{\partial r}=\omega_{c} F_{b a x}
$$

Assim é demonstrado que a ordem zero paza a aproximaçăo de onda é

$$
F_{0 \alpha x}=F_{M \alpha}+F_{b \alpha} \sin \sigma_{2}
$$

onde

$$
F_{N \alpha}=\frac{N_{0 \alpha}}{\left(2 \pi v_{T \alpha}^{2}\right)^{3 / 2}} \exp \left[-\frac{v_{1}^{2}+\left(v_{1}-v_{0 \alpha}\right)^{2}}{2 v_{t \alpha}^{2}}\right], \quad F_{b \alpha}=\frac{v_{1}}{\omega_{c \alpha}} \frac{d F_{U \alpha}}{d r}
$$

Levarido-se em conta

$$
\begin{aligned}
& \cos ^{2} \theta=\frac{1}{2}+\frac{1}{2} \cos 2 \sigma \\
& \sin ^{2} \theta=\frac{1}{2}-\frac{1}{2} \cos 2 \sigma
\end{aligned}
$$


e fazento a hipótese do operador de Krook,

$$
\widehat{S} t\left\{F_{\mathrm{a}}\right\}=\nu_{e} F_{\mathrm{e}}
$$

obtém-se, da Eq.(99) a seguinte expressão para os termos indepententes de o:

$$
\begin{aligned}
& -i n f_{0}+v_{\perp} \cos ^{2} \alpha \frac{\partial f_{r}}{\partial r}+i m \frac{y_{1}}{r} \sin ^{2} \sigma f_{t}+i k_{\|} v_{\|} f_{0}+\frac{w_{1}}{r} \sin ^{2} \sigma f_{r}+
\end{aligned}
$$

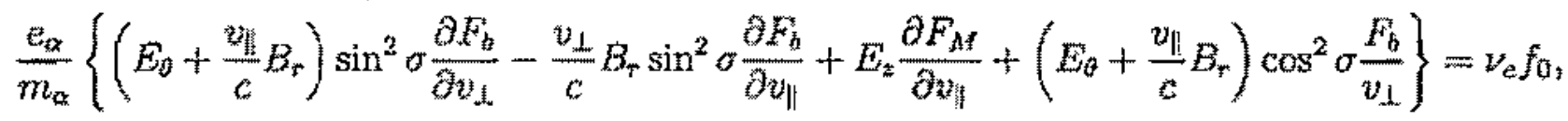

ot mellior,

$$
\begin{aligned}
& \left(-i \rho+i h_{n} v_{n}-\nu_{e}\right) f_{0}+\frac{v_{\perp}}{2} \frac{\partial f_{r}}{\partial r}+\frac{v_{1}}{2 r} f_{r}+i m \frac{v_{\perp}}{2 r} f_{b}+
\end{aligned}
$$

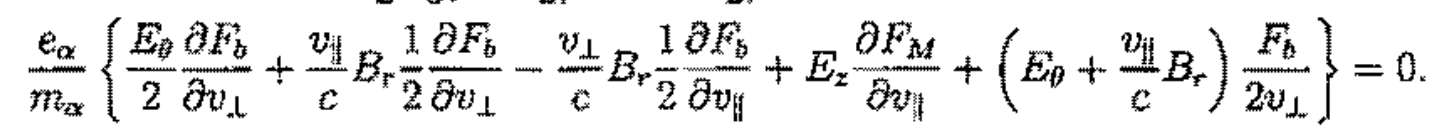

Usando a dentidade

$$
v_{i} \frac{\partial F_{b}}{\partial v_{1}}=v \frac{\partial F_{b}}{\partial v_{1}}
$$

chega-se, finalmente, a axpressão

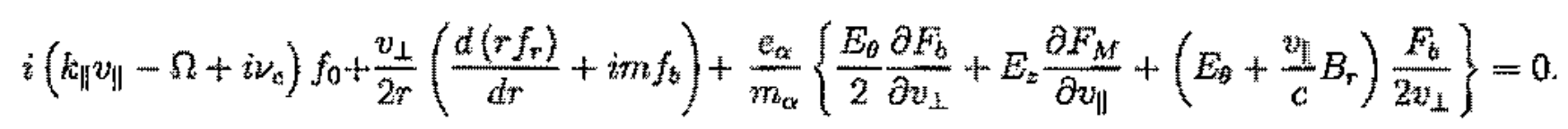

Fazendo a transformaçäo

$$
w \rightarrow n-i, k e f
$$

e seguindo os mesmos procedimentos para as componentes proporcionais a $\cos \theta$ esin $\sigma$ da la (99), obtém-se o conjunto de equaçôes das quantidades perturbata

$$
\begin{aligned}
& i\left(k_{1} v_{1}-w\right) f_{0}+\frac{u_{\perp}}{2}\left(\frac{d f_{r}}{d r}+\frac{F_{r}}{r}+i \frac{m}{r} f_{b}\right) \\
= & -\frac{\varepsilon_{\alpha}}{m_{\alpha}}\left[E_{3} \frac{\partial F_{M \alpha}}{\partial v_{1}}+\frac{E_{2}}{\omega_{c}} \frac{\partial}{\partial r}\left(\frac{F_{M \alpha}}{2}+\frac{v_{\perp}}{2} \frac{\partial F_{M \alpha}}{\partial v_{\perp}}\right)+\frac{v_{\|}}{2 c v_{\perp}} B_{1} F_{a}\right]
\end{aligned}
$$




$$
\begin{aligned}
& \omega_{c} f_{b}=-\left(\omega-k_{\|} v_{\|}\right) f_{r}+w_{\perp} \frac{\partial f_{0}}{\partial r}+\frac{e_{\alpha}}{m_{\alpha}}\left[E_{r} \frac{\partial F_{M \alpha}}{\partial v_{\perp}}-\frac{B_{z}}{c} F_{b}\right]
\end{aligned}
$$

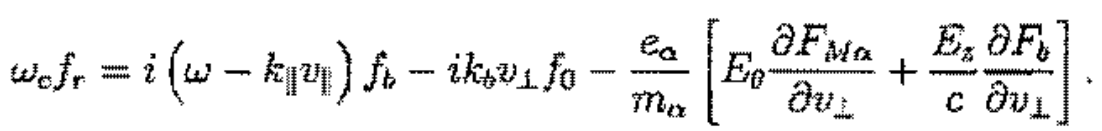

Incluindo(104) em, (103), obtém-se

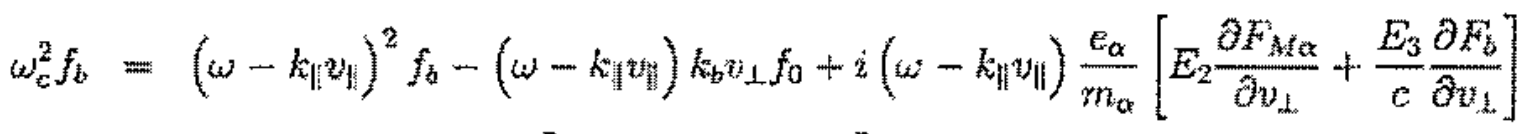

$$
\begin{aligned}
& +w_{c} v_{1} \frac{\partial f_{0}}{\partial r}+w_{c} \frac{e_{\alpha}}{m_{\alpha_{\alpha}}}\left[E_{1} \frac{\partial F_{M \alpha}}{\partial v_{1}}-\frac{B_{3}}{c} F_{t}\right]
\end{aligned}
$$

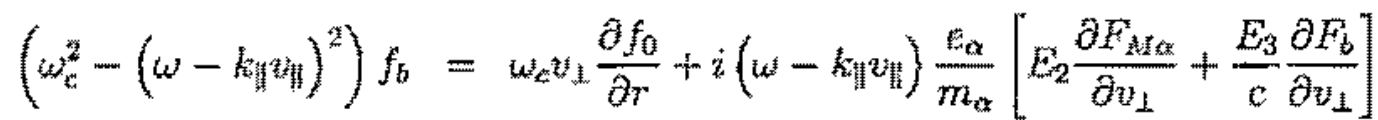

$$
\begin{aligned}
& -\left(\omega-k_{\|} v_{1}\right) k_{b} v_{\perp} f_{0}+\frac{\omega_{c} E_{\alpha}}{m_{0}}\left[E_{1} \frac{\partial F_{M a}}{\partial v_{\perp}}-\frac{B_{B}}{\epsilon} F_{b}\right] \text {. }
\end{aligned}
$$

Incluindo (103) em (104), obtém-se

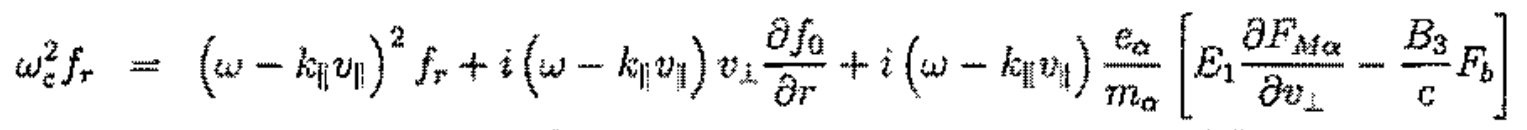

$$
\begin{aligned}
& -i k_{b} v_{L} f w_{c}-\frac{\varepsilon_{\alpha} \omega_{c}}{m_{\alpha}}\left[E_{2} \frac{\partial F_{M o}}{\partial v_{\perp}}+\frac{E_{3}}{c} \frac{\partial F_{b}}{\partial v_{\perp}}-\frac{B_{1}}{c}\left(\frac{v_{\perp} v_{0}}{v_{T \alpha}^{2}} F_{M \alpha}\right)\right]
\end{aligned}
$$

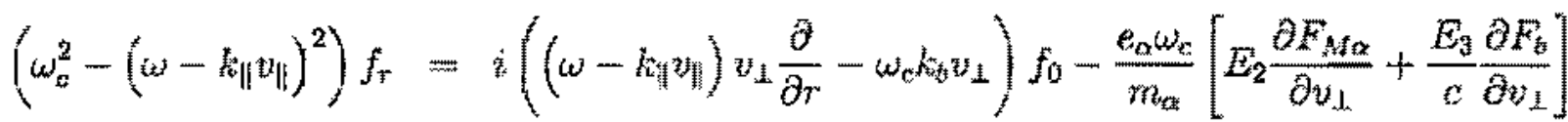

$$
\begin{aligned}
& +3\left(w-k_{i} v_{\|}\right) \frac{e_{\alpha}}{m_{a}}\left[E_{i} \frac{\partial F_{M a}}{\partial v_{\perp}}-\frac{B_{a}}{c} F_{b}\right] \text {. }
\end{aligned}
$$

Estas equações podem ser subatituidas nas equaçōes para a densidade de corrente perturbada

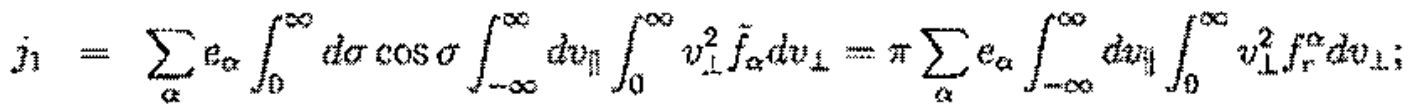

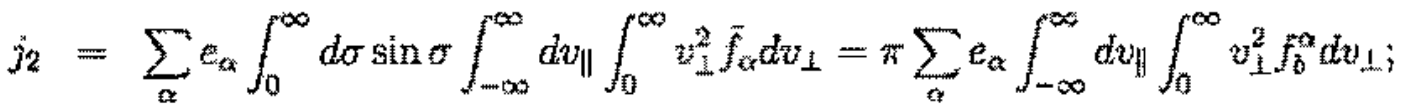

$$
\begin{aligned}
& \hat{j}_{3}=\sum_{\alpha} e_{\alpha} \int_{0}^{\infty} v_{1} d \sigma \int_{-\infty}^{\infty} d v_{1} \int_{0}^{\infty} v_{\perp} \hat{f}_{\alpha} d v_{\perp}=2 \pi \sum_{\alpha} e_{\alpha} \int_{-\infty}^{\infty} v_{1} d v_{\|} \int_{0}^{\infty} v_{\perp} f_{0}^{\alpha} d v_{\perp}
\end{aligned}
$$


para, junto com as Equaçōes de Maxwell, compor as expressôes finais do tensor dieletrico. Empregando a relação entre a corrente oscilatória e o campo elétrico,

$$
4 \pi j_{i}=-i n\left(\delta_{i j}-E_{i j}\right) E_{j,}
$$

onde $\delta_{i j}$ a delta de Kroeneker, chega-ge às componentes do tensor dielétrico

$$
\begin{aligned}
& \hat{\epsilon}_{11}=\sum_{\alpha} \frac{w_{p_{\alpha}}^{2}}{w_{c \alpha \alpha}^{2}}\left[\left(1-\frac{w^{2}}{w_{\alpha \alpha}^{2}}\right)^{-1}+2 \frac{k_{b}^{2} v_{\alpha \alpha}^{2}}{w^{2}}\left(\Lambda_{\alpha}-1\right)\right]
\end{aligned}
$$

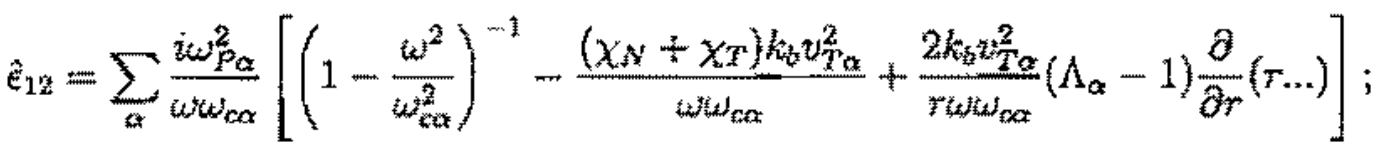

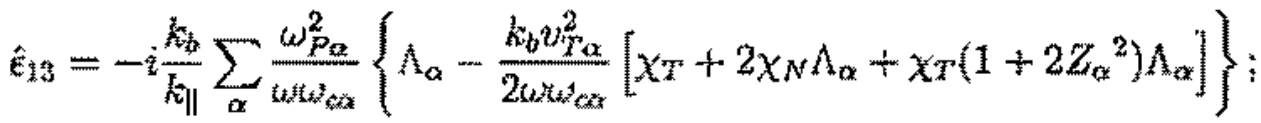

$$
\begin{aligned}
& \hat{k}_{21}=-\varepsilon_{12}-i \sum_{\alpha} \frac{\omega_{P_{\alpha}}^{2}}{\omega^{2}} \frac{k_{b} v_{T_{\alpha}}^{2}}{\psi_{\alpha_{\alpha}}^{2}}\left\{\frac{4}{q^{*}}\left(\Lambda_{\alpha}-1\right)-2 \chi_{N} \Lambda_{\alpha}-\chi_{r}\left[\left(1+2 Z_{\alpha}{ }^{2}\right) \Lambda_{\alpha}-1\right]\right\}
\end{aligned}
$$

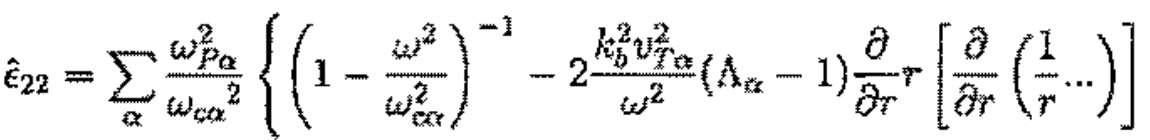

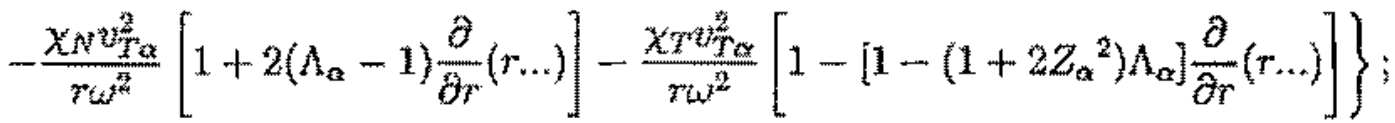

$$
\begin{aligned}
& \hat{\epsilon}_{2 s}=\sum_{\alpha \alpha} \frac{\omega_{P \alpha}^{2}}{\partial \omega_{c \alpha}}\left\{\Lambda_{\alpha} \frac{1}{k_{\|}} \frac{\partial}{\partial r}+\frac{\chi_{N}}{k_{l}} \Lambda_{\alpha}+\frac{\chi_{T}}{2 k_{n}}\left[1-\left(1-2 Z_{\alpha x}^{2}\right) \Lambda_{\alpha}\right]\right\}
\end{aligned}
$$

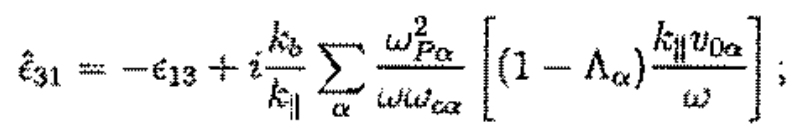

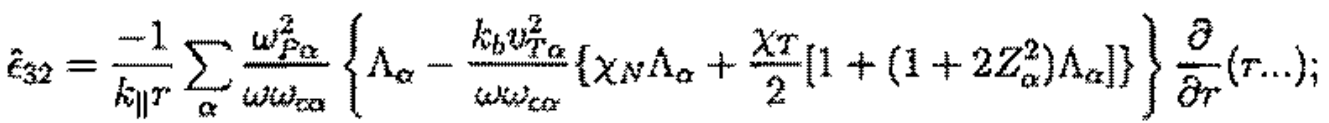




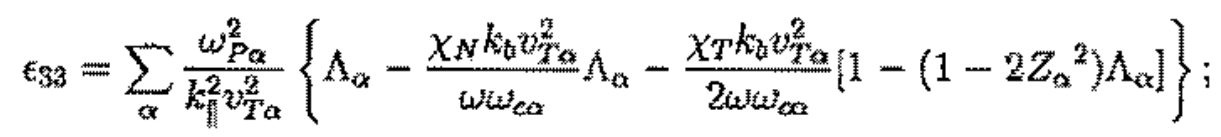

onde $w_{P_{\alpha}}^{2}=4 \pi N_{0 \alpha} e_{\alpha \alpha}^{2} / M_{\alpha} \dot{e}$ a frequiència de plasma,

$$
\Lambda_{\alpha}=1+i \sqrt{\pi} Z_{\alpha} W\left(Z_{\alpha}\right), \quad Z_{\alpha}=\frac{w-k_{\|} v_{0 \alpha}}{\sqrt{2} k_{\|} v_{T \alpha}}
$$

e

$$
W\left(z_{\alpha}\right)=\exp \left(-z_{\alpha \alpha}^{2}\right)\left[1+\frac{2 i}{\sqrt{\pi}} \int_{0}^{z_{\alpha}} \exp \left(t^{2}\right) d t\right]
$$

é a funçăo de dispersāo do plasma, e os parămetros de inomogeneidade radial $\chi_{1}, \chi_{N}, \chi_{T}$ são definidos como

$$
\chi_{N}=\frac{\partial}{\partial r} \ln N_{0}, \quad \chi_{T}=\frac{\partial}{\partial r} \ln T_{\alpha} .
$$




\section{B Tensor dielétrico}

Neste apêndice th listada a expressão completa do tensor dielétrico utilizado no código toroidal (2D). Alguns dos fragmentos e funçöas, como PDP $[x]$ (Plasma Dispersion Func-

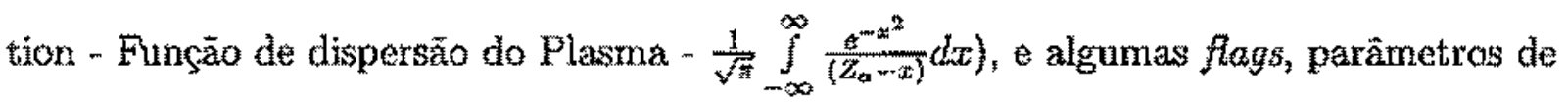
inclusão de efeitos opcionais são mantidos da forma em que são introduzidos no código. Estes sāo:
cove - Temo que sinaliza a existência ou näo de impurezas.
coupz-Termo que sinaliza a existência ou nāo de impurezas.
yzcoup - Termo de acoplamento de elementos r || e 1 . || do tenasor dielétrico.
scoup - Termo que sinaliza os efeitos de rato de Larmor fritu.

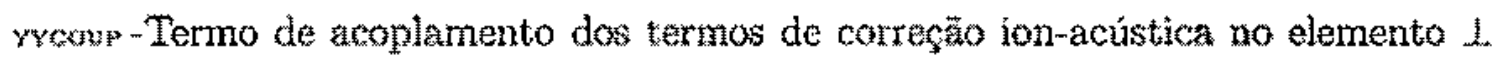

$\perp$ do tensor dielétrico.

Na maioria das componentes deste tensor, os termos de elétrons e lons estäo somados. Em alguns casos espectifoos, importantes principalmente para o cáloulo das forças ponderomotoras (Capitulo 4), os termos de íns e de elétrons estäo separados. 


$$
\begin{aligned}
& \chi_{r r}=\left(\frac{c}{\omega}\right)^{2}\left\{\left(\frac{\omega}{V_{A}(r)}\right)^{2} \cdot \alpha_{i}(r)-k_{\perp}^{2}(r) \cdot\left[\frac{3}{2} \rho_{i}^{2}(r)\left(\frac{\omega}{V_{A}(r)}\right)^{2} \alpha_{i}(r)+\right.\right. \\
& \frac{3 \rho_{\text {imp }}^{2}(r) \cdot \mathrm{F}}{4} \frac{\omega \cdot \omega_{p, \text { imp }}(r)}{c^{2}\left|k_{/ /}(r)\right| V_{\text {Timp }}(r)} \times\left(\operatorname{PDF}\left[\frac{\left(\omega-\Omega_{\text {imp }}\right)}{\left[k_{/ /}(r) \mid V_{\text {Timp }}(r)\right.}\right]+\operatorname{PDF}\left[\frac{\left(\omega+\Omega_{\text {imp }}\right)}{\left|k_{/ / /}(r)\right| V_{\text {Timp }}(r)}\right]\right) \cdot \text { coups }
\end{aligned}
$$

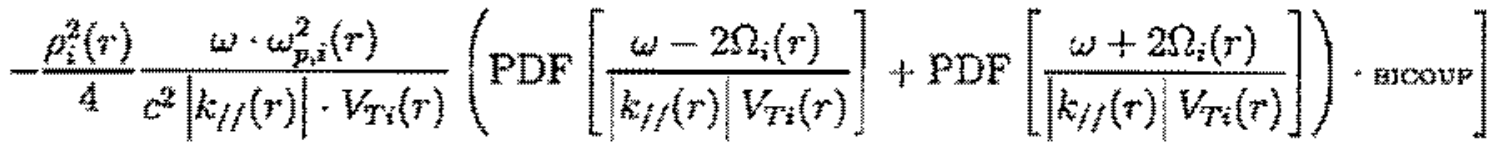

$$
\begin{aligned}
& +\frac{1}{2 a^{2}}\left[\left[\rho_{i}^{2}(r)\left(\frac{\omega}{V_{A}(r)}\right)^{2} \alpha_{\mathrm{i}}(r)\right]^{t}+\left[\rho_{\text {itrp }}^{2}(r) \cdot \mathrm{ZPI}(1 \mathrm{I}, \mathrm{J})\right]^{\prime}-\left[\rho_{i}^{2}(r) \cdot \mathrm{ZPI} 3\right]^{\prime}\right]\left[\frac{a}{r}+\frac{d}{d r}\right] \\
& +\frac{\text { RIW1SQ }}{a^{2}}\left[-\frac{a^{2}}{r^{2}}+\frac{a}{r} \frac{d}{d r}+\frac{d^{2}}{d r^{2}}\right]+
\end{aligned}
$$

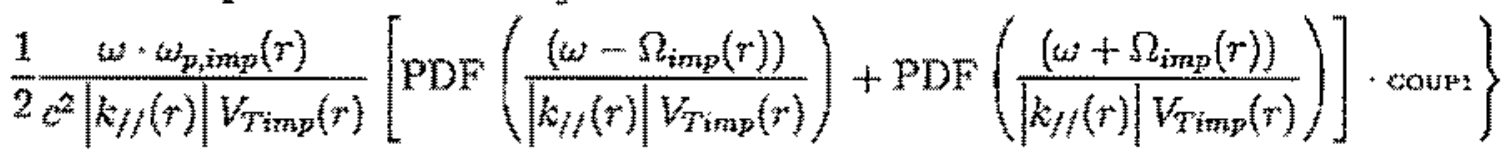

$$
\begin{aligned}
& \chi_{\perp \cdot r}=-i\left(\frac{c}{\omega}\right)^{2}\left\{\frac{\omega}{\Omega_{i}(r)}\left(\frac{\omega}{V_{A}(r)}\right)^{2} \alpha_{i}(r)-\frac{\mathrm{RIW} 1 S Q_{1}}{a} k_{\perp}(r)\left(\frac{a}{r}+\frac{d}{d r}\right)\right. \\
& +\frac{k_{\perp}(r)}{a}\left[\frac{3}{2}\left(\left[\rho_{i}^{2}(r)\left(\frac{\omega}{V_{A}(r)}\right)^{2} \alpha_{i}(r)\right]^{\prime}+\left[p_{i t u p}^{2}(r) \cdot \mathrm{ZPI1}(\mathrm{I}, \mathrm{J})\right]^{\prime}\right)-\left[\rho_{i}^{2}(r) \cdot \mathrm{ZPI} 3\right]^{\prime}\right]+ \\
& \mathrm{RIW} 3 \mathrm{SQ}_{\perp}(r)+\frac{\mathrm{RIW} 3 \mathrm{SQ}}{a} k_{\perp}(r) \frac{d}{d r}+\left[\frac{1}{2} \frac{\omega \cdot \omega_{p, i m p}(r) \cdot \mathrm{F}}{c^{2}\left|k_{\ell /(r)}\right| V_{T i m p}(r)} \times\right.
\end{aligned}
$$

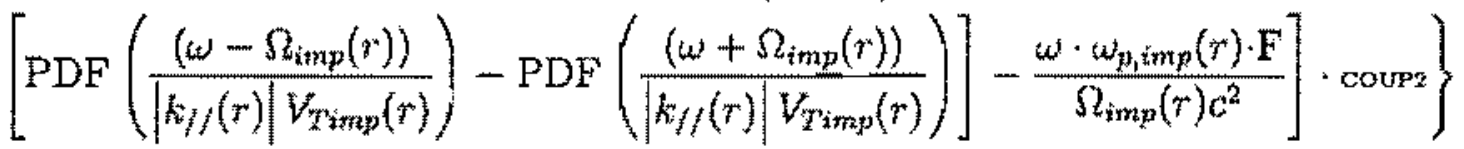

Para cálculos de forças ponderomotoras:

$$
\begin{aligned}
& \chi_{\perp r}^{i}=-i\left(\frac{c}{\omega}\right)^{2}\left\{\frac{\Omega_{i}(r)}{\omega}\left(\frac{\omega}{V_{A}(r)}\right)^{2} \alpha_{i}(r)-\frac{\mathrm{RIW}_{1} S Q_{k_{\perp}}(r)}{a}\left(\frac{a}{r}+\frac{d}{d r}\right)\right. \\
& +\frac{k_{\perp}(r)}{a}\left[\frac{3}{2}\left(\left[\rho_{i}^{2}(r)\left(\frac{\omega}{V_{A}(r)}\right)^{2} \alpha_{i}(r)\right]^{\prime}+\left[\rho_{\text {imp }}^{2}(r) \cdot \mathrm{ZPI}(\mathrm{I}, \mathrm{J})\right]^{\prime}\right)-\left[\rho_{i}^{2}(r) \cdot \mathrm{ZPI} 3\right]^{\prime}\right] \div
\end{aligned}
$$

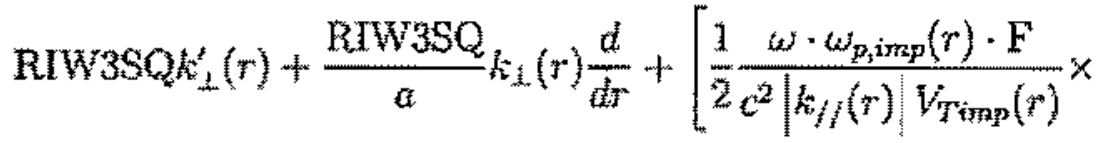

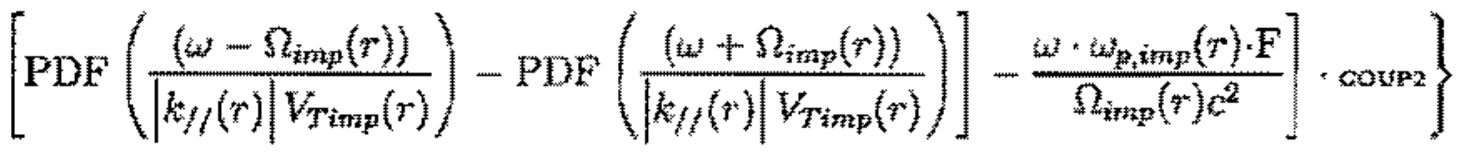




$$
\chi_{\perp r}^{e}=+i\left(\frac{c}{\omega}\right)^{2} \frac{\Omega_{e}(r)}{\omega}\left(\frac{\omega}{V_{A}(r)}\right)^{2} \alpha_{i}(r)
$$

$$
\begin{aligned}
& \chi_{r \perp}=i \cdot\left(\frac{c}{\omega}\right)^{2}\left\{\frac{\omega}{\Omega_{i}(r)}\left(\frac{\omega}{V_{A}(r)}\right)^{2} \alpha_{i}(r)-\frac{\mathrm{RIW} 3 S Q}{a} k_{\perp}(r)\left(\frac{a}{r}+\frac{d}{d r}\right)\right. \\
& +\frac{k_{\perp}(r)}{a} \frac{1}{2}\left[\left[\rho_{i}^{2}(r)\left(\frac{w}{V_{A}(r)}\right)^{2} \alpha_{i}(r)\right]^{\prime}+\left[\rho_{i m p}^{2}(r) \cdot \mathrm{ZPI}(1 \mathrm{I}, d)\right]^{t}-\left[\rho_{i}^{2}(r) \cdot \mathrm{ZPI} 3\right]^{\prime}\right] \\
& +\mathrm{RIW} 1 \mathrm{SQ} k_{\perp}^{t}(r)+\frac{\mathrm{R} W 1 \mathrm{SQ}}{a} k_{\perp}(r) \frac{d}{d r}+\left[\frac{1}{2} \frac{\omega \cdot \omega_{p, i m p}(r) \cdot \mathrm{F}}{c^{2}\left|k_{/}(r)\right| V_{T \text { imp }}(r)} \times\right.
\end{aligned}
$$

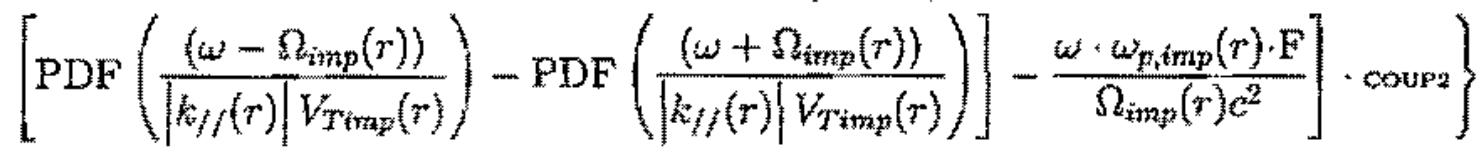

Para cálculos de forģns ponderomotoras:

$$
\begin{aligned}
& \chi_{r \perp}^{i}=+i\left(\frac{c}{\omega}\right)^{2}\left\{\frac{\Omega_{i}(r)}{\omega}\left(\frac{w}{V_{A}(r)}\right)^{2} \alpha_{i}(r)-\frac{\mathrm{RIW} 1 \mathrm{SQ}}{a} k_{\perp}(r)\left(\frac{a}{r}+\frac{d}{d r}\right)\right. \\
& +\frac{k_{\perp}(r)}{a}\left[\frac{3}{2}\left(\left[\rho_{i}^{2}(r)\left(\frac{\omega}{V_{A}(r)}\right)^{2} \alpha_{i}(r)\right]^{\prime}+\left[\rho_{i m p}^{z}(r) \cdot \mathrm{ZPH}(\mathrm{I}, J)\right]^{\prime}\right)-\left[\rho_{i}^{2}(r) \cdot \mathrm{ZPI}\right]^{\prime}\right]+ \\
& \mathrm{RIW} 3 S \mathrm{Q} k_{\mathrm{L}}^{i}(r)+\frac{\mathrm{RIW} 3 \mathrm{SQ}}{a} k_{\perp}(r) \frac{d}{d r}+\left[\frac{1}{2} \frac{\omega \cdot \omega_{p, i m p}(r) \cdot \mathrm{F}}{c^{2}\left|k_{/ /}(r)\right| V_{T \text { Tmp }}(r)} \times\right.
\end{aligned}
$$

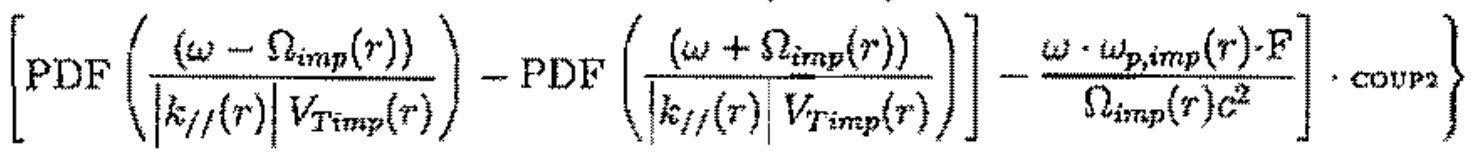

$$
\begin{aligned}
& \chi_{r+1}^{e}=-\hat{i}\left(\frac{b}{\omega}\right)^{2} \frac{\Omega_{E}(r)}{\omega}\left(\frac{\omega}{V_{A}(r)}\right)^{2} \alpha_{i}(r)
\end{aligned}
$$

$$
\begin{aligned}
& x_{\perp \perp}=\left(\frac{c}{\omega}\right)^{2}\left\{\left(\frac{\omega}{V_{A}(r)}\right)^{2} \cdot \alpha_{i}(r)-\operatorname{RrW1SQk} k_{\perp}^{2}(r)+\frac{\mathrm{DBBI} 2}{a^{2}} \cdot\left[\frac{\partial}{\partial r}+\frac{a}{r}\right]\right. \\
& \left.+\frac{\mathrm{RIW} 3 \mathrm{SQ}}{a^{2}}\left[-\left(\frac{a}{r}\right)^{2}+\frac{a}{r} \frac{\partial}{\partial r}+\frac{\partial^{2}}{\partial r^{2}}\right]+2 \mathrm{PII}\right\} \\
& \chi_{r \|}=-i k_{\perp}(r)\left(\frac{c}{V_{A}(r)}\right)^{2} \frac{\Omega_{i}(0) Z_{i}}{\left|k_{/ /}(r)\right| \omega}\left(1+\frac{\omega}{k_{/ /}(r) V_{T e}} \cdot \operatorname{PDF}\left[\frac{\omega}{k_{/ /}(r) V_{T e}}\right]\right) \cdot \text { yzcour }
\end{aligned}
$$




$$
\begin{aligned}
& \chi_{H}=-i k_{\perp}(r)\left(\frac{\epsilon}{V_{A}(r)}\right)^{2} \frac{\Omega_{i}(0) Z_{i}}{\left|k_{/ /}(r)\right| \omega}\left(1+\frac{\omega}{k_{/}(r) V_{T \mathrm{~T}}} \cdot \mathrm{PDF}\left[\frac{\omega}{k_{/ /}(r) V_{T e}}\right]\right) \cdot \mathrm{YZCOUP} \\
& \chi_{\text {내 }}=\frac{-1}{a}\left[\left(\frac{c}{V_{A}(r)}\right)^{2} \frac{\Omega_{i}(0) Z_{i}}{\left|k_{/ /}(r)\right| \omega}\left(1+\frac{\omega}{k_{/ l}(r) V_{T e}} \cdot \operatorname{PDF}\left[\frac{\omega}{k_{/ /}(r) V_{\mathrm{Te}}}\right]\right) \cdot \gamma_{\text {zooup }} \frac{\partial}{\partial r}\right. \\
& \left.+\left(\left(\frac{c}{V_{A}(r)}\right)^{2} \frac{\Omega_{i}(0) Z_{i}}{\left|k_{/ /}(r)\right| \omega}\left(1+\frac{\omega}{k_{/ /}(r) V_{r e}} \cdot \mathrm{PDF}\left[\frac{\omega}{k_{/ /}(r) V_{T e}}\right]\right) \cdot \text { Yzcour }\right)^{\prime}\right]
\end{aligned}
$$

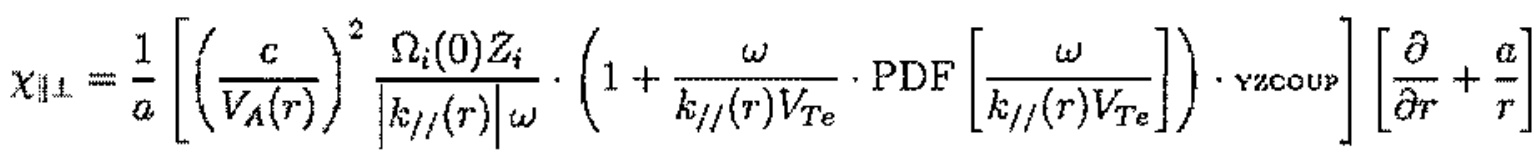

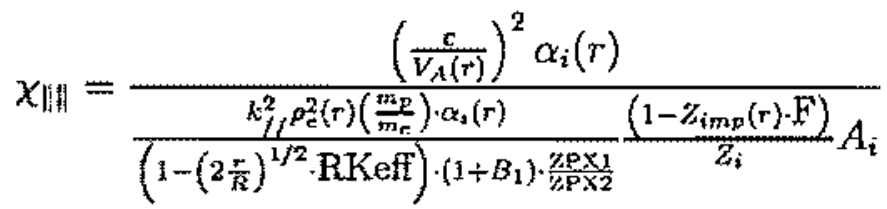

$$
\begin{aligned}
& \operatorname{RIW1SQ}(\mathrm{I}, \mathrm{J})=\frac{1}{2} p_{i}^{2}(r)\left(\frac{w}{V_{A}(r)}\right)^{2} \alpha_{i}(r)+
\end{aligned}
$$

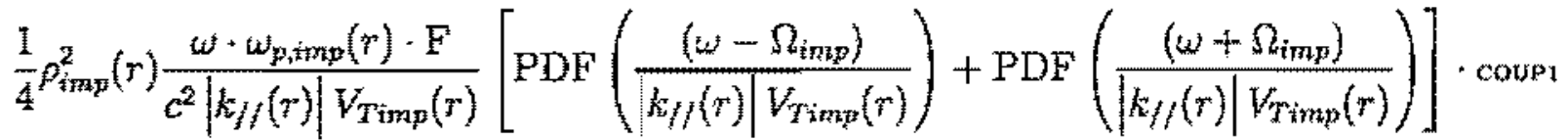

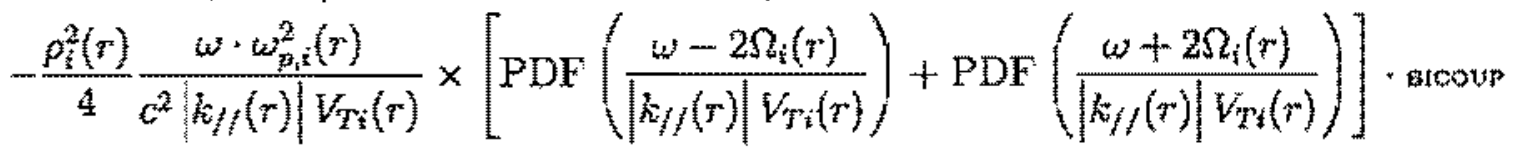

$$
\begin{aligned}
& \mathrm{RIW} 3 \mathrm{SQ}(1, J)=\frac{3}{2} p_{i}^{3}(r)\left(\frac{w}{V_{A}(r)}\right)^{2} \alpha_{i}(r)+
\end{aligned}
$$

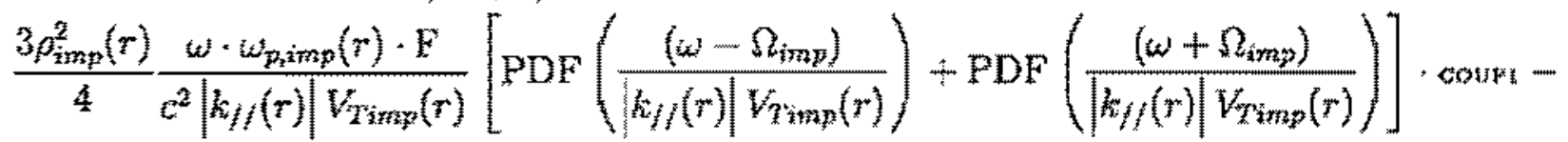

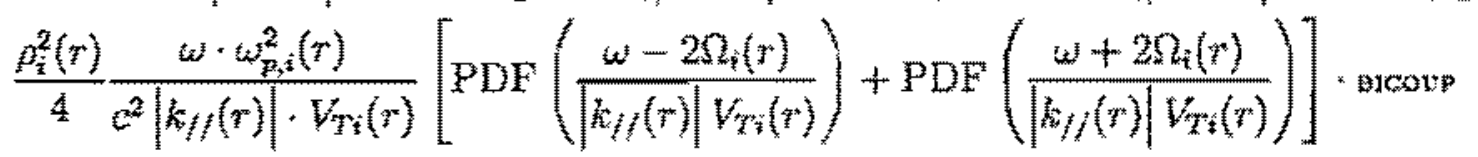

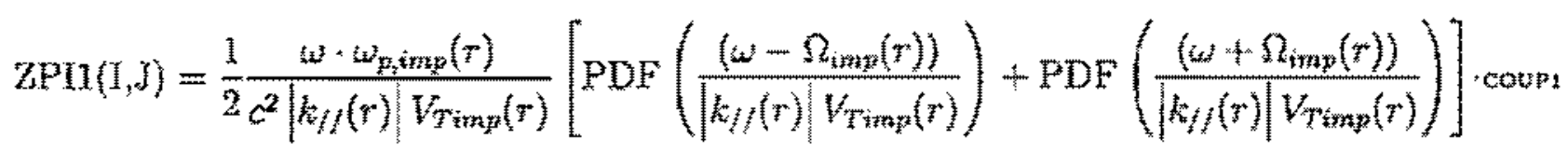

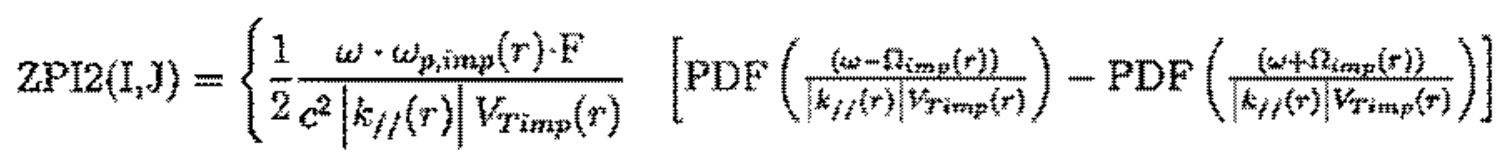

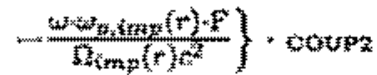




$$
\begin{gathered}
2 \mathrm{PI3}=\frac{1}{2} \frac{\omega \cdot \omega_{, i,}^{2}(r)}{c^{2}\left|k_{/ /}(r)\right| \cdot V_{r i}(r)}\left[\mathrm{PDF}\left(\frac{\omega-2 \Omega_{i}(r)}{\left|k_{/ /}(r)\right| \cdot V_{T i}(r)}\right)+\operatorname{PDF}\left(\frac{\omega+2 \Omega_{i}(r)}{\left|k_{/ /}(r)\right| \cdot V_{T i}(r)}\right)\right] \\
\alpha_{i}(r)=\frac{1}{1-\frac{\omega^{2}}{\Omega_{i}^{2}}}
\end{gathered}
$$

Componentes XY

$$
\begin{aligned}
& \chi_{r r X Y}=i \cdot\left(\frac{c}{\omega}\right)^{2} \frac{k_{\perp}(r)}{a}\left(\frac{a}{r} \mathrm{XXY}-\mathrm{XXY}^{3}\right)-i \cdot\left(\frac{c}{w}\right)^{2} k_{\perp}^{t}(r) \mathrm{XXY} \\
& X_{\perp \times X Y}=-\left(\frac{c}{\omega}\right)^{2}\left[\frac{\mathrm{XXY}}{a^{2}}\left(\frac{d^{2}}{d r^{2}}+\frac{a d}{r} \frac{d}{d r}-\frac{a^{2}}{r^{2}}\right)\right]+\frac{\mathrm{XXY}}{a^{2}}\left(\frac{d}{d r}+\frac{a}{r}\right)-k_{d}^{2}(r) \cdot \mathrm{XXY} \\
& X_{r \perp X Y}=\left(\frac{c}{\omega}\right)^{2}\left[\frac{\mathrm{XXY}}{a^{2}}\left(\frac{d^{2}}{d r^{2}}+\frac{a}{r} \frac{d}{d r}-\frac{a^{2}}{r^{2}}\right)\right]+\frac{\mathrm{XXY}}{a^{2}}\left(\frac{d}{d r}+\frac{a}{r}\right)-k_{\perp}^{2}(r) \cdot \mathrm{XXY} \\
& \chi_{\perp X Y}=i\left(\frac{c}{\omega}\right)^{2} k_{\perp}(r)\left\{\frac{\mathrm{XXY}}{r}-\frac{\mathrm{XXY}}{a}\right\}-i\left(\frac{c}{\omega}\right)^{2} k_{\perp}(r) \mathrm{XXY} \\
& T \chi_{* r}=\left(\frac{c}{V_{A}(r)}\right)^{2} \alpha_{i}(r) \\
& T \chi_{\perp \perp}=\left(\frac{c}{V_{A}(r)}\right)^{2} \alpha_{i}\{r) \\
& \mathrm{XXY}(\mathrm{I}, \mathrm{J})=\frac{3}{4} p_{i}^{2}(r)\left(\frac{\omega}{V_{A}(r)}\right)^{2} \alpha_{i}(r) \frac{\Omega_{i}(r)}{\omega} \cdot \mathrm{xrcoup}
\end{aligned}
$$

Termos da ressonância do segundo harmônico dos fons principais na corrente perturbada(COUP3 $=0)$

$$
\begin{aligned}
\chi_{r r 0} & =\left(\frac{c}{w}\right)^{2} \cdot 2 \mathrm{PI} 1 \\
\chi_{\perp r 0} & =-i\left(\frac{c}{\omega}\right)^{2} \cdot \mathrm{ZPI} 2 \\
\chi_{r \perp 0} & =i\left(\frac{c}{w}\right)^{2} \cdot \mathrm{ZPI} 2 \\
\chi_{\perp \perp 0} & =\left(\frac{c}{\omega}\right)^{2} \cdot \mathrm{ZPII} \cdot E_{\perp} \\
\chi_{r r 3} & =\left(\frac{c}{V_{A}(r)}\right)^{2} \alpha_{i}(r)
\end{aligned}
$$




$$
\begin{aligned}
& \chi_{1+3}=-i\left(\frac{c}{V_{A}(r)}\right)^{2} \alpha_{i}(r) \frac{\omega}{\Omega_{i}(r)} \\
& \chi_{+i 3}=i\left(\frac{c}{V_{A}(r)}\right)^{2} \alpha_{i}(r) \frac{\omega}{\Omega_{i}(r)} \\
& \chi_{d+13}=\left(\frac{c}{V_{A}(r)}\right)^{2} \cdot \alpha_{i}(r) \cdot E_{i}
\end{aligned}
$$

Correçöes para dissipação por tempo de trânsito com pi finito

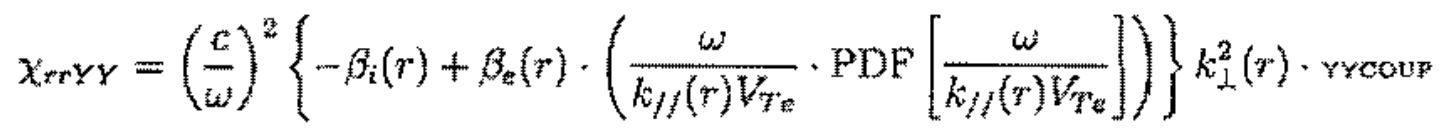

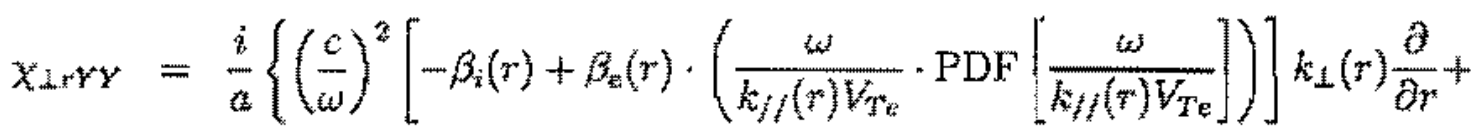

$$
\begin{aligned}
& {\left[\left\{\left(\frac{c}{\omega}\right)^{2}\left[-\beta_{i}(r)+\beta_{c}(r) \cdot\left(\frac{\omega}{k_{/}(r) V_{\text {Te }}} \cdot \operatorname{PDF}\left[\frac{\omega}{k_{/ /}(r) V_{r a}}\right]\right)\right]\right\}^{\prime} k_{\perp}(r)+\right.}
\end{aligned}
$$

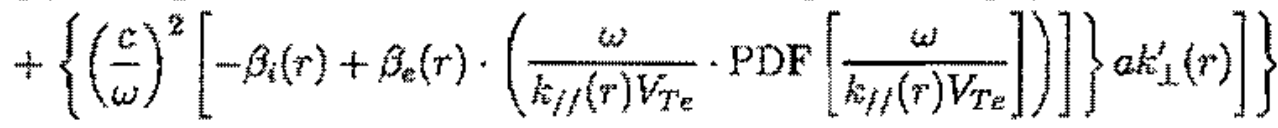

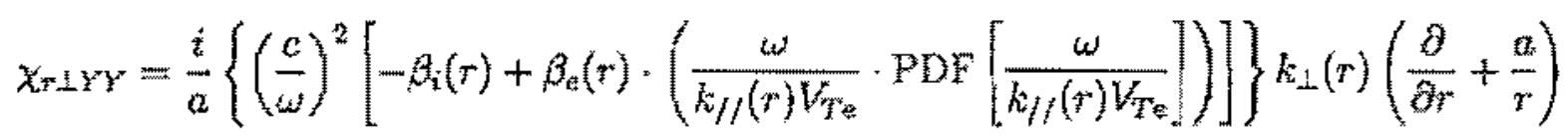

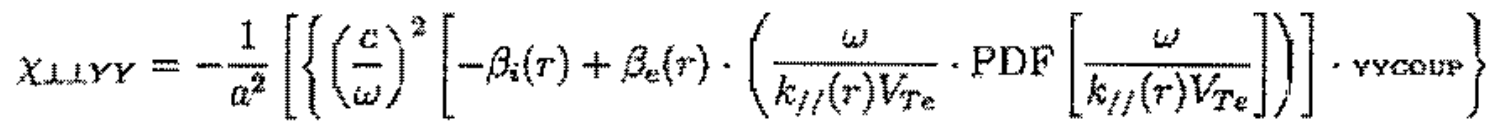

$$
\begin{aligned}
& \times\left[\frac{\partial^{2}}{\partial r^{2}}+\frac{a}{r} \frac{\partial}{\partial r} \frac{a}{r}-\frac{a^{2}}{r^{2}}\right]+
\end{aligned}
$$

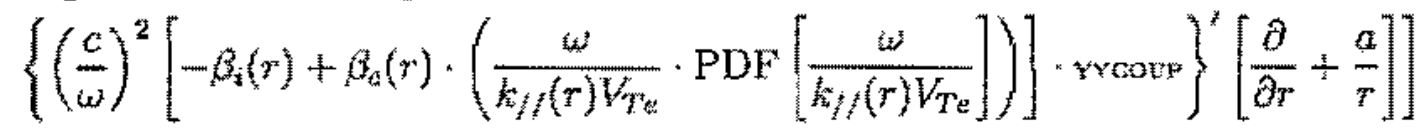




\section{Solução da Equação para o Campo Elétrico}

Neste apêndice é mostraxlo um exemplo de soluçäo das equaçöes de Maxwell para a obtençăo da expressão do campo eletrico com os valores do tensor dielétrico.

Partindo das Equaçós de Maxwell (9), obtéminse

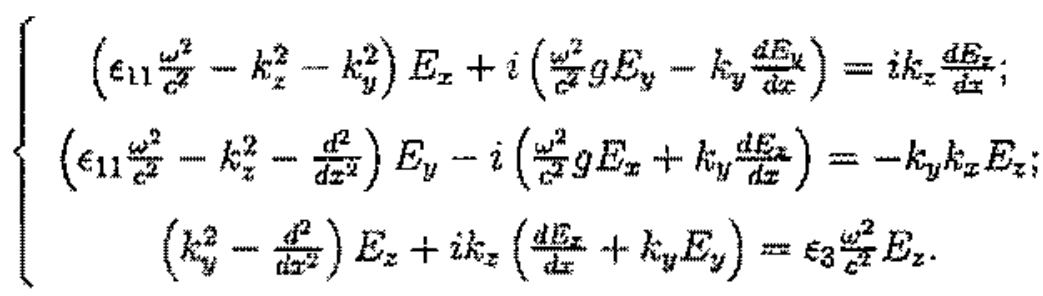

Da Eq. (118a),

$$
E_{x}=\frac{i k_{z} \frac{d E_{z}}{d x}-i\left(\frac{\omega^{2}}{\rho^{2} g}-k_{y} d z\right) E_{y}}{L},
$$

onde

$$
L=\left(\frac{w^{2}}{c^{2}} e_{1}-k_{y}^{2}-k_{z}^{2}\right)
$$

e, na notaçẫo usual do tensor dielétrico (Apendice 1),

$$
\varepsilon_{12} \equiv i g_{1} \text { e } \epsilon_{1} \equiv \varepsilon_{11}
$$

Fazendo-se, daqui por diante, para qualquer variável $A, \vec{A}=\frac{\psi^{2}}{c^{2}} A$,

$$
\begin{aligned}
& \left(\tilde{\epsilon}_{11}-k_{z}^{2}+\frac{d^{2}}{d x^{2}}\right) E_{y}+\left(\tilde{y}+k_{y} \frac{d}{d z}\right)\left(\frac{k_{z} \frac{d}{d x} E_{z}-\left(\tilde{g}+k_{y} \frac{d}{d x}\right) E_{y z}}{L}\right)=-k_{y z} k_{z} E_{z} \\
& \left(\frac{d^{2}}{d x^{2}}+\frac{\not{g} k_{y}}{L} \frac{d}{d x}\right) E_{y}-\quad k_{y} \frac{d}{d x}\left(\frac{g E_{y}}{L}\right)+\frac{d}{d x}\left(\frac{k_{y}^{2}}{L} \frac{d}{d x} E_{y}\right)+ \\
& \left(\widetilde{\epsilon}_{11}-k_{z}^{2}+\frac{\tilde{g}^{2}}{L}\right) E_{y}=-\left(k_{y} k_{z}+\frac{\ddot{g}+k_{y} \frac{d}{d x}}{L}\right) E_{z}
\end{aligned}
$$




$$
\begin{aligned}
& \frac{d}{d x}\left(\frac{\tilde{\varepsilon}_{11}-k_{z}^{2}}{\left(\tilde{\varepsilon}_{11}-k_{y}^{2}-k_{z}^{2}\right)} \frac{d}{d x} E_{y}\right)+ \\
& \left(\tilde{c}_{11}-k_{z}^{2}-\frac{\tilde{g}^{2}}{D}-k_{y} \frac{d}{d x}\left(\frac{\vec{g}}{L}\right)\right) E_{y}=-\left(k_{y} k_{z}+\frac{\ddot{g}+k_{y} \frac{d}{\partial z}}{L}\right) E_{z} .
\end{aligned}
$$

Supondo $E_{z}=\tilde{g}=0$ e $\tilde{E}_{11}=\tilde{\epsilon}_{0} x$,

$$
\frac{d}{d x}\left(\frac{\tilde{\epsilon}_{0} z-k_{z}^{2}}{\left(\tilde{\epsilon}_{11}-k_{y}^{2}-k_{z}^{2}\right)} \frac{d}{d x} E_{y}\right)+\left(\epsilon_{0} x-k_{z}^{2}\right) E_{y}=0
$$

$\operatorname{com} x_{0} \equiv \frac{k_{x}^{2}}{t_{0}}, x_{1}=\frac{k_{y}^{2}}{\epsilon_{0}}$ e $E_{y}=Y(x)$,

$$
\frac{d}{d x}\left(\frac{\left(x-x_{0}\right)}{\left(x-x_{0}-x_{1}\right)} \frac{d}{d x} Y(x)\right)+\tilde{\epsilon}_{0}\left(x-x_{0}\right) Y(x)=0 .
$$

Da série de Frobënsus

$$
Y(x)=\sum_{n=0}^{\infty} C_{n} x^{s+n}\left\{\begin{array}{c}
Y^{\prime}(x)=\sum_{n=0}^{\infty} C_{n}(s+n) x^{s+n-1} \\
Y^{\prime \prime}(x)=\sum_{n=0}^{\infty} C_{n}(s+n)(s+n-1), x^{s+n-3}
\end{array}\right.
$$

obtém-se a equaçäo que, aberta, fica como

$$
\begin{gathered}
\frac{\left(x-x_{0}\right)}{\left(x-x_{0}-x_{1}\right)} Y^{\prime \prime}(x)+\left(\frac{\left(x-x_{0}\right)}{\left(x-x_{0}-x_{1}\right)}\right)^{\prime} Y^{\prime \prime}(x)+\left(x-x_{0}\right) \tilde{\epsilon}_{0} Y(x)=0 \\
\frac{\left(x-x_{0}\right)}{\left(x-x_{0}-x_{1}\right)} Y^{\prime \prime}(x)+\left(\frac{\left(x-x_{0}-x_{1}\right)-\left(x-x_{0}\right)}{\left(x-x_{0}-x_{1}\right)^{2}}\right) Y^{\prime}(x)+\left(x-x_{0}\right) \tilde{\epsilon}_{0} Y(x)=0, \\
\left(x-x_{0}\right) Y^{\prime \prime}(x)-\left(\frac{x_{1}}{\left(x-x_{0}-x_{1}\right)^{2}}\right) Y^{\prime}(x) \div\left(x-x_{0}-x_{1}\right)\left(x-x_{0}\right) \tilde{\epsilon}_{0} Y(x)=0
\end{gathered}
$$




$$
\begin{aligned}
{\left[x^{2}-x\left(2 x_{0}+x_{1}\right)+x_{0}\left(x_{0}+x_{1}\right)\right] Y^{\prime \prime}(x)-x_{1} Y^{\prime}(x)+\left[x^{3}-x^{2}\left(3 x_{0}+2 x_{1}\right)\right.} & \\
& \left.+x_{(}\left(3 x_{0}^{2}+4 x_{0} x_{1}-x_{1}^{2}\right)-x_{0}\left(x_{0}+x_{1}\right)^{2}\right] \tilde{\epsilon}_{0} Y(x)=0(130)
\end{aligned}
$$

De outra mancira, fazendo $x-x_{0}=X^{0}$, obtém-se

$$
\left[X_{0}-x_{1}\right] X_{0} Y^{\prime \prime}\left(X_{0}\right)-x_{1} Y^{2}\left(X_{0}\right)+\left(X_{0}-x_{1}\right)^{2} X_{0} \tilde{E}_{0} Y\left(X_{0}\right)=0
$$

$$
\left[X_{0}-X_{0} x_{1}\right] Y^{m}\left(X_{0}\right)-x_{1} Y^{\prime}\left(X_{0}\right)+\left(X_{0}^{3}-2 X_{0}^{2} x_{1}+X_{0} x_{1}^{2}\right) \tilde{\epsilon}_{0} Y\left(X_{0}\right)=0
$$

com

$$
\begin{gathered}
Y\left(X_{0}\right)=\sum_{n=0}^{\infty} C_{n} x^{s+n} \\
Y^{\prime}\left(X_{0}\right)=\sum_{n=0}^{\infty} C_{n i}(s+n) x^{s+n-1} \\
Y^{n}\left(X_{0}\right)=\sum_{n=0}^{\infty} C_{n}(s+n)(s+n-1) x^{s+n-n} \\
\tilde{\epsilon}_{0} \sum_{n=0}^{\infty} C_{n} X_{0}^{s+n+3}-2 \tilde{\epsilon}_{0} x_{1} \sum_{n=0}^{\infty} C_{n} X_{0}^{s+n+2}+\tilde{\epsilon}_{0} x_{1}^{2} \sum_{n=0}^{\infty} C_{n} X_{0}^{s+n+1} \\
+\sum_{n=0}^{\infty} C_{n}(s+n)(s+n-1) X_{0}^{s+n}-x_{1} \sum_{n=0}^{\infty} C_{n}[(s+n)+(s+n)(s+n-1)] X_{0}^{s+n-1}=0
\end{gathered}
$$

A equaçấo indicial fornecida pela potèncina $X_{0}^{s+2 n-1}$, com $n=0$ é

$$
x_{1} C_{0}[s+s(s-1)]=0
$$

com a raiz dupla $s=0$, isso tanto para $x$ quanto $C_{0}$ diferentes de zero. Isolando as potências de $X_{0}$ e igualando a zero, obtém-se: 
parat $X_{0}^{3+4}$

$$
C_{1}=\frac{s(s-1)}{z_{1}(s+1)^{2}} C_{0}=0
$$

para $X_{0}^{s+n+1}$

$$
C_{2}=\frac{x_{1} \ddot{\varepsilon}_{0}}{4} C_{0}
$$

$\operatorname{para} X_{6}^{8+4+2}$

$$
c_{3}=-\frac{2 \epsilon_{0}}{3} c_{0}
$$

$\operatorname{para} X_{0}^{s+n+3}$

$$
C_{4}=-\frac{\tilde{\epsilon}_{0}}{16}\left(3-\frac{\tilde{t}_{1}^{3} \tilde{\epsilon}_{0}}{4}\right) C_{0}
$$

e assim por diante...

De posse de uma soluçäo linearmente independente, uma segunda é descoberta pela Commula $[66]$

$$
y_{2}(x)=C(x) y_{1}(x)
$$

onde

$$
C(X)=A \int_{a}^{x} \frac{e^{\left.-\int_{\xi_{1}}^{\xi} P(\eta)\right)(d)}}{\left[y_{1}(\xi)\right]^{2}} d \xi
$$

Para

$$
y^{*}(X)+P(X) y(X)+Q(X) y(X)=0
$$


onde $P(X)=-\frac{a_{1}}{X\left(x-x_{13}\right.}$ e $X=x-\frac{k_{y}^{2}}{x_{0}}$. Dal, utilizando apenas o primeiro termo da solução

$$
y_{1}=C_{0}+C_{2} \frac{x^{2}}{2} \ldots
$$

obtém-se

$$
\begin{aligned}
l(\xi)=-\int_{\varepsilon_{1}}^{\xi} \frac{x_{1}}{X\left(X-x_{1}\right)} d X=\int_{\xi}^{\xi}\left(\frac{1}{X}-\frac{1}{X-x_{1}}\right) \quad & \frac{x_{1}}{X\left(X-x_{1}\right)} d X= \\
& A^{\prime} \operatorname{Ln}(\xi)-B^{\prime} \operatorname{Ln}\left(\xi-x_{1}\right) .
\end{aligned}
$$

Fazendo $A^{\prime}=B^{\prime}=1$

$$
\begin{aligned}
& C(X)=\frac{A}{C_{0}^{2}} \int_{a}^{X} e^{-\operatorname{Ln}(\xi)+\operatorname{Ln}\left(\xi-x_{1}\right)} d \xi=A_{1} \int_{a}^{X} \frac{\left(\xi-x_{1}\right)}{\xi} d \xi= \\
& A\left[(X-a)-x_{1} \operatorname{Ln}(X)+x_{1} \operatorname{Ln}(a)\right]=X+x_{1} \operatorname{Ln}(X),
\end{aligned}
$$

e, assim, solucionada a equaça para a componente $y$ do campo elétrico,

$$
\begin{aligned}
& E_{y 1}=C_{0} \\
& E_{y 2}^{2}=C_{0}\left(x-\frac{k_{y}^{z}}{\widetilde{\epsilon}_{0}}+\frac{k_{y}^{2}}{\widetilde{\epsilon}_{0}} \ln \left(x-\frac{k_{y}^{2}}{\widetilde{\epsilon}_{0}}\right)\right) .
\end{aligned}
$$

A soluçäo para a componente $E_{z}$ é encontrada seguindo-se o mesmo caminho. Destas duas e mais a suposição de $E_{z}=0$, encontrà-se também o valor das componentes do campo magnótico. 


\section{Aplicando o Teorema de Poyting Complexo}

A expressão da energia eletromagnética é dada par

$$
\frac{\partial W}{\partial t}=\oint_{S} \vec{N} \cdot d \vec{S}-\frac{1}{2} \int_{V} \overrightarrow{J^{*}} \cdot \vec{E} d V
$$

onde o vetor de Poynting

$$
\vec{N}=\frac{c}{8 \pi} \vec{E} \times \overrightarrow{H^{*}}=\frac{c}{8 \pi \mu}\left(\vec{E} \times \overrightarrow{B^{*}}\right)
$$

e

$$
\frac{1 \partial \vec{B}}{\partial t}=-\vec{V} \times \vec{E}
$$

A soluçầo para o campo magnético é da forma

$$
\ddot{B}=\frac{-i c}{\omega}\left[-i k_{z} E_{y}, i k_{z} E_{x}, \cdots i k_{y} E_{x}\right]
$$

Como

$$
\begin{aligned}
& h_{y} E_{y} \cong-E_{x}\left(k_{z}^{2}+E_{y}^{2}\right), \\
& \vec{B}=\frac{c}{\omega}\left[-k_{z} E_{y}, i k_{z} E_{x y}-\frac{k_{z}^{2}}{k_{z}^{2}+k_{y}^{2}} E_{y}^{t}\right], \\
& \overrightarrow{B^{*}}=\frac{c}{\omega}\left[-k_{z} E_{y}^{*}, k_{*} E_{z}^{*}, \frac{k_{z}^{2}}{k_{z}^{2}+k_{y}^{2}} E_{y}^{* *}\right] .
\end{aligned}
$$

Assim,

$$
\vec{N}=\frac{c^{2}}{8 \pi \mu_{q} \omega^{*}}\left(i \frac{k_{z}^{2}}{k_{z}^{2}+k_{y}^{2}} E_{y} E_{y}^{\prime *},-i \frac{k_{z}^{2}}{k_{z}^{2}+k_{y}^{2}} E_{z} E_{y}^{\prime *}, k_{z} E_{x} E_{x}^{*}+k_{z} E_{y} E_{y}^{*}\right)
$$

o que fornece

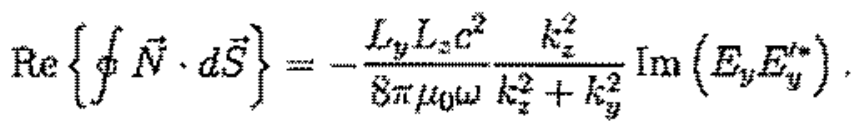


Das Equaçöes de Maxwell,

$$
\begin{aligned}
\tilde{y} & =\frac{c}{4 \pi}\left(i \frac{\omega}{c} \epsilon_{0} \vec{E}+\frac{1}{\mu_{0}} \vec{\nabla} \times \overrightarrow{B^{*}}\right) \\
& =\frac{c^{2}}{4 \pi \mu_{0} \omega}\left(i \frac{\omega^{2}}{c^{2}} \epsilon_{0} \mu_{0} \vec{E}+\left\{0,-i k_{z}^{2} E_{y}-i \frac{k_{z}^{2}}{k_{z}^{2}+k_{y}^{2}} E_{y}^{\prime \prime}, k_{z} E_{z}^{z}+i k_{y} k_{z} E_{y}\right\}\right),
\end{aligned}
$$

6

$$
\vec{J}^{*} \cdot \vec{E}^{4}=\frac{c^{2}}{4 \pi \mu_{0} \omega}\left(-\frac{\omega^{2}}{c^{2}} E_{0} \mu_{0}\left(E_{z}^{*} E_{x}+E_{y}^{*} E_{y i}\right)+i k_{z}^{2} E_{y}^{*} E_{y}-i \frac{k_{z}^{2}}{k_{z}^{2}+k_{y}^{2}} E_{y}^{* *} E_{y}\right) .
$$

a potêncida depositada no plasma, pela onda á

$$
\begin{aligned}
& \frac{1}{2} \int_{V} \overrightarrow{J *} \cdot \vec{E} d V= \\
& =\frac{L_{y} L_{z z} c^{2}}{8 \pi \mu_{0} \omega} \int\left(-\frac{w^{2}}{c^{2}} \epsilon_{0} \mu_{0} E_{z}^{*} \frac{k_{y}}{k_{z}^{2}+k_{y}^{2}} E_{y}^{i}-i\left(\frac{w^{2}}{c^{2}} \epsilon_{0} t_{0}-k_{z}^{2}\right) E_{y}^{*} E_{y}+\frac{E_{z}^{*}}{k_{y}} E_{y} k_{z}^{2}\right) d z_{z} \\
& =\frac{L_{y} L_{z} c^{2}}{8 \pi \mu_{0} \omega h_{y}}\left(-\frac{k_{y}^{2} k_{z}^{2}}{k_{z}^{2}+k_{y}^{2}} c_{0} \mu_{0} \int E_{x}^{*} E_{y}^{z} d x+k_{z}^{2} \int E_{x}^{*} E_{y} d x\right)
\end{aligned}
$$

Como

$$
\int E_{x}^{t} E_{y}^{\prime} d x=E_{y}^{*} E_{y}^{z}-\int E_{x}^{*} E_{y}^{\prime} d x=i \frac{k_{y}}{k_{z}^{2}+k_{y}^{2}} E_{y}^{* z} E_{y}-\int E_{x}^{*} E_{y}^{\prime} d x
$$

chega-se a

$$
\begin{aligned}
& \frac{1}{2} \int_{V} \cdot \vec{E} d V=
\end{aligned}
$$

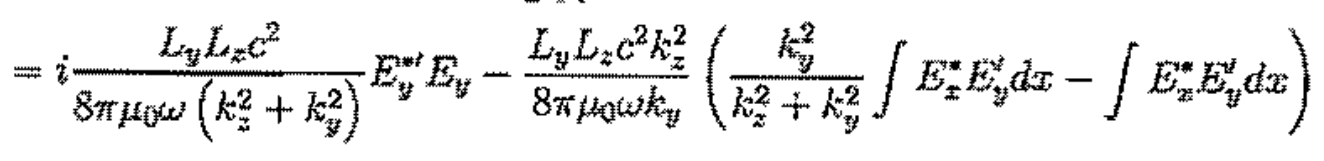

$$
\begin{aligned}
& \cong \frac{L_{i y} L_{z} c^{2}}{8 \pi \mu_{0} \omega^{2}\left(k_{z}^{2}+k_{y}^{2}\right)} E_{y}^{* \prime} E_{y}
\end{aligned}
$$

Usando a Eq. (54), chega-se a

$$
\operatorname{Re} \frac{1}{2} \int_{V} \breve{J}^{*} \cdot \vec{E} d v=\frac{L_{y} L_{z} c^{2}}{8 \pi f_{0} \omega\left(k_{z}^{2}+k_{y}^{2}\right)}\left|E_{y}\right|^{2} \pi \frac{r_{A}-r_{i}}{r_{A}}
$$




\section{E Construção do código toroidal}

\section{E.1 Resumo}

Neste apềndice, são mostrałos alguns dos cálculos realizados quando da elaboraçäo do código toroidal relatado no Capitulo 3.

Em primeiro lugar, é neceskátio mostrar o sistama de contanadas em que o código estẩ baseado:

$$
(r, \theta, z) \rightarrow(r ; \theta, b)
$$

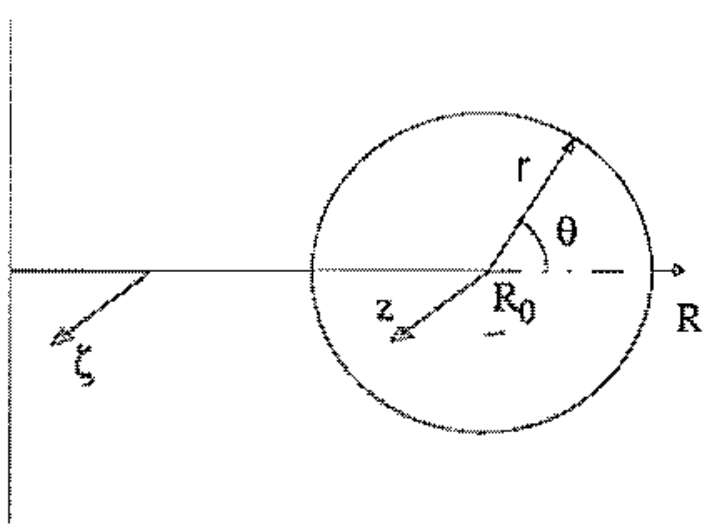

Figura E.1 - Esquema das coordenadas toroidais.

Estas coordanadas podern set transformatas seguindo

$$
\begin{aligned}
& Z=R_{0} S \\
& R=R_{0}+r \cdot \cos \theta
\end{aligned}
$$




$$
\begin{aligned}
& i \omega B_{r}=(\vec{\nabla} \times \vec{E})_{r}=-\frac{1}{R} \frac{\partial E_{\theta}}{\partial \zeta}+\frac{1}{r R} \frac{\partial}{\partial \theta}\left(R E_{z}\right) \\
& i \omega B_{\theta}=(\vec{\nabla} \times \vec{E})_{\theta}=\frac{1}{R} \frac{\partial E_{r}}{\partial \zeta}-\frac{1}{R} \frac{\partial}{\partial r}\left(R E_{z}\right) \\
& i \omega B_{\zeta}=(\vec{\nabla} \times \vec{E})_{z}=\frac{1}{r} \frac{\partial}{\partial r}\left(r E_{\theta}\right)-\frac{1}{r} \frac{\partial E_{r}}{\partial \theta} .
\end{aligned}
$$

As componentes de $\vec{\nabla} \times \vec{\nabla} \times \vec{E}$ podem ser escritas como

$$
\begin{aligned}
& (\vec{\nabla} \times \vec{\nabla} \times \vec{E})_{r}=-\frac{1}{R} \frac{\partial}{\partial \zeta}(\vec{\nabla} \times \vec{E})_{\theta}+\frac{1}{r R} \frac{\partial}{\partial \theta}\left[R(\vec{\nabla} \times \vec{E})_{z}\right] \\
& =-\left(\frac{1}{R^{2}} \frac{\partial^{2}}{\partial \zeta^{2}}+\frac{1}{r^{2} R} \frac{\partial}{\partial \theta} R \frac{\partial}{\partial \theta}\right) E_{r}+ \\
& \frac{1}{r^{2} R} \frac{\partial}{\partial \theta} R \frac{\partial}{\partial r} q E_{\theta}+\frac{1}{R^{2}} \frac{\partial}{\partial \zeta} \frac{\partial}{\partial r} R E_{*} \\
& (\vec{\nabla} \times \vec{\nabla} \times \vec{E})_{\theta}=-\frac{1}{R} \frac{\partial}{\partial \zeta}(\vec{\nabla} \times \vec{b})_{\tau}-\frac{1}{R} \frac{\partial}{\partial r}\left[R(\vec{\nabla} \times \vec{E})_{z}\right] \\
& =\frac{1}{R} \frac{\partial}{\partial r} \frac{R}{r} \frac{\partial}{\partial \theta} E_{r}-\left(\frac{1}{R^{2}} \frac{\partial^{2}}{\partial \zeta^{2}}+\frac{1}{R} \frac{\partial}{\partial r} \frac{R}{r} \frac{\partial}{\partial r} r\right) E_{\theta}+ \\
& \frac{1}{r R^{2}} \frac{\partial}{\partial \zeta} \frac{\partial}{\partial \theta} R E_{z} \text {; } \\
& (\vec{\nabla} \times \vec{\nabla} \times \vec{E})_{z}=\frac{1}{r} \frac{\partial}{\partial r} r(\vec{\nabla} \times \vec{E})_{\theta}-\frac{1}{r} \frac{\partial}{\partial \theta}(\vec{\nabla} \times \vec{E})_{r} \\
& =\frac{1}{r} \frac{\partial}{\partial r} r \frac{1}{R} \frac{\partial}{\partial \zeta} E_{r}+\frac{1}{r} \frac{\partial}{\partial \theta} \frac{1}{R} \frac{\partial}{\partial \zeta} E_{\theta} \\
& -\left(\frac{1}{r} \frac{\partial}{\partial r} r \frac{1}{R} \frac{\partial}{\partial r} R+\frac{1}{r} \frac{\partial}{\partial \theta} \frac{1}{r R} \frac{\partial}{\partial \theta} R\right) E_{*}
\end{aligned}
$$

Campo magnético de equilibrio

O campo magnético de equilibrio bidimensional é dado por

$$
\vec{B}_{0}=\frac{B_{0}\left(\hat{z}+\frac{r}{q R_{0}} \hat{\theta}\right)}{1+\frac{r}{R_{0}} \cos \theta}
$$

que satisfaz

$$
\ddot{\nabla} \cdot \overrightarrow{b_{0}}=0
$$


Note que

$$
B_{z}=\frac{B_{0} R_{0}}{R} \text { e } B_{0}=B_{0} \frac{r}{q R}=B_{z} \frac{r}{q R_{0}}
$$

e

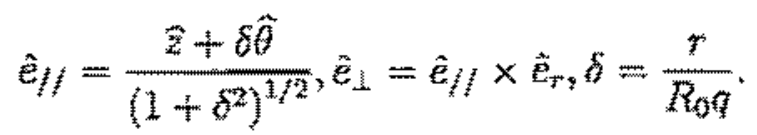

- Os vetores untátáos näo säo mudados pelos efettos toroidais.

- A magnitude de $\vec{B}_{0}$ é modificado pela curvatura toroidal

$$
\left|B_{0}\right|=\frac{B_{0}\left(1+\left(\frac{t}{q_{0}}\right)^{2}\right)^{1 / 2}}{1+\frac{5}{n_{0}} \cos \theta}
$$

As transformaçōes de coordenadas das componentes do campo eletrico säo dadas por

$$
\begin{aligned}
& E_{0}=\frac{E_{\perp}+\delta E_{i /}}{\left(1+\delta^{2}\right)^{1 / 2}}, \\
& E_{z}=\frac{E_{0}-\delta E_{z}}{\left(1+\delta^{2}\right)^{1 / 2}} \\
& E_{2}=\frac{E_{t}-\delta E_{1}}{\left(1+\delta^{2}\right)^{1 / 2}} \\
& E_{i y}=\frac{E_{0}-D E_{z}^{z}}{\left(1+\delta^{2}\right)^{1 / 2}} \\
& k_{\theta}=\frac{k_{1}+\delta k_{h /}}{\left(1+\delta^{2}\right)^{1 / 2}} \\
& k_{k}=\frac{k_{g}-\delta k_{z}}{\left(1+\delta^{2}\right)^{1 / 2}} \\
& k_{z}=\frac{k_{/}-\delta k_{1}}{\left(1+\delta^{2}\right)^{1 / 2}} \text {, } \\
& k_{/ 1}=\frac{k_{0}-\delta h_{z}}{\left(1+\delta^{2}\right)^{1 / 2}} \text {, }
\end{aligned}
$$

onde

$$
\delta=\frac{r}{a R_{i}}
$$

Fazendo a evolução dos termos da Eq. de onda,

$$
\begin{aligned}
& (\vec{\nabla} \times \vec{\nabla} \times \vec{E})_{r}=-\left(\frac{1}{R^{2}} \frac{\partial^{2}}{\partial \zeta^{2}}+\frac{1}{r^{2} R} \frac{\partial}{\partial \theta} R \frac{\partial}{\partial \theta}\right) E_{\mathrm{r}}+ \\
& \left(\frac{1}{r^{2} R} \frac{\partial}{\partial \theta} R \frac{\partial}{\partial r} \frac{r}{\left(1+\delta^{2}\right)^{1 / 2}}-\frac{1}{R^{2}} \frac{\partial \zeta}{\partial(\partial r} \frac{\partial}{\left(1+\delta^{2}\right)^{1 / 2}}\right) E_{1}+ \\
& \left(\frac{1}{r^{2} R} \frac{\partial}{\partial \theta} R \frac{\partial}{\partial r} \frac{r \delta}{\left(1+\delta^{2}\right)^{1 / 2}}+\frac{1}{R^{2}} \frac{\partial}{\partial \zeta} \frac{\partial}{\partial r\left(1+\delta^{2}\right)^{1 / 2}}\right) E_{1 /}
\end{aligned}
$$




$$
\begin{aligned}
& (\vec{\nabla} \times \vec{\nabla} \times \vec{E})_{\perp}=\left[\frac{1}{R\left(1+\delta^{2}\right)^{1 / 2}} \frac{\partial}{\partial r} \frac{R}{r \theta}-\frac{\delta R}{\left(1+\delta^{2}\right)^{1 / 2}} \frac{\partial}{\partial r} \frac{r}{R} \frac{\partial}{\partial \zeta}\right] E_{r} \\
& -\left[\frac{1}{R\left(1+\delta^{2}\right)^{1 / 2}}\left(\frac{\partial}{\partial \zeta}+\frac{\delta R}{r} \frac{\partial}{\partial \theta}\right) \frac{1}{R}\left(\frac{\partial}{\partial \zeta}+\frac{1}{r} \frac{\partial}{\partial \theta} R \delta\right) \frac{1}{\left(1+\delta^{2}\right)^{1 / 2}}\right. \\
& \left.+\frac{1}{R\left(1+\delta^{2}\right)^{1 / 2}} \frac{\partial r}{\partial r} \frac{R}{\partial r} \frac{r}{\left(1+\delta^{2}\right)^{1 / 2}}+\frac{\delta}{r\left(1+\delta^{2}\right)^{1 / 2}} \frac{\partial r}{\partial r} \frac{\partial}{\partial r} \frac{\delta R}{\left(1+\delta^{2}\right)^{1 / 2}}\right] E_{\perp} \\
& -\left[\frac{1}{R\left(1+\delta^{2}\right)^{1 / 2}}\left(\frac{\partial}{\partial \zeta}+\frac{\delta R}{r} \frac{\partial}{\partial \theta}\right) \frac{1}{R}\left(\frac{\delta}{r} \frac{\partial}{\partial \zeta}-\frac{1}{r} \frac{\partial}{\partial \theta}\right) \frac{R}{\left(1+\delta^{2}\right)^{1 / 2}}\right. \\
& \left.+\frac{1}{R\left(1+\delta^{2}\right)^{1 / 2}} \frac{\partial r}{\partial r} \frac{R}{\partial r} \frac{\delta r}{\left(1+\delta^{2}\right)^{1 / 2}}-\frac{\delta}{r\left(1+\delta^{2}\right)^{1 / 2}} \frac{\partial}{\partial r} \frac{\partial}{R} \frac{R}{\left(1+\delta^{2}\right)^{1 / 2}}\right] E_{l /},(1)
\end{aligned}
$$

\section{E.1.1 Equaçăo da onda}

O programa resolve a eqุiaçăo

$$
\frac{\vec{w}^{2}}{c^{2}} \overleftrightarrow{\vec{X}} \cdot \vec{E}-\vec{\nabla} \times \vec{\nabla} \times \vec{E}=0
$$

onde $\overleftrightarrow{\chi}$ é o tensor dielétrico cinético $\left.{ }^{20}\right]$.

Ao invés de $(\vec{\nabla} \times \vec{\nabla} \times \vec{E})_{\eta}$, é usado

$$
\vec{\nabla} \cdot \vec{J}=\frac{1}{r R} \frac{\partial}{\partial r} r R J_{x}+\frac{1}{r R} \frac{\partial}{\partial \theta} R J_{\theta}+\frac{1}{R} \frac{\partial}{\partial \zeta} J_{z}=0
$$

onde a toroidicidade é desprezada, e

$$
R=R_{0}
$$

entä̊, chega-se à expressüo

$$
\frac{1}{r} \frac{\partial}{\partial r} r J_{r}+\frac{1}{r} \frac{\partial}{\partial \theta} J_{\theta}+\frac{1}{R_{0}} \frac{\partial}{\partial \zeta} J_{z}=0
$$

our, com

$$
\begin{aligned}
& \hat{e}_{/ /}=\frac{\hat{z}+\delta \hat{\theta}}{\left(1+\delta^{2}\right)^{1 / 2}}, \quad \hat{e}_{i}=\hat{e}_{\eta /} \times \hat{e}_{r} \text { e } \delta=\frac{r}{R q}, \\
& i\left(k_{/ /} J_{H}+k_{\perp} J_{L}\right)+\frac{1}{r} \frac{\partial}{\partial r} r J_{r}=0 .
\end{aligned}
$$


É entāo usada a equação

$$
\ddot{J}=\frac{1}{i t_{0} \omega} \frac{\omega^{2}}{c^{2}} \overleftrightarrow{x} \cdot \vec{E}
$$

para expressar $E_{/ /}$err termos de $E_{r}$ e $E_{1}$.

A toroldicidade é levada em conta por meio das seguintes aproximaçōes:

$$
\begin{aligned}
\frac{1}{R} & =\frac{1}{R_{0}}\left(1-\frac{r}{R_{0}} \cos \theta\right), \\
\frac{1}{R^{2}} & =\frac{1}{R_{0}^{2}}\left(1-2 \frac{r}{R_{0}} \cos \theta\right) .
\end{aligned}
$$

\section{E.1.2 Transformada de Fourier}

Depois de ter expandido todas as componentes da equaçäo da onda, separando-as nas partes cilindricas e nas partes de correçào toroidal, os coeficientes da equaçäo säo separados nos operadores $\widehat{L}_{0}, \widehat{L}_{\mathcal{S}}$ e $\widehat{L}_{A}$ :

$$
\begin{aligned}
\vec{L} \vec{E}=\hat{L}_{0} \vec{E} \text { (parte cilíndrica) } & \\
& +\left(2 \frac{r}{R_{0}} \cos \theta \hat{L}_{S}+2 \frac{r}{R_{0}} \sin \theta \hat{L}_{A}\right) \vec{E}=0
\end{aligned}
$$

e, com

$$
\vec{E}=\sum_{m} \vec{E}_{m}(r) \exp i(m \theta-l \zeta)
$$

E efetuada uma tratnsformação de Fourier jara us primeiros modos poloidais vizinhos:

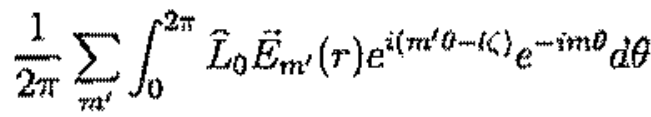

$$
\begin{aligned}
& +\frac{1}{2 \pi} \frac{r}{R_{0}} \sum_{m^{\prime}} \int_{0}^{2 \pi}\left(\bar{L}_{S}+\overleftrightarrow{L}_{A}\right) \vec{E}_{m^{\prime}}(r) e^{i\left(m^{\prime} \theta-l \zeta\right)} e^{-i(m-1) \theta} d \theta \\
& +\frac{1}{2 \pi} \frac{r}{R_{0}} \sum_{m m^{i}} \int_{0}^{i \pi}\left(\hat{L}_{S}-\hat{L}_{A}\right) \vec{E}_{m}(r) e^{i(m+\theta-t c)} e^{-i(m+1) t} d \theta \\
& =0 \text {, }
\end{aligned}
$$


o gue leva a

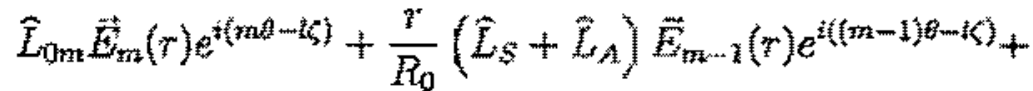

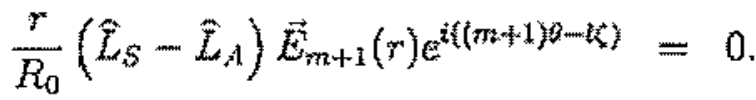

Para $\hat{e}=\frac{r}{r_{3}}$ näo nulo, é considerado o acoplamento de 3 modos poloidais e urm sistema de sexta ordem e resolvido:

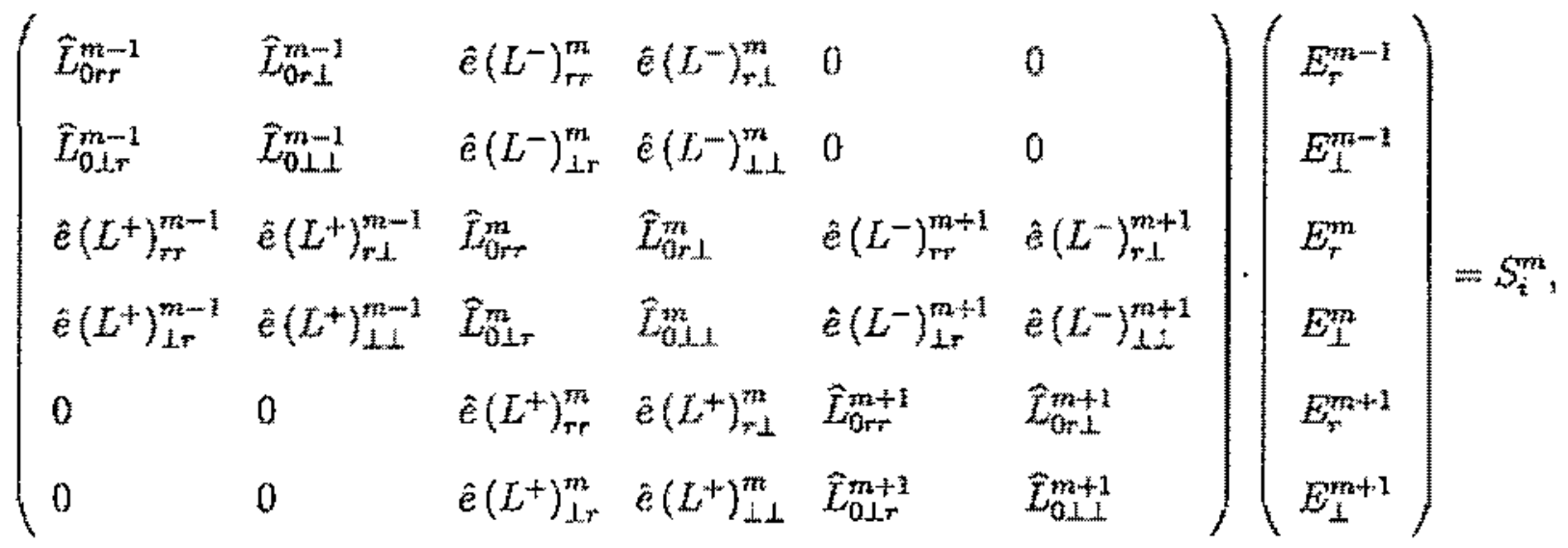

onde $L^{+}=\bar{L}_{S}+\hat{L}_{A} ; L^{-}=\widehat{L}_{S}-\vec{L}_{A}$.

Este sistema esolvido com uma spline-cúbica é obtido como resposta o perfil das componentes radial e perpendicular do campo elétrico. A componente paralela é então calculada pela hipótese de incompressibilidade expressa ná Eq.(173).

\section{E.1.3 Conservação de Energia}

Do teorema de Poynting:

$$
\operatorname{Re} \int \ddot{\nabla} \cdot \vec{S} d v=\operatorname{Re} \int \ddot{E} \cdot \ddot{v} d v
$$

ou

$$
\operatorname{Re} \int S_{r} d A=R e \int E \cdot H^{*} d v
$$

ondes

$$
\vec{S}=\frac{\vec{E} \times \vec{B}^{*}}{\mu_{0}} ; d v=r R d r d \theta d \zeta ; d A=r R d \theta d \zeta
$$


Tal condição é expressa no código na forma

$$
\begin{aligned}
& \frac{2 \pi r R_{0}}{\mu_{0}} \operatorname{Re}\left[\left(\vec{E}_{m} \times \vec{B}_{m}^{*}\right)_{r}+\frac{\tilde{e}}{2}\left(\vec{E}_{m} \times \vec{B}_{m+1}^{*}\right)_{r}+\frac{\hat{e}}{2}\left(\vec{E}_{m} \times \vec{B}_{m-1}^{*}\right),\right. \\
& +\frac{\hat{E}}{2}\left(\vec{E}_{m+1} \times \vec{B}_{m+1}^{*}\right)_{*}+\frac{\hat{e}}{2}\left(\vec{E}_{m+1} \times \vec{B}_{m}^{*}\right)_{r} \\
& \left.+\frac{\hat{e}}{2}\left(\vec{B}_{m-1} \times \vec{B}_{m-1}^{*}\right)+\frac{\hat{e}}{2}\left(\vec{E}_{m-1} \times \vec{B}_{m a}^{*}\right)_{r}\right]
\end{aligned}
$$

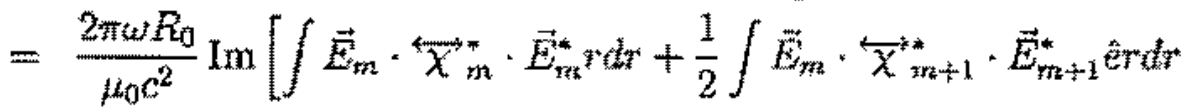

$$
\begin{aligned}
& +\frac{1}{2} \int \vec{E}_{m i} \cdot \overleftrightarrow{X}_{m+1}^{*} \cdot \vec{E}_{r n-1}^{*} \hat{e} r d r \\
& +\int \vec{E}_{m+1} \cdot \overleftrightarrow{X}_{m+1}^{*} \cdot \ddot{E}_{m+1}^{*} \hat{E r} d t+\frac{1}{2} \int \vec{E}_{m+1} \cdot \overleftrightarrow{X}_{m}^{* *} \cdot \vec{E}_{m}^{*} \hat{e r} d r \\
& \left.+\int \vec{E}_{m-1}^{*} \cdot \overleftrightarrow{X}_{m-1}^{*} \cdot \vec{E}_{m-1}^{*} \hat{e} r d r+\frac{1}{2} \int \vec{E}_{m-1}^{*} \cdot \overleftrightarrow{\chi}_{m}^{*} \cdot \vec{E}_{m}^{*} e^{*} r d r\right] \text {. }
\end{aligned}
$$

No cồdigo, algumas componentes na expressŏo da deposição de energia (chamado Fluxo Cinético) tẫo movidas para a parte fluxo de Poynting para assegurar que a deposiçẫo de energia é positiva. 


\section{E.2 Cálculos da Equaçāo da onda}

Da Equação da onda de Maxwell.

$$
\frac{\omega^{2}}{c^{2}} \vec{X} \cdot \vec{E}-\vec{\nabla} \times \vec{\nabla} \times \vec{E}=0
$$

onde

$$
\begin{aligned}
& E_{\theta}=\frac{E_{1}+\delta E_{i}}{\left(1+\delta^{2}\right)^{1 / 2}}, \\
& E_{1}=\frac{E_{0}-\delta E_{z}}{\left(1+\delta^{2}\right)^{1 / 2}} \\
& E_{z}=\frac{E_{i}-\delta E_{1}}{\left(1+\delta^{2}\right)^{1 / 2}} \\
& E_{n}=\frac{E_{z}+\delta E_{\theta}}{\left(1+\delta^{2}\right)^{1 / 2}}
\end{aligned}
$$

Separando as componentes do lado direito,

$$
\begin{aligned}
& (\vec{\nabla} \times \vec{\nabla} \times \vec{E})_{r}=-\left[\frac{1}{R^{2}} \frac{\partial^{2}}{\partial \zeta^{2}}+\frac{1}{r^{2} R} \frac{\partial}{\partial \theta} R \frac{\partial}{\partial \theta}\right] E_{r}+ \\
& \frac{1}{r^{2} R} \frac{\partial}{\partial \theta} R \frac{\partial}{\partial r}\left(r \frac{E_{1}+\delta E_{\|}}{\sqrt{1+\delta^{2}}}\right)+\frac{1}{R^{2}} \frac{\partial}{\partial \zeta} \frac{\partial}{\partial r}\left(R \frac{E_{\|}-\delta E_{\perp}}{\sqrt{1+\delta^{2}}}\right) \\
& (\vec{\nabla} \times \vec{\nabla} \times \vec{E})_{+}=-\left[\frac{1}{R^{2}} \frac{\partial^{2}}{\partial r^{2}}+\frac{1}{r^{2} R} \frac{\partial}{\partial \theta} R \frac{\partial}{\partial \theta}\right] E_{r}+ \\
& {\left[\frac{1}{r^{2} R} \frac{\partial}{\partial \theta} R \frac{\partial}{\partial r^{2}} \frac{r}{\sqrt{1+\delta^{2}}}-\frac{1}{R^{2}} \frac{\partial}{\partial \delta} \frac{\partial}{\partial r} \frac{\delta R}{\sqrt{1+\delta^{2}}}\right] E_{1}+} \\
& {\left[\frac{1}{r^{2} R} \frac{\partial}{\partial \theta} R \frac{\partial}{\partial r} \frac{\delta r}{\sqrt{1+\delta^{2}}}+\frac{1}{R^{2}} \frac{\partial}{\partial \zeta} \frac{\partial}{\partial r} \frac{R}{\sqrt{1+\delta^{2}}}\right] E_{\|}} \\
& (\vec{\nabla} \times \vec{\nabla} \times \vec{E})_{1}=\frac{(\vec{\nabla} \times \vec{\nabla} \times \vec{E})_{\partial}-\delta(\vec{\nabla} \times \vec{\nabla} \times \vec{E})_{2}}{\sqrt{1+\delta^{2}}}= \\
& \frac{1}{\sqrt{1+\delta^{2}}}\left[\frac{1}{R} \frac{\partial}{\partial r} \frac{R}{r} \frac{\partial}{\partial \theta} E_{r}-\left(\frac{1}{R^{2}} \frac{\partial^{2}}{\partial \zeta^{2}}+\frac{1}{R} \frac{\partial}{\partial r} \frac{R}{r} \frac{\partial}{\partial r}\right) E_{\theta}+\frac{1}{r R^{2}} \frac{\partial}{\partial \zeta} \frac{\partial}{\partial \theta} R E_{z}\right] \\
& -\frac{\delta}{\sqrt{1+\delta^{2}}}\left[\frac{1}{r} \frac{\partial}{\partial r} \frac{r}{R} \frac{\partial}{\partial \zeta} E_{r}+\frac{1}{\partial} \frac{\partial}{\partial \partial} \frac{1}{R} \frac{\partial}{\partial \zeta} E_{\theta}-\left(\frac{1}{r} \frac{\partial}{\partial r} \frac{\partial}{R} \cdot \frac{\partial}{\partial r}+\frac{1}{r} \frac{\partial}{\partial \theta} \frac{1}{r R} \frac{\partial}{\partial \theta} R\right) E_{z}\right]
\end{aligned}
$$




$$
\begin{aligned}
& (\vec{\nabla} \times \vec{\nabla} \times \vec{E})_{1}=\left[\frac{1}{R \sqrt{1+\delta^{2}}} \frac{\partial R}{\partial r} \frac{R}{\partial \theta}-\frac{\delta}{r \sqrt{1+\delta^{2}} \frac{\partial}{\partial r}} \frac{r}{R} \frac{\partial}{\partial \zeta}\right] E_{r}- \\
& {\left[\frac{1}{R \sqrt{1+\delta^{2}}}\left(\frac{\partial}{\partial \zeta}+\frac{\delta R}{r} \frac{\partial}{\partial \theta}\right) \frac{1}{R}\left(\frac{\partial}{\partial \zeta}+\frac{1}{r} \frac{\partial}{\partial \theta} \delta R\right) \frac{1}{\sqrt{1+\delta^{2}}}+\right.} \\
& \left.\frac{1}{R \sqrt{1+\delta^{2}}} \frac{\partial}{\partial r} \frac{R}{\partial r} \frac{r}{\sqrt{1+\delta^{2}}}+\frac{\partial}{r \sqrt{1+\delta^{2}}} \frac{\partial r}{\partial r} \frac{\partial}{\partial r} \frac{\delta R}{\sqrt{1+\delta^{2}}}\right] E_{\perp}+ \\
& {\left[\frac{1}{R \sqrt{1+\delta^{2}}}\left(\frac{\partial}{\partial \zeta}+\frac{\partial R \partial}{\partial \theta}\right) \frac{1}{R}\left(\frac{\delta}{R} \frac{\partial}{\partial \zeta}-\frac{1}{\partial \partial \theta}\right) \frac{R}{\sqrt{1+\delta^{2}}}+\right.} \\
& \left.\frac{1}{R \sqrt{1+\delta^{2}}} \frac{\partial}{\partial r} \frac{R}{r} \frac{\partial}{\partial r} \frac{r}{\sqrt{1+\delta^{2}}}-\frac{\delta}{r \sqrt{1+\delta^{2}}} \frac{\partial}{\partial r} \frac{r}{R} \frac{\partial}{\partial r} \frac{R}{\sqrt{1+\delta^{2}}}\right] E_{\|}
\end{aligned}
$$

A partir de agora seräo fẹitas as transformaçöes para correção toroidal na Eq.[179] e usando $\delta=\frac{r}{R q}=\frac{r}{R_{0 q}}$.

\section{E.2.1 Componente radial}

$$
\begin{aligned}
& (\vec{\nabla} \times \vec{\nabla} \times \vec{E})_{r}=-\left[\frac{1}{R^{2}} \frac{\partial^{2}}{\partial \zeta^{2}}+\frac{1}{r^{2} R} \frac{\partial}{\partial \theta} R \frac{\partial}{\partial \theta}\right] E_{\tau}+ \\
& {\left[\frac{1}{r^{2} R} \frac{\partial}{\partial \theta} R \frac{\partial}{\partial r} \frac{r}{\sqrt{1+\delta^{2}}}-\frac{1}{R^{2}} \frac{\partial}{\partial \zeta} \frac{\partial}{\partial r} \frac{\delta R}{\sqrt{1+\delta^{2}}}\right] E_{\perp}+} \\
& {\left[\frac{1}{r^{2} R} \frac{\partial}{\partial \theta} R \frac{\partial}{\partial r} \frac{\delta r}{\sqrt{1+\delta^{2}}}+\frac{1}{R^{2}} \frac{\partial}{\partial \zeta} \frac{\partial}{\partial r} \frac{R}{\sqrt{1+\delta^{2}}}\right] E_{t}} \\
& (\vec{\nabla} \times \vec{\nabla} \times \vec{E})_{r}= \\
& -\left[\frac{\left(1-2 \frac{r}{R_{0}} \cos \theta\right)}{R_{0}^{2}} \frac{\partial^{2}}{\partial \zeta^{2}}+\frac{\left(1-\frac{r}{R_{0}} \cos \theta\right)}{T^{2} R_{0}} \frac{\partial}{\partial \theta} R_{0}\left(1+\frac{r}{R_{0}} \cos \theta\right) \frac{\partial}{\partial \theta}\right] E_{r}+ \\
& {\left[\frac{\left(1-\frac{r}{R_{0}} \cos \theta\right)}{r^{2} R_{0}} \frac{\partial}{\partial \theta} R_{0}\left(1+\frac{r}{R_{0}} \cos \theta\right) \frac{\partial}{\partial r} \frac{r}{\sqrt{1+\delta^{2}}}\right.} \\
& \left.-\frac{\left(1-2 \frac{r}{R_{0}} \cos \theta\right)}{R_{0}^{2}} \frac{\partial}{\partial \zeta} \frac{\partial}{\partial r} \frac{\delta R_{0}\left(1+\frac{r}{R_{0}} \cos \theta\right)}{\sqrt{1+\delta^{2}}}\right] E_{\perp}+ \\
& {\left[\frac{\left(1-\frac{r}{R_{0}} \cos \theta\right)}{r^{2} R_{0}} \frac{\partial}{\partial \theta} R_{0}\left(1+\frac{r}{R_{0}} \cos \theta\right) \frac{\partial}{\partial r} \frac{\delta r}{\sqrt{1+\delta^{2}}}\right.} \\
& \left.+\frac{\left(1-2 \frac{r}{R_{0}} \cos \theta\right)}{R_{0}^{2}} \frac{\partial}{\partial \zeta} \frac{\partial}{\partial r} \frac{R_{0}\left(1+\frac{r}{R_{0}} \cos \theta\right)}{\sqrt{1+\delta^{2}}}\right] E_{t}
\end{aligned}
$$




$$
\begin{aligned}
& (\vec{\nabla} \times \vec{\nabla} \times \vec{E})_{r}= \\
& -\left[\frac{\left(1-2 \frac{r}{R_{0}} \cos \theta\right)}{R_{0}^{2}} \frac{\partial^{2}}{\partial \zeta^{2}}+\frac{\left(1-\frac{r^{2}}{R_{0}^{2}} \cos ^{2} \theta\right)}{r^{2}} \frac{\partial^{2}}{\partial \partial^{2}}-\frac{\left(1-\frac{r}{R_{0}} \cos \theta\right)}{r} \sin \theta \frac{\partial}{\partial \theta}\right] E_{r}+ \\
& {\left[\left(\frac{\left(1-\frac{r^{2}}{R_{0}^{2}} \cos ^{2} \theta\right)}{r^{2}} \frac{\partial}{\partial \theta}-\frac{\left(1-\frac{r}{R_{0}} \cos \theta\right) \sin \theta}{r R_{0}}\right) \frac{\partial}{\partial r} \frac{r}{\sqrt{1+\delta^{2}}}\right.} \\
& \left.-\frac{\left(1-2 \frac{r}{R_{0}} \cos \theta\right)}{R_{0}} \frac{\partial}{\partial r} \frac{\partial\left(1+\frac{r}{R_{0}} \cos \theta\right)}{\sqrt{1+\delta^{2}}} \frac{\partial}{\partial \zeta}\right] E_{1}+ \\
& {\left[\left(\frac{\left(1-\frac{r^{2}}{R_{0}^{2}} \cos ^{2} \theta\right)}{r^{2}} \frac{\partial}{\partial \theta}-\frac{\left(1-\frac{r}{R_{0}} \cos \theta\right) \sin \theta}{r R_{0}}\right) \frac{\partial}{\partial r} \frac{\delta r}{\sqrt{1+\delta^{2}}}\right.} \\
& \left.+\frac{\left(1-2 \frac{r}{R_{0}} \cos \theta\right)}{R_{0}^{2}} \frac{\partial}{\partial r} \frac{R_{0}\left(1+\frac{r}{R_{0}} \cos \theta\right)}{\sqrt{1+\delta^{2}}} \frac{\partial}{\partial \zeta}\right] E_{\|}
\end{aligned}
$$

$$
\begin{aligned}
& (\vec{\nabla} \times \vec{\nabla} \times \vec{E})_{r}= \\
& -\left[\frac{\left(1-2 \frac{r}{R_{0}} \cos \theta\right)}{R_{0}^{2}} \frac{\partial^{2}}{\partial \zeta^{2}}+\frac{\left(1-\frac{r^{2}}{R_{0}^{2}} \cos ^{2} \theta\right)}{r^{2}} \frac{\partial^{2}}{\partial \theta^{2}}-\frac{\left(1-\frac{r}{R_{0}} \cos \theta\right)}{r} \sin \theta \frac{\partial}{\partial \theta}\right] E_{r}+ \\
& {\left[\left(\frac{\left(1-\frac{r^{2}}{R_{r}^{2}} \cos ^{2} \theta\right)}{r^{2}} \frac{\partial}{\partial \theta}-\frac{\left(1-\frac{r}{R_{0}} \cos \theta\right) \sin \theta}{r R_{0}}\right) \frac{\partial}{\partial r} \frac{r}{\sqrt{1+\delta^{2}}}-\frac{\left(1-2 \frac{r}{R_{0}} \cos \theta\right)}{R_{0}} \times\right.} \\
& \left.\left(\frac{\delta}{\sqrt{1+\delta^{2}}} \frac{\cos \theta}{R_{\theta}}+\left(1+\frac{r}{R_{0}} \cos \theta\right)\left(\frac{\delta}{\sqrt{1+\delta^{2}}}\right)^{\prime}+\frac{\delta\left(1+\frac{r}{R_{0}} \cos \theta\right)}{\sqrt{1+\delta^{2}}} \frac{\partial}{\partial r}\right) \frac{\partial}{\partial \zeta}\right] E_{\perp}+ \\
& {\left[\left(\frac{\left(1-\frac{r^{2}}{R_{0}^{2}} \cos ^{2} \theta\right)}{r^{2}} \frac{\partial}{\partial \theta}-\frac{\left(1-\frac{r}{R_{0}} \cos \theta\right) \sin \theta}{r R_{\theta}}\right) \frac{\partial}{\partial r} \frac{\delta r}{\sqrt{1+\delta^{2}}}+\frac{\left(1-2 \frac{r}{R_{0}} \cos \theta\right)}{R_{0}^{2}} \times\right.} \\
& \left.\left(\frac{1}{\sqrt{1+\delta^{2}}} \frac{\cos \theta}{R_{0}}+\left(1+\frac{r}{R_{0}} \cos \theta\right)\left(\frac{1}{\sqrt{1+\delta^{2}}}\right)^{\prime}+\frac{\left(1+\frac{r}{R_{0}} \cos \theta\right)}{\sqrt{1+\delta^{2}}} \frac{\partial}{\partial r}\right) \frac{\partial}{\partial \zeta}\right] E_{1}
\end{aligned}
$$


$(\vec{\nabla} \times \vec{\nabla} \times \vec{E})_{r}=$

$-\left[\frac{1}{R_{0}^{2}} \frac{\partial^{2}}{\partial \zeta^{2}}+\frac{1}{T^{2}} \frac{\partial^{2}}{\partial \theta^{2}}-\frac{2 \frac{r}{R_{0}} \cos \theta}{R_{0}^{2}} \frac{\partial^{2}}{\partial \zeta^{2}}-\frac{\sin \theta}{r} \frac{\partial}{\partial \theta}-\frac{\cos ^{2} \theta}{R_{0}^{2}} \frac{\partial^{2}}{\partial \theta^{2}}+\frac{\cos \theta \sin \theta}{R_{0}} \frac{\partial}{\partial \theta}\right] E_{\mathrm{r}}+$

$\left[\left(\frac{1}{r^{2}} \frac{\partial}{\partial \theta}-\frac{\sin \theta-\frac{r}{R_{0}} \cos \theta \sin \theta}{r R_{\theta}}-\frac{\cos ^{2} \theta}{R_{0}^{2}} \frac{\partial}{\partial \theta}\right)\left(\left(\frac{r}{\sqrt{1+\delta^{2}}}\right)^{\prime}+\frac{r}{\sqrt{1+\delta^{2}}} \frac{\partial}{\partial r}\right)-\right.$

$\left.\frac{\left(1-2 \frac{r}{R_{0}} \cos \theta\right)}{R_{0}}\left(\left(1+\frac{r}{R_{0}} \cos \theta\right)\left(\frac{\delta}{\sqrt{1+\delta^{2}}}\right)^{\prime}+\frac{\delta\left(1+\frac{r}{R_{0}} \cos \theta\right)}{\sqrt{1+\delta^{2}}} \frac{\partial}{\partial r}+\frac{\delta}{\sqrt{1+\delta^{2}}} \cos \theta\right) \frac{\partial}{R_{0}}\right] E_{\perp}+$

$\left[\left(\frac{\left(1-\frac{r^{2}}{R_{\delta}^{2}} \cos ^{2} \theta\right)}{r^{2}} \frac{\partial}{\partial \theta}-\frac{\sin \theta-\frac{r}{R_{0}} \cos \theta \sin \theta}{r R_{0}}\right) \frac{\partial}{\partial r} \frac{\delta r}{\sqrt{1+\delta^{2}}}+\right.$

$\left.\frac{\left(1-2 \frac{r}{R_{0}} \cos \theta\right)}{R_{0}^{2}}\left(\left(1+\frac{r}{R_{0}} \cos \theta\right)\left(\frac{1}{\sqrt{1+\delta^{2}}}\right)^{\prime}+\frac{\left(1+\frac{r}{R_{0}} \cos \theta\right)}{\sqrt{1+\delta^{2}}} \frac{\partial}{\partial r}+\frac{1}{\sqrt{1+\delta^{2}}} \frac{\cos \theta}{R_{0}}\right) \frac{\partial}{\partial \zeta}\right] E_{\mathrm{H}}$

Desprezando termos proporcionais a segunda ordem em $\theta, \hat{e}$ alcançada a expressäo

$$
\begin{aligned}
& (\vec{\nabla} \times \vec{\nabla} \times \vec{E})_{r}=-\left[\frac{1}{R_{0}^{2}} \frac{\partial^{2}}{\partial \zeta^{2}}+\frac{1}{r^{2}} \frac{\partial^{2}}{\partial \theta^{2}}-\frac{2 \frac{r}{R_{0}} \cos \theta}{R_{0}^{2}} \frac{\partial^{2}}{\partial \zeta^{2}}-\frac{\sin \theta}{r} \frac{\partial}{\partial \theta}\right] E_{r}+ \\
& {\left[\left(\frac{1}{r^{2}} \frac{\partial}{\partial \theta}-\frac{\sin \theta}{r R_{0}}\right)\left(\left(\frac{r}{\sqrt{1+\delta^{2}}}\right)^{\prime}+\frac{r}{\sqrt{1+\delta^{2}}} \frac{\partial}{\partial r}\right)+\right.} \\
& \frac{2 \frac{r}{R_{0}} \cos \theta}{R_{0}}\left\{\left(\frac{\delta}{\sqrt{1+\delta^{2}}}\right)^{\prime}+\frac{\delta}{\sqrt{1+\delta^{2}}} \frac{\partial}{\partial r}+\frac{\partial}{\partial \zeta}\right\} \frac{\partial}{\partial \zeta} \\
& \left.-\frac{1}{R_{0}}\left(\left(1+\frac{r}{R_{0}} \cos \theta\right)\left(\frac{\delta}{\sqrt{1+\delta^{2}}}\right)^{\prime}+\frac{\delta\left(1+\frac{r}{R_{0}} \cos \theta\right)}{\sqrt{1+\delta^{2}}} \frac{\partial}{\partial r}+\frac{\delta}{\sqrt{1+\delta^{2}}} \frac{\cos \theta}{R_{0}}\right)\right] E_{\perp}+ \\
& {\left[\left(\frac{1}{r^{2}} \frac{\partial}{\partial \theta}-\frac{\sin \theta}{r R_{0}}\right) \frac{\partial}{\partial r} \frac{\delta r}{\sqrt{1+\delta^{2}}}-\frac{2 \frac{r}{R_{0}} \cos \theta}{R_{0}^{2}}\left\{\left(\frac{1}{\sqrt{1+\delta^{2}}}\right)^{\prime}+\frac{1}{\sqrt{1+\delta^{2}}} \frac{\partial}{\partial r}\right\}\right.} \\
& \left.+\frac{1}{R_{0}^{2}}\left\{\left(1+\frac{r}{R_{0}} \cos \theta\right)\left(\frac{1}{\sqrt{1+\delta^{2}}}\right)^{\prime}+\frac{\left(1+\frac{r}{R_{0}} \cos \theta\right)}{\sqrt{1+\delta^{2}}} \frac{\partial}{\partial r}+\frac{1}{\sqrt{1+\delta^{2}}} \frac{\cos \theta}{R_{0}}\right\} \frac{\partial}{\partial \zeta}\right] E_{(193)}
\end{aligned}
$$




\section{E. 2.2 Componente perpendicular}

$$
\begin{aligned}
& (\vec{\nabla} \times \vec{\nabla} \times \vec{E})_{1}=\left[\frac{1}{R \sqrt{1+\delta^{2}}} \frac{\partial}{\partial r} \frac{R}{r} \frac{\partial}{\partial \theta}-\frac{\partial}{r \sqrt{1+\delta^{2}}} \frac{\partial}{\partial r} \frac{r}{R} \frac{\partial}{\partial \zeta}\right] E_{r}- \\
& {\left[\frac{1}{R \sqrt{1+\delta^{2}}}\left(\frac{\partial}{\partial \zeta}+\frac{\delta R}{r} \frac{\partial}{\partial \theta}\right) \frac{1}{R}\left(\frac{\partial}{\partial \zeta}+\frac{1}{r} \frac{\partial}{\partial \theta} \delta R\right) \frac{1}{\sqrt{1+\delta^{2}}}+\right.} \\
& \left.\frac{1}{R \sqrt{1+\delta^{2}}} \frac{\partial R}{\partial r} \frac{\partial}{\partial r} \frac{r}{\sqrt{1+\delta^{2}}}+\frac{\delta}{r \sqrt{1+\delta^{2}}} \frac{\partial}{\partial r} \frac{r}{R} \frac{\partial}{\partial r} \frac{\delta R}{\sqrt{1+\delta^{2}}}\right] E_{L}+ \\
& {\left[\frac{1}{R \sqrt{1+\delta^{2}}}\left(\frac{\partial}{\partial \zeta}+\frac{\delta R}{r} \frac{\partial}{\partial \theta}\right) \frac{1}{R}\left(\frac{\delta}{R} \frac{\partial}{\partial \zeta}-\frac{1}{r} \frac{\partial}{\partial \theta}\right) \frac{R}{\sqrt{1+\delta^{2}}}+\right.} \\
& \left.\frac{1}{R \sqrt{1+\delta^{2}}} \frac{\partial}{\partial r} \frac{R}{\partial r} \frac{\partial \delta}{\sqrt{1+\delta^{2}}}-\frac{\delta}{r \sqrt{1+\delta^{2}}} \frac{\partial r}{\partial r} \frac{\partial}{R r} \frac{R}{\sqrt{1+\delta^{2}}}\right] E_{\text {II }}(194) \\
& (\vec{\nabla} \times \vec{\nabla} \times \vec{E})_{1}=\left[\frac{1-\frac{r}{R_{0}} \cos \theta}{\sqrt{1+\delta^{2}}} \frac{\partial}{\partial r} \frac{1+\frac{r}{R_{0}} \cos \theta}{r} \frac{\partial}{\partial \theta}-\frac{\delta}{r \sqrt{1+\delta^{2}}} \frac{\partial}{\partial r} \frac{r\left(1-\frac{r}{R_{0}} \cos \theta\right)}{R_{0}} \frac{\partial}{\partial \zeta}\right] E_{r}- \\
& {\left[\frac { 1 - \frac { r } { R _ { 0 } } \operatorname { c o s } \theta } { R _ { 0 } \sqrt { 1 + \delta ^ { 2 } } } ( \frac { \partial } { \partial \zeta } + \frac { \delta R } { r } \frac { \partial } { \partial \theta } ) \frac { 1 } { R } \left(\frac{\partial}{\partial \zeta}+\frac{\delta}{r \partial \theta} R_{0}\left(1+\frac{r}{R_{0}} \cos \theta\right) \frac{1}{\sqrt{1+\delta^{2}}}+\right.\right.} \\
& \left.\frac{1-\frac{r}{R_{0}} \cos \theta}{\sqrt{1+\delta^{2}}} \frac{\partial}{\partial r} \frac{1+\frac{r}{R_{0}} \cos \theta}{r} \frac{\partial}{\partial r} \frac{r}{\sqrt{1+\delta^{2}}}+\frac{\delta}{r \sqrt{1+\delta^{2}}} \frac{\partial}{\partial r} r\left(1-\frac{r}{R_{0}} \cos \theta\right) \frac{\partial}{\partial r} \frac{\delta\left(1+\frac{r}{R_{0}} \cos \theta\right)}{\sqrt{1+\delta^{2}}}\right] E_{\perp}+ \\
& {\left[\frac { 1 - \frac { x } { R _ { 0 } } \operatorname { c o s } \theta } { R _ { 0 } \sqrt { 1 + \delta ^ { 2 } } } ( \frac { \partial } { \partial \zeta } + \frac { \delta R } { r } \frac { \partial } { \partial \theta } ) \frac { 1 } { R } \left(\delta \frac{\partial}{\partial \zeta}-\frac{1}{r} \frac{\partial}{\partial \theta} R_{0}\left(1+\frac{r}{R_{0}} \cos \theta\right) \frac{1}{\sqrt{1+\delta^{2}}}+\right.\right.} \\
& \left.\frac{1-\frac{r}{R_{0}} \cos \theta}{\sqrt{1+\delta^{2}}} \frac{\partial}{\partial r} \frac{1+\frac{r}{R_{0}} \cos \theta}{r} \frac{\partial}{\partial r} \frac{r \delta}{\sqrt{1+\delta^{2}}}-\frac{\delta}{r \sqrt{1+\delta^{2}}} \frac{\partial}{\partial r} r\left(1-\frac{r}{R_{0}} \cos \theta\right) \frac{\partial}{\partial r} \frac{1+\frac{r}{R_{0}} \cos \theta}{\sqrt{1+\delta^{2}}}\right] E_{1}
\end{aligned}
$$


$(\vec{\nabla} \times \vec{\nabla} \times \vec{E})_{1}=$

$\left[\frac{1-\frac{r}{R_{0}} \cos \theta}{\sqrt{1+\delta^{2}}} \frac{\partial}{\partial r} \frac{1+\frac{r}{R_{0}} \cos \theta}{r} \frac{\partial}{\partial \theta}-\frac{\delta}{r \sqrt{1+\delta^{2}}} \frac{\partial r\left(1-\frac{x}{R_{0}} \cos \theta\right)}{R_{0}} \frac{\partial}{\partial \zeta}\right] E_{r}-$

$\left[\frac{1-\frac{r}{R_{0}} \cos \theta}{R_{0} \sqrt{1+\delta^{2}}}\left(\frac{1-\frac{r}{R_{0}} \cos \theta}{R_{0}} \frac{\partial}{\partial \zeta}+\frac{\partial \partial}{r \partial \theta}+\frac{\delta R}{r}\left(-\frac{1}{R^{2}}\right)\left(\frac{\partial}{\partial \theta} R\right)\right) \times\right.$

$\left(\frac{\partial}{\partial \zeta}+\frac{\delta R_{0}\left(1+\frac{r}{\phi_{0}} \cos \theta\right)}{r} \frac{\partial}{\partial \theta}-\delta \sin \theta\right) \frac{1}{\sqrt{1+\delta^{2}}}+$

$\left.\frac{1-\frac{r}{R_{0}} \cos \theta}{\sqrt{1+\delta^{2}}} \frac{\partial}{\partial r} \frac{1+\frac{r}{R_{0}} \cos \theta}{r} \frac{\partial}{\partial r} \frac{r}{\sqrt{1+\delta^{2}}}+\frac{\delta}{r \sqrt{1+\delta^{2}}} \frac{\partial}{\partial r} r\left(1-\frac{r}{R_{0}} \cos \theta\right) \frac{\partial}{\partial r} \frac{\delta\left(1+\frac{r}{R_{0}} \cos \theta\right)}{\sqrt{1+\delta^{2}}}\right] E_{\perp}+$

$\left[\frac{1-\frac{r}{R_{0}} \cos \theta}{R_{0} \sqrt{1+\delta^{2}}}\left(\frac{1-\frac{r}{R_{0}} \cos \theta}{R_{0}} \frac{\partial}{\partial \zeta}+\frac{\delta}{r} \frac{\partial}{\partial \theta}+\frac{\delta R}{r}\left(-\frac{1}{R^{2}}\right)\left(\frac{\partial}{\partial \theta} R\right)\right) \times\right.$

$\left(\delta \frac{\partial}{\partial \zeta}-\frac{R_{0}\left(1+\frac{r}{R_{0}} \cos \theta\right)}{r} \frac{\partial}{\partial \theta}+\sin \theta\right) \frac{1}{\sqrt{1+\delta^{2}}}+$

$\left.\frac{1-\frac{r}{R_{0}} \cos \theta}{\sqrt{1+\delta^{2}}} \frac{\partial}{\partial r} \frac{1+\frac{r}{R_{0}} \cos \theta}{r} \frac{\partial}{\partial r} \frac{r \delta}{\sqrt{1+\delta^{2}}}-\frac{\delta}{r \sqrt{1+\delta^{2}}} \frac{\partial}{\partial r} r\left(1-\frac{r}{R_{0}} \cos \theta\right) \frac{\partial}{\partial r} \frac{1+\frac{r}{R_{0}} \cos \theta}{\sqrt{1+\delta^{2}}}\right] E_{n}$

$(\vec{\nabla} \times \vec{\nabla} \times \vec{E})_{\perp}=\left[\frac{1-\frac{r}{R_{0}} \cos \theta}{\sqrt{1+\delta^{2}}} \frac{\partial}{\partial r} \frac{1+\frac{r}{R_{0}} \cos \theta}{r} \frac{\partial}{\partial \theta}-\frac{\delta}{r \sqrt{1+\delta^{2}}} \frac{\partial}{\partial r} \frac{r}{R} \frac{\partial}{\partial \zeta}\right] E_{r}-$

$\left[\frac{1-\frac{r}{R_{0}} \cos \theta}{R_{0}\left(1+\delta^{2}\right)}\left(\frac{1-\frac{r}{R_{0}} \cos \theta}{R_{0}} \frac{\partial}{\partial \zeta}+\frac{\delta}{r \partial \theta}+\frac{\delta}{R} \sin \theta\right)\left(\frac{\partial}{\partial \zeta}+\frac{\delta R_{0}\left(1+\frac{r}{R_{0}} \cos \theta\right)}{r} \frac{\partial}{\partial \theta}-\delta \sin \theta\right)+\right.$

$\left.\frac{1-\frac{r}{R_{0}} \cos \theta}{\sqrt{1+\delta^{2}} \frac{\partial}{\partial r}} \frac{1+\frac{r}{R_{0}} \cos \theta}{r} \frac{\partial}{\partial r} \frac{r}{\sqrt{1+\delta^{2}}}+\frac{\delta}{r \sqrt{1+\delta^{2}}} \frac{\partial}{\partial r} r\left(1-\frac{r}{R_{0}} \cos \theta\right) \frac{\partial}{\partial r} \frac{\delta\left(1+\frac{r}{R_{R^{2}}} \cos \theta\right)}{\sqrt{1+\delta^{2}}}\right] E_{1}+$

$\left[\frac{1-\frac{t_{0}}{R_{0}} \cos \theta}{R_{0}\left(1+\delta^{2}\right)}\left(\frac{1-\frac{r}{R_{0}} \cos \theta}{R_{0}} \frac{\partial}{\partial \zeta}+\frac{\delta}{r} \frac{\partial}{\partial \theta}+\frac{\delta}{R} \sin \theta\right)\left(\delta \frac{\partial}{\partial \zeta}-\frac{R_{0}\left(1+\frac{r}{R_{0}} \cos \theta\right)}{r} \frac{\partial}{\partial \theta}+\sin \theta\right)+\right.$

$\left.\frac{1-\frac{r}{R_{0}} \cos \theta}{\sqrt{1+\delta^{2}}} \frac{\partial}{\partial r} \frac{1+\frac{r}{R_{0}} \cos \theta}{r} \frac{\partial}{\partial r} \frac{r \delta}{\sqrt{1+\delta^{2}}}-\frac{\delta}{r \sqrt{1+\delta^{2}}} \frac{\partial}{\partial r} r\left(1-\frac{r}{R_{0}} \cos \theta\right) \frac{\partial}{\partial r} \frac{1+\frac{r}{R_{0}} \cos \theta}{\sqrt{1+\delta^{2}}}\right] E_{\|}$

$(\vec{\nabla} \times \vec{\nabla} \times \vec{E})_{\perp}=\left[\frac{1-\frac{r}{R_{0}} \cos \theta}{\sqrt{1+\delta^{2}}}\left(\frac{1+\frac{r}{R_{0}} \cos \theta}{r} \frac{\partial}{\partial r} \frac{\partial}{\partial \theta}-\frac{1}{r^{2}} \frac{\partial}{\partial \theta}\right)-\right.$

$\left.\frac{\delta}{\sqrt{1+\delta^{2}}}\left(\frac{\left(1-\frac{r}{R_{0}} \cos \theta\right)}{R_{0}} \frac{\partial}{\partial r} \frac{\partial}{\partial \zeta}+\frac{\left(1-\frac{r}{R_{0}} \cos \theta\right)}{r R_{0}} \frac{\partial}{\partial \zeta}-\frac{\left(1-2 \frac{r}{R_{0}} \cos \theta\right) \cos \theta}{R_{0}^{2}} \frac{\partial}{\partial \zeta}\right)\right] E_{\mathrm{r}}-$

$\left[\frac{1-\frac{r}{R_{0}} \cos \theta}{R_{0}\left(1+\delta^{2}\right)}\left(\frac{1-\frac{r}{R_{0}} \cos \theta}{R_{0}} \frac{\partial}{\partial \zeta}+\frac{\delta}{r} \frac{\partial}{\partial \theta}+\frac{\delta}{R} \sin \theta\right)\left(\frac{\partial}{\partial \zeta}+\frac{\delta R_{0}\left(1+\frac{\pi}{R_{0}} \cos \theta\right)}{r} \frac{\partial}{\partial \theta}-\delta \sin \theta\right)+\right.$

$\left.\frac{1-\frac{r}{R_{0}} \cos \theta}{\sqrt{1+\delta^{2}}} \frac{\partial}{\partial r} \frac{1+\frac{r}{R_{0}} \cos \theta}{r} \frac{\partial}{\partial r} \frac{r}{\sqrt{1+\delta^{2}}}+\frac{\delta}{r \sqrt{1+\delta^{2}}} \frac{\partial}{\partial r} r\left(1-\frac{r}{R_{0}} \cos \theta\right) \frac{\partial}{\partial r} \frac{\delta\left(1+\frac{r}{R_{0}} \cos \theta\right)}{\sqrt{1+\delta^{2}}}\right] E_{\perp}+$

$\left[\frac{1-\frac{r}{R_{0}} \cos \theta}{R_{0}\left(1+\delta^{2}\right)}\left(\frac{1-\frac{r}{R_{0}} \cos \theta}{R_{0}} \frac{\partial}{\partial \zeta}+\frac{\delta}{r} \frac{\partial}{\partial \theta}+\frac{\delta}{R} \sin \theta\right)\left(\delta \frac{\partial}{\partial \zeta}-\frac{R_{0}\left(1+\frac{r}{R_{0}} \cos \theta\right)}{r} \frac{\partial}{\partial \theta}+\sin \theta\right)+\right.$

$\left.\frac{1-\frac{r}{R_{0}} \cos \theta}{\sqrt{1+\delta^{2}}} \frac{\partial}{\partial r} \frac{1+\frac{r}{R_{0}} \cos \theta}{r} \frac{\partial}{\partial r} \frac{r \delta}{\sqrt{1+\delta^{2}}}-\frac{\delta}{r \sqrt{1+\delta^{2}}} \frac{\partial}{\partial r} r\left(1-\frac{r}{R_{0}} \cos \theta\right) \frac{\partial}{\partial r} \frac{1+\frac{r}{R_{0}} \cos \theta}{\sqrt{1+\delta^{2}}}\right] E_{\|}$ 
Desprezando os termos proponclonais à segunda ordem em $\theta$;

$$
\begin{aligned}
& (\vec{\nabla} \times \vec{\nabla} \times \vec{E})_{L}= \\
& \frac{1}{\sqrt{1+\delta^{2}}}\left[\frac{1}{r} \frac{\partial}{\partial r} \frac{\partial}{\partial \theta}-\frac{1-\frac{r}{\delta_{0}} \cos \theta}{r^{2}} \frac{\partial}{\partial \theta}-\frac{\delta\left(1-\frac{r}{R_{0}} \cos \theta\right)}{R_{0}} \frac{\partial}{\partial r} \frac{\partial}{\partial \zeta}-\frac{\delta\left(1-2 \frac{r}{\hat{R}_{0}} \cos \theta\right)}{r R_{0}} \frac{\partial}{\partial \zeta}\right] E_{r} \\
& -\left[\frac{1-\frac{r}{R_{0}} \cos \theta}{R_{0}\left(1+\delta^{2}\right)}\left(\frac{1-\frac{r}{R_{0}} \cos \theta}{R_{0}} \frac{\partial}{\partial \zeta}+\frac{\delta}{r} \frac{\partial}{\partial \theta}+\frac{\delta}{R} \sin \theta\right)\left(\frac{\partial}{\partial \zeta}+\frac{\delta R_{0}\left(1+\frac{r}{R_{0}}\right.}{r} \cos \theta\right) \frac{\partial}{\partial \theta}-\delta \sin \theta\right) \\
& +\frac{1-\frac{r}{\delta_{0}} \cos \theta}{\sqrt{1+\delta^{2}}}\left(\frac{\partial}{\partial r} \frac{1}{r^{2}} \frac{\partial}{\partial r}+\frac{\cos \theta}{R_{0}} \frac{\partial^{2}}{\partial r^{2}}\right) \frac{r}{\sqrt{1+\delta^{2}}} \\
& \left.+\frac{\delta}{r \sqrt{1+\delta^{2}}}\left\{\left(1-\frac{r}{R_{0}} \cos \theta\right) \frac{\partial}{\partial r} r \frac{\partial}{\partial r}-\frac{r}{R_{0}} \cos \theta \frac{\partial}{\partial r}\right\} \frac{\delta\left(1+\frac{r}{R_{0}} \cos \theta\right)}{\sqrt{1+\delta^{2}}}\right] E_{1} \\
& +\left[\frac{1-\frac{r}{R_{0}} \cos \theta}{R_{0}\left(1+\delta^{2}\right)}\left(\frac{1-\frac{r}{R_{0}} \cos \theta}{R_{0}} \frac{\partial}{\partial \zeta}+\frac{\delta}{r} \frac{\partial}{\partial \theta}+\frac{\delta}{R} \sin \theta\right)\left(\delta \frac{\partial}{\partial \zeta}-\frac{R_{0}\left(1+\frac{r}{R_{0}} \cos \theta\right)}{r} \frac{\partial}{\partial \theta}+\sin \theta\right)\right. \\
& +\frac{1-\frac{r}{\xi_{0}} \cos \theta}{\sqrt{1+\delta^{2}}}\left\{\frac{\partial}{\partial r} \frac{1}{r} \frac{\partial}{\partial r}+\frac{\cos \theta}{R_{0}} \frac{\partial^{2}}{\partial r^{2}}\right\} \frac{r \delta}{\sqrt{1+\delta^{2}}} \\
& \left.-\frac{\delta}{r \sqrt{1+\delta^{2}}}\left\{\left(1-\frac{r}{R_{0}} \cos \theta\right) \frac{\partial}{\partial r} r \frac{\partial}{\partial r}-\frac{r}{R_{0}} \cos \theta \frac{\partial}{\partial r}\right\} \frac{1+\frac{r}{R_{0}} \cos \theta}{\sqrt{1+\delta^{2}}}\right] E_{\|} \\
& (\vec{\nabla} \times \vec{\nabla} \times \vec{E})_{1}= \\
& \frac{1}{\sqrt{1+\delta^{2}}}\left[\frac{1}{r} \frac{\partial}{\partial r} \frac{\partial}{\partial \theta}-\frac{1-\frac{r}{R_{0}} \cos \theta}{r^{2}} \frac{\partial}{\partial \theta}-\frac{\delta\left(1-\frac{r}{R_{0}} \cos \theta\right)}{R_{0}} \frac{\partial}{\partial r} \frac{\partial}{\partial \zeta}-\frac{\delta\left(1-2 \frac{r}{R_{0}} \cos \theta\right)}{r R_{0}} \frac{\partial}{\partial \zeta}\right] E_{r} \\
& -\left[\frac{1-2 \frac{r}{h_{\theta}} \cos \theta}{R\left(1+\delta^{2}\right)}\left(\frac{\partial}{\partial \zeta}+\frac{\delta R}{r} \frac{\partial}{\partial \theta}+\delta \sin \theta\right)\left(\frac{\partial}{\partial \zeta}+\frac{\delta R \partial}{r \partial}-\delta \sin \theta\right)\right. \\
& +\left(\frac{1-\frac{r}{R_{0}} \cos \theta}{\sqrt{1+\delta^{2}}} \frac{\partial}{\partial r} \frac{1}{r} \frac{\partial}{\partial r}+\frac{\cos \theta}{R_{0} \sqrt{1+\delta^{2}}} \frac{\partial^{2}}{\partial r^{2}}\right) \frac{r}{\sqrt{1+\delta^{2}}} \\
& \left.+\frac{\partial}{r \sqrt{1+\delta^{2}}}\left(\left(1-\frac{r}{R_{0}} \cos \theta\right) \frac{\partial}{\partial r} r \frac{\partial}{\partial r}-\frac{r}{R_{0}} \cos \theta \frac{\partial}{\partial r}\right) \frac{\delta\left(1+\frac{r}{R_{0}} \cos \theta\right)}{\sqrt{1+\delta^{2}}}\right] E_{1} \\
& +\left[\frac{1-2 \frac{r}{R_{0}} \cos \theta}{R_{0}^{2}\left(1+\delta^{2}\right)}\left(\frac{\partial}{\partial \zeta}+\frac{\delta R}{r} \frac{\partial}{\partial \theta}+\delta \sin \theta\right)\left(\delta \frac{\partial}{\partial \zeta}-\frac{R}{\partial \theta}+\sin \theta\right)\right. \\
& +\left\{\frac{1-\frac{r}{R_{0}} \cos \theta}{\sqrt{1+\delta^{2}}} \frac{\partial}{\partial r} \frac{1}{\partial r}+\frac{\cos \theta}{R_{0} \sqrt{1+\delta^{2}}} \frac{\partial^{2}}{\partial r^{2}}\right\} \frac{r \delta}{\sqrt{1+\delta^{2}}} \\
& \left.-\frac{\delta}{r \sqrt{1+\delta^{2}}}\left\{\left(1-\frac{r}{R_{0}} \cos \theta\right) \frac{\partial}{\partial r} r \frac{\partial}{\partial r}-\frac{r^{2}}{R_{0}} \cos \theta \frac{\partial}{\partial r}\right) \frac{1+\frac{r}{n_{0}} \cos \theta}{\sqrt{1+\delta^{2}}}\right] g_{1}
\end{aligned}
$$


$(\vec{\nabla} \times \vec{\nabla} \times \vec{E})_{1}=$

$\frac{1}{\sqrt{1+\delta^{2}}}\left[\frac{1}{r} \frac{\partial}{\partial r} \frac{\partial}{\partial \theta}-\frac{1-\frac{r}{R_{0}} \cos \theta}{r^{2}} \frac{\partial}{\partial \theta}-\frac{\delta\left(1-\frac{r}{R_{0}} \cos \theta\right)}{R_{L_{0}}} \frac{\partial}{\partial r} \frac{\partial}{\partial \zeta}-\frac{\delta\left(1-2 \frac{r}{R_{0}} \cos \theta\right)}{r R_{0}} \frac{\partial}{\partial \zeta}\right] E_{r}$

$-\left[\frac{1-2 \frac{r}{R_{0}} \cos \theta}{R_{0}^{2}\left(1+\delta^{2}\right)}\left(\frac{\partial}{\partial \zeta}+\frac{1}{q} \frac{\partial}{\partial \theta}+\delta \cos \theta \frac{\partial}{\partial \theta}+\delta \sin \theta\right)\left(\frac{\partial}{\partial \zeta}+\frac{1}{q} \frac{\partial}{\partial \theta}+\delta \cos \theta \frac{\partial}{\partial \theta}-\delta \sin \theta\right)\right.$

$+\left(\frac{1-\frac{r}{R_{0}} \cos \theta}{\sqrt{1+\delta^{2}}} \frac{\partial}{\partial r} \frac{1}{\partial r} \frac{\partial}{\partial r}+\frac{\cos \theta}{R_{0} \sqrt{1+\delta^{2}}} \frac{\partial^{2}}{\partial r^{2}}\right) \frac{r}{\sqrt{1+\delta^{2}}}$

$\left.+\frac{\delta}{r \sqrt{1+\delta^{2}}}\left\{\frac{\partial}{\partial r} r \frac{\partial}{\partial r}+\frac{\cos \theta}{R_{0}} \frac{\partial}{\partial r} r \frac{\partial}{\partial r} r-\frac{r}{R_{0}} \cos \theta \frac{\partial}{\partial r} r \frac{\partial}{\partial r}-\frac{r}{R_{0}} \cos \theta \frac{\partial}{\partial r}\right\} \frac{\delta}{\sqrt{1+\delta^{2}}}\right] E_{\perp}$

$+\left[\frac{1-2 \frac{\tau}{R_{0}} \cos \theta}{R_{0}^{2}\left(1+\delta^{2}\right)}\left(\frac{\partial}{\partial \zeta}+\frac{1}{q} \frac{\partial}{\partial \theta}+\delta \cos \theta \frac{\partial}{\partial \theta}+\delta \sin \theta\right)\left(\delta \frac{\partial}{\partial \zeta}-\frac{R_{0}\left(1+\frac{r}{R_{0}} \cos \theta\right)}{r} \frac{\partial}{\partial \theta}+\sin \theta\right)\right.$

$+\left\{\frac{1-\frac{r}{R_{6}} \cos \theta}{\sqrt{1+\delta^{2}}} \frac{\partial}{\partial r} \frac{1}{r} \frac{\partial}{\partial r}+\frac{\cos \theta}{R_{0} \sqrt{1+\delta^{2}}} \frac{\partial^{2}}{\partial r^{2}}\right\} \frac{r \delta}{\sqrt{1+\delta^{2}}}$

$\left.-\frac{\delta}{r \sqrt{1+\delta^{2}}}\left\{\frac{\partial}{\partial r} r \frac{\partial}{\partial r}+\frac{\cos \theta}{R_{0}} \frac{\partial}{\partial r} r \frac{\partial}{\partial r} r-\frac{r}{R_{0}} \cos \theta \frac{\partial}{\partial r} r \frac{\partial}{\partial r}-\frac{r}{R_{0}} \cos \theta \frac{\partial}{\partial r}\right\} \frac{1}{\sqrt{1+\delta^{2}}}\right] E_{\|}$

$(\vec{\nabla} \times \vec{\nabla} \times \vec{E})_{2}=$

$\frac{1}{\sqrt{1+\delta^{2}}}\left[\frac{1}{r} \frac{\partial}{\partial r} \frac{\partial}{\partial \theta}-\frac{1-\frac{r}{R_{0}} \cos \theta}{r^{2}} \frac{\partial}{\partial \theta}-\frac{\delta\left(1-\frac{r}{R_{0}} \cos \theta\right)}{R_{0}} \frac{\partial}{\partial r} \frac{\partial}{\partial \zeta}-\frac{\delta\left(1-2 \frac{r}{R_{0}} \cos \theta\right)}{r R_{0}} \frac{\partial}{\partial \zeta}\right] E_{r}$

$-\left[\frac{1-2 \frac{r}{R_{0}} \cos \theta}{R_{0}^{2}\left(1+\delta^{2}\right)}\left(\frac{\partial}{\partial \zeta}+\frac{1}{q} \frac{\partial}{\partial \theta}+\delta \cos \theta \frac{\partial}{\partial \theta}+\delta \sin \theta\right)\left(\frac{\partial}{\partial \zeta}+\frac{1}{q} \frac{\partial}{\partial \theta}+\delta \cos \theta \frac{\partial}{\partial \theta}-\delta \sin \theta\right)\right.$

$+\left(\frac{1-\frac{r}{R_{0}} \cos \theta}{\sqrt{1+\delta^{2}}} \frac{\partial}{\partial r} \frac{1}{r} \frac{\partial}{\partial r}+\frac{\cos \theta}{R_{0} \sqrt{1+\delta^{2}}} \frac{\partial^{2}}{\partial r^{2}}\right) \frac{r}{\sqrt{1+\delta^{2}}}$

$\left.+\frac{\delta}{r \sqrt{1+\delta^{2}}}\left\{\frac{\partial}{\partial r} r \frac{\partial}{\partial r}+\frac{\cos \theta}{R_{w_{0}}} \frac{\partial}{\partial r} r \frac{\partial}{\partial r} r-\frac{r}{R_{0}} \cos \theta \frac{\partial}{\partial r} r \frac{\partial}{\partial r}-\frac{r}{R_{0}} \cos \theta \frac{\partial}{\partial r}\right\} \frac{\delta}{\sqrt{1+\delta^{2}}}\right] E_{\perp}$

$+\left[\frac{1-2 \frac{r}{R_{0}} \cos \theta}{R_{0}^{2}\left(1+\delta^{2}\right)}\left(\frac{\partial}{\partial \zeta}+\frac{1}{q} \frac{\partial}{\partial \theta}+\delta \cos \theta \frac{\partial}{\partial \theta}+\delta \sin \theta\right)\left(\delta \frac{\partial}{\partial \zeta}-\frac{R_{0}}{r} \frac{\partial}{\partial \theta}-\cos \theta \frac{\partial}{\partial \theta}+\sin \theta\right)\right.$

$+\left\{\frac{1-\frac{r}{R_{0}} \cos \theta}{\sqrt{1+\delta^{2}}} \frac{\partial}{\partial r} \frac{1}{r} \frac{\partial}{\partial r}+\frac{\cos \theta}{R_{0} \sqrt{1+\delta^{2}}} \frac{\partial^{2}}{\partial r^{2}}\right\} \frac{r \delta}{\sqrt{1+\delta^{2}}}$

$\left.-\frac{\delta}{r \sqrt{1+\delta^{2}}}\left\{\frac{\partial}{\partial r} r \frac{\partial}{\partial r}+\frac{\cos \theta}{R_{0}} \frac{\partial}{\partial r} r \frac{\partial}{\partial r} r-\frac{r}{R_{0}} \cos \theta \frac{\partial}{\partial r} r \frac{\partial}{\partial r}-\frac{r}{R_{0}} \cos \theta \frac{\partial}{\partial r}\right\} \frac{1}{\sqrt{1+\delta^{2}}}\right] E_{\|}$ 
Fazendo a simplificação

$$
\begin{aligned}
& \left(\frac{\partial}{\partial \zeta}+\frac{1}{q} \frac{\partial}{\partial \theta}+\delta \cos \theta \frac{\partial}{\partial \theta}+\delta \sin \theta\right)\left(\frac{\partial}{\partial \zeta}+\frac{1}{\partial \partial} \frac{\partial}{\partial \theta}+\delta \cos \theta \frac{\partial}{\partial \theta}-\delta \sin \theta\right)=\left(\frac{\partial}{\partial \zeta}+\frac{1}{\partial} \frac{\partial}{\partial \theta}\right)^{2}+ \\
& \left(\frac{\partial}{\partial \zeta}+\frac{1}{q} \frac{\partial}{\partial \theta}\right)\left(\delta \cos \theta \frac{\partial}{\partial \theta}-\delta \sin \theta\right)+\left(\delta \cos \theta \frac{\partial}{\partial \theta}+\delta \sin \theta\right)\left(\frac{\partial}{\partial \zeta}+\frac{1}{\partial} \frac{\partial}{\partial \theta}\right) \\
& +\left(\delta \cos \theta \frac{\partial}{\partial \theta}-\delta \sin \theta\right)\left(\delta \cos \theta \frac{\partial}{\partial \theta}+\delta \sin \theta\right) \text { Estes últimos termos serão desprezados... } \\
& =\left(\frac{\partial}{\partial \zeta}+\frac{1}{q \partial \theta}\right)^{2}+\left(\delta \cos \theta \frac{\partial}{\partial \theta}-\delta \sin \theta\right) \frac{\partial}{\partial \zeta}+\frac{1}{\partial \partial \theta}\left(\delta \cos \theta \frac{\partial}{\partial \theta}-\delta \sin \theta\right)+\delta \sin \theta\left(\frac{\partial}{\partial \zeta}+\frac{1}{q} \frac{\partial}{\partial \theta}\right) \\
& +\delta \cos \theta \frac{\partial}{\partial \theta}\left(\frac{\partial}{\partial \zeta}+\frac{1}{q} \frac{\partial}{\partial \theta}\right)=\left(\frac{\partial}{\partial \zeta}+\frac{1}{q} \frac{\partial}{\partial \theta}\right)^{2}+\left(\delta \cos \theta \frac{\partial}{\partial \theta}-\delta \sin \theta\right) \frac{\partial}{\partial \zeta}+ \\
& \frac{1}{q}\left(\delta \cos \theta \frac{\partial^{2}}{\partial \theta^{2}}-2 \delta \sin \theta \frac{\partial}{\partial \theta}-\delta \cos \theta\right)+\delta \sin \theta\left(\frac{\partial}{\partial \zeta}+\frac{1}{q} \frac{\partial}{\partial \theta}\right)+\left(\delta \cos \theta \frac{\partial}{\partial \theta} \frac{\partial}{\partial \zeta}+\frac{1}{q} \delta \cos \theta \frac{\partial^{2}}{\partial \theta^{2}}\right) \\
& =\left(\frac{\partial}{\partial \zeta}+\frac{1}{q} \frac{\partial}{\partial \theta}\right)^{2}+2 \delta \cos \theta \frac{\partial}{\partial \theta} \frac{\partial}{\partial \zeta}+\frac{1}{q}\left(2 \delta \cos \theta \frac{\partial^{2}}{\partial \theta^{2}}-\delta \sin \theta \frac{\partial}{\partial \theta}-\delta \cos \theta\right) \\
& =\left(\frac{\partial}{\partial \zeta}+\frac{1}{q} \frac{\partial}{\partial \theta}\right)^{2}+2 \delta \cos \theta \frac{\partial}{\partial \theta}\left(\frac{\partial}{\partial \zeta}+\frac{1}{q} \frac{\partial}{\partial \theta}\right)-\frac{1}{\eta}\left(\delta \sin \theta \frac{\partial}{\partial \theta}+\delta \cos \theta\right), \\
& \left(\frac{\partial}{\partial \zeta}+\frac{1}{\partial \theta} \frac{\partial}{\partial \theta}+\delta \cos \theta \frac{\partial}{\partial \theta}+\delta \sin \theta\right)\left(\delta \frac{\partial}{\partial \zeta}-\frac{R_{0}}{r} \frac{\partial}{\partial \theta}-\cos \theta \frac{\partial}{\partial \theta}+\sin \theta\right)= \\
& \left(\frac{\partial}{\partial \zeta}+\frac{1}{q} \frac{\partial}{\partial \theta}\right)\left(\delta \frac{\partial}{\partial \zeta}-\frac{R_{0}}{r} \frac{\partial}{\partial \theta}\right)+\left(\frac{\partial}{\partial \zeta}+\frac{1}{\partial \partial \theta}\right)\left(\sin \theta-\cos \theta \frac{\partial}{\partial \theta}\right)+ \\
& \left(\delta \cos \theta \frac{\partial}{\partial \theta}+\delta \sin \theta\right)\left(\delta \frac{\partial}{\partial \zeta}-\frac{R_{0}}{r} \frac{\partial}{\partial \theta}\right)+\left(\delta \cos \theta \frac{\partial}{\partial \theta}+\delta \sin \theta\right)\left(\sin \theta-\cos \theta \frac{\partial}{\partial \theta}\right) \\
& =\left(\frac{\partial}{\partial \zeta}+\frac{1}{q} \frac{\partial}{\partial \theta}\right)\left(\delta \frac{\partial}{\partial \zeta}-\frac{R_{0}}{r} \frac{\partial}{\partial \theta}\right)+\left(\sin \theta-\cos \theta \frac{\partial}{\partial \theta}\right) \frac{\partial}{\partial \zeta}+\frac{1}{\partial} \frac{\partial}{\partial \theta}\left(\sin \theta-\cos \theta \frac{\partial}{\partial \theta}\right)+ \\
& \delta \sin \theta\left(\delta \frac{\partial}{\partial \zeta}-\frac{P_{0}}{r} \frac{\partial}{\partial \theta}\right)+\delta \cos \theta \frac{\partial}{\partial \theta}\left(\delta \frac{\partial}{\partial \zeta}-\frac{P_{0}}{r} \frac{\partial}{\partial \theta}\right)= \\
& \left(\frac{\partial}{\partial \zeta}+\frac{1}{q} \frac{\partial}{\partial \theta}\right)\left(\delta \frac{\partial}{\partial \zeta}-\frac{R_{0}}{r}\right)+\left(\sin \theta-\cos \theta \frac{\partial}{\partial \theta}\right) \frac{\partial}{\partial \zeta}+\frac{1}{q}\left(\cos \theta+2 \sin \theta \frac{\partial}{\partial \theta}-\cos \theta \frac{\partial^{2}}{\partial \theta^{2}}\right)+ \\
& \left(\delta^{2} \sin \theta \frac{\partial}{\partial \zeta}-\frac{1}{q} \sin \theta \frac{\partial}{\partial \theta}\right)+\left(\delta^{2} \cos \theta \frac{\partial}{\partial \theta} \frac{\partial}{\partial \zeta}-\cos \theta \frac{1}{\partial} \frac{\partial^{2}}{\partial \theta^{2}}\right)=\left(\frac{\partial}{\partial \zeta}+\frac{1}{q} \frac{\partial}{\partial \theta}\right)\left(\delta \frac{\partial}{\partial \zeta}-\frac{R_{0}}{r}\right)+ \\
& \sin \theta\left(1+\delta^{2}\right) \frac{\partial}{\partial \zeta} \cdot \cdots\left(1-\delta^{2}\right) \cos \theta \frac{\partial}{\partial \theta} \frac{\partial}{\partial \zeta}+\frac{1}{q}\left(\cos \theta+\sin \theta \frac{\partial}{\partial \theta}-2 \cos \theta \frac{\partial^{2}}{\partial \partial^{2}}\right)
\end{aligned}
$$

com

$$
-\frac{\partial}{\partial r} \frac{1}{r} \frac{\partial}{\partial r}+\frac{1}{r} \frac{\partial^{2}}{\partial r^{2}}=\frac{1}{r^{2}} \frac{\partial}{\partial r}
$$


en

$$
\begin{aligned}
& \left(-\frac{\frac{r}{R_{0}} \cos \theta}{\sqrt{1+\delta^{2}}}\left\{\frac{\partial}{\partial r} \frac{1}{r} \frac{\partial}{\partial r}\right\}+\frac{\cos \theta}{R_{0} \sqrt{1+\delta^{2}}} \frac{\partial^{2}}{\partial r^{2}}\right) \frac{r}{\sqrt{1+\delta^{2}}} \\
= & \left(\frac{\frac{r}{R_{0}} \cos \theta}{\sqrt{1+\delta^{2}}} \frac{1}{r^{2}} \frac{\partial}{\partial r}\right) \frac{r}{\sqrt{1+\delta^{2}}}
\end{aligned}
$$

e também fazendo,

$$
\frac{1}{r} \frac{\partial}{\partial r} r \frac{\partial}{\partial r} r=\frac{\partial}{\partial r}+\frac{\partial}{\partial r}+2 \frac{\partial}{\partial r}+\frac{1}{r}
$$

$\mathrm{m}$

$$
\begin{aligned}
& \frac{\delta}{r \sqrt{1+\delta^{2}}}\left\{\frac{\partial}{\partial r} r \frac{\partial}{\partial r}+\frac{r}{R_{0}} \frac{\cos \theta}{r} \frac{\partial}{\partial r} r \frac{\partial}{\partial r} r-\frac{r}{R_{0}} \cos \theta \frac{\partial}{\partial r} r \frac{\partial}{\partial r}-\frac{r}{R_{0}} \cos \theta \frac{\partial}{\partial r}\right\} \frac{\delta}{\sqrt{1+\delta^{2}}} \\
= & \frac{\delta}{r \sqrt{1+\delta^{2}}}\left\{\frac{\partial}{\partial r} r \frac{\partial}{\partial r}+\frac{r}{R_{0}} \cos \theta\left(2 \frac{\partial}{\partial r}+\frac{1}{r}\right)-\frac{r}{R_{0}} \cos \theta \frac{\partial}{\partial r}\right\} \frac{\delta}{\sqrt{1+\delta^{2}}} \\
= & \frac{\delta}{r \sqrt{1+\delta^{2}}}\left\{\frac{\partial}{\partial r} r \frac{\partial}{\partial r}+\frac{r}{R_{0}} \cos \theta\left(\frac{\partial}{\partial r}+\frac{1}{r}\right)\right\} \frac{\delta}{\sqrt{1+\delta^{2}}}= \\
= & \frac{\delta}{r \sqrt{1+\delta^{2}}} \frac{\partial}{\partial r} r \frac{\partial}{\partial r} \frac{\delta}{\sqrt{1+\delta^{2}}}+\frac{\delta \cos \theta}{r \sqrt{1+\delta^{2}}} \frac{r^{2}}{R_{0}} \frac{\partial}{\partial r} \frac{\delta}{\sqrt{1+\delta^{2}}}+\frac{\delta^{2}}{r^{2}\left(1+\delta^{2}\right)} \frac{r}{R_{0}} \cos \theta
\end{aligned}
$$

obtém-se, entăo,

$$
\begin{aligned}
& (\vec{\nabla} \times \vec{\nabla} \times \vec{E})_{1}= \\
& \frac{1}{\sqrt{1+\delta^{2}}}\left[\frac{1}{r \partial r} \frac{\partial}{\partial \theta}-\frac{1-\frac{r}{R_{0}} \cos \theta}{r^{2}} \frac{\partial}{\partial \theta}-\frac{\delta\left(1-\frac{*}{f_{0}} \cos \theta\right)}{R_{0}} \frac{\partial}{\partial r} \frac{\partial}{\partial \zeta}-\frac{\delta\left(1-2 \frac{r}{R_{\theta}} \cos \theta\right)}{r R_{0}} \frac{\partial}{\partial \zeta}\right] E_{r} \\
& -\left[\frac{1-2 \frac{r}{R_{0}} \cos \theta}{R_{0}\left(1+\delta^{2}\right)}\left\{\left(\frac{\partial}{\partial \zeta}+\frac{1}{q \partial \theta}\right)^{2}+2 \partial \cos \partial \frac{\partial}{\partial \theta}\left(\frac{\partial}{\partial \zeta}+\frac{1}{\partial \theta}\right)-\frac{\partial}{\partial \theta}\left(\delta \sin \theta \frac{\partial}{\partial \theta}+\delta \cos \theta\right)\right]\right. \\
& +\left(\frac{1}{\sqrt{1+b^{2}}} \frac{\partial}{\partial r} \frac{1}{\partial r}+\frac{\partial}{\sqrt{1+\delta^{2}}} \frac{\frac{r}{r^{2}}}{\partial r} \frac{\partial}{\partial r}\right) \frac{r}{\sqrt{1+\delta^{2}}}-\frac{\delta \cos \theta}{r \sqrt{1+\delta^{2}}} \frac{r}{h_{0}} \frac{\partial}{\partial r \sqrt{1+\delta^{2}}} \\
& \left.+\frac{\delta}{r \sqrt{1+\delta^{2}}} \frac{\partial}{\partial r} r \frac{\partial}{\partial r} \frac{\delta}{\sqrt{1+\delta^{2}}}+2 \frac{\delta \cos \theta}{r \sqrt{1+\delta^{2}}} \frac{r}{R_{0}} \frac{\partial}{\partial r} \frac{\delta}{\sqrt{1+\delta^{2}}}+\frac{\delta^{2}}{r^{2}\left(1+\delta^{2}\right)} \frac{r}{R_{0}} \cos \theta\right] E_{\perp} \\
& +\left[\frac { 1 - 2 \frac { r } { R _ { 0 } } \operatorname { c o s } \theta } { R _ { 0 } ^ { 2 } ( 1 + \delta ^ { 2 } ) } \left\{\left(\frac{\partial}{\partial \zeta}+\frac{1}{q \partial \theta}\right)\left(\delta \frac{\partial}{\partial \zeta}-\frac{R_{0}}{r}\right)+\sin \theta\left(1+\delta^{2}\right) \frac{\partial}{\partial \zeta}-\left(1-\delta^{2}\right) \cos \theta \frac{\partial}{\partial \phi} \frac{\partial}{\partial \zeta}+\right.\right. \\
& \left.\frac{1}{q}\left(\cos \theta+\sin \theta \frac{\partial}{\partial \theta}-2 \cos \theta \frac{\partial^{2}}{\partial \theta^{2}}\right)\right\}+\left\{\frac{1}{\sqrt{1+\delta^{2}}} \frac{\partial}{\partial r} \frac{1}{r} \frac{\partial}{\partial *}+\frac{\frac{5}{\Omega_{0}} \cos \theta}{\sqrt{1+\delta^{2}}} \frac{1}{r^{2}} \frac{\partial}{\partial r}\right\} \frac{r b}{\sqrt{1+\delta^{2}}} \\
& \left.-\frac{\delta}{r \sqrt{1+\delta^{2}}} \frac{\partial}{\partial r} r \frac{\partial}{\partial r} \frac{1}{\sqrt{1+\delta^{2}}}-\frac{\delta \cos \theta}{r \sqrt{1+\delta^{2}}} \frac{r}{R_{0}} \frac{\partial}{\partial r} \frac{1}{\sqrt{1+\delta^{2}}}-\frac{\delta}{r^{2}\left(1+\delta^{2}\right)} \frac{r}{R_{0}} \cos \theta\right] E_{1}(196)
\end{aligned}
$$


$(\vec{\nabla} \times \ddot{\nabla} \times \vec{E})_{\perp}=$

$\frac{1}{\sqrt{1+\delta^{2}}}\left[\frac{1}{r} \frac{\partial}{\partial r} \frac{\partial}{\partial \theta}-\frac{1-\frac{r}{R_{0}} \cos \theta}{r^{2}} \frac{\partial}{\partial \theta}-\frac{\delta\left(1-\frac{r}{R_{0}} \cos \theta\right)}{R_{0}} \frac{\partial}{\partial r} \frac{\partial}{\partial \zeta}-\frac{\delta\left(1-2 \frac{r}{R_{b}} \cos \theta\right)}{r R_{0}} \frac{\partial}{\partial \zeta}\right] E_{r}$

$-\left[\frac{\left(\frac{\partial}{\partial \zeta}+\frac{1}{q} \frac{\partial}{\partial \theta}\right)^{2}}{R_{0}^{2}\left(1+\delta^{2}\right)}+\frac{1}{\sqrt{1+\delta^{2}}} \frac{\partial}{\partial r} \frac{1}{r} \frac{\partial}{\partial r} \frac{r}{\sqrt{1+\delta^{2}}}+\frac{\delta}{r \sqrt{1+\delta^{2}}} \frac{\partial}{\partial r} r \frac{\partial}{\partial r} \frac{\delta}{\sqrt{1+\delta^{2}}}\right.$

$\frac{2 \frac{r}{R_{0}} \cos \theta\left(\frac{\partial}{\partial \zeta}+\frac{1}{q} \partial\right)^{2}}{R_{0}^{2}\left(1+\delta^{2}\right)}+\frac{2 \delta \cos \theta \frac{\partial}{\partial \theta}\left(\frac{\partial}{\partial \zeta}+\frac{1}{a} \frac{\partial}{\partial \theta}\right)}{R_{0}^{2}\left(1+\delta^{2}\right)}-\frac{\delta \sin \theta \frac{\partial}{\partial \theta}}{q R_{0}^{2}\left(1+\delta^{2}\right)}-\frac{\delta \cos \theta}{q R_{0}^{2}\left(1+\delta^{2}\right)}$

$\left.+\frac{\frac{r}{R_{0}} \cos \theta}{\sqrt{1+\delta^{2}}} \frac{1}{r^{2}} \frac{\partial}{\partial r} \frac{r}{\sqrt{1+\delta^{2}}}+\frac{\frac{r}{\phi_{0}} \delta \cos \theta}{r \sqrt{1+\delta^{2}}} \frac{\partial}{\partial r} \frac{\delta}{\sqrt{1+\delta^{2}}}+\frac{\frac{r}{\delta_{0}} \delta^{2}}{r^{2}\left(1+\delta^{2}\right)} \cos \theta\right] E_{\perp}$

$+\left[\frac{\left(\frac{\partial}{\partial \zeta}+\frac{1}{q} \frac{\partial}{\partial \theta}\right)\left(\delta \frac{\partial}{\partial \zeta}-\frac{R_{0}}{r}\right)}{R_{0}^{2}\left(1+\delta^{2}\right)}-\frac{\delta}{r \sqrt{1+\delta^{2}}} \frac{\partial}{\partial r} r \frac{\partial}{\partial r} \frac{1}{\sqrt{1+\delta^{2}}}+\frac{1}{\sqrt{1+\delta^{2}}} \frac{\partial}{\partial r} \frac{1}{r} \frac{\partial}{\partial r} \frac{r \delta}{\sqrt{1+\delta^{2}}}\right.$

$-\frac{2 \frac{r}{R_{0}} \cos \theta\left(\frac{\partial}{\partial \zeta}+\frac{1}{q} \frac{\partial}{\partial j}\right)\left(\delta \frac{\partial}{\partial \zeta}-\frac{R_{0}}{r}\right)}{R_{0}^{2}\left(1+\delta^{2}\right)}+\frac{\frac{r}{R_{0}} \cos \theta}{\sqrt{1+\delta^{2}}} \frac{1}{r^{2}} \frac{\partial}{\partial r} \frac{r \delta}{\sqrt{1+\delta^{2}}}$

$+\frac{1}{R_{0}^{2}\left(1+\delta^{2}\right)}\left\{\sin \theta\left(1+\delta^{2}\right) \frac{\partial}{\partial \zeta}-\left(1-\delta^{2}\right) \cos \theta \frac{\partial}{\partial \theta} \frac{\partial}{\partial \zeta}\right.$

$\left.\left.+\frac{1}{q}\left(\sin \theta \frac{\partial}{\partial \theta}-2 \cos \theta \frac{\partial^{2}}{\partial \theta^{2}}\right)\right\}-\frac{\frac{r}{r_{0}} \delta \cos \theta}{r \sqrt{1+\delta^{2}}} \frac{\partial}{\partial r} \frac{1}{\sqrt{1+\delta^{2}}}\right] E_{\|}$

$(\vec{\nabla} \times \vec{\nabla} \times \vec{E})_{1}=$

$\frac{1}{\sqrt{1+\partial^{2}}}\left[\frac{1}{r} \frac{\partial}{\partial r} \frac{\partial}{\partial \theta}-\frac{1-\frac{r}{R_{0}} \cos \theta}{r^{2}} \frac{\partial}{\partial \theta}-\frac{\delta\left(1-\frac{r}{R_{0}} \cos \theta\right)}{R_{0}} \frac{\partial}{\partial r} \frac{\partial}{\partial \zeta}-\frac{\delta\left(1-2 \frac{r}{R_{0}} \cos \theta\right)}{r R_{0}} \frac{\partial}{\partial \zeta}\right] E_{r}$

$-\left[\frac{\left(\frac{\partial}{\partial \zeta}+\frac{1}{\partial \theta}\right)^{2}}{R_{0}^{2}\left(1+\delta^{2}\right)}+\frac{1}{\sqrt{1+\delta^{2}}} \frac{\partial}{\partial r} \frac{1}{r} \frac{\partial}{\partial r} \frac{r}{\sqrt{1+\delta^{2}}}+\frac{\delta}{r \sqrt{1+\delta^{2}}} \frac{\partial}{\partial r} r \frac{\partial}{\partial r} \frac{\delta}{\sqrt{1+\delta^{2}}}-\frac{2 \frac{r}{R_{0}} \cos \theta \frac{\partial^{2}}{R_{0}^{2}}}{R_{0}^{2}\left(1+\delta^{2}\right)}\right.$

$\left.+\frac{2 \delta_{\delta_{1}} \cos \theta}{r R_{0}\left(1+\delta^{2}\right)} \frac{\partial}{\partial \theta} r-\frac{\delta \sin \theta \frac{\partial}{\partial \phi}}{q R_{0}^{2}\left(1+\delta^{2}\right)}+\frac{\frac{r}{R_{0}} \cos \theta}{\sqrt{1+\delta^{2}}} \frac{1}{r^{2}} \frac{\partial}{\partial r} \frac{r}{\sqrt{1+\delta^{2}}}+\frac{\frac{r}{R_{0}} \delta \cos \theta}{r \sqrt{l+\delta^{2}}} \frac{\partial}{\partial r} \frac{\delta}{\sqrt{1+\delta^{2}}}\right] E_{\perp}$

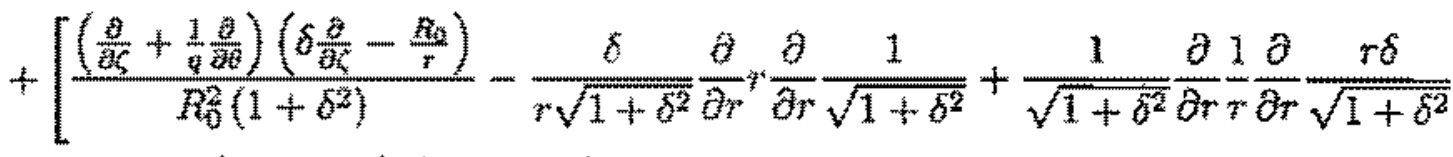

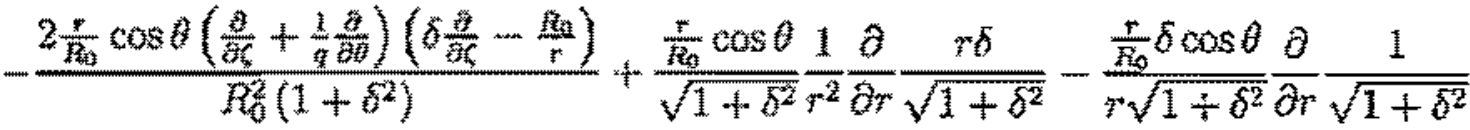

$\left.+\frac{1}{R_{0}^{2}\left(1+\delta^{2}\right)}\left\{\sin \theta\left(1+\delta^{2}\right) \frac{\partial}{\partial \zeta}-\left(1-\delta^{2}\right) \cos \theta \frac{\partial}{\partial \theta} \frac{\partial}{\partial \zeta} \div \frac{1}{q}\left(\sin \theta \frac{\partial}{\partial \theta}-2 \cos \theta \frac{\partial^{2}}{\partial \theta^{2}}\right)\right\}\right] E_{\|}$ 


$$
\begin{aligned}
& (\vec{\nabla} \times \vec{\nabla} \times \vec{E})_{\perp}= \\
& \frac{1}{\sqrt{1+\delta^{2}}}\left[\frac{1}{r} \frac{\partial}{\partial \theta} \frac{\partial}{\partial r}-\frac{1}{r^{2}} \frac{\partial}{\partial \theta}-\frac{\delta}{R_{0}} \frac{\partial}{\partial \zeta} \frac{\partial}{\partial r}-\frac{\delta}{r R_{0}} \frac{\partial}{\partial \zeta}\right] E_{r}+ \\
& \frac{2 \frac{r}{R_{0}} \cos \theta}{\sqrt{1+\delta^{2}}}\left[\frac{1}{2 r^{2}} \frac{\partial}{\partial \theta}+\frac{\delta}{2 R_{\theta}} \frac{\partial}{\partial r} \frac{\partial}{\partial \zeta}+\frac{\delta}{r R_{0}} \frac{\partial}{\partial \zeta}\right] E_{r} \\
& -\left[\frac{\left(\frac{\partial}{\partial}+\frac{1}{\partial} \frac{\partial}{\partial 2}\right)^{2}}{R_{0}^{2}\left(1+\frac{\left.\delta^{2}\right)}{2}\right.}+\frac{1}{\sqrt{1+\delta^{2}}} \frac{\partial}{\partial r} \frac{1}{\partial r} \frac{\gamma}{\sqrt{1+\delta^{2}}}+\frac{\delta}{r \sqrt{1+\delta^{2}} \frac{\partial}{\partial r}}+\frac{\partial}{\partial r} \frac{\delta}{\sqrt{1+\delta^{2}}}\right] E_{1} \\
& +2 \frac{r}{R_{0}} \sin \theta\left[\frac{\delta^{2} \frac{\partial}{\partial \theta}}{2 r^{2}\left(1+\delta^{2}\right)}\right] E_{1} \\
& -2 \frac{r}{R_{0}} \cos \theta\left[-\frac{\frac{\partial^{2}}{\partial K^{2}}}{R_{0}^{2}\left(1+\delta^{2}\right)}+\frac{\delta}{R_{R}\left(1+\delta^{2}\right)} \frac{\partial}{\partial \theta}{ }^{*}\right. \\
& \left.+\frac{1}{2 \sqrt{1+\delta^{2}}} \frac{1}{r^{2}} \frac{\partial}{\partial r} \frac{r}{\sqrt{1+\delta^{2}}}+\frac{\delta}{2 r \sqrt{1+\delta^{2}}} \frac{\partial}{\partial r} \frac{\delta}{\sqrt{1+\delta^{2}}}\right] E_{\perp} \\
& +\left[\frac{\left(\frac{\partial}{\partial \zeta}+\frac{1}{q} \frac{\partial}{\partial g}\right)\left(\delta \frac{\partial}{\partial \zeta}-\frac{n_{a}}{r}\right)}{R_{0}^{2}\left(1+\delta^{2}\right)}-\frac{\delta}{r \sqrt{1+\delta^{2}}} \frac{\partial}{\partial r} r \frac{\partial}{\partial r} \frac{1}{\sqrt{1+\delta^{2}}}+\frac{1}{\sqrt{1+\delta^{2}}} \frac{\partial}{\partial r} \frac{1}{r} \frac{\partial}{\partial r} \frac{r \delta}{\sqrt{1+\delta^{2}}}\right] E_{\|} \\
& +2 \frac{r}{R_{0}} \cos \theta\left[\frac{\frac{1}{r^{2}}}{\sqrt{1+\delta^{2}}} \frac{\partial}{\partial r} \frac{r \delta}{\sqrt{1+\delta^{2}}}-\frac{\delta}{2 r \sqrt{1+\delta^{2}}} \frac{\partial}{\partial r} \frac{1}{\sqrt{1+\delta^{2}}}-\frac{\left(\frac{\partial}{\partial \zeta}+\frac{2}{q} \frac{\partial}{\partial \theta}\right)\left(\delta \frac{\partial}{\partial \zeta}-\frac{R_{0}}{r}\right)}{R_{\phi}^{2}\left(1+\delta^{2}\right)}+\right. \\
& \left.-\frac{1}{r R_{0}\left(1+\delta^{2}\right)}\left\{\frac{\left(1-\delta^{2}\right)}{2} \frac{\partial}{\partial \theta} \frac{\partial}{\partial \zeta}+\frac{1}{q} \frac{\partial^{2}}{\partial \theta^{2}}\right\}\right] E_{\|} \\
& +2 \frac{r}{R_{0}} \sin \theta\left[\frac{1}{2 r R_{0}}\left(\frac{\partial}{\partial \zeta}+\frac{1}{q\left(1+\delta^{2}\right)} \frac{\partial}{\partial \theta}\right)\right] E_{\mathrm{l}}
\end{aligned}
$$


Eliminando $E_{\|}$através de $\vec{\nabla} \cdot \vec{J}=0$

$$
\vec{\nabla} \cdot \vec{J}=\frac{1}{r R} \frac{\partial}{\partial r} r R J_{r}+\frac{1}{r R} \frac{\partial}{\partial \theta} R J_{\theta}+\frac{1}{R} \frac{\partial}{\partial \zeta} J_{z}=0 .
$$

Desprezando a toroidicidade na equação acima,

$$
R=R_{0}
$$

chega-se a

$$
\frac{1}{r} \frac{\partial}{\partial r} r J_{r}+\frac{1}{r} \frac{\partial}{\partial \theta} J_{\theta}+\frac{1}{R_{0}} \frac{\partial}{\partial \zeta} J_{z}=0
$$

ou

$$
\frac{1}{r} \frac{\partial}{\partial r} r J_{r}+i k_{\theta} J_{\theta}+i k_{z} J_{z}=0
$$

Substituindo as transformaçōes do início deste apêndice, chega-se a

$$
\begin{aligned}
& \frac{1}{r} \frac{\partial}{\partial r} r J_{r}+i\left(k_{\perp}+\delta k_{\|}\right) / \sqrt{1+\delta^{2}} J_{\theta}+i\left(k_{\|}-\delta k_{\perp}\right) / \sqrt{1+\delta^{2}} J_{z}=0 \\
& \frac{1}{r} \frac{\partial}{\partial r} r J_{\tau}+i \frac{\left(k_{\perp}+\delta k_{\|}\right)}{\sqrt{1+\delta^{2}}} \frac{\left(J_{\perp}+\delta J_{\|}\right)}{\sqrt{1+\delta^{2}}}+i \frac{\left(k_{\|}-\delta k_{\perp}\right)}{\sqrt{1+\delta^{2}}} \frac{\left(J_{\|}-\delta J_{\perp}\right)}{\sqrt{1+\delta^{2}}}=0
\end{aligned}
$$

ou, simplificando,

$$
i\left(k_{\|} J_{\|}+k_{\perp} J_{\perp}\right)+\frac{1}{r} \frac{\partial}{\partial r} r J_{r}=0 .
$$

\section{E.2.3 Eliminando $E_{\|}$}

De

$$
\vec{J}=\frac{1}{i \mu_{0} \omega} \frac{\omega^{2}}{c^{2}} \overleftrightarrow{\chi} \cdot \vec{E}
$$

ou 


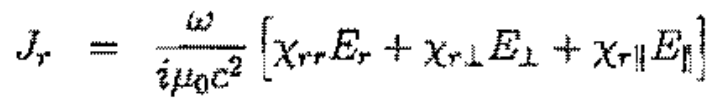

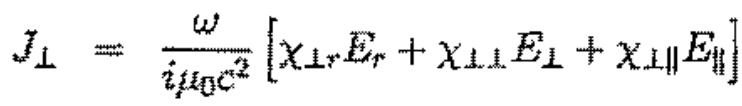

$$
\begin{aligned}
& I_{n}=\frac{\omega}{i \mu_{0} c^{2}}\left[\chi_{r} E_{r}+\chi_{\| \perp} E_{\perp}+\chi_{\|\|} E_{\|}\right]
\end{aligned}
$$

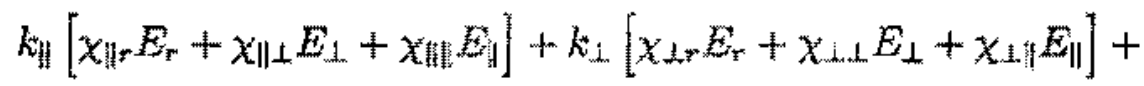

$$
\begin{aligned}
& =\frac{1}{r} \frac{\partial}{\partial r}\left(i r\left[\chi_{r r} E_{r}+\chi_{r_{1}} E_{1}+\chi_{r n} E_{\| 1}\right]\right)
\end{aligned}
$$

Com esta última expressão, pode-se eliminar a componente paralela do campo elétrico das expressões de $(\vec{\nabla} \times \vec{\nabla} \times \vec{E})$, reduzindo então o sistema a ser resolvido pelo coligo.

Abaixo está listada uma versão mais simples das componentes do tensor dieletrico. Usando seus elementos e mais as Eqs.(205), e mitroduzindo as expressōes desenvolvidas neste apendice para as componentes perpendicular e radial de $(\vec{\nabla} \times \vec{\nabla} \times \vec{E})$, devidamente

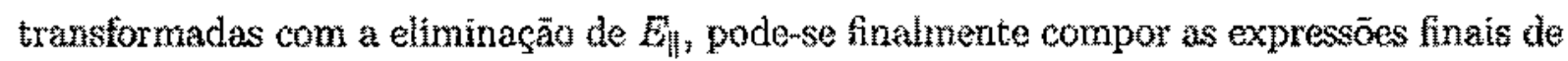
da Eq (184), com base nas quais o código foi criado. 


$$
\begin{aligned}
\frac{\omega^{2}}{c^{2}} \chi_{r r} & =\frac{\omega^{2}}{v_{A}^{2}} \alpha_{i}+2 \hat{\epsilon} \cos \theta \frac{\omega^{2}}{v_{A}^{2}} \alpha_{i} \\
\frac{\omega^{2}}{c^{2}} \chi_{r \perp} & =-i \frac{\omega}{\omega_{c i}} \frac{\omega^{2}}{v_{A}^{2}} \alpha_{i}+3 i \cos \theta \frac{\omega}{\omega_{c i}} \frac{\omega^{2}}{v_{A}^{2}} \alpha_{i}-i k_{\|} \frac{(r \delta) \prime}{r\left(1+\delta^{2}\right)} \\
\frac{\omega^{2}}{c^{2}} \chi_{\perp r} & =i \frac{\omega}{\omega_{c i}} \frac{\omega^{2}}{v_{A}^{2}} \alpha_{i}-3 i \cos \theta \frac{\omega}{\omega_{c i}} \frac{\omega^{2}}{v_{A}^{2}} \alpha_{i}+i k_{\|} \frac{(r \delta)^{\prime}}{r\left(1+\delta^{2}\right)} \\
\frac{\omega^{2}}{c^{2}} \chi_{\perp \perp} & =\frac{\omega^{2}}{v_{A}^{2}} \alpha_{i}+2 \hat{\epsilon} \cos \theta \frac{\omega^{2}}{v_{A}^{2}} \alpha_{i} \\
\frac{\omega^{2}}{c^{2}} \chi_{r \|} & =i k_{\perp} \frac{\omega^{2}}{v_{A}^{2}} \frac{\omega_{c i}}{\omega\left|k_{\|}\right|}\left[1+\rho_{e} Z\left(\rho_{e}\right)\right] \\
\frac{\omega^{2}}{c^{2}} \chi_{\| r} & =-\frac{\omega^{2}}{c^{2}} \chi_{r \|} \\
\frac{\omega^{2}}{c^{2}} \chi_{\perp \|} & =-\frac{d}{d r} \frac{\omega^{2}}{v_{A}^{2}} \frac{\omega_{c i}}{\omega\left|k_{\|}\right|}\left[1+\rho_{e} Z\left(\rho_{e}\right)\right] \\
\frac{\omega^{2}}{c^{2}} \chi_{\| \perp} & =\frac{\omega^{2}}{v_{A}^{2}} \frac{\omega_{c i}}{\omega\left|k_{\|}\right|}\left[1+\rho_{c} Z\left(\rho_{c}\right)\right] \frac{1}{r} \frac{d}{d r} r \\
\frac{\omega^{2}}{c^{2}} \chi_{\|\|} & =\frac{\omega^{2}}{v_{A}^{2}} \frac{1+\rho Z(\rho)}{k_{\|}^{2} \rho_{s}^{2}\left[1+\frac{i \nu}{\left|k_{\|}\right| v_{T e}} Z(\rho)\right]}
\end{aligned}
$$

onde

$$
\rho_{e}=\frac{\omega}{\left|k_{\|}\right| v_{T e}}, \quad \quad \rho=\frac{\omega+i \nu}{\left|k_{\|}\right| v_{T e}}, \quad \quad \nu \propto \frac{n_{e}}{T_{e}^{3 / 2}}
$$




\section{F Forças ponderomotoras}

Começando pela equação da continuidade,

$$
\begin{aligned}
\frac{\partial}{\partial t} n_{\alpha}+\vec{\nabla} \cdot\left(n_{\alpha} \vec{v}_{\alpha}\right) & =0 \\
\frac{\partial}{\partial t}\left(m_{\alpha} n_{\alpha}\right)+\vec{\nabla} \cdot\left(m_{\alpha} n_{\alpha \alpha} \vec{v}_{\alpha}\right) & =0 \\
\vec{v}_{\alpha} \frac{\partial}{\partial t}\left(m_{\alpha \alpha} n_{\alpha}\right)+\vec{v}_{\alpha} \vec{\nabla} \cdot\left(m_{\alpha \alpha} n_{\alpha} \vec{v}_{\alpha}\right) & =0 \\
\frac{\partial}{\partial t}\left(m_{\alpha \alpha} n_{\alpha} \vec{v}_{\alpha}\right)-m_{\alpha} n \frac{\partial}{\partial t} \frac{\partial}{\partial t}+\vec{\nabla} \cdot\left(m_{\alpha \alpha} n_{\alpha} \vec{v}_{\alpha} \vec{v}_{\alpha}\right)-m_{\alpha} n_{\alpha}\left(\vec{v}_{\alpha} \cdot \vec{\nabla}\right) \vec{v}_{\alpha \alpha} & =0
\end{aligned}
$$

com a equação de momento

$$
\begin{aligned}
& m_{\alpha} n_{\alpha} \frac{\overrightarrow{d v_{\alpha}}}{d t}=-\vec{\nabla} P_{\alpha}-\vec{\nabla} \Pi_{\alpha}+Z_{\alpha} n_{\alpha} e_{\alpha}\left(\ddot{E}+\frac{1}{c}\left[\vec{v}_{\alpha} \times \vec{B}\right]\right)+R_{\alpha \alpha}
\end{aligned}
$$

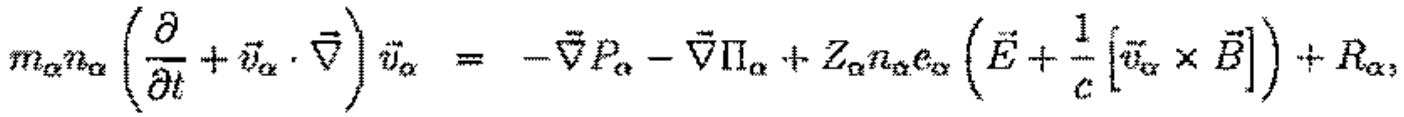

$$
\begin{aligned}
& \frac{\partial}{\partial t}\left(m_{\alpha \alpha} n_{t \alpha} \vec{v}_{\alpha}\right)+\vec{\nabla} \cdot\left(m_{\alpha} n_{\alpha \alpha} \vec{v}_{\alpha} \vec{v}_{\alpha}\right)=-\vec{\nabla} P_{\alpha}-\vec{\nabla} n_{\alpha z}+z_{\alpha} n_{\alpha} e_{\alpha}\left(\vec{E}+\frac{1}{c}\left[\vec{v}_{\alpha} \times \vec{\theta}\right]\right)+R_{\alpha}
\end{aligned}
$$

Supondo pressä̀ isotrópica, $\ddot{\nabla} P_{\alpha}=0, Z_{\alpha}=1$, e $R_{\alpha x}=0$,

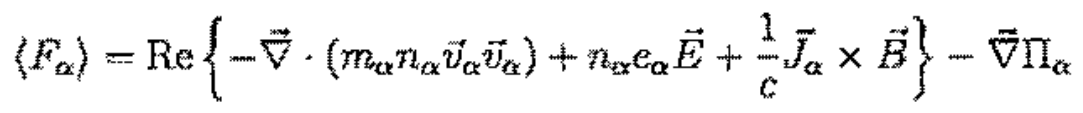

$$
\begin{aligned}
m_{\alpha} n_{\alpha} \vec{v}_{\alpha} \vec{v}_{\alpha} & \equiv \frac{m_{\alpha \alpha}}{n_{\alpha \alpha} e_{\alpha \alpha}^{2}} \vec{J}_{\alpha}=\frac{1}{\epsilon_{0} \omega_{p \alpha}^{2}} \overrightarrow{J_{\alpha}} \vec{J}_{\alpha} \\
\frac{1}{c} \vec{J}_{\alpha} \times \vec{B} & \equiv \frac{1}{c} \overrightarrow{J_{\alpha}} \times\left(-\frac{i f_{\alpha}^{2}}{\omega} \vec{\nabla} \times \vec{E}\right)=\frac{1}{i \omega}, \overrightarrow{J_{\alpha}} \times(\vec{\nabla} \times \vec{E})
\end{aligned}
$$

$$
F^{2}=\operatorname{Re}\left\{-\ddot{\nabla} \cdot\left(\frac{1}{\epsilon_{0} \omega_{\alpha \alpha}^{2}} \vec{J}_{\alpha} \tilde{J}_{\alpha}\right)+e_{\alpha}\left[n_{\alpha} \vec{E}+\frac{1}{i u e_{\alpha}} \vec{J}_{\alpha} \times(\vec{\nabla} \times \vec{E})\right]\right\}-\vec{\nabla} \Pi_{\alpha}
$$

Da equaçäo da continuidade, itwn $_{\alpha}=\vec{\nabla} \cdot\left(\frac{1}{e_{\alpha}}\right)$, entäo

$$
F=R e\left\{-\vec{\nabla} \cdot\left(\frac{1}{\epsilon_{0} \omega_{p \alpha}^{2}} \ddot{J}_{\alpha} \overrightarrow{J_{\alpha}}\right)+\frac{1}{i \omega}\left[\vec{E} \vec{\nabla} \cdot \vec{J}_{\alpha}+\ddot{J}_{\alpha} \times(\vec{\nabla} \times \vec{E})\right]\right\}-\vec{\nabla} \Pi_{\alpha x}
$$




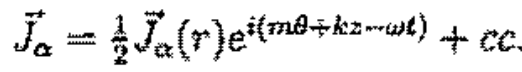

$$
\begin{aligned}
& \vec{B}=\frac{1}{2} \vec{E}(r) e^{i(m \theta+k t-\omega t)}+c c .
\end{aligned}
$$

$F_{\alpha}=\operatorname{Re}\left\{-\vec{\nabla} \cdot\left(\frac{1}{4 \epsilon_{0} \omega_{p \alpha}^{2}} \vec{J}_{\alpha}^{*} \vec{J}_{\alpha}\right)+\frac{1}{4 i \omega}\left[\ddot{E}^{*}\left(\vec{\nabla} \cdot \overrightarrow{J_{\alpha}}\right)+\vec{J}_{\alpha}^{*}(\vec{\nabla} \times \vec{E})\right]+c \alpha\right\}-\vec{\nabla} \Pi_{\alpha}$ $F_{\alpha}=$

$-\operatorname{Re}\left\{\vec{J}_{\alpha}^{*}\left(\vec{\nabla} \cdot \frac{1}{4 \epsilon_{0} \omega_{p \alpha}^{2}} \vec{J}_{\alpha}\right)+\left(\frac{1}{4 \varepsilon_{0} \omega_{p \alpha}^{2}} \vec{J}_{\alpha}^{*} \cdot \vec{\nabla}\right) \vec{J}_{\alpha}^{*}-\frac{1}{4 i_{\alpha}}\left[\vec{E}^{*}\left(\vec{\nabla} \cdot \vec{J}_{\alpha}\right)+\vec{J}_{\alpha \alpha}^{*} \times(\vec{\nabla} \times \vec{E})\right]+c_{\alpha}\right\}$ $-\vec{\nabla} \Pi_{x}$

A componente $\theta$ :

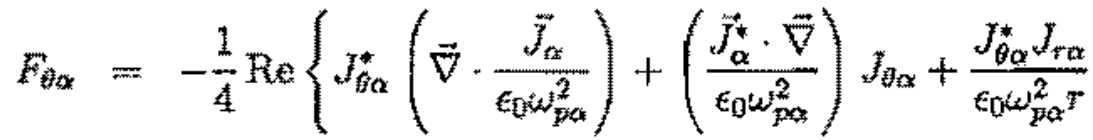

$$
\begin{aligned}
& \left.-\frac{1}{i \omega}\left[E_{0}^{*}\left(\ddot{\nabla} \cdot \overrightarrow{z_{\alpha}}\right)+J_{z \alpha}^{*}(\ddot{\nabla} \times \vec{E})_{r}-J_{r \alpha}^{*}\left(\vec{\nabla} * \overrightarrow{E^{H}}\right)_{z}\right]+c_{\alpha}\right\}-\vec{\nabla} \Pi_{\alpha}
\end{aligned}
$$

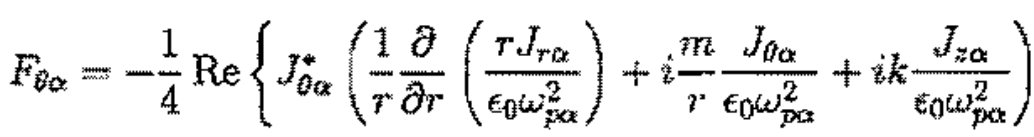

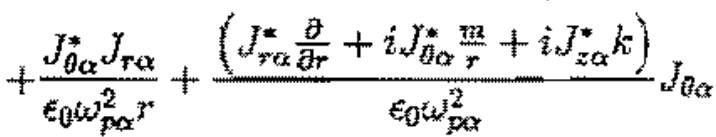

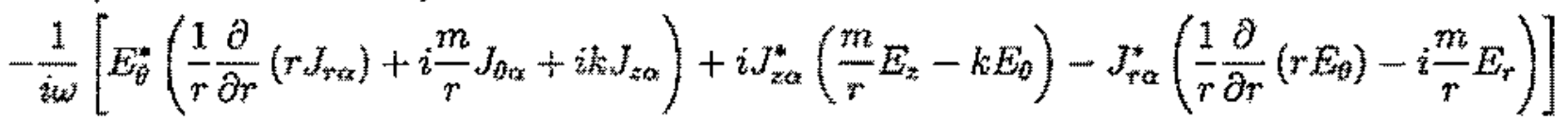

$$
\begin{aligned}
& +c c\}-\nabla \Pi_{a}
\end{aligned}
$$

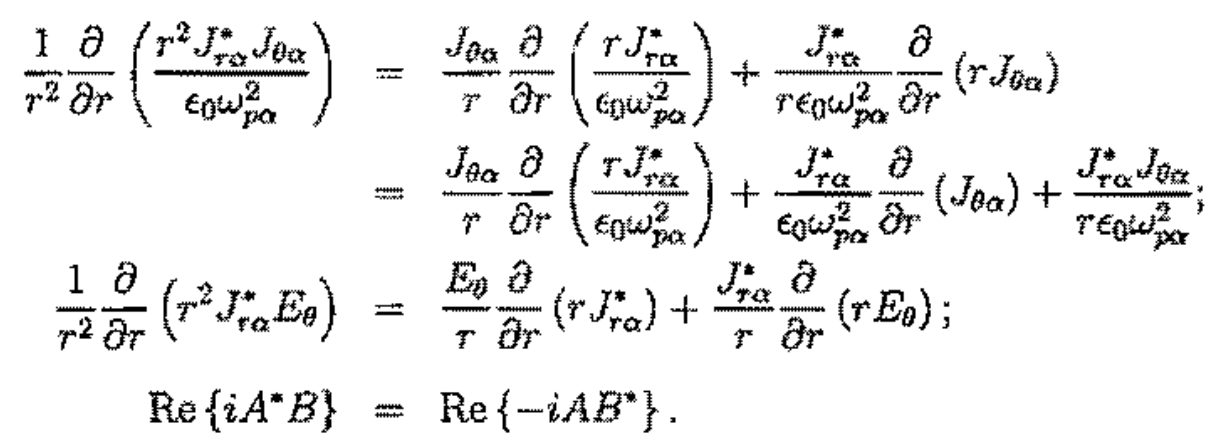




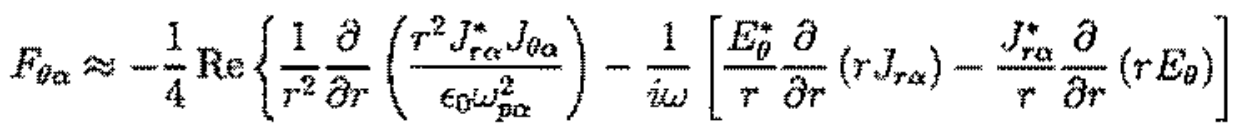

$$
\begin{aligned}
& \left.-\frac{m}{t_{t}}\left(E_{t}^{*} J_{b \alpha}+J_{r \alpha}^{*} E_{r}+J_{z \alpha}^{*} E_{z}\right)+c c\right)-\vec{\nabla} \Lambda_{\alpha}
\end{aligned}
$$

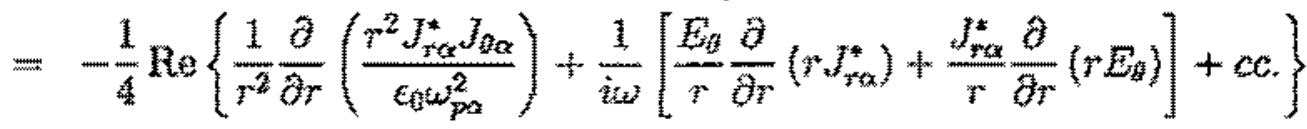

$$
\begin{aligned}
& -m \Pi_{o}+\frac{m \operatorname{Re}}{\omega t}\left(\frac{\overrightarrow{E^{*}} \cdot \overrightarrow{j_{a}^{*}}}{2}\right) \\
& =\frac{m}{\omega r} P_{\alpha}-\operatorname{Re}\left\{\frac{1}{4 r^{2}} \frac{\partial}{\partial r}\left[r^{2}\left(\frac{J_{r \alpha}^{*} y_{0 \alpha}}{\epsilon_{0} \omega_{\gamma \alpha \alpha}^{2}}+\frac{J_{r \alpha}^{*} E_{j}}{\omega_{\omega}}\right)\right]+\omega_{\alpha}\right\}-\vec{\nabla} \Pi_{\alpha} \\
& =\frac{m}{\omega r} P_{\alpha}-\operatorname{Re}\left\{\frac{1}{4 r^{2}} \frac{\partial}{\partial r}\left[r^{2} J_{r \alpha}^{*}\left(\frac{J_{0 \alpha}}{\epsilon_{0} \psi_{p \alpha \alpha}^{2}}+\frac{E_{0}}{i \omega}\right)\right]+c c_{r}\right\}-\vec{\nabla} \Pi_{\alpha}
\end{aligned}
$$




\section{F.0.4 Forças Viscosas Poloidais}

\section{Para ondas cinéticas}

Da equaçăo de conțimuìade:

$$
\begin{aligned}
& \frac{\partial}{\partial t} \tilde{n}=-\vec{\nabla} \cdot(n \dot{n}),
\end{aligned}
$$

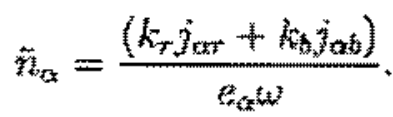

A força de giro-viscosidade:

$$
\begin{aligned}
& \frac{\partial}{\partial r^{r}}\left\langle\mathrm{~L}_{\left.\rho_{r}\right\rangle_{i}}=\frac{1}{2} m_{i} p_{i}^{2} \omega_{c i} \frac{\partial}{\partial r}\left\langle\tilde{n}_{i}\left(\frac{\partial \hat{V}_{r}^{(i)}}{\partial r^{r}}-\frac{\partial \hat{V}_{\theta}^{(i)}}{r \partial \theta}\right)\right\rangle\right.
\end{aligned}
$$

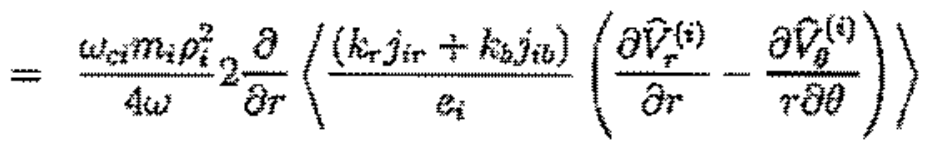

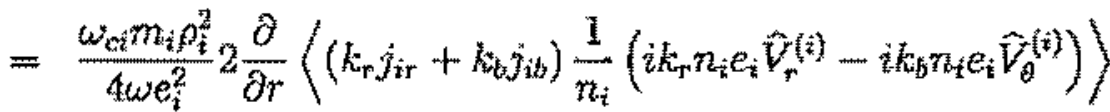

$$
\begin{aligned}
& =\frac{\omega_{a i} m_{i} p_{i}^{2}}{4 k_{2} e_{i}^{2}} 2 \frac{\partial}{\partial r}\left[\frac{1}{n_{i}}\left(k_{\mathrm{r}} j_{i \mathrm{r}}+k_{\mathrm{b}} j_{\mathrm{ib}}\right)\left(i k_{r} j_{\mathrm{ir}}-i k_{\mathrm{k}} j_{\mathrm{ib}}\right)\right]
\end{aligned}
$$

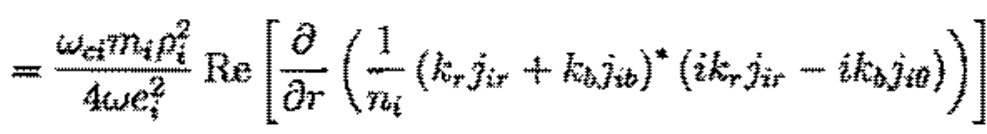

$$
\begin{aligned}
& =\frac{4 \pi \omega_{c i} \rho_{i}^{2}}{4 \omega} \operatorname{Re}\left[\frac{\partial}{\partial r}\left(\frac{m_{i}}{4 \pi n_{i} e_{i}^{2}}\left(k_{r} j_{i r}+k_{b} j_{i b}\right)^{*}\left(i k_{r} j_{i r}-i k_{b} j_{i \theta}\right)\right)\right]
\end{aligned}
$$

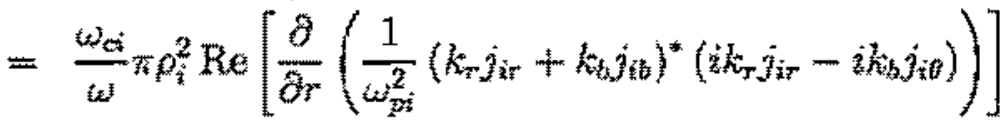

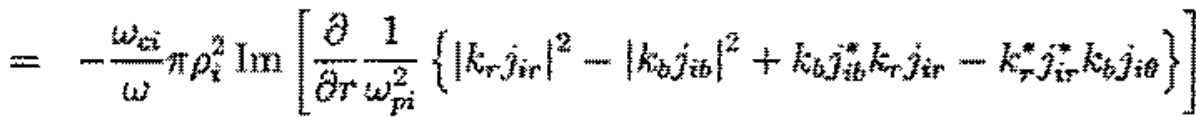

$$
\begin{aligned}
& =-\frac{\omega_{r i}}{\omega} \pi \rho_{i}^{2} \operatorname{Im}\left[\frac{\partial}{\partial r}\left\{\frac{1}{\omega_{p i}^{2}}\left(\left|k_{r} j_{i r}\right|^{2}-\left|k_{b} j_{i b}\right|^{2}+k_{b} j_{i b}^{*} k_{r} j_{i r}-k_{r} j_{i r}^{*} k_{b} j_{i \theta}\right)\right\}\right]
\end{aligned}
$$

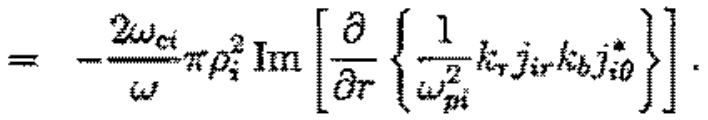

Como

$$
\begin{aligned}
& j_{i r}=-i \frac{\omega}{4 \pi}\left(\varepsilon_{12} E_{b}+\varepsilon_{11} E_{r}\right), \\
& j_{i b}=-\frac{\omega}{4 \pi}\left(\varepsilon_{21} E_{r}+\varepsilon_{22} E_{r_{b}}\right)
\end{aligned}
$$


chega-se a

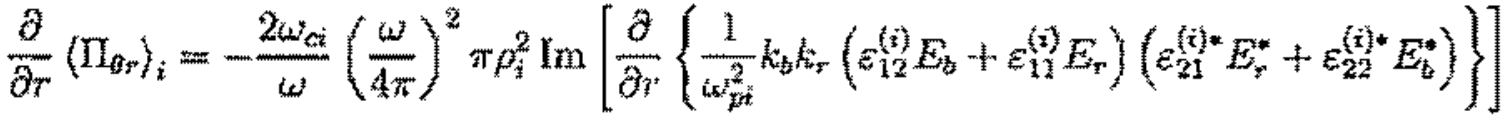

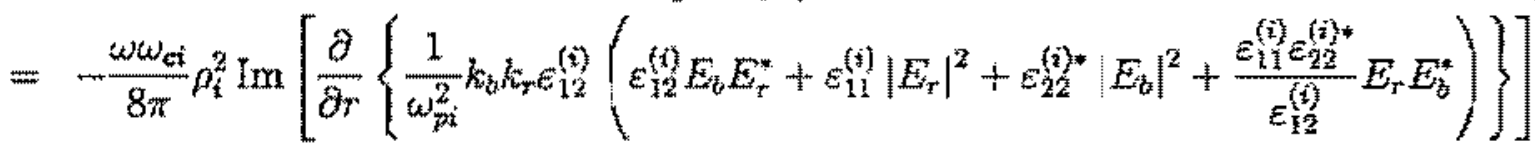

$$
\begin{aligned}
& =-\frac{\omega \omega_{c i}}{8 \pi} \rho_{i}^{2} \operatorname{Im}\left[\frac{\partial}{\partial r}\left\{\frac{k_{b} k_{r} \varepsilon_{12}^{(i)}}{\omega_{p i}^{2}}\left(\varepsilon_{12}^{(i)} E_{b}^{*} E_{r}^{*}+\varepsilon_{11}^{(i)}\left|E_{r}\right|^{2}+\varepsilon_{22}^{(i) *}\left|E_{b}\right|^{2}+\frac{\varepsilon_{11}^{(i)} \varepsilon_{22}^{(i)}}{\varepsilon_{12}^{(j)}} E_{r} E_{i j}^{*}\right)\right\} 200 a\right)
\end{aligned}
$$

Com

$$
\begin{aligned}
& \varepsilon_{12}^{(i)}=-\varepsilon_{21}^{(i)}=i \varepsilon_{1}^{(i)} \frac{\omega_{e i}}{\omega} \\
& \varepsilon_{11}^{(i)}=\varepsilon_{1}^{(i)}\left(1-\frac{3}{4} k_{r}^{2} \rho_{i}^{2}\right) \text {; } \\
& E_{22}^{(i)}=\varepsilon_{1}^{(i)}\left(1-\frac{11}{4} k_{r}^{2} \rho_{i}^{2}\right) \text {; } \\
& \frac{E_{\tau}}{E_{b}} \approx \frac{k_{r}}{k_{b}} \\
& \frac{\partial}{\partial r^{r}}\left\langle\Pi_{\theta_{r}}\right\rangle_{\hat{i}}=-\frac{w_{s i}^{2}}{8 \pi} \rho_{i}^{2} \times
\end{aligned}
$$

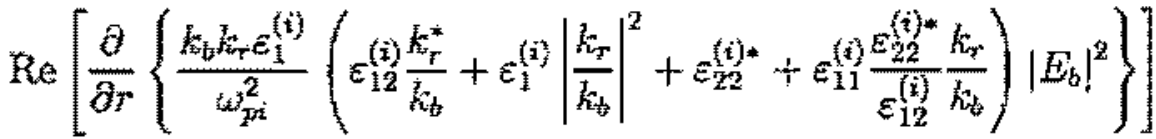

$$
\begin{aligned}
& \approx-\frac{\omega_{c i}^{2}}{8 \pi} \hat{p}_{i}^{2} \operatorname{Re}\left[\frac{\partial}{\partial r}\left\{\frac{k_{b} k_{r} E_{i}^{2(i)}}{\omega_{p i}^{2}}\left(\left|\frac{k_{r}}{k_{b}}\right|^{2}+\frac{w_{c i}}{\omega} \frac{k_{r}^{2}}{k_{b}}+1-\frac{\omega}{\omega_{c i}} \frac{k_{r}}{k_{b}}\right) \mid\left[\left.E_{b}\right|^{2}\right]\right]\right.
\end{aligned}
$$

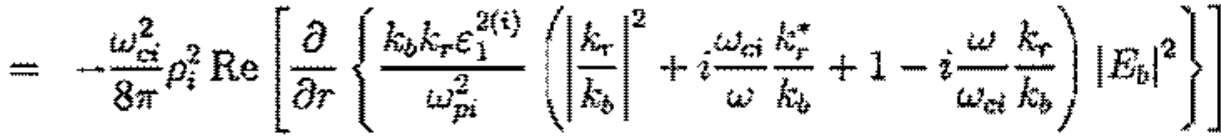

Como $\omega_{c t}>>\omega$

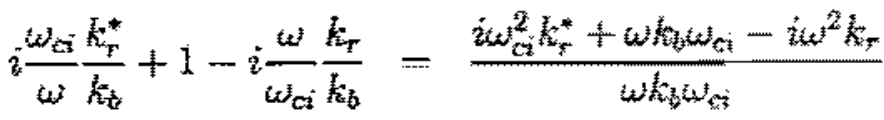

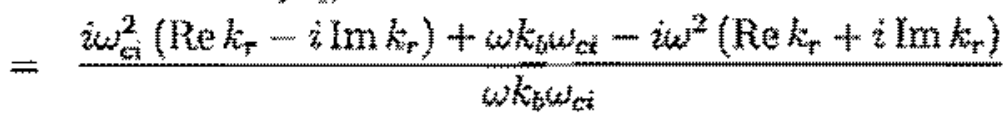

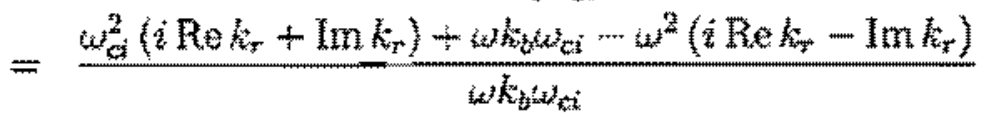

$$
\begin{aligned}
& \approx \frac{\omega_{\mathrm{a}}\left(\operatorname{Im} k_{\mathrm{r}}+i \mathrm{Re} k_{\mathrm{r}}\right)}{\omega_{k_{b}}}
\end{aligned}
$$




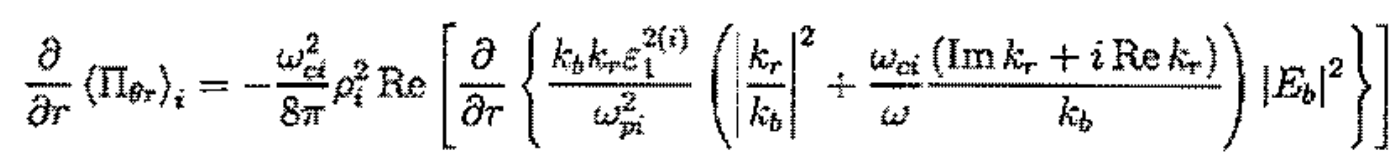

Como

$$
\begin{aligned}
& \frac{\partial}{\partial r} \approx 2 \ln \left(k_{r}\right)
\end{aligned}
$$

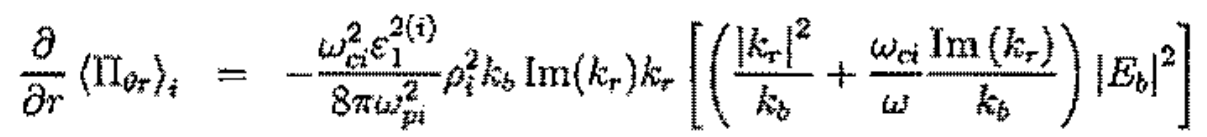

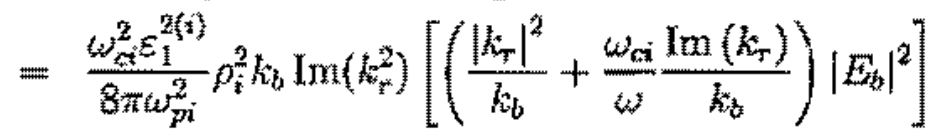

$$
\frac{k_{b}}{8 \pi} \operatorname{Im} \varepsilon_{3}\left|E_{4}\right|^{2}=\frac{k_{b}}{8 \pi} \operatorname{Im} \varepsilon_{3} \frac{\left(\frac{y^{2}}{z^{2}} \varepsilon_{1}-k_{1}^{2}\right)^{2}}{k_{11}^{4}}\left|E_{b}\right|^{2}
$$

\section{Para Ondas Magnetoacústicas Rápidas:}

Repetindo a Eq.(209a),

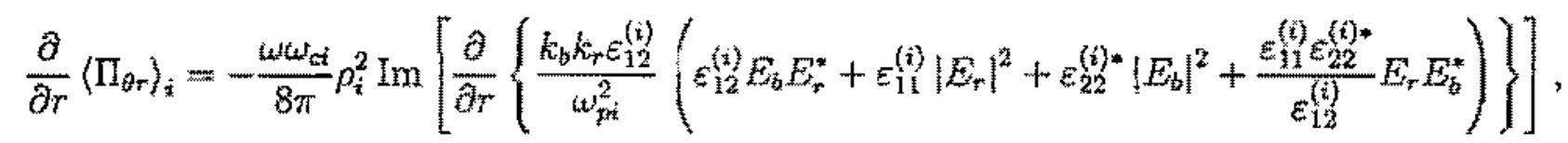

substituindo as expressões

$$
\begin{aligned}
& \varepsilon_{12}^{(j)}=-\varepsilon_{r i}^{(j)}=\varepsilon_{1}^{(j)} \frac{\omega_{c i}}{\omega} \\
& \varepsilon_{11}^{(i)}=\varepsilon_{1}^{(i)}\left(1-\frac{3}{4} k_{r}^{2} p_{i}^{2}\right) \\
& \varepsilon_{22}^{(i)}=\varepsilon_{1}^{(i)}\left(1-\frac{11}{4} k_{r}^{2} p_{i}^{2}\right) \\
& \frac{E_{r}}{E_{\alpha b}} \approx-\frac{k_{t} w_{b}+\frac{\omega^{2}}{\varepsilon^{2}} \varepsilon_{12}}{k_{r}^{2}}
\end{aligned}
$$

com

$$
\begin{aligned}
& \frac{\partial}{\partial r}\left\langle\mathrm{H}_{\partial r}\right\rangle_{i}=\frac{w_{c}^{2}}{8 \pi} \rho_{i}^{2} \times
\end{aligned}
$$

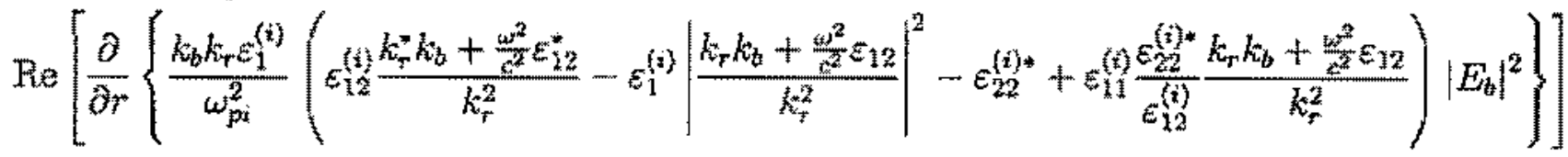




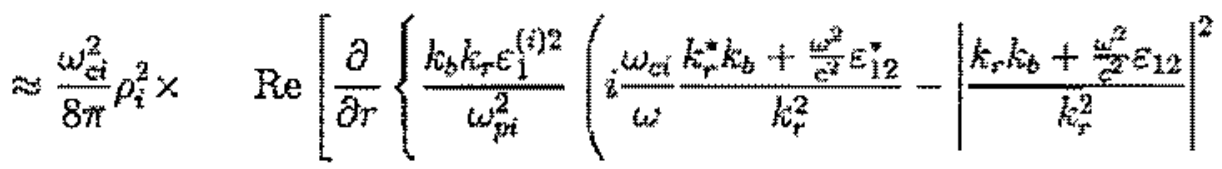

$$
\begin{aligned}
& \left.\left.\left.-\left(1-\frac{11}{4} k_{r}^{2} \rho_{i}^{2}\right)-i \frac{\omega}{\omega_{c i}}\left(1-\frac{3}{4} k_{r}^{2} \rho_{3}^{2}\right)\left(1-\frac{11}{4} k_{r}^{2} \rho_{i}^{2}\right) \frac{k_{r} k_{b}+\frac{\omega^{2}}{\alpha^{2}} \varepsilon_{12}}{k_{r}^{2}}\right)\left|E_{b}\right|^{2}\right\}\right]
\end{aligned}
$$

$$
k_{r}^{2} \approx \frac{w^{2}}{t^{2}} \varepsilon_{1}^{(j)} \quad \operatorname{Im} k_{*}<<k_{\mathrm{r}}
$$

$$
\begin{aligned}
& =\frac{\omega_{c i}^{2}}{8 \pi} \rho_{i}^{2} \times \operatorname{Re}\left[\frac { \partial } { \partial r } \left\{\frac { k _ { b } k _ { r } \varepsilon _ { 1 } ^ { ( j ) 2 } } { \omega _ { p i } ^ { 2 } } \left(i \frac{\omega_{c i}}{\omega}\left(\frac{k_{b}}{k_{r}}-i \frac{\omega_{c i}}{\omega}\right)-\left|\frac{k_{b}}{k_{r}}+i \frac{\omega_{c i}}{\omega}\right|^{2}\right.\right.\right. \\
& \left.\left.\left.-1+\frac{11}{4} k_{r}^{2} \rho_{i}^{2}-i \frac{\omega}{\omega_{c i}}\left(1-\frac{3}{4} k_{r}^{2} \rho_{i}^{2}\right)\left(1-\frac{11}{4} k_{r}^{2} \rho_{i}^{2}\right)\left(\frac{k_{s}}{k_{r}}+i \frac{\omega_{c i}^{2}}{\omega}\right)\right)\left|E_{b}\right|^{2}\right]\right]
\end{aligned}
$$

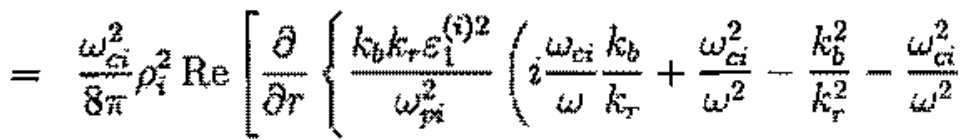

$$
\begin{aligned}
& \left.\left.\left.-1+\frac{11}{4} k_{r}^{2} p_{z}^{2}-i \frac{\omega}{\omega}\left(1-\frac{7}{2} k_{r}^{2} \rho_{i}^{2}+\frac{33}{16} k_{r}^{4} p_{i}^{1}\right)\left(\frac{k_{b}}{k_{r}}-i \frac{\omega_{c i}}{\omega}\right)\right)\left|E_{t}\right|^{2}\right\}\right]
\end{aligned}
$$

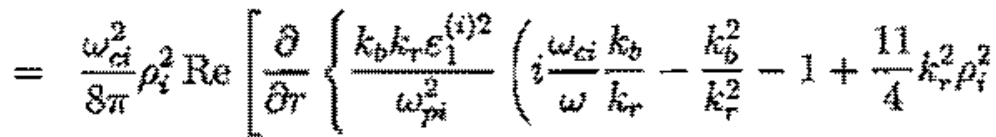

$$
\begin{aligned}
& \left.\left.\left.-i \frac{\omega}{\omega_{c i}}\left(1-\frac{7}{2} k_{r}^{2} p_{i}^{*}+\frac{33}{i 6} k_{p}^{4} p_{i}^{4}\right)\left(\frac{k_{b}}{k_{r}}+i \frac{\omega_{c i}}{\omega}\right)\right)\left|E_{i j}\right|^{2}\right]\right]
\end{aligned}
$$

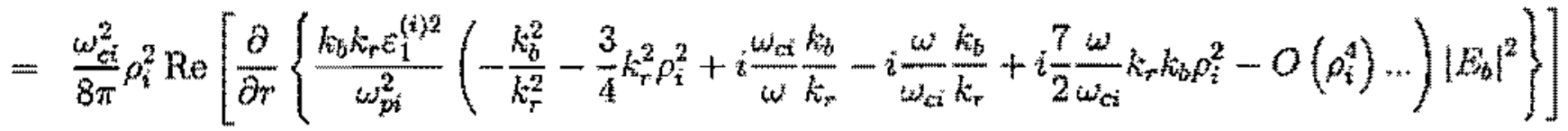

$$
\begin{aligned}
& \approx \frac{\omega_{r i}^{2}}{8 \pi} \rho_{i}^{2}\left[\frac{\partial}{\partial_{r}}\left\{\frac{k_{b} k_{r} \varepsilon_{i}^{(i) 2}}{\omega_{p i}^{2}}\left(-\frac{k_{b}^{2}}{k_{r}^{2}}-\frac{3}{4} k_{r}^{2} \rho_{i}^{2}\right)\left|E_{b}\right|^{2}\right\}\right]
\end{aligned}
$$




\section{G Efeito da rotação cisalhada nos modos Kink exter-}

\section{nos}

Os resultados deste apêndice estäo expostos nas referências [67] e [65].

Um dos problemas para a viabiliataçäo de reatores temonucleares baseados na configuraçầ de equilíbrio magnético com simetria axial, do tipo tokamak, vem sendo as instabilidade dismuptivas, que podem destruir a confguraçă de equilibrio. Estas instabilidades ocorrem como uma evoluçäo näo linear de modos de reconexảo, Tearing Modes, ou com o surgimento muito rápito, numa escala de microsegundos, de modos globais descritos pela magnetohidrodinâmica (MHD) ideal. Ha muito tem se tentado produzir configuraçöes de campo magnético que evitem tais instabilidades para tal é necessário se estudar sob quais condiçâs elas ocorrem e os fatores que podem influir positiva ou negativamente no seu controle. Neste sentido é vital lembrar os trabalhos de Bernstein et al. (1958), com o estabelecinento de um principio de energia; Newcomb (1960), que derivou condiçōes para a estabilidade de um pinch linear difuso; Hain e Lüst (1958) que, linearizando as equaçōes de movimento perturbadas, trataram o problema da estabilidade como um problema de autovalores; Goedbloed e Sakanaka (1973) que desenvolveram como alternativa an principio de energia o conceito de estabilidade $\sigma_{\text {s }}$ entre outros.

0 modelo MHD esta ntre os mais simples aplicados to estudo de plasmas magnetizatos. Neste modelo o plasma é representado como um fluido condutor perfeito, sem viscosidade, e a difusäa e a condıtçāo de calor não são incluidas. Entre as instabilidades descritas pela MHD ideal, as de maior importancia (que possuem maiores taxas de crescimento) sẫo as chamadas instabilidades kink (instabilidades de dobra helicoidal). Tal instabilidade aparece quando a coluna de plasma sofre uma perturbaçăo de maneira a ser produzida uma dobra (kink) em seu eixo, sem que sta seçäo circular seja afetada, pode-se 
facilmente visualizar que as linhas de campo no lado côncavo se aproximam e as do lado oposto se distanciam umas das outras (Fig. 4.6).
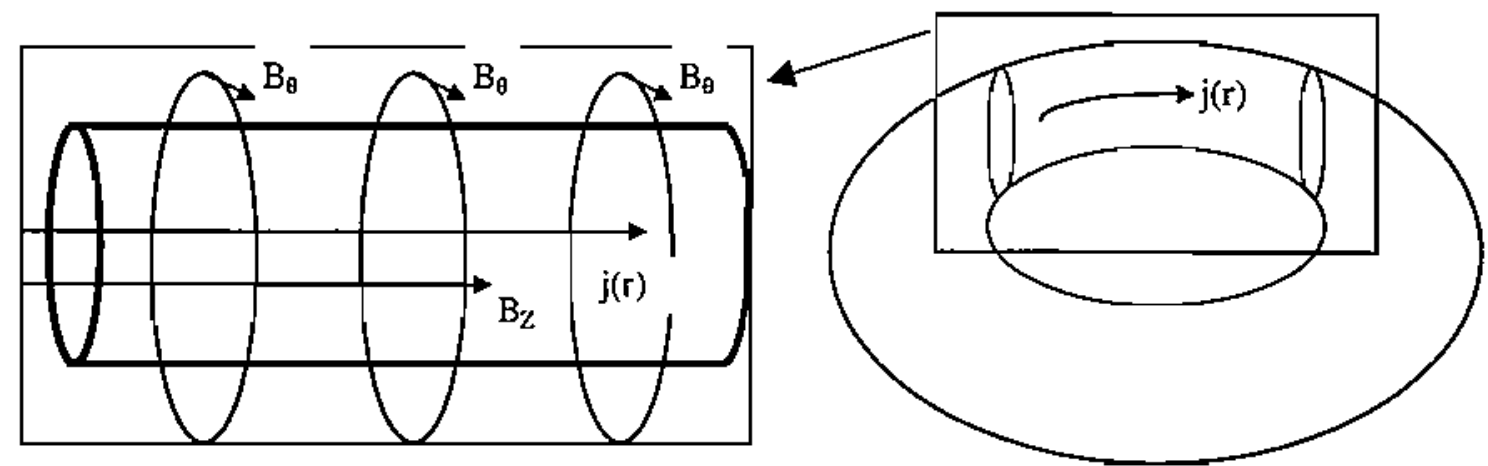

Fig. G.1 - Seçāo cilíndrica da coluna de plasma com aproximaçāo para um tokamak.

Desta forma, uma perturbação provoca um aumento da pressão magnética do lado côncavo da dobra e uma diminuiçāo no lado convexo, aumentando a perturbaçāo. O plasma é finalmente empurrado na direçāo da parede metálica que o envolve e assim destruído. Processos deste tipo sāo denominados instabilidades kink ou modos kink externos. As taxas de crescimento destes modos foram originalmente calculadas por Kruskal (1954) e Shafranov (1957). Shafranov ${ }^{[68]}$ também estabeleceu as condiçōes para as quais os modos kink seriam estáveis (demarcou os pontos de estabilidade marginal) e analizou, entre outras coisas, o efeito de estabilização da parede condutora para diferentes perfís de densidade de corrente $j(r)$.

Tradicionalmente, as instabilidades kink tem sido estudadas supondo-se o plasma em um equilibrio estático. Esta hipótese se baseia em dois fatos. Em primeiro lugar, de acordo com a teoria MHD (e neoclássica), qualquer rotação da coluna de plasma no sentido poloidal deve ser fortemente amortecida devido à viscosidade dos íons. Em segundo lugar, a taxa de crescimento dos modos kink é extremamente alta, com um tempo característico de Alfvèn, $\tau_{A}=a / V_{A}$, onde $a$ é o raio menor da coluna toroidal de plasma e $V_{A}$ é a velocidade característica de Alfvèn (ver capítulo 2). Enquanto isso, a velocidade de rotaçāo do plasma, tanto no sentido poloidal quanto toroidal (quando há 
injeção de partículas neutras), não pode vltrapassar a velocidade acústica $V_{s}$, já que esta é a velocidade com que diferenças de pressẫo se propazam. Como $V_{S} / V_{A}=\sqrt{\emptyset}<<1$ para plasmas ern tokamaks ( $\beta$ é a razano entre a pressăo cinética e a pressano do campo magnético de equilibrio), a rotação da colma de plasma seria um fenômeno demasiado lento para influir na dinâmica de fenômenos rápidos tais como os motos kink.

Nesta seção, por meío de um modelo MHD ideal incompressivel e com densidade de corrente uniforme, cálculos analíticos mostram o efeito da rotaģäo poloidal cisalhada nas taxas de crescimento dos modos kink.

Nàs Equaçōes MHD,

$$
\begin{aligned}
\rho(d \vec{m} / d t) & =-\vec{\nabla} p+\left(1 / \mu_{0}\right)(\vec{\nabla} \times \vec{B}) \times \vec{B} \\
(\partial \rho / \partial t)+\vec{\nabla} \cdot(\rho \vec{t}) & =0 \\
(d / d t)\left(p / \rho^{\gamma}\right) & =0 \\
(\partial / \partial t) \vec{B} & =\vec{\nabla} \times(\nu \times \vec{B}) \\
\vec{\nabla} \cdot \vec{B} & =0
\end{aligned}
$$

onde $p$ é a densidade do fluido, $p$ e pressäo cinétitea, $\vec{B}$ é o campo magnético, $\vec{v}$ a a velocidade de equilibrio e $\gamma=c_{p} / c_{n}$ a razão dos calores especificos, mais a equaçän do equilibrio com velocidade apenas na direçäo poloidal

$$
\frac{d}{d r}\left(p+\frac{B^{2}}{2 \mu_{0}}\right)=p \frac{v_{0}^{2}}{r^{r}}-\frac{B_{a}^{2}}{\mu_{0}{ }^{r}}
$$

é feitu a propagaçăo de uma perturbaçäo lagrangeana $\vec{\xi}$ de um elemento infinitesimal do plasma,

$$
\vec{F}=\vec{r}_{0}+\vec{\xi}\left(\vec{r}_{0}, t\right)
$$

perturbaçäo esta na qual se pode fazer uma transformaçăo de fourier

$$
\vec{\xi}(r, \theta, z)=\vec{\xi}(r) e^{i(m \theta+k z+i n t)}
$$


e obtẻm-se uma expressão perturbada para o nomento linear na forma

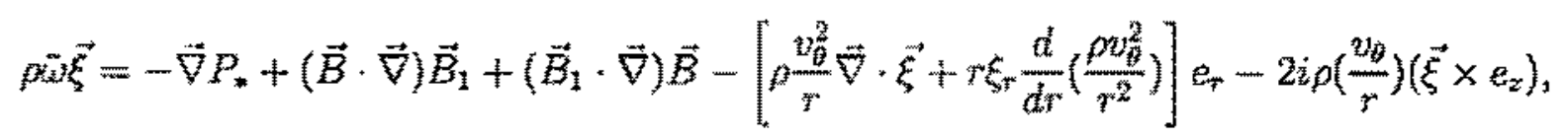

onde

$$
\begin{aligned}
& P_{*}=-\gamma p(\vec{\nabla} \cdot \vec{\xi})-(\vec{E} \cdot \vec{\nabla}) p+\vec{B} \cdot \vec{B}_{1} \\
& \vec{B}_{1}=\vec{\nabla} \times(\vec{\xi} \times \vec{B}) \\
& \vec{\omega}=\omega-m \frac{\omega}{r} .
\end{aligned}
$$

Fazendo-se a aproximaçăo para grandes comprimentos de onda, $m^{2}>k^{2} r^{2}$ no limite incompressível,

$$
\begin{aligned}
& \vec{\nabla} \cdot \vec{\xi} \rightarrow 0, \\
& \gamma p \rightarrow \infty,
\end{aligned}
$$

e impondo as condiçoses de contarno adequadas para uma configuraçāo cilíndrica periódica $(L=2 \pi R)$ com uma coluna de plasma de raio a circundada por uma camada de vácuo muma casca metalica de raio $b(\mathrm{Fig} .4 .7)$,

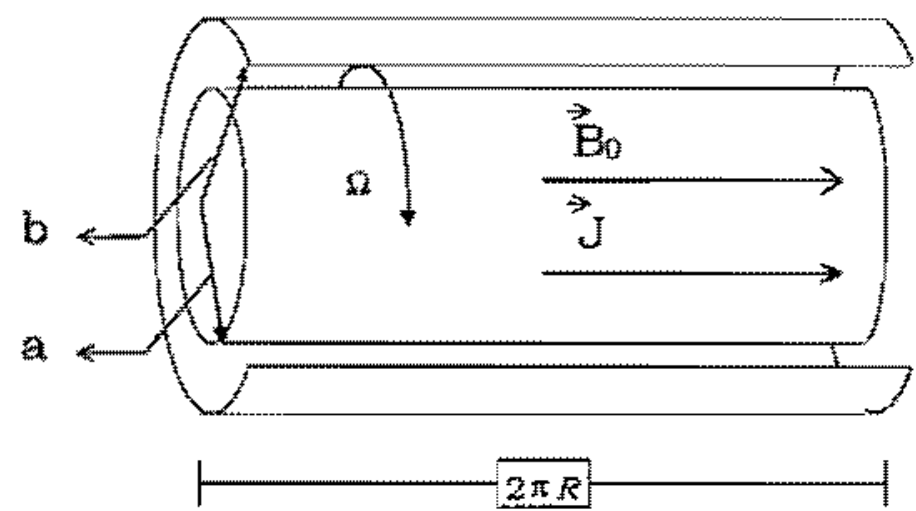

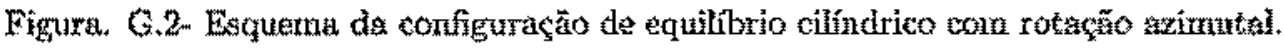

resta definir um perfil para a densidade de corrente $j(r) \hat{e}_{z}$ e para a velocidade angular de rotação $\Omega(r)=v_{\theta} / r$, a fim de se obter a soluçāo para a evoluçấo dos modos kink. 0 único caso em que se pode resolver o problema analiticamente é aquele em que a densidade de corrente e a velocidade angular sāo constantes (rotor rígido). Para estes, as 
soluçōes encontradas indicam desestabilizaça mais acentuada dos modos $m>1$, e o modo $m=1$ cego a rotaçă As taxas de crescimento, normalizadas pela velocidade de

Alfvén são dadas pela parte imagináư da frequenencia da perturbação lagrangeana

$$
\begin{gathered}
\omega=\Omega(m-1) \pm\left\{\Omega^{2}(1-m)-2 \frac{B_{a}^{2}(a)}{\mu_{0} a^{2} p}\left(m-n q(a)-\frac{(m-n q(a))^{2}}{1-\left(\frac{a}{b}\right)^{2 m}}\right)\right\}^{1 / 2} \\
\Omega_{A}^{2}(a)=\frac{V_{A}^{2}(a)}{a^{2}}=\frac{B_{0}^{2}(a)}{\mu_{0} \rho a^{2}}=1 .
\end{gathered}
$$

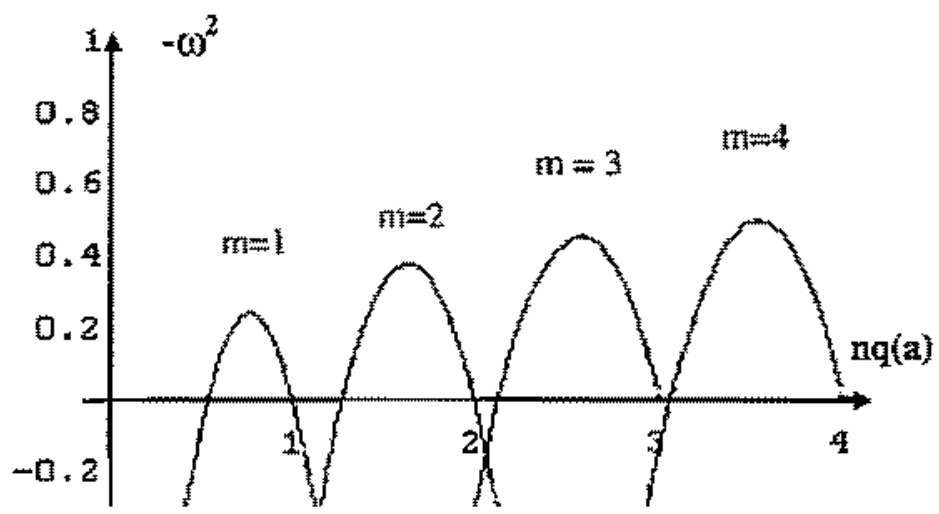

Figura G.3 - Taxas de crescínento kink em funçăo do produto do numero de onda toroidal $n$ e o fator de segurança ra bordi, $q(a)$, para os diftrentes modos polokdais no caso de densidade de cornente unitorme a sem rotação (Shatranov).

No caso em que a rotaçăo está ausente, obtêm-se os mesmos resultados de Shafranov (Fyg. 4.8) para qualquer modo poloidal $m$. Este resultado para a densidade de corrente constante mostra as taxas de crestimento maiores para $m>1$. No caso de perfis mais realisticos (com a densidade caindo até próximo de zero em $r=a$ ), o modo $m=1$ é mais importante.

Para simular a situaģäo de una rotaçăo cisalhada, deve-se introduzir uma descontinuidade no perfil da rotaçăo poloidal, para um certo $n=c_{3}$

$$
\begin{cases}\operatorname{Regiau} I, v_{0}=\Omega_{t} & \rightarrow 0<\tau<e \\ \operatorname{Regiäo} I I, u_{0}=\Omega_{I T} r & \rightarrow c<r \leq a\end{cases}
$$


Desta maneira, é necessário resolver as condições de contorno na interface destas duas camadas concêntricas de plasma, girando a velocidades diferentes, as duas quantidades que são contínuas em $r=c$ sāo o deslocamento radial,

$$
\left\|\xi_{r}\right\|_{r=c}=0
$$

e o balanço de forças, onde fica explícita a ação da rotação diferencial para uma porçāo de plasma que invade a regiāo vizinha na interface $r=c$,

$$
\left\|C_{1} r \frac{d}{d r}\left(r \xi_{r}\right)\right\|_{r=c}=0
$$

onde

$$
C_{1, \text { out } I}=\frac{F^{2}}{\mu_{0}}-\rho\left(\omega-m \Omega_{\text {Ior } H}\right)^{2}=\frac{F^{2}}{\mu_{0}}-\rho \tilde{\omega}_{\text {Ior } H}^{2}
$$

e $F=\vec{k} \cdot \vec{B}=\frac{m}{r} B_{\theta}+k B_{z}$.

Encontrando as soluçōes para $\xi_{r}$ e a pressāo total perturbada $P_{*}$ e inserindo-as nas condições de contorno para $r=a$, obtém-se as soluções através de uma equação algébrica de quarto grau, cujas soluçōes sāo simétricas com relação ao sentido da rotação e trazem informação não só da instabilidade kink, mas da instabilidade de Kelvin-Helmholtz que aparece como conseqüência da descontimuidade da rotação. As figuras que seguem ilustram a evolução das taxas de crescimento a partir da situaçāo de Shafranov $\left(v_{\theta}=0\right)$.

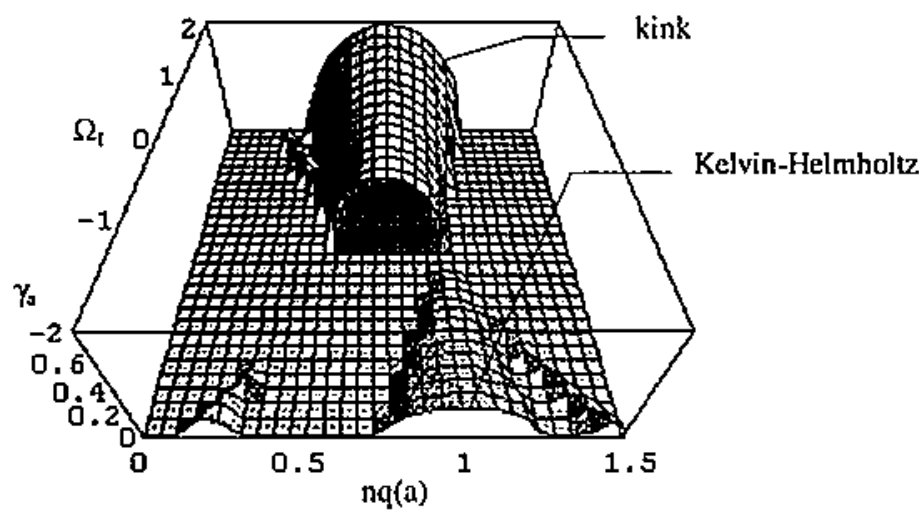

Figura G.4a - Forma geral de uma das soluções em que a parte imaginária positiva de $\omega$ (taxa de crescimento da instabilidade) é mostrada como função da velocidade de rotação $\Omega_{I}\left(\Omega_{I I}=h \Omega_{I}\right)$ e do 
produto do número de onda toródal $n$ e o fator de segurança na borda do plasma $q(a)$. Neste gráfico, $h=0,75$ e $c / a=0,5$
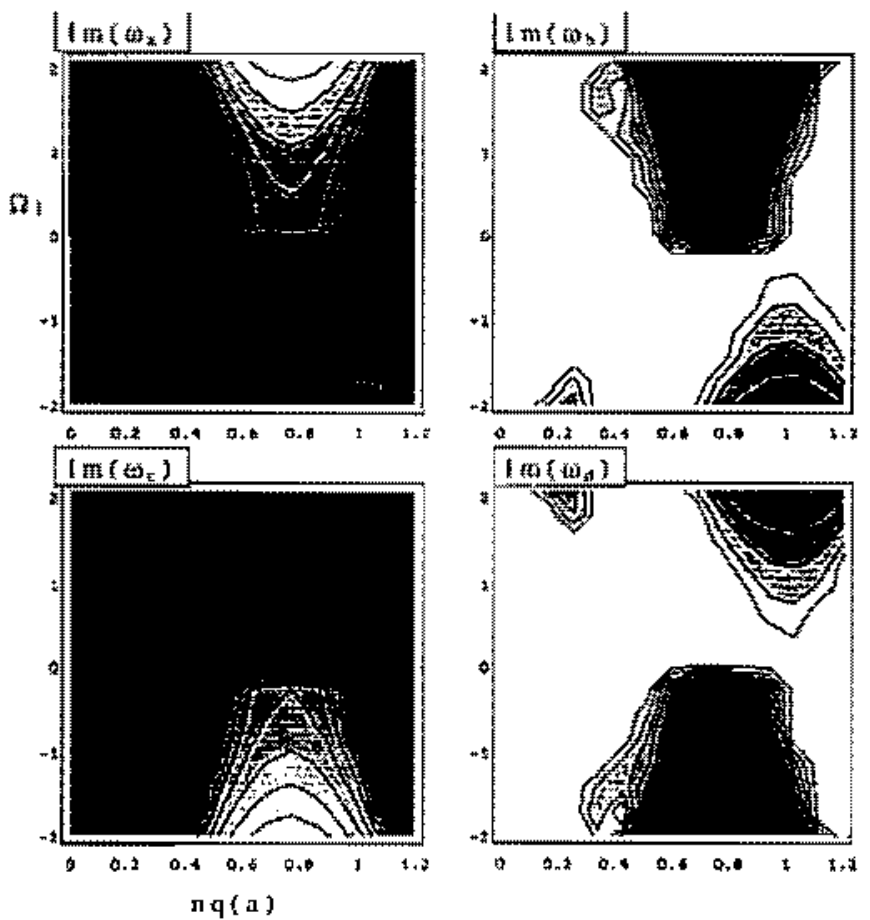

Figura G.4b- Exernplo da forma das solucoes para $\gamma=\ln \omega$. Nestas curvas de nivel as soluçôes

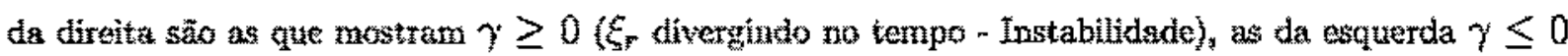

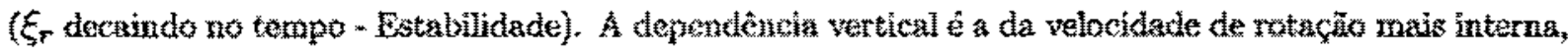
$\Omega_{r}$, normalizada para a velocidade de Alfvén (obviamente aqui com valores exagetatios), onde se vê que

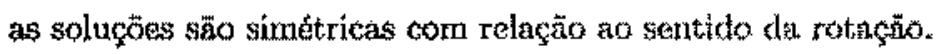

A soluçăo para o caso em que o cisalhamento se dá próximo à borda do plasma, com a rotagäo externa maior que a intema (como aconteceria no caso de uma rotagäo fortemente cisaluada na periferia do plasma) e com os parametros do TOABR, é mostrada na Fig. 4.10, demonstra, para uma rotaçăno com valor inferior a $5 \%$ da velocidade de Alfuén (neste caso um valor subsônico) a completa estabilização do modo kink, enquanto a taxá de crescimento de Kelvin-Helmholtz é, comparativamente bem pequena. 


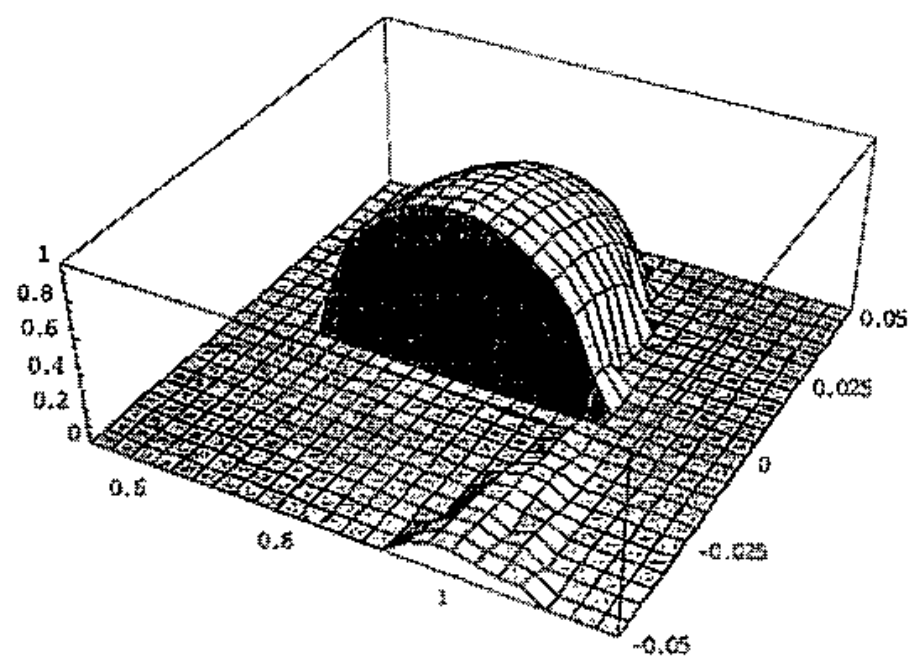

Figura C..5a - Completa Estabilizaçāo do modo kink para um cisallumento da velocidade polódal

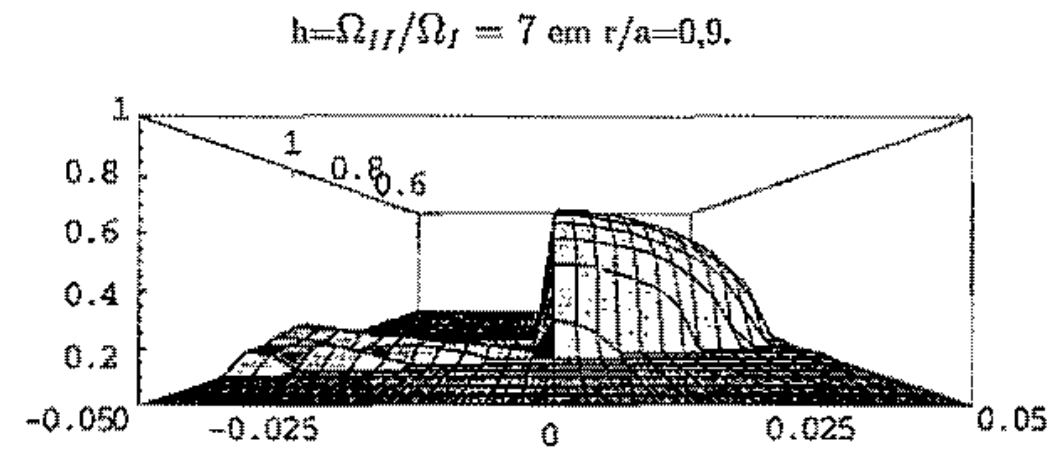

Fügura $6.5 b$ - Mesmo restltado de $4.10 \mathrm{a}$ visto de outro ângulo.

É necessário ainda se refazer estes cálculos para perfís mais realisticos de densidacte de corrente e de rotação. Para tal, um código numuerico mass completo é necessário. Os resultados expostos neste trabalbo analítico servem, todavia, como indicativo do comportamento do sistema em resposta à rotação cisalhada. Esta resposta estabilizadora é devida à força de Coriolis que, provocada pela rotação maior da camada mais externa, dificulta a deformação da coluna de plasma. 


\section{Referências}

[1] F. F. Chen, Introduction to Plasma Physics, PlenumPress, New York (1974).

[2] J. A, Bittencourt, Fundamentals of Plasma Physics, Pergamon Press, Oxford (1986).

[9] Bigliari, P. H. Diamond and P. W. Terry; Phys. Fiztids B 2, 1 (1990).

[4] J. Groebner, K. H. Burrel and R. P. Seraydarram; Phys. Rev. Letw. 64, 3015 (1990).

(5) C. Shaing and E. C. Crume Jr; Phys. Rev. Lett. 63, 2369 (1089).

[6] K. H. Burrell, Phys. Plasmas 4, 1499 (1997).

[7] H. Alfvén: Nature, 160, 405 (1942).

[8] A. G. Elinmov, A. G. Kirov, V. P. Sidorov High Frequency Plasma Heating, edited by A. G. Litvak (Translation Series, American Institute of Physics, New York) p. 239 (1992)

[9] G. A. Collins, F. Hofmann, B. Joye et ah,: Phys. Fhuids, 29, 2260 (1986).

[10] R. Majeski, et ol, in the Procedings of the $4^{\text {th }}$ International Conference on Plasma Physics e Controlled Nuclear Fusion Research, Wurburg 1992 (IAEA, Vienna, 1993), v. 1, p. 751 .

[11] J. Vaclavic, K. Appert, Nuclear Fusion, 31, 1945 (1991).

[12] E. Priest,: Solar Magnetohydrodynamics, D. Reidel Publ. Co., Dordrecht, Holland (1982).

[13] V.V. Braginskii,; Rev. Plasma Phys, edited by V.S. Leontovich, (New York, Consultants Bureau, 1965) 1, 205.

[14] F. M. Nekrasov, Sov. Journ.Plasma Phys., 18, 520 (1992). 
[15] M. V. Dmitrieva, S. Yu. Medvedev, G. A. Pestryakova, A. G. Elfimov, K. G. Ko mosbvili, and V. P. Siderov, Proceddings of the 16th EPS Conference on Controlled Fusion and Plasma Physics, Venice, edited by S. Segre, H. Knoepfel, and E. Sindone (European Physical Society, 1989), v. III, p. 423.

[16] V. P. Sidorov, F. M. Nekrasov, K. G. Konoshvili, A. G. Elfimov, A. P. Favorskij, V. F. Tishkin, and M. V. Dmitrieva, Nucl. Fugion 27, 1411 (1987).

[17] A. G. Elfimov, A. G. Kirov, V. P. Sidorov, In High Frenuency of Plasma Heating, edited by A. G. Litvak, (Transh. Series, AIP, New York, 1992) p. 239.

[1.8] A. G. Kirov, V. P. Sidorov, A. G. Elfimov, et al., In the Proceedings of the $1.3^{\text {th }}$ International Conference on Plasma Physics e Controlled Nuclear Fusion Research, Kioto, 1986 (International Atomic Energy Agency, Viemna, 1987), Vol. 1, p. 645.

[19] A.G. Elfimov, J.A.Tataronis, N. Hershkowity, Physics of Plasmas, 1, 2637 (1994).

[20] D. W. Ross, G. L. Chen, S. M. Mahajan, Phys, Fuids, 25(4), 652 (1982).

[21] D.Grekov, K.N.Stepanov, J.Tataronis, Sov. J. Plasma Phys. v.7,752 (1981).

[22] P.M. Bellan, Physics of Plasmas 1, 3523 (1994).

[23] M. Abramowitz, I.A. Stegun: Hebook of Mathematical Fundtios, Dover Publicação Inc., New York (1972) p.555-587.

[21] Akira Hasegawa e Chanchal Uberoi, The Alfǚn wave (Technical Informação Center U.S. DOE, 1982].

[25] L.N. Nosova, S.A. Tumarkin: Tables of generalized Airy funç̧ôs for the asymptotic solução of the differential equaçōes (Pergamon Press., Oxford, 1965) p.p. IX-XV. 
[26] L. Chen e A. Hasegawa, Physics of Fluids 17(7), 1399-1403 (1974).

[27] K. Appert et al, Plasma phys. Contr. Fusion 28, 133 (1986).

[28] W. Q. Li , D. W. Ross and S. M. Mahajan Phys. Fituids B 12353 (1.989).

[29] K. Appert, J. Vaclavik and L. Villard, Phys. Fhuids 27, 432 (1984).

[30] A.G. Elfimov, M.V. Dmitrieva, A.A. Twanov, et al. - In: Proced. of 9th European Confer. CONTROLLED FUSION AND PLASMA PHYSICS. Dubrovnik, May (1988), part III, p.944. A.G. Elfimov - ibid, p.964.

[31] A. Becoulet Plasmat Plys. Conir. Fusion 38, A.1-A11 (1996).

[32] W. Q. Li Kinetic Effects in Alfyén and lon Cyclotron Wave Propagation: Surface Eigenmodes and Impurity Effects. Tese de doutoramento defendida no Fusion Research Center, Universidade do Texas em Austin. (1988)

[39] L. Rucliko, M.C.R. Andrade, et al. In Proceedings of the $10^{\text {th }}$ International Conference on Plasma Physics, Foz do Iguaçu, November 1994, Brazil, 1, p.365.

[34] A.G. Elfimov, C. A. de Azevedo, A. S. de Assis Solar Phys. 167, 203 (1996).

[35] A.G. Elfimov, R. M. O. Galväo, 1.C. Nascimento and G. Amarante-Segundo Plasma Phys. Contr. Fution $3915 \tilde{s} 1$ (1997).

[36] R.M.O. Galväo, A.G. Elfimov, G. Amarante-Segundo, V. S. Tsypin, L..F. Ruchko, I. C. Nascimento and M. Tendler, Plasma Phys. Contr. Fission 41 A487 (1999).

[37] Kolesnichenko Ya 1, Parail V V, Pereverzey G V Reu. Plasma Phys. edited by Kadomtsev B.B. (Consultants Bureau, New York, 1990) 17, 3-155.

[38] Synakowski E J, Batha S H, Beer M A, et al. Phys. Rev. Letters, 78, 2975 (1997). 
[39] G. M. Staebler, R. E. Waltz, J. C. Wiley Nuclear Futsion, 37, 287 (1997).

[40] N. J. Fisch, and C. F. F. Karney Phys. Fuids, 24, 27 (1981).

[41] D.A. Ehst, and C. F. F. Karney Nucl. Fusion 31, (1933).

[42] A. G. Elfimov and Puri S. Nuclear Fusion, 30, 1215 (1990).

[43] Ohkawa T, Comments Plasme Plys. Controlled Fusion, 12, 165 (1989).

[44] J. B. Taylor, Phys. Rev. Lett., 63, 1384. (1989).

[45] A. G. Elfimov, Sov. J. Plasma Phys., 17 (3), 223 (1991).

[46] R. Klíma, Czech. J. Phys. B30, 874 (1980).

[47] V. S. Tsypin, A. G. Elfimoy, C. A. de Azevedo, A. S. de Assis, Physical Review E, $51,2662(1995)$.

[48] V. S. Tsypin, A. G. Elfimov, C. A. de Azevedo, A. S. de Assis, Phys. Plasmas, 3, $4606(1996)$.

[49] Mikhailowskii A.B., and Tsypin V.S. Sov. J. Plasma Phys. 10, 142 (1984).

[50] S. P. Hirshman, D. J. Sigmar Nuclear Fusion,10, 1079 (1981).

[51] A.G.Elfimov, R.M.O.Galväo, I.C. Nascimento, and G. S.Amarante-Segundo Plasma Phys. Contr. Fusion 40, 451 (1998).

[52] G. S. Amarante-Segundo, A.G. Elfirnov, D.W. Ross, R.M.O. Galvăo Phys. Plasmas, $6,2437(1999)$.

[53] T. S. Hahm and K. H. Burrel, Phys. Plasmas 2, 1648 (1995). 
[54] V. S. Tsypin, R. M. O. Galvão, I. C. Nascimento, A. G. Elfimov, M. Tendler, C. A. de Azevedo, and A. S. de Assis, Phys. Rev. Lett. 81, 3403 (1998).

[55] K. O. Shaing and M. C. Zarnstorf, Phys. Plasmas 4, 3928 (1997).

[56] K. C. Shaing, A. Y. Aydemir, Y.R. Lin-Liu, and R. L. Miller, Phys. Reu. Letw 79, $3652(1997)$.

[57] V. S. Tsypin, A. G. Elfimov, M. Tendler, A. S. de Assis, and C. A. de Azevedo, Phys. Plasmas. 5, 7 (1998).

[58] H. L. Berk and A. A. Galeev, Phys. Fluids 10,44 (1967).

[59] K. C. Shaing and R. D. Hazeltine, Phys. Fluids B, 4, 2547 (1992),

[60] K. C. Shaing, R. D. Hazeltine, and M. C. Zarnstorff, Phys. Plasmas 4, 1371 (1997).

[61] A. G. Elfimov, Comments Plasma Phys. Controll. Fusion 17, 145 (1996).

[62] A.G. Elfmov, V. Petratlex, and J. A. Tataronis, Physics of Plasmos, 1, 2882 (1994).

[63] A.G. Elimov In: Proc. of 2nd International Symposium HEATING IN TOROIDAL PLASMA. Como-Italy. September, v.2, p.683 (1980).

[64] G.G. Craddock and P.H. Diamond, Plys. Rov. Lett. 671535 (1991).

[65] G. S. Amarante Segundo-Dfeito do Rotacta Csalhada nos Modos Kint Eutemose Aparecimento dos Modos de Keltun-Helnholtz - Dissertacão de mestrado defendida no Instituto de Física da USP (1996).

[6b] E. Butkov, Fiston Matemátża, Editora Guanabara, Rio de Janeiro (1988).

[67] G. S. Amarante Segundo and R. M. O. Galvăo, Commenta pasma phys. Controlled Fusion 18, n 5, p. 335 (1998). 
[68] V. D. Shafranov, Sov. Phus. - Tech. Phys. 15, 175 (1970).

[69] G. Besson, G. A. Collins, P.B.Dutual, et al, In the Proceedings of the $13^{\text {th }}$ Internaçäoal Conference on Plasma Physics e Controlled Nuclear Fusion Research, Kioto, 1986 (International Atomic Energy Agency, Vienma, 1987), vol. 1, p. 617.

[70] N.F. Cramerand C-M. Yung Plasma Puss. Contr. Fusion 28, 1043 (1986).

[71] R. A. Demirkhanov, A. G. Kirov, et al., Sovet Phys. JETP Letters, 3328 (1981).

[72] V.V. Dolgopolov, K.N. Stepanov, Nuclear Fusion 5, 276 (1965).

[73] B. P. Duwal, Joye B, Marchal B Nucl. Fusion, 321405 (1992).

[74] A.G. Elfmov -In: Sov.J.Plasma Phys, 10, p.700 (1984).

[75] A.G. Elfimov el al, Brazilian Jowm. Phys, 25, 224 (1995).

[76] J.P. Goedbloed, A. Lifshitz, Phystes of Plasmas 2,3550 (1095).

[77] R. Majeski, P. Probert, N. Hershkowitz, et al. In the Proceedings of the $14^{\text {th }}$ In ternational Conference on Plasma Physics e Controlled Nuclear Fusion Research, (IAEA-CN-60/A3/5-P-10) Wurzburg, Germany, (1992) (Internaçãoal Atomic Energy Agency, Vienna, 1993), Vol. 1, p. 751.

[78] Intrator $\mathrm{T}$ et al Physics of Plasmas, 22263 (1905).

[79] A. G. Krov, A. G. Elfimov, et al, In the Proceedings of the $13^{\text {th }}$ International Conference on Plasma Physics e Controlled Nuclear Fusion Research, Washington, 1990 (International Atomic Energy Agency, Viena, 1991), 
[80] R. Majeski, N.J. Fish, et al., In the Proceedings of the $15^{\text {th }}$ International Conference on Plasma Physics e Controlled Nuclear Fusion Research, (IAEA-CN-60/A-3-1-4) Seville, Spain, September 1904.

[81] M.S. Ruderman, M. Goossens, I. Zheliaskov, Puysics of Plasmas 2, 3547 (1995).

[82] L. Ruchko, E. Ozono, R.M. O. Galvão, I. C. Nascimento, F. T. Degasperi and E. Lerche, Fusion Engincening and Design 43,15-28 (1998).

[83] V. D. Shafranov Rev. Plasma Phys, edited by Leontovich, V.S. (New York, Consutants Bureau, 1964) 3, 3 .

[84] J. Tataronis e W. Grossman, Z. Plys, 261, 203 (1973).

[85] V. S. Tsypin, I. C. Nascimento, R. M. O. Galväo, A. G. Elfimov, G. S, Amarante Segundo and M. Tendler, Phys. Plasmas, 6, 3548 (1999).

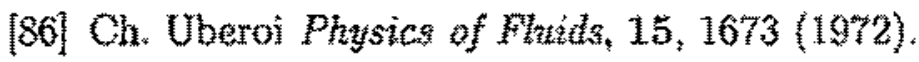

[87] S. Wukitch, M. Vukowic, at al. - Phys. Rew. Letters, 74, p.2240. Vol. 1, p. 831 (1995).

[88] S. Wukitch, C. Litwin, M. Harper, R. Parker: and N. Herclkovitz, Phys, Rev. Letters $77294(1096)$ 
